\title{
A blind man's bluff: choice blindness in eyewitness testimony
}

Citation for published version (APA):

Sagana, A. (2015). A blind man's bluff: choice blindness in eyewitness testimony. [Doctoral Thesis, Maastricht University]. Maastricht University. https://doi.org/10.26481/dis.20150917as

Document status and date:

Published: 01/01/2015

DOI:

10.26481/dis.20150917as

Document Version:

Publisher's PDF, also known as Version of record

\section{Please check the document version of this publication:}

- A submitted manuscript is the version of the article upon submission and before peer-review. There can be important differences between the submitted version and the official published version of record.

People interested in the research are advised to contact the author for the final version of the publication, or visit the DOI to the publisher's website.

- The final author version and the galley proof are versions of the publication after peer review.

- The final published version features the final layout of the paper including the volume, issue and page numbers.

Link to publication

\footnotetext{
General rights rights.

- You may freely distribute the URL identifying the publication in the public portal. please follow below link for the End User Agreement:

www.umlib.nl/taverne-license

Take down policy

If you believe that this document breaches copyright please contact us at:

repository@maastrichtuniversity.nl

providing details and we will investigate your claim.
}

Copyright and moral rights for the publications made accessible in the public portal are retained by the authors and/or other copyright owners and it is a condition of accessing publications that users recognise and abide by the legal requirements associated with these

- Users may download and print one copy of any publication from the public portal for the purpose of private study or research.

- You may not further distribute the material or use it for any profit-making activity or commercial gain

If the publication is distributed under the terms of Article $25 \mathrm{fa}$ of the Dutch Copyright Act, indicated by the "Taverne" license above, 



\section{A blind man's bluff: \\ Choice blindness in eyewitness testimony}

Sagana Anna 
ISBN: 978-94-6299-169-9

Cover image: "Tweed and Erie Rings Playing Blindman's Bluff"

Copyright (C) 2004-2015 Florida Center for Instructional Technology

Layout: Ridderprint BV - www.ridderprint.nl

Printed by: Ridderprint BV - www.ridderprint.nl 


\title{
A BLIND MAN'S BLUFF: \\ CHOICE BLINDNESS IN EYEWITNESS TESTIMONY
}

\author{
DISSERTATION \\ to obtain the degree of Doctor at Maastricht University, \\ on the authority of the Rector Magnificus, Prof. dr. L.L.G. Soete, \\ in accordance with the decision of the Board of Deans, \\ to be defended in public on \\ Thursday, $17^{\text {th }}$ September 2015 , at 16.00 hours \\ by
}

Anna Sagana 
Supervisor

Prof. dr. H.L.G.J. Merckelbach

Co-supervisor

Dr. M. Sauerland

Assessment Committee

Prof. dr. R. Ruiter (chair)

Prof. dr. E. Erdfelder (Universität Mannheim, Germany)

Prof. dr. J. Kerstholt (Universiteit Twente, The Netherlands)

Dr. K. van Oorsouw

Dr. T. Smeets 


\section{CONTENTS}

Chapter 1 General Introduction

Part $1 \quad$ Establishing the effect

Chapter 2 Theoretical and legal issues

related to choice blindness for voices

Chapter $3 \quad$ "This is the person you selected.": Eyewitnesses' blindness

for their own facial recognition decisions

Part 2

Testing blindness in the field

Chapter 4 Witnesses' blindness for their own facial recognition decisions: A field study

Chapter 5 The effect of choice reversals on blindness for identification decisions

\section{Part $3 \quad$ Expanding the application}

Chapter 6 Eyewitnesses' blindness for own- and other-race identification decisions

Chapter 7 Witnesses' failure to detect covert manipulations in their written statements

Part $4 \quad$ Unravelling the mechanism

Chapter 8 Self-relevance does not moderate choice blindness in adolescents and children

Chapter 9 Memory impairment is not sufficient for choice blindness to occur

Chapter 10 General Discussion

Summary

$\Sigma u ́ v o \psi \eta$

References

Valorisation

Acknowledgements

Publications 


\section{PREFACE}

$\Sigma \alpha v \vee \alpha \delta\llcorner\alpha \dot{\lambda \varepsilon \xi \varepsilon \varsigma}$

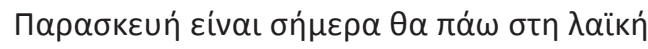

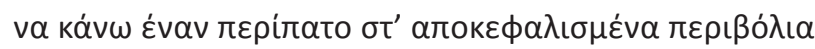

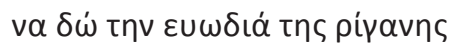

$\sigma \kappa \lambda \dot{\alpha} \beta \alpha \sigma \varepsilon \mu \alpha \tau \sigma \alpha \dot{k}\llcorner\alpha$.

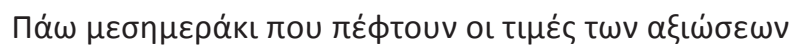

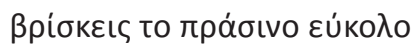

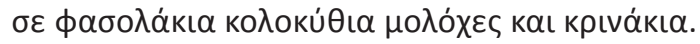

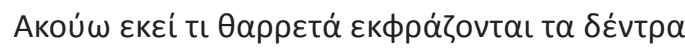

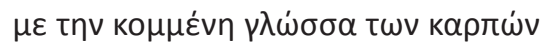

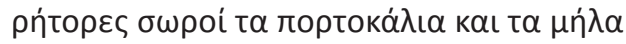

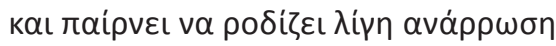

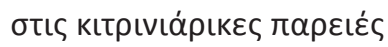

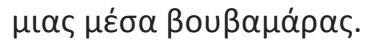

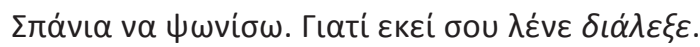

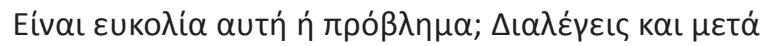

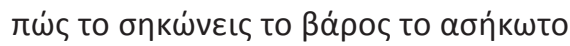

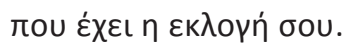

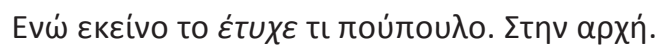

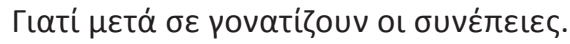

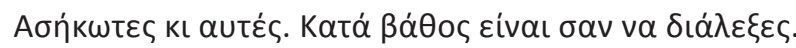

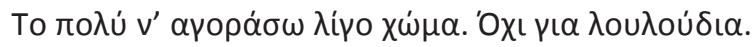

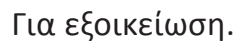

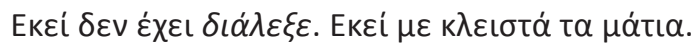

Kıкń $\Delta \eta \mu \mathrm{Ou} \lambda \dot{\alpha}$

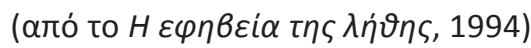


As If You'd Chosen

It's Friday today I 'm going to the market

to take a walk in the decapitated gardens

to see the oregano's fragrance

a slave in bundles.

I go in the afternoon when demands' prices have fallen

you find the green easy

on beans marrows mallows and lilies.

It's there I hear how boldly the trees express themselves

in the fruit's sharp tongue

heaps of orators the oranges and apples

and a slight recovery starts to grow rosy

in the sallow cheeks

of a muteness within.

I rarely buy. For there it's choose for yourself.

Is that an advantage or problem? You choose and then

how do you bear the unbearable burden

of your choice.

Whereas that by chance is so feather-bed soft. At first.

For afterwards you stagger beneath the consequences.

These too being unbearable. In fact it's as if you'd chosen.

If anything I'll buy a little soil. Not for flowers.

For getting accustomed.

There it's not choose for yourself. There the eyes are closed.

Kiki Dimoula

(from Lethe's Adolescence, 1994) 



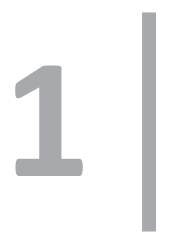

\section{General Introduction}

This chapter is an adapted and extended version of the following articles:

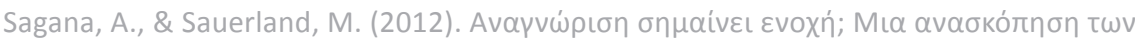

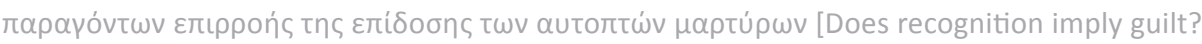
An overview of the factors that influence eyewitness identification performance]. $\Psi_{\text {uxo }}$ oyía [Psychology], 19, 63-79.

Sagana, A., Sauerland, M., \& Merckelbach, H. (2012). It's your choice! - Or is it really? The Inquisitive Mind, 14/2012. Retrieved from in-mind.org/article/its-your-choice-or-is-it-really 



\section{INTRODUCTION}

In the 1936 movie "Modern Times", Charlie Chaplin struggles to live in the fast-paced modern society. As we move from the industrial age to the information age, societal demands on our mental capabilities are far more taxing. We are constantly required to process a wide range of information to make decisions. Sometimes, these decisions are trivial, such as what marmalade to buy. At other times, the stakes are higher, such as deciding which symptoms to report to the doctor. However, the fact that we are required to processing large amounts of information does not mean that we are good at it (Chabris \& Simons, 2010). Our sensory and cognitive systems have systematic ways of failing, of which we are often (perhaps blissfully) unaware. One such fallacy of human cognition is choice blindness. In a first demonstration of choice blindness, Johansson, Hall Sikström, and Olsson (2005) asked participants to select the more attractive alternative for each of 15 different pairs of female faces. After making a decision, participants were presented with the chosen alternative and were asked to explain their judgement. However, for three of these pairs the researchers, using a magic card trick, swapped the chosen photo with the alternative non-preferred face. Thus, participants had to justify a decision they never made. Surprisingly, the overwhelming majority of participants (87\%) failed to detect the manipulations. Thus, choice blindness refers to the difficulty people have in detecting manipulations in their own previously made choices. Considering that people can be unaware of changes in the outcome of their decisions we wondered: could this be true for eyewitnesses' decisions?

It is common knowledge in cognitive psychology that the identification of nonfamiliar faces is a difficult task for most people (Johnston \& Edmonds, 2009). Yet, the judicial system relies considerably on that error prone task. In fact, eyewitness identification is considered one of the most powerful forms of evidence when deciding the conviction or exoneration of a suspect, even in the absence of further evidence. For example, in the US, the jury instructions frequently imply that the testimony of one witness is sufficient to prove a fact, if that witness is considered credible. Illustrative of this matter are the instructions given to the jury in the state of Connecticut:

\footnotetext{
"The weight of the evidence presented by each side does not depend on the number of witnesses. It is the quality of the evidence, not the quantity of the evidence, that you must consider." (Section 2.4.2, Connecticut Jury Instructions
} - Criminal, 2012).

Similar regulations can be found in the Greek Code of Criminal Procedure (CCP). There, in principle, at least two witnesses are required for the full proof of guilt of a suspect. 
Yet, based upon a so called "moral" judgment, the Code allows judges to reach a verdict from a single witness, if they consider him/her to be "highly reliable" (Sect. 177, CCP). At first glance, this does not seem to be a problem in the Netherlands. The Dutch Code of Criminal Procedure clearly states that information based on a single witness is not admissible evidence in court (unus testis nullus testis principle; sect. 338 (CP). However, if a witness statement is combined with a police record, then the unus testis nullus testis principle is no longer violated. To make matters worse, in 1926 the Dutch Supreme Court ruled that hearsay (testimonium de auditu) is admissible in court. As such a police officer's testimony about a statement provided by an actual witness during investigation can be used as evidence, without the witness ever appearing in court (Tak, 2008). Hence, often a single witness combined with another piece of evidence might be sufficient to condemn a suspect. However, even the most well-meaning witnesses and police officers can make mistakes or, as choice blindness implies, could be oblivious to alterations in their very decisions.

In this dissertation, we argue that choice blindness may be a menace to the judicial system that has received little - if any - attention from researchers. We maintain that choice blindness have a potentially important role in accounting for erroneous eyewitness testimony, both with reference to identification decisions and witness reports. Furthermore, we suggest that choice blindness may side with variables (e.g., cross-race bias, investigator bias) that are already known to undermine testimonial accuracy, resulting in a complex interplay of cognitive limitations and social influences. Therefore, the introduction of this dissertation is devoted in bridging the gap between eyewitness testimony and choice blindness. First, we present an overview of two of the most influential theories aiming to explain eyewitness decision making. This is done because choice blindness speaks to the ability of individuals to introspect over past decisions and, by extension, to eyewitness decision making. Consequently, we provide an overview of factors that could affect decision making and hence testimonial accuracy. However, we limit this overview to those variables that might interact with, influence, or be influenced by choice blindness. Subsequently, we describe the main findings of the choice blindness literature and aim to identify candidate theories that could explain the effect. Finally, drawing from the choice blindness literature, we elaborate on how blindness can arise in the legal domain, on how blindness can interact with other forensically relevant factors and impact testimonial accuracy.

\section{Eyewitness Decision Making Strategies}

The single most influential theory to explain eyewitnesses' identification decision making is the relative vs. absolute judgment strategy theory of G. Wells (1984). According to Wells, a witness using a relative judgment makes a positive identification if the match of a specific lineup member and the witness's memory is comparatively 
better than the match of any other lineup member. This elimination strategy leads the eyewitness to always choose someone from a lineup, even in the absence of the perpetrator. Conversely, a witness using an absolute judgment evaluates each lineup member against an absolute decision criterion. If the match between the individual lineup members and the witness's memory of the perpetrator is sufficiently high above the decision criterion, the witness would make a positive identification. If however, no lineup member yields a strong enough match to the memory of the perpetrator to exceed the decision criterion, no identification is made.

Though well-respected and influential, the relative vs. absolute judgment theory has not escaped criticism with the latest being that it is simply a theory about response bias (i.e., the tendency to choose; Wixted \& Mickes, 2014). Indeed, an absolute standard is nothing more than a decision criterion that can vary from liberal to conservative. Above that, the initial theory fails to incorporate factors beyond the actual memory strength that bears on the criterion setting. On that account, Brewer, Weber, and Semmler (2007) proposed that witnesses tend to assess their ability to make a positive identification not only in terms of memory strength but also in terms of metacognitions emerging during encoding and retrieval, memory heuristics and beliefs about memory.

Relatedly, Koriat, and Goldsmith's (1996) monitoring and control framework suggests that when reporting information from memory, people invoke monitoring and control processes to obstruct potentially wrong information. Thus, rather than a pure memory-retrieval mechanism, their model includes a) a monitoring process that assesses the correctness of the potential response and b) a control mechanism that determines whether to volunteer the best candidate answer through the regulation of precision and coarseness of the information (Goldsmith, Koriat, \& Weinberg-Eliezer, 2002; Koriat \& Goldsmith, 1996). That is, witnesses first retrieve a very informative and detailed candidate answer from memory. Then, the individual assesses his/her feeling of rightness (i.e., confidence in the likely accuracy) of the candidate answer and finally tests it against a criterion value. If confidence exceeds the criterion, the detailed answer is volunteered; otherwise, a less detailed and relatively coarse answer is retrieved from memory, and the monitoring and control processes are repeated. If the retrieved information is highly coarse no answer is volunteered. Thus, Koriat and Goldsmith speak in favour of an accuracy-informativeness trade-off where the feeling of rightness (likely accuracy) and informativeness are under the control of the witness and the influence of metacognitive factors (Weber \& Brewer, 2008). To date, the monitoring and control framework has been suggested not only for understanding how witnesses decide to volunteer or withhold information (e.g., Higham, 2007; Kelley \& Sahakyan, 2003; Weber \& Brewer, 2008), but also as a method to exploit eyewitnesses' ability to judge whether their memory of the perpetrator is weak and 
thus refrain from making an identification decision (Perfect \& Weber, 2012; Weber \& Perfect, 2012).

To sum up, cognitive influences and more specifically the strength of the witness's memory is essential for eyewitness decision making. Additionally, metacognitive factors and beliefs about memory seem to shape decision making. Therefore, internal and external factors that do not relate directly to the witnessed event can affect eyewitness testimony. In the following, we examine how factors such as mild changes in the instructions given to witnesses (Brewer \& Wells, 2006; Steblay, 1997) or in the lineup presentation method (Steblay \& Dysart, 2011) may result in differences in decision making and therefore be detrimental to the reliability of eyewitness testimony.

\section{The Accuracy of Eyewitness Testimony}

The question of the accuracy of witness testimony has been raised since the beginning of the $20^{\text {th }}$ century through the work of Louis William Stern, Hans Gross and John Wigmore (for a review see Sporer, 2006), whose ideas were propagated in the United States by Hugo Munsterberg (1908). At the beginning of the 1970s, Elizabeth Loftus brought to the fore the issue of eyewitness testimony yet again, by using innovative and realistic experimental methods for the study of memory (for an overview of her work see Loftus, 2005). In parallel with the study of memory of events, the issue of memory of faces arose. Soon, the cognitive and social factors which affect eyewitness testimony were divided into estimator and system variables (Wells, 1978). Estimator variables are erratic factors that exert their impact during the criminal act and the retention interval between the event and the quest to recall. They are beyond the control of the judicial system and their impact can only be estimated post-hoc. On the contrary, system variables are factors under the influence of the judicial system and can support testimonial accuracy, if handled properly. Estimator variables operate primarily in the encoding and the retention phase, whereas system variables mostly operate during retrieval.

In the following, we discuss one estimator and five system variables that can affect eyewitness decision making, both in terms of reported details and eyewitness lineup identifications that are likely candidates to relate to choice blindness. These include the cross-race bias, the influence of misleading post-event information, and the importance of proper lineup construction and administration (i.e., lineup fairness, lineup instructions, investigator bias, and post-identification feedback).

\section{Cross-Race Bias}

The cross-race bias refers to the observation that people are better at identifying persons coming from their own rather than another ethnicity. Meissner and Brigham's (2001) meta-analysis on the impact of cross-race bias on identification accuracy 
reveals that the chance of a false identification is 1.6 times greater for eyewitnesses coming from a different ethnicity than eyewitnesses coming from the same ethnicity as the target. Furthermore, eyewitnesses are 1.4 times more likely to identify familiar faces when they are from the same race. Over the years, the cross-race bias has been found for different combinations of ethnicities (e.g., Caucasian vs. Asian; Caucasian vs. African) in field and laboratory studies (Wright, Boyd, \& Tredoux, 2001). The increased false positive identification rate seems to indicate a more liberal response criterion for other compared with own-race identifications (Adams Jr, Pauker, \& Weisbuch, 2010; Meissner \& Brigham, 2001).

Several theories have been proposed to explain the recognition advantage for own compared with other race faces (for a review see Sporer, 2001b; Young, Hugenberg, Bernstein, \& Sacco, 2012). Perceptual expertise theories (Michel, Corneille, \& Rossion, 2007; Rhodes, Brake, Taylor, \& Tan, 1989; Valentine, 2001) explain the effect in terms of greater ability in processing and distinguishing own compared with other race faces. Social cognitive theories (Levin, 2000; Rodin, 1987) perceive cross-race bias as a result of an individuals' tendency to think categorically (i.e., in-group vs. out-group) and to rely on the broad social group membership instead of the unique characteristics of a face. Finally, some researchers have argued that both perceptual expertise and social cognitive factors contribute to cross-race bias (Sporer, 2001b). Given that the effect seems to result from the limitations of our cognitive apparatus and/or social factors over which we have limited control, cross-race bias might partner interact with choice blindness at the expense of testimonial accuracy.

\section{Misleading Post-Event Information}

Eyewitness testimony can be impaired by misleading information encountered after observing an event (Loftus, 1979; for a review see Zaragoza, Belli, \& Payment, 2006). This phenomenon has been termed the misinformation effect and refers to the distorted memory for past events that arises after presentation of misleading information (Loftus, 2005). In a classic misinformation paradigm participants are exposed to original event information that is followed by the presentation of some inconsistent information. The misleading details can be embedded in an initial set of questions about the event (e.g., Loftus, 1975; Loftus, Miller, \& Burns, 1978) or in a post-event narrative describing the event (e.g., McCloskey \& Zaragoza, 1985a). The typical finding is that testimonial accuracy is significantly lowered when misinformation is presented as compared to when no misleading details are provided. Thus, misleading post-event information can lead to profound errors in eyewitness testimony.

However, the comparison of the misinformation with the choice blindness paradigm indicates commonalities between the paradigms. That is, in choice blindness the manipulated outcome is presented after the original choice, as is misinformation 
after the original event. Thus, factors that moderate the misinformation effect could be equally effective for choice blindness. For example, misleading information effects increase as a function of the delay between the witnessed event and exposure to misinformation, as the memory of the original event becomes weaker over time (Loftus et al., 1978). Furthermore, whereas participants are easily influenced by misinformation that is provided by a credible source, they resist suggestions that are provided by a source who lacks credibility or a person who is perceived as having the intention to mislead (Dodd \& Bradshaw, 1980; Underwood \& Pezdek, 1998). In addition, the magnitude of the effect is subject to subtle social cues, such as the perceived power and social appeal conveyed by the accent of the person providing the misinformation (Vornik, Sharman, \& Garry, 2003). Finally, warning people about the fact they might have been exposed to misinformation can help to resist or even reverse the negative impact of the effect (e.g., Oeberst \& Blank, 2012). Therefore, consideration of these factors may provide useful insights about the boundary conditions and the relation between misinformation and choice blindness.

\section{Lineup Fairness}

Law enforcement agencies employ lineups as a method of presenting the suspect to the eyewitness. The proper and fair construction of a lineup is essential as it can affect the criterion setting and the decision making strategy an eyewitness adopts. A lineup consists of one suspect and a number of known to be innocent persons who are called foils. Foils are placed in the lineup because they enable the investigator to test whether the witnesses rely on their memory when making an identification decision, rather than deciding randomly about the guilt of the suspect (Luus \& Wells, 1991). Six alternative outcomes are possible as a result of a lineup procedure. These outcomes can be found in Table 1.1. The lineups where the suspect is identical with the perpetrator are called target-present and those where the suspect is innocent are called target-absent lineups.

Table 1.1 | Possible Lineup Outcomes.

\begin{tabular}{lccc}
\hline \multirow{2}{*}{ Lineup } & & \multicolumn{2}{l}{ Eyewitness response } \\
\cline { 2 - 4 } & Suspect & Foil & Lineup Rejection \\
\hline Target-present & Correct ID & Foil ID type 2 & False rejection \\
Target-absent & False ID & Foil ID type 1 & Correct rejection \\
\hline
\end{tabular}

Note. Foil identification (ID) type 1 is considered as a correct rejection since the perpetrator is not present and the eyewitness did not pick the innocent suspect. Foil ID type 2 is considered a false rejection since the perpetrator is present but was not picked. Source: Charman \& Wells (2007). 
Lineup Instructions

Prior to the actual presentation of the lineup, the police officer administering the lineup ought to instruct the eyewitness about the procedure. However, the police officer may be perceived as an authority figure, allowing two forms of social influence to occur (Steblay, 1997). The first concerns informational aspects and specifically the assumption that police would not present a lineup if they were not certain about the perpetrator. Hence, eyewitnesses may infer that the perpetrator must be present in the lineup and use that inference to make a positive identification. The second has to do with conformism, as the witnesses may feel pressure to pick someone in order to fulfil their duty to be a "good" witness. These feelings can be further reinforced through biased instructions which imply that the perpetrator is present in the lineup (e.g., "We believe that the perpetrator is present in the lineup") while, at the same time, may prevent the witness to reject the lineup (e.g., "Which of these lineup member is the perpetrator?"). Hence, with biased instructions, the false identification rate increases compared with lineups with unbiased instructions (Clark, 2005; Malpass \& Devine, 1981; Steblay, 1997). Therefore, instructions provided to the witness prior to the lineup presentation should be designed to control for the danger posed by those influences. This includes the verbal, explicit explanation that the perpetrator may or may not be present in the lineup, as well as the possibility to reject the lineup if none of the lineup members is recognized as the perpetrator. Thus, in the context of eyewitness identifications, great care is taken to avoid eyewitnesses feeling forced to choose a lineup member as this is considered to be biased and bad practice for identifications. By contrast, the choice blindness paradigm entails forced choice decisions. That is, participants are required to choose one of the alternative outcomes. Therefore, manifestations of blindness in the eyewitness setting may vary depending on the liberty witness are given to select a member of a lineup or reject it entirely.

\section{Investigator Bias}

While conducting research it is possible that the expectations of the researcher affect the experimental outcome (Rosenthal, 2002). Indeed, researchers may fall prey to confirmation bias in an attempt to prove their hypothesis by, consciously or unconsciously, creating the appropriate conditions. Analogously, the police investigator who holds a hypothesis about a suspect is susceptible to the same biases that apply to researchers (Wells \& Luus, 1990). In order to confirm their hypothesis the investigators may reveal their intentions to the witness nonverbally, for example through a nod or by avoiding to ask questions that disconfirm the hypothesis; or verbally, for example through the given instructions which may increase the tendency of the witness to choose. Additionally, their strong motivation to solve the case and confirm their 
hypothesis may tempt them to alternate eyewitness identifications or statements. We return to this point later in the introduction.

A simple way to examine the impact of confirmation bias and to avoid it altogether is to leave the investigator blind to the hypothesis at hand. In other words, the officer administrating the lineup should be unaware of the position of the suspect in the lineup and at the same time the witness should be informed about the administrator's lack of knowledge (double-blind method). Examining the effect of administrator knowledge on identification accuracy, Phillips, McAuliff, Kovera, and Cutler (1999) reported that when the administrator knew who the suspect was, the false identification rate was significantly increased compared with when this was not the case. Furthermore, Greathouse and Kovera (2009) examined the probability of a choice to be correct when the witness was informed about the administrator's lack of knowledge (single-blind method) compared with when the witness was informed about the administrator's lack of knowledge and the administrator was, indeed, unaware of the suspect's position (double-blind method). The superiority of the double-blind method was obvious, as the probability of a choice to be correct was two times higher using the double-blind than the single-blind lineup presentation method. Taken together, these findings indicate that not only the behaviour, but also the expectations of the witness about the behaviour of the lineup administrator can largely influence eyewitnesses' decision making strategies.

\section{Post-Identification Feedback}

A different method of influencing a witness is via post-identification feedback. The provision of either positive or negative feedback can influence witnesses in their certainty about the decision made (Bradfield, Wells, \& Olson, 2002; Charman \& Wells, 2012; Douglass \& Steblay, 2006). Specifically, Wells and Bradfield (1998), who examined the influence of positive feedback (e.g., "Right, this is the perpetrator") after the identification, report that witnesses who had been given positive feedback were more confident about their identification decision, reported better viewing conditions, said they had paid more attention, and were more willing to testify in court compared with people who were given no feedback. The effect is not only robust and reliable (Douglass \& Steblay, 2006), but is also persistent even when the feedback or the measures of its effects are delayed (Neuschatz et al., 2005; Wells, Olson, \& Charman, 2003). Likewise, the provision of negative feedback can lead to deflated confidence regarding the identification decision (Charman \& Wells, 2012), although finding the effect of disconfirming feedback has often been associated with great difficulty (e.g., Wells \& Bradfield, 1998; Wells et al., 2003).

The hazard of post-identification feedback may not be readily visible. Such feedback, however, has the potential to influence the relationship between identification 
accuracy and confidence. Specifically, confidence is considered a reliable estimator of memory strength. Sporer et al. (1995), in their meta-analytic approach, reported a high correlation of identification accuracy and confidence $(r=.41)$ for witnesses who chose from a lineup (i.e., choosers). In other words, the more confident the witness feels about the decision made, the greater is the probability that it is actually an accurate one. More recent studies have confirmed the strength of the relation for choosers (Brewer, Keast, \& Rishworth, 2002; Sauerland \& Sporer, 2009; Sporer et al., 1995; Weber \& Brewer, 2004). However, for nonchoosers, the confidence-accuracy relation is found to be negligible (Brewer \& Wells, 2006; Sauerland \& Sporer, 2009; Sporer et al., 1995), except when showup is used as a presentation method (Sauerland, Sagana, \& Sporer, 2012).

Post-identification feedback and other external factors affect the relationship between confidence and memory and hence diminish the diagnostic value of confidence ratings. Positive feedback, for instance, increases the post-hoc confidence of witnesses without increasing their accuracy, therefore reducing the postdictive value of confidence (Bradfield et al., 2002). Finally, as the provision of feedback impacts metacognition it can in turn affect the decision making criterion of the eyewitness and as such the beliefs about one's own memory strength.

In the context of eyewitness identifications the outcome of choice blindness manipulations may be strongly related to post-identification feedback. Specifically, the presentation of the manipulated outcome could be seen as disconfirming the original decision. Hence, blindness manipulations may exert similar effects on eyewitness identifications decisions as post-identification feedback.

\section{It's Your Choice! - Or Is It Really?}

Earlier we reviewed two prominent theories accounting for witness decision making strategies as well as factors that affect decision making. Along with the ability to make a decision, witnesses' competence to maintain and defend those decisions is of crucial importance. That is particularly true considering that eyewitnesses are often required to take the stand in support of their testimony in court. The ability to stand by a decision was not considered to be a problem until recently. In fact, extensive research on the so called forced-choice paradigm speaks in favour of a choice-induced preference (e.g., Brehm, 1956; Egan, Bloom, \& Santos, 2010; Lieberman, Ochsner, Gilbert, \& Schacter, 2001; Salti, El Karoui, Maillet, \& Naccache, 2014). That is, following a forced-choice decision, people tend to increase preference ratings of the chosen alternative and decrease those of the rejected option (i.e., spreading of alternatives). Furthermore, several studies have revealed that people tend to remember in ways that support their past decisions by, for example, attributing more positive features to the chosen compared with the rejected alternative (Henkel \& Mather, 2007; Mather 
\& Johnson, 2000; Mather, Shafir, \& Johnson, 2000b, 2003). This bias in favouring the chosen option in memory has been found in eyewitness identification decisions as well. When asked to make a lineup identification after having selected a suspect from mug shots, eyewitnesses exhibit a commitment effect that results in supporting their original decision, at the expense of identification accuracy (e.g., Brigham \& Cairns, 1988; Dysart, Lindsay, Hammond, \& Dupuis, 2001; Gorenstein \& Ellsworth, 1980).

Nevertheless, choice blindness suggests that people can be oblivious of surreptitious changes in the outcome of their decisions and confabulate introspective arguments for a choice they never made (Johansson et al., 2005). In fact, it seems that we may overestimate people's, and hence eyewitnesses', consistency when it comes to their choices. Given that humans are notoriously bad at noticing changes to visual scenes, when these changes are accompanied by some other visual disturbance (Simons \& Levin, 1998) this should come as no surprise. Therefore, in order to understand eyewitnesses' decision making, we ought to investigate how the apparent limited capacity to introspect on our past decisions may affect testimonial accuracy and whether the effect sides with estimator or system variables in the further expense of testimonial accuracy.

\section{From Change Blindness to Choice Blindness}

In 1998 Simons and Levin conducted a field study which portraits the limited capacity of the human cognitive apparatus (Simons \& Levin, 1998). The researchers, while pretending to be tourists, approached pedestrians in a local city park asking for directions. During the conversation, two men carrying a door passed between the experimenter and the pedestrian. At that point the person asking for directions changed places with one of the persons carrying the door. This change in identity went unnoticed by 7 $(46.6 \%)$ of the 15 participants. This phenomenon has been termed change blindness and refers to the difficulty that observers have in noticing changes to visual scenes (e.g., the person swap), when the changes are accompanied by some other visual disturbance (e.g., the passing of the door).

Over the past decade, the change blindness phenomenon has been replicated many times (e.g., Davis, Loftus, Vanous, \& Cucciare, 2008; Levin, Simons, Angelone, \& Chabris, 2002). To most of us, it seems bizarre that people could miss obvious changes while they are paying active attention. However, to detect those changes, attention must be targeted to the changing feature. Hence, the change either needs to be expected or people's attention needs to be guided towards the changing feature (Simons \& Rensink, 2005; Williams \& Simons, 2000). Rather than monitoring all of the visual details, people seem to focus their attention only on those features that are currently meaningful or important, ignoring those that are irrelevant to our current needs and goals (O'Regan \& Noë, 2001). Thus, at any given time, the representation 
of the world surrounding us is crude and incomplete, making it possible for changes or manipulations to go undetected (Chabris \& Simons, 2010).

Given the difficulty people have in noticing changes to visual stimuli, Johansson, Hall and colleagues (2005) wondered whether people may at times be blind to changes that involve not the visual scene, but their own intentions, actions, and introspections. To address this issue, Johansson et al. (2005) modified the change blindness paradigm to apply it to a decision making task. They showed participants pairs of female faces and asked them to choose which one they found most attractive. After a decision had been made, participants were presented with the selected face and were asked to explain their choice. However, in three of 15 trials, their choice was manipulated and participants were shown the very face they had not chosen previously. An overwhelming majority (87\%) failed to detect the manipulated trials, suggesting that people are often blind to manipulations of their choices. Johansson et al. (2008) termed this effect choice blindness. Figure 1.1 represents the procedure of the classical choice blindness paradigm for binary decisions and that of a later developed variant, incorporating ratings.
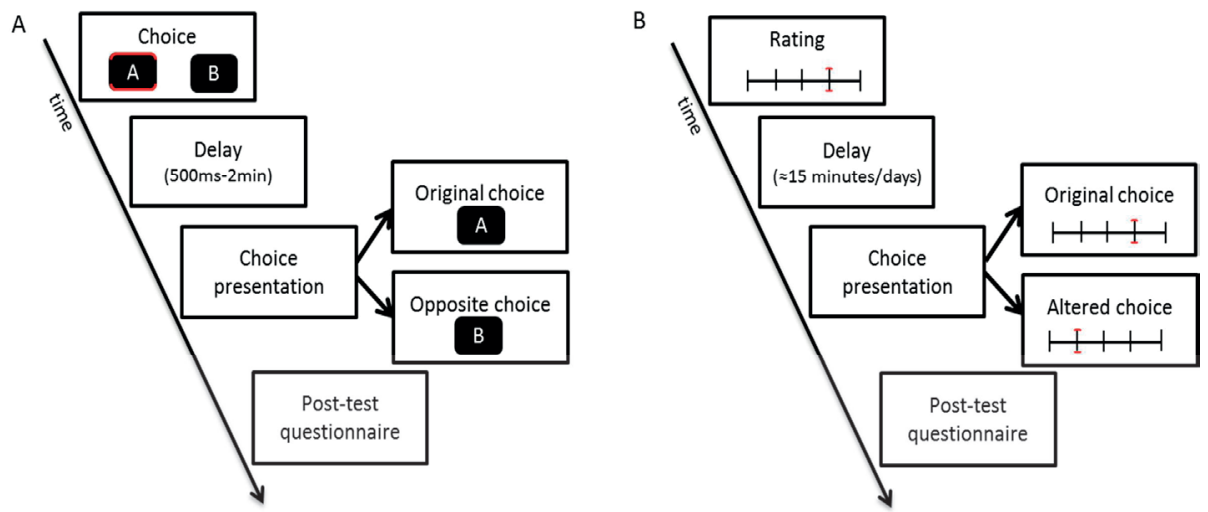

Figure 1.1 | Procedure of the classic binary choice blindness task (Panel A) and its variant incorporating ratings (Panel B). Participants first indicate a preference; provide a frequency or intensity judgment. Then, they are presented either with their original response (non-manipulated trial) or with a response that is inconsistent with their own (manipulated trial) and then they are asked to explain their answer. Finally, participants are informed about the possibility of a manipulation via a post-test questionnaire, and are asked to indicate if this was true for them.

A critical reader may argue that participants might simply have refrained from revealing that they noticed the manipulation. However, the fact that many participants noticed one of the changes, but not the other two, suggests that this was not the case. To examine whether choice blindness also occurs in other situations, Hall and 
colleagues (Hall, Johansson, Tärning, Sikström, \& Deutgen, 2010) invited supermarket customers to sample two different kinds of jams and teas. After participants had tasted or smelled both samples, they indicated which one they preferred. Subsequently, they were purportedly given another sample of their preferred choice. In reality, however, for half of the trials these were samples of the non-chosen jam or tea. Again, two-thirds of the participants were blind to this manipulation. Based on these findings, Hall et al. (2010) proposed that choice blindness is an effect that occurs not only for choices involving visual material, but also for choices involving gustatory and olfactory information.

Inspired by these findings, Merckelbach, Jelicic, and Pieters (2011b, Experiment 2) investigated choice blindness for intensity ratings of psychological symptoms. Their participants had to rate the frequency with which they experienced a wide range of common symptoms (e.g., anxiety, lack of concentration, stress, headaches etc.) on a five-point Likert scale. Prior to a follow-up interview, the researchers inflated the ratings for two symptoms by two scale points. For example, when participants had rated their feelings of shyness, as 2 (i.e., occasionally), it was changed to 4 (i.e., all the time). More than half (57\%) of the 28 participants were blind to the symptom rating escalation and accepted the rating as their own. This demonstrates that blindness is not limited to preference selections (Hall et al., 2010; Johansson et al., 2005), but can also occur for intensity and frequency ratings that rely on long-term episodic memory.

More recently choice blindness has been tested in the context of moral attitudes with striking results (Hall, Johansson, \& Strandberg, 2012). After having reported their opinion on 12 foundational principles (e.g., "It is more important for a society to promote the welfare of the citizens than to protect their personal integrity?") or 12 current moral issues (e.g., "Large scale governmental surveillance of e-mail and Internet traffic ought to be permitted as means to combat international crime and terrorism") on a nine-point bidirectional scale, participants were exposed to a reversal of two of their stated moral attitudes. Once again, more than two thirds of the participants $(69 \%)$ were blind to at least one of the performed manipulations. The authors considered their findings to lend support to models holding that moral decisions are achieved through intuition and are justified through post-hoc rationalization (Haidt, 2001). They concluded that not only moral attitudes are vulnerable to alternations, but also that choice blindness manipulations could jeopardize the formation of moral attitudes.

Similarly, Sauerland et al. (2013) tested blindness for one's own history of normviolating behaviours. Participants were presented with an 18-item questionnaire about their history of norm violations (e.g., "Have you ever stolen a bike?") and indicated how often they had displayed each behaviour on a 4-point Likert scale. Participants were later interviewed about four of those items, two of which had covertly 
been manipulated. As expected, a substantial proportion (25\%) of participants failed to notice changes of their own ratings. Importantly, blindness varied as a function of the interval between completing the questionnaire and being interviewed about the manipulated outcome. Participants who were presented with the manipulated outcome after 10 minutes (Experiment 1) were more likely to notice the change in their response than participants who were presented with the manipulation after a week (Experiment 2; 75\% and 55\%, respectively). Thus, the longer interval resulted in an increase of blindness.

Apart from the immediate impact, accumulating evidence indicates that choice blindness may continue to influence decision making beyond the time of the initial manipulation. For example, in their most recent attitude formation study, Johansson, Hall and colleagues (Johansson, Hall, Tärning, Sikström, \& Chater, 2013) observed that when participants had to indicate their preference again in a second round directly following the first one, the initially rejected faces were chosen more frequently in the second selection round. Additionally, the perceived attractiveness of these faces was increased. Likewise, in their study on symptom escalation and choice blindness, Merckelbach, Jelicic, \& Pieters (2011a) found that blind participants were more likely to report increased symptom ratings at a re-test one week later. Similarly, Sauerland, Schell and colleagues (2013) noted that four weeks after the initial interview, blind participants tended to modify their ratings on how often they had committed certain norm-violating behaviours in a way that was consistent with the manipulation. The effect indeed resembles the "free-choice paradigm" effect (i.e., the tendency of people to come to prefer what they have chosen more and to view the rejected outcome less favourably), hence speaking in favour of a common mechanism of the two effects. We will return to this point in the following section.

Together, these studies suggest that choice blindness can occur for a wide variety of decisions, including those that are important and highly self-relevant. Recent studies have extended the application of the choice blindness paradigm further to the linguistic domain for spoken decisions (Lind, Hall, Breidegard, Balkenius, \& Johansson, 2014) and as an instrument to study confabulation about mental states in obsessive-compulsive disorder (Aardema et al., 2014). Table 1.2 provides a summary of all choice blindness studies including the type of the decision, the procedure that was followed and the overall concurrent and retrospective blindness rates. Given the diverse type of decisions these studies comprise, from this point onwards we would use the term blindness phenomena interchangeably with the term choice blindness. A careful reader may notice that the magnitude of the effect is reduced as the choice moves from trivial decisions of facial attractiveness to fundamental questions concerning moral attitudes and norm-violating behaviours (see Table 1.2). The possibility that the discrepancy results from the differences in the experimental designs cannot 


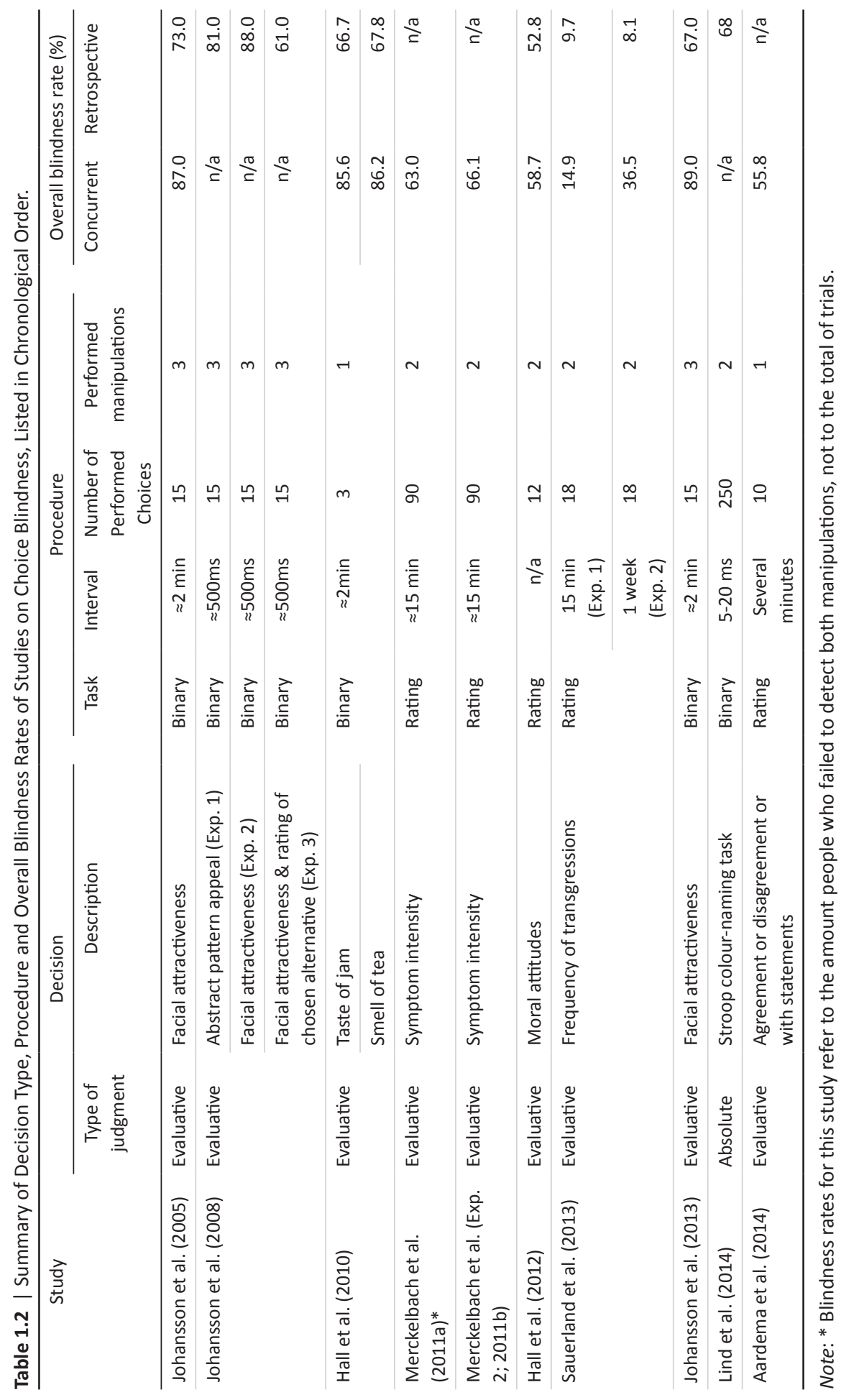


be ruled out. Nevertheless, the findings imply that the universality of the phenomena is limited by human rationality and this could speak to the mechanisms underlying the effect.

\section{The underpinnings of choice blindness}

Despite the fact that choice blindness is widely replicated, little is known about the factors underlying the effect. An initial attempt was made to link choice blindness to Nisbett and Wilson's (1977) classic article on people's lack of introspective access (Johansson, Hall, Sikström, Tärning, \& Lind, 2006). According to this view, participants base their reports about the cognitive processes underlying their choices (e.g., Why do you like chocolate? Why did you become a doctor?) on a priori, implicit causal theories about the probability of a specific impetus (e.g., feature, experience, life event) to have caused the given response. In fact, Johansson, Hall and colleagues considered choice blindness to be an experimental paradigm to test empirically Nisbett and Wilson's notion that "there may be little or no direct access to higher order cognitive processes" (1977, p. 231; Johansson et al., 2006).

Later, Hall and Johansson proposed that choice blindness may be related to posthoc rationalization and ultimately cognitive dissonance (Hall et al., 2012; 2011); an idea that is consistent with Nisbett and Wilson's original work. Specifically, after people have accepted a manipulated outcome, they may distort their memory for the selected outcome to reduce their dissonance with regard to the reasons guiding their decision. Notably, cognitive dissonance has been offered as an explanation for the tendency of individual to increase their preference rate for the original choice once the decision is taken. As a result, the self-deceptive effect of dissonance could only explain why participants confabulate reasons for a choice they (believe to have) made or why participants shift their subsequent responses in the direction of the manipulated information (e.g., Hall et al., 2010; Johansson et al., 2013; Sauerland, Schell, et al., 2013). However, it cannot explain why people fail to detect changes in the outcome of their decisions in the first place.

Considering the apparent similarity of choice blindness with misinformation, theories concerned with the misinformation effect may provide useful insights for choice blindness. Loftus (1979; Loftus \& Hoffman, 1989) suggested that the misinformation effect is caused by memory impairment. That is, the false information results in altering, erasing, or decreasing the accessibility of the memory of the original information. Hence, the original information is distorted by the presentation of the new information. Alternatively, choice blindness could result from the permanent alteration of the memory trace for the original choice after the presentation of the manipulated outcome. Yet, memory impairment cannot account for the decreased level of blindness as the importance of the decision increases. Nevertheless, considering that 
methodological differences across studies could account for these findings; memory impairment may be a valuable candidate to explain choice blindness.

Finally, a broader perspective has been adopted by Merckelbach et al. (2011b) who proposed that choice blindness results from ambiguity that consequently makes people susceptible to misinformation. Ambiguity may be inherent to the task, as it is for example with evaluative choices (Hall et al., 2010; Johansson et al., 2005) which are less clear cut than tasks involving an objectively correct or incorrect outcome. In studies involving self-reported ratings, the items being measured are not easily defined (such as the intensity of one's symptoms), making changes difficult to detect. Ambiguity can also be introduced by increasing the time between the decision and the presentation of the chosen item as illustrated in Sauerland, Schell, et al.'s (2013) study on norm-violating behaviours. Hence, ambiguity theory may provide useful insights about the limiting conditions of the effect.

All in all, though the mechanism underlying choice blindness is yet to be revealed, the psychological literature on choice blindness shows that it is a robust effect that persists across different modalities and types of decisions. Thus, it seems that under certain circumstances our decisions may be sensitive to the manipulations of clever marketers, careless doctors, and maybe ruthless police officers. Therefore, we postulate that choice blindness could have dramatic implications for the eyewitness setting.

\section{Choice Blindness in Eyewitness Settings}

After identifying a suspect from a lineup, eyewitnesses are frequently required to identify them again inside the courtroom. Consider a situation where the lineup administrator - intentionally or unintentionally - writes down a choice other than the one made by the witness. Likewise, consider the situation where a police officer transcribes a witness's verbal report and alters some minor yet essential detail of the case. Given the current evidence on choice blindness, one might worry that a substantial proportion of eyewitnesses may not notice these changes. Furthermore, considering the negative impact of factors such as cross-race bias, biased instructions and investigator's bias on testimonial accuracy, we postulate an aggravated impact of blindness under such conditions. Additionally, the presentation of a manipulated identification decision or of a tempered witness report may be perceived by the witness as a form of post-identification feedback thus affecting metacognitive judgments. This could have devastating consequences for the defendant as $\mathrm{s} / \mathrm{he}$ might be prosecuted on faulty grounds and even get incarcerated as a result of cognitive biases and limitations of our cognitive abilities.

The aforementioned scenarios might seem somewhat farfetched. After all, what are the chances that an eyewitness is presented with a different person than originally identified? Or that witness' statements are altered? Although this question has not 
been examined to date, we think it would be naïve to believe that police officers are always accurate when they write down the outcome of a lineup. As a matter of fact, literature on medical adverse events shows that this type of error is notoriously frequent. For example, performing surgery on the wrong site or wrong person due to site marking failure (e.g., marking the left instead of the right leg for amputation) and scheduling errors (e.g., scheduling the wrong participant for angioplasty), is estimated to occur at a rate of 0.09 to 4.5 per10,000 surgical cases (Pham et al., 2012). Furthermore, an increasing body of research is concerned with procedural errors such as mismatches of fingerprints and biases due to contextual influences as a major source of miscarriage of justice as well (Kassin, Dror, \& Kukucka, 2013; Saks $\&$ Koehler, 2005). Additionally, and beyond the possibility of an honest mistake, we ought to consider the possibility of a deliberate act. In fact, according to the Northern California Innocence Project (NCIP) report on prosecutorial misconduct, the odds of a deliberate act are far from scarce (Ridolfi \& Possley, 2010). Specifically, from 2000 till 2007 the NCIP investigation identified 4,000 alleged misconduct cases. In 707 of these, courts explicitly established that the misconduct had been committed by the prosecutors. Among others this included actions such as deliberately mishandling, mistreating, or destroying evidence as well as failing to turn over or withholding exculpatory evidence from the defence.

To sum up, we are bounded by the limited capacity of our cognitive apparatus and our human nature, which is prone to mistakes and driven by, at times, unkind motives. Therefore, we believe that research examining choice blindness in the context of eyewitness testimony can be of profound relevance for criminal investigations.

\section{Outline of the Present Dissertation}

In light of these intriguing results on choice blindness and with their implications in the legal arena in mind, the present dissertation aims to: 1) establish the relevance of choice blindness in eyewitness testimony, 2) explore the moderating factors of the effect of blindness in eyewitness testimony, and 3) unravel the mechanisms underlying choice blindness. To that end, we modified the classical choice blindness paradigm and its variants (see Figure 1.1) to incorporate manipulations of eyewitness identification decisions and witness statements (i.e., multiple choice).

Next to the general introduction and the general discussion chapters, this dissertation is composed of four main parts. Part 1 describes laboratory studies that focus on establishing the occurrence of choice blindness in earwitnesses and eyewitnesses. In Chapter 2 we investigate whether choice blindness is relevant for earwitnesses by manipulating participants' choices on which of two voices they perceived to sound more criminal or sympathetic. We anticipated that blindness will be fostered because of the natural difficulty people have in recognising voices. Chapter 3 offers an in depth 
investigation of blindness for eyewitness recognition decisions through a series of five laboratory studies. In Experiment 1 we aimed to bridge blindness for evaluative and absolute decisions. In Experiments $2 a-c$ and 3 we explored elements that may be decisive for the occurrence of blindness such as sample characteristics and retention interval.

The second part of this dissertation is devoted to transferring the findings to the field. Inspired by the work of Simons and Levin (1998), we performed two field studies, one with and one without criminal content. Chapter 4 describes how, in our first attempt to examine blindness for eyewitness recognition decisions under realistic conditions, we approached pedestrians and asked them to identify two previously seen tourists from target-present lineups. In Chapter 5 we replicated and expanded on the first field study, using a live staged crime, both target-absent and target-present lineups and the option to reject the lineup.

Having established the effect in the laboratory as well as in the field, in Part 3 expand the application of the choice blindness paradigm to other eyewitness settings. Specifically, Chapter 6 focuses on the impact of cross-race bias on blindness for eyewitness identification decisions. We expected other-race identifications to result in higher blindness than own-race identifications due to the increased ambiguity of the former condition. In Chapter 7, we investigated the extent to which witnesses detect covert manipulations in their written statements for a variety of retention intervals. We hypothesized that blindness would be relevant for manipulations in witnesses' statements and the ability of participants to detect manipulations would reduce as retention intervals increase.

Part 4 provides a more in depth discussion of the potential moderators and the cognitive mechanisms underlying choice blindness. In this attempt we moved away from the forensic field. The reason for this is because witness decision making is a rather complex procedure where perceptual and cognitive processes side with social influences. The complexity of the witnesses setting, thus, makes it difficult to isolate single factors responsible for blindness. That pursuit is facilitated if one investigates choice blindness per se. Therefore, it is useful to employ a simple experimental design wherein we control for confounding factors. Note, however, that although this part provides a systematic approach to the issue of potential moderators, the pursuit and discovery of moderators is present across all chapters. Naturally, the mere application of the choice blindness paradigm to different settings reveals facilitating and limiting conditions for blindness phenomena. In Chapter 8 we examined the role of selfrelevance, defined as the extent to which self is implicated in a choice, as a moderator of choice blindness. In two studies, adolescent and child populations performed a binary choice task for high and low self-relevance conditions. We anticipated that high compared to low self-relevant decisions would generate lower blindness rates. 
Finally, in Chapter 9 we investigated memory deterioration and forgetting as a potential explanation of choice blindness. In a variant of the classic choice blindness paradigm, participants provided sympathy ratings for female faces. Having been faced with either an increased or a decreased sympathy rating, participants were asked to remember their original rating.

Finally, the general discussion provides an overview of the main findings presented in Chapters 2-9. Furthermore, we discuss the theoretical implications of the findings in the field of decision making as well as their practical contribution to the judicial system and the legal arena. 


\section{PART 1}

ESTABLISHING THE EFFECT 



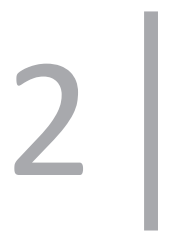

\section{Theoretical and legal issues \\ related to choice blindness for voices}




\section{ABSTRACT}

The aim of the study was to examine whether choice blindness occurs for auditory stimuli, namely voices. To that end, one hundred participants listened to three pairs of voices and had to decide each time which one they found more sympathetic or sounded more criminal. After they made a choice, participants were presented with the chosen voice again and had to match it to a face. However, during the second trial, participants were actually presented with the voice they had previously not chosen. Only $19 \%$ of the participants detected this change concurrently, an additional $10 \%$ detected it retrospectively. This indicates that choice blindness transfers to auditory stimuli. Whether participants had previously evaluated sympathy or criminality of the voices had no effect on choice blindness. The study shows that choice blindness is a robust effect that can also be elicited when auditory stimuli are employed. Implications for earwitness testimony and expert witnesses in the context of forensic speech analysis are discussed. 


\section{INTRODUCTION}

While most of us would probably agree that we are free to make choices every day and that we are aware of the choices we make, a series of experiments recently challenged this assumption. That is, Johansson, Hall, Sikstrom, and Olsson (2005) instructed 120 participants to decide which of two female faces they found more attractive. After they had made a decision, participants were handed the chosen face and were asked to motivate their decision. In 3 of the 15 trials, however, the participants' choice was manipulated. Here, a magical card trick was used in which participants ended up with the very face they had not chosen. Interestingly, only $13 \%$ of the manipulated trials were detected at the time of the manipulation. The authors dubbed this effect choice blindness. Also, another significant result from this study was that some participants included facial features in their choice motivation that the (manipulated) face in front of them did not carry (e.g., "she had a nice smile" while the person on the photo was not smiling). Others gave reasons that could only refer to the manipulated choice (e.g., refer to a smile when only the manipulated photo displayed a smile). Furthermore, during a post-test interview, $84 \%$ of the participants who failed to detect any of the manipulations stated that they thought they would be able to do so. This effect has been named choice blindness blindness (Johansson et al., 2005).

Since this first study on choice blindness, the effect has been demonstrated for a wide variety of visual stimuli such as female faces and abstract patterns (Johansson et al., 2008), as well as olfactory (smell of tea) and gustatory (taste of jams) stimuli (Hall et al., 2010). Furthermore, choice blindness seems to be a robust effect that persists across different stimulus similarities (although the effect tends to be stronger for more similar stimuli) and stimulus exposure times (e.g., $2 \mathrm{~s}$ vs. $5 \mathrm{~s}$ vs. unlimited; Johansson et al., 2005). Additionally, choice blindness is not limited to live administration as a real-world magic trick, but has also been found on a computerized test (Johansson et al., 2008).

To further test the robustness of the choice blindness effect, the current study examined whether this effect would also be present when using auditory stimuli, specifically voices. We expected to find the effect for two reasons. First, the effect has been demonstrated for three different modalities so far (Hall et al., 2010). We see no indication why auditory stimuli should be an exception. The second argument focuses more closely on the performance evolving around faces and voice perception. Although it is generally found that people are better at recognizing faces than at recognizing voices (e.g., Hanley \& Damjanovic, 2009; Stevenage, Howland, \& Tippelt, 2011), there are some parallels in the research findings looking at voices and faces. While humans are quite good at recognizing familiar voices, recognizing unfamiliar ones is much more difficult (e.g., Yonan \& Sommers, 2000). The same holds for rec- 
ognizing familiar versus unfamiliar faces (Johnston \& Edmonds, 2009). On the other hand, humans ability to discriminate voices is high - although not perfect (e.g., Kreiman \& Papcun, 1991), as it is for discriminating faces (Jenkins, White, Van Montfort, \& Mike Burton, 2011). In the light of these parallels and given the repeated finding of choice blindness for faces, we reason that the effect should occur for voices as well. If our hypothesis is supported, this could have important practical implications, namely for evidence given by earwitnesses and by expert witnesses in the context of forensic speech analyses.

\section{METHODS}

\section{Participants}

A total of $N=100$ (50 male, 50 female; $M_{\text {age }}=22.8$ years, $S D_{\text {age }}=1.8$, age range: $20-30$ ) native German speakers from Aachen and Maastricht University participated in the study. Participants were bachelor (87\%) and master (12\%) students with different majors. The participation was on voluntary basis; no monetary awards or course credits were provided. The study was approved by the standing ethical board of Maastricht University.

\section{Materials}

\section{Voice Pairs}

As stimulus material, we used three pairs of voices. The speakers, who were native male Germans, were instructed to read out loud, in normal tone and pace, three sentences (in German). These sentences were taken from the train magazine of the German Railways.

\footnotetext{
"In every ICE and IC there are coaches with express reservation or Bahn Comfort seats. Not all of these are always reserved. In ICEs of the first generation, these seats are located in the coaches with the numbers 6 and $7 "$.
}

The voices were recorded with a high-quality sound recorder (BOSS Digital Recorder Micro BR) and stored in an MP3 format. The audio track duration ranged from 13 to $17 \mathrm{~s}(M=14.5 \mathrm{~s})$ with an audio bit rate of $128 \mathrm{kbps}$.

Three pairs of voices varying in similarity (high, moderate, or low) were created. Voice similarity was established in a pilot study with $N=25$ native German speakers as participants who were second-year psychology students and received course credit for their participation. Specifically, participants listened to 21 pairs of voices and rated 
them on a 10-point Likert scale ( $1=$ very different; $10=$ very similar). Those voice pairs that were rated to be most similar $(M=6.19, S D=1.29)$ or least $\operatorname{similar}(M=4.00, S D$ $=1.32)$ were selected, as well as one voice pair of moderate similarity $(M=5.19, S D$ $=1.63)$. These three pairs of voices differed significantly in similarity, $t \mathrm{~s}(25) \geq 3.05$, ps $\leq .005$.

\section{Photos}

Six male head and shoulders photos were selected from the local database of Maastricht University. The size of the photos was $12.5 \mathrm{~cm} \times 16.0 \mathrm{~cm}$ and they were presented to the participants as printouts.

\section{Post-Test Questionnaire}

The post-test questionnaire was an adjusted version of the one used by Johansson et al. (2008) to match our stimuli. Furthermore, we added one more question. Specifically, participants were first asked whether they had noticed anything strange during the experiment, then whether they had noticed anything odd with the voices. If at this point no signs of detection were revealed, participants were then told that we planned a follow-up study where the choices sometimes would be switched and were asked if they would notice such a change. The answer to this question is referred to as the degree of choice blindness blindness in "Results" section. Finally, participants were informed that manipulations had actually taken place in the current study and were asked if they had noticed this. If participants responded with "yes", they were also asked in which trial they had noticed the change (additional item compared to Johansson et al., 2008).

\section{Marlowe-Crowne Social Desirability Scale (MCSDS)}

The MCSDS (Crowne \& Marlowe, 1960) is a 33-item true/false self-report inventory that aims to assess the individuals need for approval. The scores range from 0 to 33 , indicating a person's number of true responses, with a mean score of 13.71 (SD = 5.78) for normal adults (Crowne \& Marlowe, 1960). Examples of the items are: "I like to gossip at times" or "I never resent being asked a favour". The rationale of the MCSDS is that persons with higher need for approval tend to give more socially desirable responses than the average (Leite \& Beretvas, 2005). Here, we administered the MCSDS to examine whether choice blindness is associated with the tendency to act according to social demands, meaning that people might fail to report that they did notice the change in the manipulated trial due to their need for approval. 


\section{Gudjonsson Compliance Scale (GCS)}

The GCS (Gudjonsson, 1989) is a self-report inventory consisting of 20 true/false statements such as: "People with a lot of authority make me feel uncomfortable", "I give in easily when I am pressured", or "I try to please others". The scale is used to measure the tendency of people to conform to other people's requests. The scores range from 0 to 20. An average score of $8.9(S D=3.2)$ is considered to be typical for normal adults (Gudjonsson \& Sigurdsson, 2004). Similar to the MCSDS, the GCS was included to control for the possibility that participants were not blind to the manipulations, but rather complied with the presumed expectations of the experimenters.

\section{Design}

Similarity between the voices in the manipulated trial (high vs. low) was varied in a two-level one factorial between-subjects design. The detection rate of manipulated trials was the dependent variable. Two different measures of detection were used, namely concurrent and retrospective. Concurrent detection rate includes all participants who immediately noticed the manipulation when it had taken place. Retrospective detection additionally includes all those participants who reported that they had noticed the change of the voices in the post-test questionnaire.

\section{Procedure}

Data collection took place at two university libraries. Participants were approached and asked if they would like to participate in a study conducted by our University. All participants were tested individually. Upon agreement, participants signed the informed consent form and were randomly assigned to the conditions.

Then, participants received a written explanation of what the purpose of the study was (cover story). Some participants were led to believe that the aim of the study was to investigate whether features of a voice were associated with perceived sympathy in facial expressions. Other participants were informed that the university was involved in a research project that investigated whether auditory information could be beneficial for identifying criminals. Originally, this variation was meant to manipulate the perceived importance of the study as a second factor. However, a manipulation check showed that there was no difference in how important participants perceived the two conditions, $p=.593$. Therefore, we will not discuss this manipulation any further. Note that the two conditions also did not vary with regard to the choice blindness measures, $p s=1.000$.

After reading the cover story, participants listened to the first pair of voices. The audio stimuli were presented via a laptop. To minimize external sound distraction, high-quality headphones that covered the ears completely were used. Participants then rated each of two voices regarding their sympathy or criminality on a 10-point 
Likert scale ( 1 = not at all criminal/sympathetic; 10 = highly criminal/sympathetic). Hereafter, participants had to decide which voice they ultimately found more sympathetic or criminal by marking a checkbox. The chosen voice was then directly played once again. In accordance with previous studies (Hall et al., 2010; Johansson et al., 2005), we used the same clips on both occasions. Now, participants were given the task to match the voice to one of two photos that were presented in front of them. This was to ensure that participants would also listen closely the second time that they heard the voice. After participants indicated which picture matched the voice, the same procedure was followed for two more pairs of voices. On the second trial, however, the participants' choice was manipulated. Specifically, participants were presented with the voice they had not previously chosen. If the participant noticed the manipulation concurrently, the experimenter pretended having switched the voices by mistake and continued with the third trial. The order of presentation of the three voice pairs was moderate-high-low similarity for the high-similarity condition and moderate-low-high for the low-similarity condition. There was no condition in which the moderately similar voices were manipulated.

After the three trials were completed, the post-test questionnaire was conducted in order to assess whether the participants had detected the manipulation, but had for some reason not mentioned that. Hereafter, participants filled in the MCSDS and the GCS. Finally, participants were thanked and debriefed.

\section{RESULTS}

An alpha level of .05 was used for all inferential analyses. For non-parametric analyses of $2 \times 2$ contingency tables, phi is reported as a measure of effect size.

\section{Choice Blindness}

The overall concurrent detection rate was $19 \%(n=19)$, with an additional $10 \%$ ( $n$ $=10$ ) of trials being detected retrospectively. Thus, $29 \%(n=29)$ of the participants detected the manipulated trial in total. Of all $N=100$ participants, 63 perceived themselves as able to detect the manipulation and of the $n=71$ participants who did not detect the manipulation, 38 (53.5\%) thought they would be able to do so. Thus, considerable choice blindness blindness was observed.

Two chi-square tests with voice similarity (high vs. low) as independent variable and concurrent vs. retrospective detection as dependent variables were conducted. The results revealed a significant effect of similarity on concurrent detection, $\chi^{2}(1, N$ $=100)=5.26, p=.040, p h i=0.23$. As expected, concurrent detection was more likely to arise for less similar voices $(M=28.0 \%)$, than for more similar ones $(M=10.0 \%)$. 
However, for retrospective detection, there was no significant effect of similarity, $\chi^{2}(1$, $N=100)=2.38, p=.186$, $p h i=0.15$. Nevertheless, on a descriptive level, manipulations were detected more often for less similar $(M=36.0 \%)$ than for more similar voices $(M=22.0 \%)$.

\section{Choosing Patterns}

In the low-similarity condition, 18 participants chose the voice that was played first and 32 the voice that was played second. Similarly, in the high-similarity condition, 17 participants chose the voice that was played first and 33 the voice that was played second. This means that regardless of similarity condition, participants decided for the second voice in about two-thirds of the cases, whereas the voice played first was chosen in only about one-third of the cases. More interestingly, when participants chose the voice they heard first, they were less likely to discover the change than if they chose the voice presented second. This was true for both concurrent detection, $\chi^{2}(1, N=100)=6.18, p=.015, p h i=0.25$, and retrospective detection, $\chi^{2}(1, N=100)$ $=10.91, p=.001$, phi $=0.33$. Although no separate tests could be computed for the two similarity conditions due the small $n s$ in the individual cells, similar result patterns emerged for both conditions. Specifically, if participants chose voice 1 and were in the low-similarity condition, they spontaneously detected the change in only 2 of 18 cases (retrospective: 2 out of 16); for the high-similarity condition, it was 0 of 17 cases (retrospective: 1 out of 17 cases). If participants chose voice 2 in the low-similarity condition, they spontaneously detected the change in 5 of 33 cases (retrospective: 10 out of 33); for the high-similarity condition, it was 12 of 32 cases (retrospective: 16 out of 32 cases). These results may be due to recency effects and point to the role of short-term memory. Furthermore, the possible effects of distinctiveness will be explored at the end of the results section.

\section{Compliance and Social Desirability Measures}

It could be argued that some participants did detect the change during the manipulated trial, but did not say so as a result of a tendency to respond in a socially desirable way or to comply. To control for such effects, participants MCSDS and GCS scores were obtained. Choice blind participants did not have higher social desirability scores ( $M=14.3,95 \% \mathrm{Cl}[13.2,15.5])$ or compliance scores $(M=7.1,95 \% \mathrm{Cl}[6.2,8.0])$ than non-choice blind participants $(M=12.6,95 \% \mathrm{Cl}[12.4,16.4] ; M=9.0,95 \% \mathrm{Cl}[6.9,9.2]$, respectively), all $|t| s \leq 1.12, p s \geq .234,|d| s \leq 0.27$. These results contradict the idea that choice blindness can be attributed to social demands or compliance. 
Table 2.1 | Items for measuring attractiveness, distinctiveness, pitch of voice, and presence of accent/ dialect of the selected voice pairs.

\begin{tabular}{llll}
\hline & \multicolumn{1}{c}{ Item } & Anchor low (1) & Anchor high (10) \\
\hline Attractiveness & How attractive is this voice to you? & Not attractive at all & Very attractive \\
Distinctiveness & $\begin{array}{l}\text { How easy would you find it to } \\
\text { recognize this voice from a group } \\
\text { of people who are talking to each } \\
\text { other? }\end{array}$ & Very difficult & Very easy \\
Pitch of voice & How is the pitch of this voice? & Very low & Very high \\
Accent/dialect & $\begin{array}{l}\text { Does this person speak with an } \\
\text { accent/dialect? }\end{array}$ & No accent /dialect at all & $\begin{array}{l}\text { Strong accent/ } \\
\text { dialect }\end{array}$ \\
\hline
\end{tabular}

\section{Post Hoc Voice Ratings}

To explore possible explanations for the effects found, $N=18$ native German speakers with different occupations $\left(M_{\text {age }}=38.3\right.$ years, $S D_{\text {age }}=11.3$ ) rated the used voices regarding their attractiveness, distinctiveness, pitch, and presence of an accent. The wording of the items and the referring anchors of the employed 10-point Likert scales ranging from 1 to 10 can be found in Table 2.1. None of our three voice pairs differed with regard to perceived voice pitch or presence of an accent/dialect, $|t| s(17) \leq 1.50$, $p s \geq .152,|d| s \leq 0.36$. Furthermore, the presence of an accent was generally estimated as being small $(M=2.57, S D=1.78)$. Note that all speakers originated from the same region in Western Germany. The two high-similarity voices differed somewhat with regard to their attractiveness (Ms $=5.61$ vs. 4.28 ), $t(17)=2.15, p=.046, d=0.51$, whereas the two low-similarity voices differed with regard to their distinctiveness (Ms $=4.06$ vs. 5.50$), t(17)=-2.43, p=.027, d=-0.58$. All other comparisons were non-significant.

\section{DISCUSSION}

The aim of the current study was to replicate the choice blindness effect for auditory stimuli, voice fragments in particular. To ensure maximum comparability, we kept the design as parallel to previous choice blindness experiments as possible. As expected, a large proportion of participants (71\%) were blind towards the manipulation that occurred during one of the three trials. The observed $19 \%$ concurrent detection lies in the range of the $12-39 \%$ that have been found in facial choice blindness conditions (Johansson et al., 2008; Johansson et al., 2005). It is also comparable to the choice blindness rates found for olfactory and gustatory stimuli (Hall et al., 2010). Thus, our study replicates and extends previous work on choice blindness thereby showing that 
choice blindness is a robust effect that seems to operate in a comparable way for different senses.

While auditory stimuli seem to be affected by the choice blindness effect as much as other senses, our data also allow one tentative conclusion for a possible boundary condition of the effect. Specifically, the lack of similarity between the chosen versus manipulated stimuli seems to be a limiting factor. We found an effect of similarity for concurrent (but not retrospective) detection and previous authors reported such an effect for a limited selection of stimuli (Hall et al., 2010; but see Johansson et al., 2005). Hence, the degree of similarity is most likely the determining factor. The post hoc voice ratings, however, showed that our low-similarity voices did not only differ in similarity, but also in distinctiveness, whereas our high similarity voices did not. This, points to the possibility that differences in distinctiveness, possibly in addition to differences in similarity, may create a boundary condition for the effect. Previous choice blindness studies also have controlled for stimulus similarity, but not distinctiveness (Hall et al., 2010; Johansson et al., 2008; Johansson et al., 2006). Therefore, it is conceivable that the observed effects (or lack thereof) could be due to differences in distinctiveness. This might be an interesting issue to explore in future studies.

One argument that comes to mind when encountering choice blindness studies is that participants probably did notice the change but refrained from saying so. This could be because they were not confident enough to indicate the error or because they thought that declaring it during the experiment could spoil the results. In both cases, though, the straightforward thing to do during the post-test questionnaire would be to make mention of the recognition of the error then. Indeed, the demand characteristics during the post-test questionnaire should work against the choice blindness effect, not for it (Hall et al., 2010). Furthermore, the genuine surprise displayed by many participants after the debriefing is hard to accommodate with the idea that participants pretended not to have noticed the change (cf. Hall et al., 2010). Finally, the social desirability and compliance results speak against the interpretation that persons who are prone to the social demands of the situation are more prone to choice blindness. This is in agreement with Merckelbach, Jelicic, and Pieters (2011a) who found that 49 participants (63\%) were blind towards their own ratings given on two items of the Symptom Checklist-90 (Derogatis, Lipman, \& Covi, 1973), independent of social desirability scores.

What other factors could be capable of explaining the choice blindness effect as found in the current study? Possible candidates could be attention and short-term memory. One specific pattern in our results suggests a prominent role of short-term memory. In total, participants were presented with three auditory fragments: voice 1 (fragment 1), voice 2 (fragment 2), and then, depending on their choice again with voice 1 or 2 (fragment 3). Interestingly, participants who chose voice 1 in the crucial 
second trial were less likely to detect the change than participants who chose voice 2 , regardless of voice similarity or distinctiveness. In cases where the participant chose voice 2 , this voice should still have been in the phonological store of the phonological loop (Baddeley, 1992) when fragment 3 was played. However, if a participant chose voice 1 , this voice should already have been absent from phonological store due to time constraints of this store and due to the presentation of fragment 2 prior to the presentation of fragment 3. Accordingly, it is reasonable to assume that it was more difficult for participants to make comparisons of fragments 1 and 3 than of fragments 2 and 3. On the other hand, one may argue that participants who chose fragment 1 should have noticed that fragment 3 was identical to the preceding fragment 2 . This is where the role of attention may come to play. Participants may not have directed enough attention to the comparison between fragments 2 and 3 by the time they articulated their choice of fragment 1 and while they were busy matching fragment 3 to one of two faces. Thus, the interplay between short-term memory and attention could be responsible for the result pattern as found.

An alternative explanation could be that distinctiveness is the crucial player in the current findings. Distinctiveness has shown to be an important factor in face-recognition performance (Bruce, Burton, \& Dench, 1994; Shapiro \& Penrod, 1986; Valentine, 1991) as well as voice-recognition performance (Mullennix et al., 2011; Orchard \& Yarmey, 1995). In our design, the voices of one voice pair were always presented in the same order. For the low-similarity condition, this means that the less-distinctive voice was always presented first. Thus, it is conceivable that the finding that detection was better when the second voice was chosen is a distinctiveness effect rather than a recency effect. Nevertheless, the two voices in the high-similarity condition did not differ in distinctiveness. Yet, the result patterns of the two conditions show great analogies. If distinctiveness played a superior role in the choice blindness effect, we would have expected the results patterns of the two conditions to deviate more from each other. Nevertheless, we cannot exclude the possibility that distinctiveness plays a viable role in the choice blindness effect. Alternatively, recency effects and distinctiveness may add up. This will be for future studies to determine.

Although the two high-similarity voices differed in perceived attractiveness, we do not think this to be a major factor for the current results, because at least facial attractiveness has repeatedly been shown to be unrelated to memory performance (Sarno \& Alley, 1997; Wickham \& Morris, 2003). We are not aware of any studies that tested the effect for voices, but given other parallels in the performance revolving around the perception of faces and voices, we suspect that voice attractiveness does not have an impact on performance. This is, however, subject to empirical testing.

We will now turn to possible implications of our findings for evidence given by earwitnesses and by expert witnesses in the context of forensic speech analyses. 
Cases exist in which a perpetrators voice may be the only evidence available. This can concern any crime that occurs in the dark, via telephone, or that involves a masked perpetrator. In such cases, auditory identification testimony can be treated as direct evidence of identity and therefore can be presented in court (Clifford, 1980). After identifying a suspect from an auditory lineup, an earwitness may be required to identify him/her again inside the courtroom. Consider a situation where the lineup administrator - intentionally or unintentionally - writes down a different choice than the one made by the witness. Given the current results, one must worry that a considerable proportion of earwitnesses may not notice that change. This, of course, could have devastating consequences for the defendant. Because such effects have been demonstrated for facial lineups (Chapter 3; Sagana, Sauerland, \& Merckelbach, 2014b), it seems very likely that they would also occur for voice lineups. ${ }^{1}$

Let us take a closer look at the literature comparing face and voice recognition. In a series of studies, Hanley and colleagues have demonstrated that it is more difficult for people to retrieve semantic information about celebrities from a voice than from a face (Hanley, Smith, \& Hadfield, 1998), even when the face has been blurred (Damjanovic \& Hanley, 2007; Hanley \& Damjanovic, 2009). To control for the fact that people encounter celebrities faces more frequently than their voices (i.e., in the media), a recent study replicated the effect when using personally familiar peoples voices and faces (Brédart, Barsics, \& Hanley, 2009). These results have been interpreted such that the face-recognition system is more closely connected to biographical information stored in semantic memory than the voice-recognition system (Damjanovic \& Hanley, 2007). Another interpretation could be that people are better at distinguishing between faces than between voices (Brédart et al., 2009). In any case, both interpretations indicate that choice blindness for lineups as described above should actually be more pronounced for voices than for faces. This seems an important and interesting research question worth further exploration.

Our results may also be meaningful for forensic speech expert witnesses. These professionals often deal with different speech samples and have to decide whether two samples belong to the same person or not, thus linking suspects to certain crimes. This could involve speech samples collected in suspects houses (e.g., answering machines, telephones), recorded by the investigative teams (e.g., in cases of bomb threats), obtained in wiretap operations as well as obtained directly from existing suspects. Forensic speech analysts can provide the police with important leads in a

1 The issue may be raised that we tested for clip recognition rather than voice recognition since we used the same clips in both phases of the Experiment (choosing and pairing with face). This is not a problem for the point we make here, however. In fact, the considerable choice blindness effect we found while using the same clips speaks to the prominence of the effect and we would expect the effect to be even stronger if different voice clips were used. 
case and their testimony can eventually play a vital role during trial. Although the paradigm used in the current study deviates somewhat from the forensic speech analysis setting, our results might apply nevertheless. What if speech analysts are choice blind to auditory stimuli, just like the participants in our study? This would obviously question the validity of the evidence given by such experts. Although speech identification experts perform significantly better at identifying speakers than nonexperienced persons (Schiller \& Köster, 1998), our speculative guess is that the effect would not disappear completely. Further, these experts are unlikely to be questioned due to their expert status. Therefore, such a bias is likely to go unnoticed and execute a devastating impact on innocent suspects and the legal system as a whole. Future research should address these issues.

To conclude, the current study has successfully shown that the choice blindness effect is present for auditory stimuli. In our view, its application to the legal system opens a new interesting and important field that can inform us on how to avoid biases in legal procedures. 



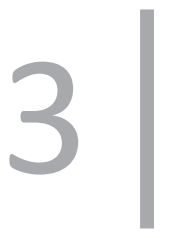

"This is the person you selected.": Eyewitnesses'
blindness for their own facial recognition decisions 


\section{ABSTRACT}

The aim of the current research was to identify conditions under which choice blindness in facial recognition decisions occurs. In five Experiments, participants watched four mock crime videos and made choices that were either evaluative (Experiment 1 ) or absolute in nature (Experiments $2 a-c, 3)$. When participants were subsequently asked to motivate their choice, they were sometimes presented with choices they had not made. For evaluative decisions, concurrent (27\%) and retrospective blindness rates (21\%) were relatively low compared with previous studies. For absolute decisions, choice blindness rates varied, depending on when exposure to the manipulated outcome took place (immediate: concurrent 32-35\%, retrospective 0-6\% [Experiments 2a-c]; $48 \mathrm{hrs}$ delay: concurrent $68 \%$, retrospective 39\% [Experiment 3]). We argue that blindness for facial recognition decisions is more likely for evaluative decisions and for longer intervals between decision and manipulation, but also for conditions of increased task complexity, which we interpret in terms of ambiguity. 


\section{INTRODUCTION}

In many western countries, eyewitnesses first identify the suspect from a lineup at the police station and later confirm their decision during a court proceeding. Thus, at trial, eyewitnesses have to testify in support of their earlier decision. Consider though the situation where the lineup administrator (intentionally or unintentionally) wrote down a different decision than the one made by the witness. Would eyewitnesses notice a change in their identification decision when they are later interviewed about it during, for example, a court session? The case of Bernard Maughan (Wolchover, n.d.) is an example of such a case. Specifically, the eyewitness in this case made an identification by saying "I think it's number six" in the presence of his solicitor. However, the administrator wrote down "I think it's number seven" (who happened to be the suspect) and read back the utterance to the witness. At that point, neither the eyewitness nor the solicitor demurred. As a result, the official record mentioned another than the actual identified lineup member. Subsequently, the defendant was charged and released on bail. It was only on the appeal, two years later, that the solicitor of the defendant spotted the miscommunication while reviewing the identification tapes. This appears to be an incident of choice blindness in the setting of eyewitness identification.

Choice blindness refers to the difficulty people have in detecting manipulations of a choice they previously made and their tendency to confabulate introspective arguments for the very choice they did not make (Johansson et al., 2005). In a first demonstration of choice blindness, Johansson and colleagues (2005) asked participants to choose which of two female faces they found more attractive. After they had made a decision, participants were presented with their choice and were asked to explain the reasons behind their decision. Using a magic card trick, three of the 15 trials were manipulated such that participants actually ended up with the non-chosen face. Participants were blind to 308 (87\%) of the 354 manipulated pairs. However, when participants were given a description of the procedure and were asked whether they would have noticed such choice manipulations, the vast majority of them were confident that they would have done so. Johansson et al. (2005) termed this metacognitive bias choice blindness blindness.

Inspired by these findings Sagana, Sauerland, and Merckelbach (Chapter 4; 2013) examined choice blindness for eyewitnesses' facial recognition decisions. Specifically, pedestrians in a European city were engaged in a conversation with two experimenters who pretended to be tourists. Shortly thereafter, the pedestrians were asked to identify the two experimenters from separate simultaneous photo lineups using a forced-choice recognition decision format. Subsequently, they were confronted with their selection and asked to motivate their decision. However, for the second target, 
the chosen lineup member was swapped with a previously non-identified member. Interestingly, $68.3 \%$ of the pedestrians failed to immediately report that they noticed the change (i.e., concurrently). Even after the end of the experiment in a post-test questionnaire (i.e., retrospectively) $39.8 \%$ of the participants were blind to this identity manipulation. Additionally, Sagana et al. (Chapter 4; 2013) reported that participants who made an accurate lineup decision were more likely to retrospectively detect the manipulation than participants who made an erroneous recognition decision. Hence, superior recognition performance was associated with higher detection rates.

While blindness phenomena have been demonstrated repeatedly, its border conditions have been an under-explored facet. Investigating these conditions is important because it may shed new light on factors that affect choice blindness in settings other than preference, such as the eyewitness identification setting. Drawing from the negative association between recognition performance and blindness, the effect may be conceptualized as a manifestation of weak memory. Indeed, memory deterioration for one's own decisions may foster blindness effects, as demonstrated by Sauerland, Schell, et al. (2013). Nevertheless, other studies showed that participants can be blind to changes in the outcome of decisions that they themselves made only few minutes before the presentation of the manipulated outcome (Hall et al., 2012; Hall et al., 2013; Johansson et al., 2005). Additionally, research on memory for choices suggests that people have an enhanced memory for a preferred than a rejected option, even when the grounds for this decision are unclear (Dellarosa \& Bourne Jr, 1984; Mather, Shafir, \& Johnson, 2000a). Thus, it appears that memory decay cannot fully explain choice blindness.

Poor processing of the original targets (Johansson et al., 2005) or the manipulated items (Chapter 2; Sauerland, Sagana, \& Otgaar, 2013) have also been ruled out as determinants of choice blindness. Furthermore, participants' compliance (Johansson et al., 2008; Johansson et al., 2005; Chapter 2; Sauerland, Sagana, et al., 2013), suggestibility (Merckelbach et al., 2011a; Sauerland, Schell, et al., 2013) or their tendency to react in socially desirable ways (Merckelbach et al., 2011a; Chapter 2; Sauerland, Sagana, et al., 2013) do not seem to modulate choice blindness. The degree of similarity between the original target and manipulated item has sometimes (Hall et al., 2010; Chapter 4; Sagana et al., 2013; Sauerland, Schell, et al., 2013), but not always (Johansson et al., 2005) been found to act as a moderating factor.

From another point of view, choice blindness is related to the constructive nature of preferences and self-persuasion (Johansson et al., 2011). Specifically, Johansson et al. (2011) argued that people have limited access to the reasons for their actions (see also Nisbett \& Wilson, 1977). As a consequence people can come to like what they are told or suggested to like. Endorsing choices suggested by others may generate a degree of cognitive dissonance, which people want to overcome (Festinger, 1962; Henkel \& 
Mather, 2007). Hence, they deliberate reasons to convince themselves about their assumed choice. Once the changed outcome has publicly been endorsed, people need to convince themselves that they, indeed, prefer that outcome. Relatedly, after people have accepted the manipulated choice, they may distort their memory for the selected outcome, through self-deception, in order to reduce dissonance.

Taken together, the literature on choice blindness shows that people have poor insight into their decisions, or flexible preferences that are easily influenced by external cues. Either way, the factors that affect the occurrence of the effect remain unclear. Nonetheless, the relevance to legal settings and the potential devastating consequences of choice blindness for (innocent) defendants are evident. With these considerations in mind, the experiments in this paper constitute a first exploration of the borderline conditions under which blindness for facial recognition decisions materializes.

Sagana et al. (Chapter 4 ; 2013) demonstrated the relevance of choice blindness for eyewitness decisions. However, the employed field study approach allows little control over the specific conditions that foster blindness in the eyewitness setting. The difficulty of the matter is demonstrated in the reduced blindness rates for recognition decisions found in that study compared with previous findings using visual stimuli (Johansson et al., 2005, 2008). One reason for this discrepancy could be the use of absolute versus evaluative decisions. Eyewitnesses base - or should base - an identification decision on their memory of the individual rather than an evaluative judgements. This is not to imply that eyewitnesses do not make evaluative decisions in real-life. In fact, in an archival analysis of persons descriptions, Sporer (as cited in Tuckey \& Brewer, 2003) found that about 5\% of the descriptors referenced perceived personality characteristics. In principle though, eyewitness identifications in an experimental setting can be tested against a ground truth and their outcome is absolute (i.e., correct or incorrect). Given the objective nature of identification decisions, one would intuitively expect that manipulations would readily be detected, implying little room for choice blindness. This is very much unlike for evaluative judgments about faces. A person may judge someone more or less sympathetic depending on their mood or on the context (Loewenstein \& Small, 2007). Accordingly, uncertainty about the evaluative decision made at an earlier point may easily occur.

If a missing evaluative component is responsible for the lower blindness rates in Sagana et al. (Chapter 4; 2013), it is reasonable to anticipate increased levels of blindness when an evaluative element is introduced to an eyewitness recognition task. To test this hypothesis, we employed a typical eyewitness recognition paradigm with the difference that participants, instead of selecting the perpetrator from a lineup, were asked to indicate which of the actors seen in a mock crime they found most sympathetic (Experiment 1). Additionally, to explore factors that might be critical for 
the occurrence of choice blindness, we performed a series of three studies (Experiment, 2a-c) where absolute decisions were required. Hence, in Experiments 2a-c, we employed a procedure similar to Experiment 1, with the difference that participants made a facial recognition decision rather than sympathy evaluations. We anticipated lower blindness rates for the absolute compared with evaluative decisions. Experiments $2 a-c$ also differed in the number of manipulations, mask duration (interval between 50 and $500 \mathrm{~ms}$ ), and sample characteristics in order to further investigate conditions that facilitate or inhibit blindness. Finally, in a fifth study (Experiment 3), we examined whether increased retention intervals between the recognition decision and the presentation of the manipulated outcome would affect blindness rates. We considered this important as it is unlikely that the identification procedure and the confrontation with one's decision at trial take place at the same day (Shermer, Rose, \& Hoffman, 2011). Thus, Experiment 3 employed a procedure similar to Experiment $2 \mathrm{a}$ with the exception that the manipulated outcome was presented $48 \mathrm{hrs}$ after the recognition task, which is a delay that adds to the ecological relevance of the study.

\section{EXPERIMENT 1}

\section{Method}

\section{Participants}

Thirty-four participants (19 males, 15 females) of various nationalities took part in the study $\left(M_{\text {age }}=25.2\right.$ years, $S D_{\text {age }}=5.2$, range: $\left.19-45\right)$. The majority (61\%) were Maastricht University students studying different majors (Law: 14.7\%, Psychology: 11.8\%, Mental Health: $11.8 \%$ ), whereas $38.2 \%$ were employees (private sector: $23.5 \%$, public sector: $2.9 \%$, other: $11.8 \%$ ). All reported studies were approved by the standing ethical board of the Faculty of Psychology and Neuroscience of Maastricht University.

\section{Materials}

Stimulus films. Four stimulus video fragments showing minor offences were displayed on a computer screen. The mean duration was $187 \mathrm{~s}$ (duration range: 160 $s-214 \mathrm{~s})$. Two of the fragments showed the theft of a wallet at a university cafeteria (Cafeteria1 \& Cafeteria2). The third fragment depicted the theft of a wallet in a bar (Bar) and the fourth one was about an assault attempt at a bus stop (Bus). In each video fragment, there were four actors: a perpetrator, a victim, and two bystanders. Actors were different for each film. The gender of perpetrators and victims was counterbalanced across videos. In two videos (Cafeteria1 and Bus), the perpetrator was a man and the victim a woman, whereas the reverse was true for the other two videos 
(Cafetaria2 and Bar). Each target was in sight for a minimum of $76 \mathrm{~s}$, with close-ups of 2 to $9 \mathrm{~s}$. All targets were shown from frontal and side views.

Post-Test Questionnaire. To examine whether participants had noticed our manipulations but refrained from mentioning it, we administered a post-test questionnaire that was adjusted from Johansson et al. (2008). Participants were first asked whether they had noticed anything strange during the Experiment and when they responded affirmatively, they were invited to provide details. If they reported they noticed the manipulation they were coded as retrospective detectors. Next, participants were given a description of an imaginary experiment in which some identifications were manipulated in such a way that the participant would end up with a photo that $s /$ he did not choose. To test choice blindness blindness (i.e., the meta-cognitive bias; Johansson et al., 2005) participants first had to indicate if they thought that they would notice such a change. Second, they were asked if they believed that we had carried out such a manipulation in the current Experiment. If participants responded affirmatively to this question they were again counted as retrospective detectors.

\section{Design}

In Experiment 1, we manipulated the preferred target in one of the four video fragments. Specifically, the decisions of the Bus or the Cafeteria 2 fragments were manipulated. Detection rates were measured both concurrently and retrospectively. Concurrent detection refers to participants who immediately noticed the manipulation after it had taken place. Retrospective detection, next to concurrent detection, additionally includes those participants who reported in the post-test questionnaire that they had noticed the change. In accordance with Johansson et al. (2008), any positive answer to the questions about noticing anything strange or noticing a manipulation taking place during the Experiment, were counted as retrospective detection.

\section{Procedure}

All parts of the experiment were presented on a 20 inch computer screen at a resolution of 1024 x 768 pixels using DMDX Display Software (Forster \& Forster, 2003). Participants were naïve to the actual purpose of the study and were tested individually.

After signing the informed consent form, participants were told that they would watch four video fragments and would act as eyewitnesses. Then, the first video was presented. After the end of the fragment, participants were shown a slide with photos of the actors they had seen during the video. Their task was to select which of these actors they found most sympathetic. Although the procedure is very similar to that in an identification experiment, participants' task was evaluative in nature.

For the three video fragments (Cafeteria1, Cafeteria2, and Bar) in which three actors were of one gender and only one actor of the other gender, we presented only 
the three actors of the same gender (in one row of three pictures). This was done to prevent an increased detection rate simply due to noticing the gender difference. For the fourth video, in which two male and two female actors were shown, all four actors were presented in two rows of two pictures.

After participants had made their choice, a 50 ms random pattern (i.e., mask) was displayed, followed by the presentation of the chosen actor. Now participants were asked to justify their decision. The same procedure was followed for all four videos that were presented either in the order Cafeteria1, Bar, Cafeteria2, and Bus or in the order Cafeteria1, Bar, Bus, and Cafetaria2. However, the decision for the video fragment presented last (either Bus or Cafeteria2) was manipulated and participants were confronted with a predetermined photo they had actually not selected. If participants' justifications included a comment indicating that the displayed photo did not correspond to their choice or that the program had made a "mistake", they were classified as concurrent detectors. Detectors frequently also verbally informed the experimenter about the change. No significant differences in detection emerged between the two orders for concurrent, $\chi^{2}(1, N=34)=1.89, p=.25$, phi $=0.24$, or retrospective detection, $\chi^{2}(1, N=34)=2.10, p=.21$, phi $=0.25$. Therefore, we will not discuss this factor further. At the end of the Experiment, participants were given the post-test questionnaire and were fully debriefed.

\section{Results and Discussion}

The concurrent detection rate was $73.5 \%(n=25)$, and retrospective detection was $79.4 \%(n=27)$. On the whole, $20.6 \%(n=7)$ of the participants were completely choice blind. Of these choice blind participants, $85.7 \%(n=6)$ said in the post-test questionnaire that they would be able to detect the manipulation. Thus, this group evidently exhibited choice blindness blindness.

The observed concurrent and retrospective choice blindness rates of the present study are relatively low compared with previous choice blindness studies for visual stimuli (Johansson et al., 2005, 2008). This may be due to the use of a video fragment with a specific plot. Specifically, it seemed that participants based their preference on the role of the target and their empathic feelings towards the victims and the bystanders. This is illustrated by the low preference rate for the perpetrators ( 3 of the 34 participants) than the victims and bystanders (91.2\%). That is, because their decision was often based on a clear category (e.g., victims), participants may have found it relatively easy to detect the manipulated outcome.

Most importantly and in contrast to our hypothesis, the choice blindness rates observed in Experiment 1 are lower compared with those reported by Sagana et al. (Chapter $4 ; 2013 ; 31.7-60.2 \%$ ). These findings do not speak to the idea that blindness rates depend on the decision type required (i.e., absolute versus evaluative). 
The difference in blindness rates across the two studies could be due to differences in methodology, as the present is a laboratory study whereas Sagana et al. (Chapter 4; 2013) performed a field study. That is, the various distracters that are inherent to real-life interactions may have increased the complexity of the task resulting in raised blindness rates (Chapter 4; Sagana et al., 2013) study. To test this notion, we examined whether choice blindness would be less prominent if participants performed a procedure similar to Experiment 1, however, with participants performing absolute facial recognition decision rather than evaluative decisions. To this end, we performed a series of three studies (Experiment, 2a-c). In addition to varying decision type (compared with Experiment 1), Experiments 2a-c differed in the number of manipulations, mask duration, and sample characteristics in order to test other conditions that could confine blindness for facial recognition decisions.

\section{EXPERIMENTS 2A-C}

\section{Method}

In Experiments 2a-c, participants made forced choice recognition decisions from target-present lineups. In session 1, participants watched four video fragments and made facial recognition decision for each of the four targets depicted in the films (i.e., 16 targets in total). After each decision, a mask was presented and subsequently the photo of the selected lineup member was displayed on the screen. However, some decisions (either 2 [Experiment 2a] or 4 [Experiment 2b/c]) were manipulated and replaced with a non-selected lineup member. Twenty-four hours later, participants returned to the lab for a second session and were asked to identify all perpetrators and victims again (i.e., eight targets in total). Session 2 was introduced to investigate whether the manipulations had an impact on participants' future decisions.

For Experiments 2a-c the same stimulus films, lineups, testing materials, and procedure were used. In Experiments 2a/b, we tested whether blindness for facial recognition decisions was affected by the number of manipulations and by mask duration. Specifically, we decreased the number of manipulations from four in Experiment 2a (4 manipulations) to two in Experiment $2 \mathrm{~b}$ and increased mask duration from $50 \mathrm{~ms}$ to $500 \mathrm{~ms}$. This was to test whether a large number of manipulations as well as the short mask duration would alarm participants and hence increase detection.

In Experiment 2c, we investigated whether our results were affected by our heavy reliance on bachelor psychology students as participants. Students may just have waited for some kind of manipulation to occur while they were tested, or they might have read or heard about the choice blindness effect during the course of their studies. Relatedly, the use of undergraduate student samples has been vigorously 
criticized among others as malleable to social influences (e.g., Sears, 1986). To rule out this possibility, Experiment $2 \mathrm{c}$ relied on participants who were currently not active in or had never been involved in academia. The procedure of Experiment $2 c$ was analogous to Experiment $2 \mathrm{~b}$, with the exception that there was only one session. This seemed appropriate since we were mostly interested in the blindness rates in session 1. Additionally, we asked participants in the post-test questionnaire how many manipulations they had detected in Experiment 2c.

\section{Participants}

In Experiments 2a/b, participants were 18 and 19 (26 females) students of Maastricht University, respectively, $\left(M_{\text {age }}=20.9\right.$ years, $S D_{\text {age }}=1.6$, range: $\left.19-25\right)$. The majority were Psychology students (73.0\%), one was a Mental Health student (2.7\%), whereas the majors of the remaining students (24.3\%) were not specified. In Experiment 2c, 20 (11 females) participants ( $M_{\text {age }}=41.5$ years, $S D_{\text {age }}=16.2$, age range: $19-64$ ) had varying professional backgrounds (private/public sector: $35 \%$, freelance: $20 \%$, household: $20 \%$, temporary employment: $25 \%$ ). Participation was voluntary. Student participants received course credit in return while for non-academics no monetary or other incentive was granted. All participants were naive to the purpose of the study and were tested individually.

\section{Materials}

Lineups. A total of 16 simultaneous, target-present photo lineups were created. To that end, head and shoulder photos were selected to match the description of the respective targets. Each lineup was presented on a $2 \times 3$ array format. Thus, each lineup included one target (i.e., perpetrator, victim or bystander) and five distractors. The size of the photos, as presented on the computer screen, was $9.0 \mathrm{~cm} \times 10.0 \mathrm{~cm}$. Only target-present lineups were administered and participants were not allowed to make rejections. We opted for this procedure because we wanted to test for the existence of choice blindness for lineups selections (rather than rejections). Note that other phenomena such as the feedback effect (Wells \& Bradfield, 1998, 1999) or the own-race bias effect (for a complete review see Meissner \& Brigham, 2001) were originally studied using a similar approach.

\section{Design}

In Experiments 2a/b, we manipulated either four or two of the 16 lineups. Table 3.1 summarizes the manipulations per Experiment. Detection rates were measured both concurrently and retrospectively. 


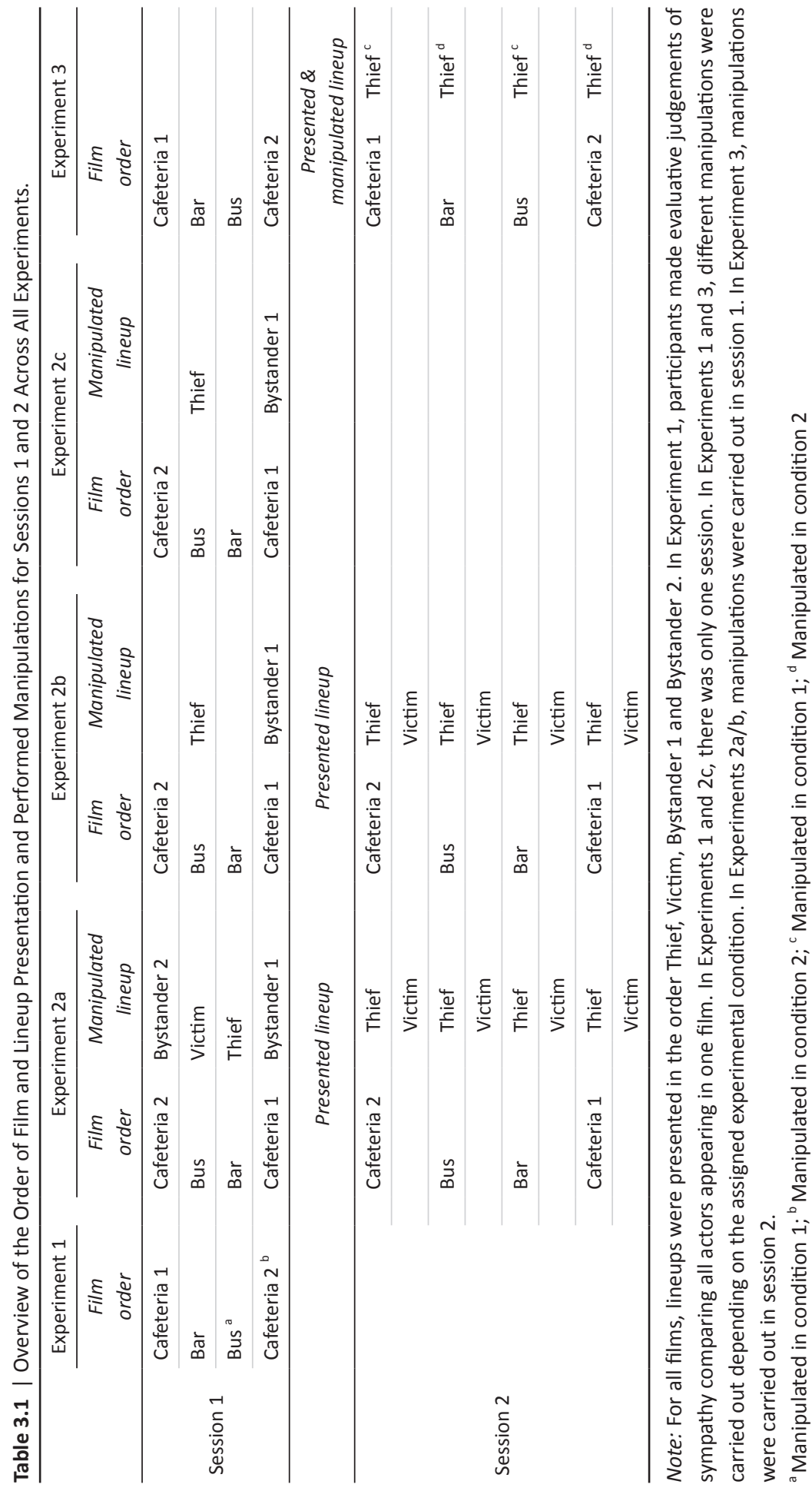




\section{Procedure}

The experimental procedure involved two sessions for Experiments $2 a / b$, but only one session for Experiment 2c. In session 1, participants, after signing the informed consent form, were told that they would watch four video fragments and act as eyewitnesses. Then, the first video was presented. Participants were asked to identify all four targets (i.e., perpetrator, victim, and two bystanders) involved in separate simultaneous photo lineups immediately after the end of the first video. Participants were forced to make a choice. Participants selected a lineup member by pressing one of the numbers indicated underneath each of the pictures in the lineup. Participants had unlimited time to make their choice. After a decision was made, a mask was presented for either $50 \mathrm{~ms}$ (Experiment 2a) or $500 \mathrm{~ms}$ (Experiment 2b; see Table 3.2). Subsequently, the photo of the selected lineup member was displayed on the screen and participants were asked to motivate their choice on a paper sheet. Once all four identifications were completed, the next video was presented. The same procedure was followed for all videos and targets. However, for four or two of the 16 lineups, participants were confronted with a photo they had actually not selected. Specifically, participants were presented with the photo presented dextrorotatory second next to the original choice regardless the accuracy of the identification. For example, if participants selected number 1 , they were presented with number 3 and if they selected number 6 , number 2 was presented. The exact order of the video fragments, the lineup order presentation, and the manipulations can be found in Table 3.1.

Session 2 (Experiments 2a/b) took place $24 \mathrm{hr}$ later. The aim of session 2 was to investigate whether the manipulations would have an impact on participants' future decisions. To that end, we asked the participants to identify all perpetrators and victims again (eight lineups in total). The cover story informed participants that we were interested in the effects of different lineup presentation methods. Accordingly, the lineup photos in session 2 were arranged in a single row rather than in a $2 \times 3$ matrix (as was the case in session 1 ). To avoid that participants reiterated the number they had chosen in session 1, the order of the lineup members also differed between the two sessions. Given that only the perpetrators and the victims were presented in session 2, there were only two previously manipulated lineups that carried over to the second session for Experiment 2a and only one for Experiment $2 b$ (see Table 3.1). This was done to avoid confusion and cognitive overload in the participants. Because peripheral targets are associated with reduced identification accuracy (Brown, 2003; Wessel, van der Kooy, \& Merckelbach, 2000), we decided not to present any bystanders in session 2. To make sure that participants were aware of which video fragment pertained to each lineup, they were presented with a sketch and a brief description of the plot before each lineup was shown. After completion of all eight identifica- 
tions, participants were given the post-test questionnaire. Finally, participants were thanked for their participation and fully debriefed.

\section{Results and Discussion}

\section{Blindness for Facial Recognition Decisions}

Concurrent detection across Experiments 2a-c varied between $65.0 \%$ and $68.9 \%$. For retrospective detection, percentages ranged between $94.4 \%$ and $100 \%$. Thus, overall more than $30 \%$ of the participants were concurrently blind, whereas retrospective blindness was largely absent. Note that we did not ask participants in Experiments $2 \mathrm{a} / \mathrm{b}$ how many manipulations they had noticed. Thus, we were unable to determine how many participants detected only some of the manipulations and how many detected all manipulations. For Experiment 2c, concurrent detection for the first manipulation was at $80.0 \%(n=16)$ and at $50.0 \%(n=10)$ for the second one. Retrospective detection rate was for both manipulations $100 \%(N=20)$. Table 3.2 summarizes the overall concurrent and retrospective detection rates across all Experiments.

Table 3.2 | Overview of the Experimental Design and Overall Percentage of Concurrent and Retrospective Detection across all Experiments.

\begin{tabular}{|c|c|c|c|c|c|c|c|}
\hline & \multirow{2}{*}{$\begin{array}{l}\text { Type of } \\
\text { Judgement }\end{array}$} & \multirow{2}{*}{$\begin{array}{l}\text { Performed } \\
\text { Choices }\end{array}$} & \multirow{2}{*}{$\begin{array}{c}\text { Performed } \\
\text { Manipulations }\end{array}$} & \multirow{2}{*}{$\begin{array}{l}\text { Retention } \\
\text { Interval }\end{array}$} & \multirow{2}{*}{ Sample } & \multicolumn{2}{|c|}{ Overall detection rate (\%) } \\
\hline & & & & & & Concurrent & Retrospective \\
\hline Experiment 1 & Evaluative & 4 & 1 & $50 \mathrm{~ms}$ & Students & 73.5 & 79.4 \\
\hline Experiment $2 \mathrm{a}$ & Absolute & 16 & 4 & $50 \mathrm{~ms}$ & Students & 66.7 & 94.4 \\
\hline Experiment $2 b$ & Absolute & 16 & 2 & $500 \mathrm{~ms}$ & Students & 68.4 & 100 \\
\hline Experiment $2 \mathrm{c}$ & Absolute & 16 & 2 & $500 \mathrm{~ms}$ & Non-academics & 65.0 & 100 \\
\hline Experiment 3 & Absolute & 16 & 2 & $48 \mathrm{hrs}$ & Students & 31.7 & 60.6 \\
\hline
\end{tabular}

Recognition Accuracy and Identification Shifts across Sessions (Experiments 2a/b)

To explore the possibility that our findings were mainly due to memory strength attributable to the attention that participants paid to the stimulus videos, we examined the overall recognition accuracy in the two sessions. In Experiment 2a, recognition accuracy across all targets was high, with an average of $M=76.9 \%(S D=13.3)$ in session 1 (across 16 targets) and an average of $M=90.8 \%(S D=12.3)$ in session 2 (across eight targets). In Experiment 2b, accuracy across targets was somewhat lower, with an average of $M=71.0 \%(S D=13.7)$ in session 1 and $M=84.0 \%$ (SD = 14.5) in session 2 . These high rates are not surprising, given that participants had to make a choice (forced choice) and that all lineups were presented in the target-present mode (Steblay, 1997). The higher accuracy rate for session 2 can be explained by the exclusive presentation of perpetrator and victim lineups in that session, whereas session 1 
also involved lineups for bystanders. Note that past research has indicated significant differences in the identification performance regarding central (i.e., perpetrators) vs. peripheral (i.e., bystanders) targets (Brown, 2003).

Furthermore, we tested whether the manipulation caused identification shifts from session 1 (i.e., before the manipulation) to session 2 (i.e., after the manipulation). For Experiment 2a, the average recognition accuracy for the two later-to-be manipulated lineups for both sessions was $M=86.1 \%(S D=23.0)$. For Experiment $2 b$, recognition accuracy was $M=100 \%$ for both sessions. Did participants alter their original choice at session 2 as a result of the manipulation? For Experiment 2a, only two participants changed their identification decisions. However, none of the observed decision changes were consistent with the manipulated outcome. In Experiment $2 b$, there were no switches in identification decisions. Apparently, our manipulations did not affect participants' identification performance in session 2.

In Experiments 2a-c, a significant proportion of participants concurrently failed to detect the manipulation, however, we found next to no retrospective blindness. Thus, in contrast to what previous studies (Johansson et al., 2008; Chapter 4; Sagana et al., 2013; Sauerland, Schell, et al., 2013) have found, blindness for eyewitness recognition decisions was limited to a concurrent level in Experiments 2a-c. Across the three experiments, we tried to rule out different explanations for the absence of retrospective blindness. In Experiment 2b, we showed that absence of retrospective blindness was not due to a relatively high proportion of manipulations or short mask duration that could have made our participants suspicious. Furthermore, the findings of Experiment $2 c$ argue against the idea that the absence of retrospective choice blindness in Experiments $2 \mathrm{a}$ and $2 \mathrm{~b}$ was due to sample characteristics such as prior knowledge, heightened alertness or both. Thus, when the manipulated outcome is presented immediately after a positive identification, and as long as a measure of retrospective detection is provided, blindness may not pose a serious threat for facial recognition decisions. Note, however, that a post-test questionnaire is normally not part of an eyewitness identification procedure.

Some may argue the absence of choice blindness in retrospective eyewitness judgments might be an artefact of the suggestive nature of the post-test questionnaire. However, this does not explain the vast differences in retrospective blindness found in our studies and previous choice blindness studies (e.g., Johansson et al., 2005; Chapter 4; Sagana et al., 2013; Sauerland, Schell, et al., 2013). Thus, in principal, the absence of choice blindness in the current experiments is good news as it suggests considerable preservation of the recognition decisions. Based on these results we would be happy to accept that choice blindness plays no (or little) role in this domain. However, it is unlikely that the identification procedure and the confrontation with one's decision at trial take place at the same day. In fact, even in so-called "speedy 
trial acts" as applied by the U.S. judicial system, trials usually begin at least 90 to 120 days after the arrest of the suspect (Shermer et al., 2011). Therefore, we wondered what the extent of blindness would be for recognition decisions that were taken in the recent past. Will witnesses notice the identity change if they are confronted with the manipulated outcome not milliseconds (i.e., short term memory) but hours or days later? Given the findings of Experiments 2a-c, we should not expect blindness. Conversely, Sauerland, Schell et al. (2013) revealed that people can be blind for changes in decisions related to long term autobiographical memory. Therefore, there may be a different cognitive process involved in long-term memory that could facilitate choice blindness.

To test these assumptions we conducted a third Experiment. In Experiment 3, we introduced a $48 \mathrm{hrs}$ interval between choosing a lineup member and being confronted with that very decision. After all, a delayed confrontation is more ecologically valid than an immediate one.

\section{EXPERIMENT 3}

\section{Method}

Experiment 3 employed the same video fragments and lineups as in the previous Experiments. Likewise, the procedure was analogous to Experiment $2 \mathrm{a}$ with the exception that the manipulated outcome was presented $48 \mathrm{hrs}$ after the recognition task.

\section{Participants}

Fifty-two (15 males, 37 females) Maastricht University undergraduates and one university employee $\left(M_{\text {age }}=20.8, S D_{\text {age }}=3.1\right.$, age range: $\left.17-35\right)$ took part in the study. Student participants had the following backgrounds: Psychology (86.5\%), Mental Health (9.6\%), and Law (1.9\%). All participants were tested individually and received either course credit or a small monetary award in return for their participation. Participants were naïve to the purpose of the study.

\section{Materials}

Post-Test Questionnaire. Participants were given an adjusted version of the post-test questionnaire employed in our previous Experiments. As in the previous Experiments, participants were first asked whether they had noticed anything strange during the Experiment and if they responded affirmatively, they were invited to provide details. If participants provided details from which we could infer that they had noticed a manipulation, they were coded as retrospective detectors. In contrast to the previous 
Experiments, participants were next (misleadingly) informed that the present study employed two experimental conditions, one in which some of the choices were manipulated (as in the actual Experiment), and one control condition where this was not the case. Participants then had to indicate in which condition they believed they had been assigned to. If a participant reported that $s /$ he believed to be in the manipulated condition, s/he also had to indicate how many times $\mathrm{s} /$ he had noticed a manipulation (Hall et al., 2010; Johansson et al., 2008).

\section{Procedure}

The Experiment involved two sessions and a follow-up. In session 1, participants watched first the video fragments and were then asked to identify all four actors from separate photo lineups, always in the order perpetrator, victim, and two bystanders. Participants were not given the option to reject the lineup. The same procedure was followed for all the remaining video fragments.

The second session was held 48 hrs later. In this session, participants were instructed to imagine that they were in a court room, with the victim and suspect present. The judge would now ask them to explain why they believed that the person they had chosen from the perpetrator lineup (i.e., the suspect) in session 1 was actually the perpetrator. The photo of the victim was presented to the participants before they were shown the picture of the suspect in order to provide a context and to avoid confusion between the four video fragments. Participants wrote down their reasons on a sheet of paper. If participants' statements indicated that they had noticed the change, these were coded as concurrent detection. The same procedure was followed for all four video fragments. However, for two of the four suspects, participants' choices were manipulated (see Table 3.1). That is, they were presented with a predetermined lineup member different from the one they had chosen two days earlier. Once participants finished this task for all four videos, they were asked to fill in the post-test questionnaire. Upon the completion of session 2 participants were thanked and fully debriefed.

To examine whether the manipulations would affect later decisions, we collected follow-up data. We hypothesized that participants would tend to choose the same lineup member that they had been shown during the manipulation. Three to five months after the original recognition task had taken place (i.e., session 1) participants were contacted via e-mail and provided with a PowerPoint file that included all four perpetrator lineups. The lineup photos were presented in two rows of 3 pictures, but in different order than at session 1 so as to avoid that participant selection would be based on their memory of the lineup number rather than memory of the identified face. Participants were asked to identify the four perpetrators again. Recall that two of these four lineups had been manipulated in session 2 . 


\section{Design}

In a $2 \times 2$ between-subjects design, we varied which of the four perpetrator lineups were manipulated (Cafeteria1 \& Bar vs. Cafeteria2 \& Bus), as well as the order in which the video fragments were presented (Cafeteria1-Bus-Bar-Cafeteria2 vs. Cafeteria2Bar-Bus-Cafeteria1). Participants were randomly assigned to the different conditions. Because the level of blindness for one's own recognition decisions $\left(\chi^{2} s(1) \leq 1.23\right.$, ps $\geq .40$, phis $\leq 0.15$ ), and recognition accuracy for manipulated or non-manipulated lineups $\left(\chi^{2} s(1) \leq 8.90, p s \geq .17\right.$, phis $\left.\leq 0.45\right)$ did not differ as a function of these two factors, we will not discuss them further.

\section{Results}

An alpha level of .05 was used for all analyses. For comparisons of means, we report Cohen's $d$ and $f$ (Cohen, 1988) as measures of effect size. For $2 \times 2$ and for $3 \times 2$ contingency tables, we report phi and Cramer's $V$ as measures of effect size, respectively.

\section{Blindness for Facial Recognition Decisions}

From the total of $2 * 52=104$ manipulated lineups, 33 (31.7\%) were detected concurrently, and $63(60.6 \%)$ retrospectively. Thus, in $39.4 \%$ of the cases participants were blind to the manipulations. Specifically, the first manipulation was concurrently detected by $23.1 \%(n=12)$ of the participants, the second one by $40.3 \%(n=21)$. Retrospective detection rates were $50.0 \%(n=26)$ and $71.2 \%(n=37)$, respectively. Hence, $28.8 \%(n=15)$ of the participants detected one manipulated lineup concurrently, $17.3 \%(n=9)$ detected both, and $53.8 \%(n=27)$ were blind for the two manipulations. Furthermore, $40.4 \%(n=21)$ of the participants detected one manipulated lineup retrospectively, $40.4 \%(n=21)$ detected both, and $19.2 \%(n=10)$ were blind for both manipulated lineups.

The blindness rates for the two manipulations suggest that the detection rate of the second manipulated lineup is inflated. This is understandable because participants probably become suspicious after the detection of the first manipulation (Johansson et al., 2005). To correct for this cascading effect, we discarded all second manipulated lineup detections when the first manipulation had been detected. Ninety-five manipulations remained for analyses. Of these, 24 (25.3\%) were detected. This distribution differed significantly from the expected, Cochran's $Q(1)=24.0, p<.001$. Subsequently, we ran all analyses for all 104 manipulations as well as for the corrected 95 that excluded cascading effects. By and large, the results for the 95 manipulated lineups were similar to those for the 104 lineups. Therefore, we will report the results for the full 104 manipulations. 


\section{Recognition Accuracy}

In Experiment 3, recognition decisions were not required for session 2. Analogous to our earlier analyses, we compared recognition accuracy of the two later to-bemanipulated perpetrator lineups only with the remaining two non-manipulated perpetrator lineups, given accuracy differences depending on the role of the target (e.g., perpetrators vs. bystanders; Brown, 2003). Accordingly, for the later to-bemanipulated lineups $(M=87.5 \%, S D=21.8)$ and for non-manipulated ones $(M=$ $91.4 \%, S D=19.1$ ) accuracies did not differ, $t(51)=0.94, p=.35, d=0.14$. Thus, there was no presumptive advantage for any of the lineup groups that could account for the choice blindness result.

Interestingly, participants who concurrently detected the first manipulation $(n=12)$ displayed greater recognition accuracy for the later-to-be-manipulated lineups $(M=$ $100 \%, S D=0.0)$ than those who were blind $(M=83.7 \%, S D=23.7), \chi^{2}(1, N=52)=$ $5.20, p=.02$, phi $=0.32$. Analogous results were obtained for the second manipulated lineup. Again, participants who concurrently detected the second manipulation displayed greater recognition accuracy for the later-to-be-manipulated lineups $(M=$ $95.2 \%, S D=15.0)$ than those who did not $(M=82.3 \%, S D=24.3), \chi^{2}(1, N=52)=4.50$, $p=.05$, phi $=0.29$. No such effects were found for retrospective detection, $\chi^{2} s(1, N=$ $52) \leq 9.23$, $p s \geq .52$, phis $\leq 0.13$. Furthermore, recognition accuracy for the later-to-bemanipulated lineups differed as a function of the number of detected manipulations (0, 1 or 2 detections), $\chi^{2}(2, N=52)=7.13, p=.03$, Cramer's $V=0.37$. Post hoc $2 \times 2$ chi-square tests revealed that participants who detected both manipulations concurrently $(n=9)$ were significantly more likely to be accurate $(M=100 \%, S D=0.0)$ than participants who were blind to both manipulations $(M=80.4 \%, S D=24.9), \chi^{2}(1, N=$ $37)=3.33, p=.02$, phi $=0.36$. Participants who detected one manipulation did not differ significantly $(M=93.3 \%, S D=17.6)$ from either of the other two groups, $\chi^{2} s(1) \leq$ $2.01, p s \geq .08$, phis $\leq 0.27$. No such effect was found for retrospective detection, $\chi^{2}(2$, $N=52)=1.61, p=.45, p h i=0.18$. Thus, concurrent detection seemed to be positively related to the memory strength of the original representation.

\section{Recognition Accuracy at Follow-Up}

From our $N=52$ participants, only $n=14$ replied to our follow-up email. Two of them (14.3\%) had been blind to our manipulations. The overall recognition accuracy at follow-up was relatively low with $M=60.7 \%$ ( $S D=27.2$ ). For the manipulated lineups, recognition accuracy for decisions made prior to the manipulation (session 1 ) was $M=89.3 \%(S D=21.3)$ and for identifications made in the follow-up $M=39.3 \%$ (SD = 35.0). For the non-manipulated lineups, recognition accuracy rates at session 1 were $M=89.3 \%(S D=21.3)$ and $M=82.1 \%(S D=31.7)$ at follow-up. Because of the small number of responses, no reliable statistical analysis could be performed. 
Did the previous manipulations affect participants' new identification decision? From the $2 * 14=28$ manipulated lineups, the same person as the one presented at the manipulation was selected only once. For 18 (64.2\%) lineup decisions, participants changed their selected lineup member to a different random alternative, while for 9 lineups (32.1\%) follow-up participants stuck to their original choice. Inspection of the two blind participants' responses revealed that for none of the lineups, they had selected the same person as presented at the manipulation. Thus, by and large, participants did not tend to select the lineup member suggested by the manipulation, although the manipulation seems to have undermined their accuracy. This finding is consistent with Experiments $2 a$ and $2 b$, but it deviates from previous studies (Merckelbach et al., 2011a; Sauerland, Schell, et al., 2013) that showed that blind participants tend to accept the manipulative misinformation at follow-up testing.

\section{Comparison of Choice Blindness across Experiments}

Next, we compared the detection rates across Experiments. Since neither concurrent, nor retrospective detection differed across Experiments $2 a-c$, all $\chi^{2} s(1) \leq 0.55$, ps $\geq$ .29 , phis $\leq 0.10$, we collapsed the data (total $N=57$ ). Pairwise comparisons between Experiment 1 ( 25 detectors of $N=34$ ) and Experiments 2a-c (40 detectors of $N=57$ ) revealed no differences in concurrent detection, $\chi^{2}(1, N=91)=0.01, p=.92$, phi $=$ 0.03 . However, a significantly lower level of retrospective detection in Experiment 1 (27 detectors of $N=34$ ) compared with Experiments 2a-c (56 detectors of $N=57$ ) emerged, $\chi^{2}(1, N=91)=5.74, p=.01$, phi $=0.30$. These results partially support our hypothesis that an evaluative component is responsible for increased blindness levels. Furthermore, there was a significantly lower level of concurrent detection in Experiment 3 (23 detectors of $N=52$ ), compared with Experiment 1 (27 detectors of $N=34$ ) and Experiments 2a-c (40 detectors of $N=57$ ), all $\chi^{2} s(1, N=91-109) \geq 6.02$, $p s \leq .01$, phis $\geq 0.26$. Retrospectively, there was a significantly lower level of detection for Experiment 3 (42 detectors out of $N=52$ ) compared with Experiments 2a-c (56 detectors of $N=57), \chi^{2}(1, N=109)=7.33, p=.01$, phi $=0.29$, but not compared to Experiment 1 (27 detectors of $N=34$ ). In line with our hypothesis, a longer interval between the identification task and the presentation of the manipulated choice led to increased levels of blindness. Table 3.2 summarizes concurrent and retrospective detection rates for all Experiments.

\section{Discussion}

The results of Experiment 3 indicate that eyewitnesses' blindness for facial recognition decisions is a non-trivial phenomenon. Specifically, 39\% (41) of all 104 manipulations performed in Experiment 3 remained undetected. The effect becomes even more striking when inspecting the concurrent blindness rate of the first manipulation. 
Indeed, $76 \%$ of the participants failed to notice, or at least failed to report, the change concurrently. To put this in perspective, one should consider that in practice, eyewitnesses are initially presented with a lineup. If a positive identification is made, the suspect is often arrested and incarcerated until the trial. In most European countries, the regulation of pre-trial detention applies, meaning that the identified person can be kept incarcerated till the trial without actual proof of guilt (Detained without trial: Fair Trials International's response to the European Commissions Green Paper on detention, 2011). Thus, even if the eyewitness, after the original identification at police headquarters, detects the change retrospectively in court, the innocent suspect would have spent days if not months in prison. Thus, our findings demonstrate the relevance of the choice blindness for this setting.

\section{GENERAL DISCUSSION}

It was the aim of the presented research to explore the conditions under which blindness for facial recognition decisions would occur. To this end, we conducted five Experiments. Specifically, we tested whether requiring evaluative in contrast to absolute decisions would induce blindness (Experiment 1). Our hypothesis that in an eyewitness setting, choice blindness rates would depend on decision type was only partially supported. Although there were no more concurrently blind participants in the evaluative than in the absolute decisions condition, absolute decisions yielded lower retrospective blindness rates. Furthermore, choice blindness levels for evaluative decisions in the eyewitness setting were low compared with other studies where participants performed evaluative judgements of visual stimuli (Johansson et al., 2005, 2008). In Experiments 2a-c, we employed simple recognition procedures and found moderate concurrent choice blindness rates and virtually no retrospective choice blindness in student and non-student samples. To increase the ecological validity of our findings, we inserted a $48 \mathrm{hrs}$ interval between the presentation of the recognition task and the presentation of the manipulated outcome in Experiment 3. Under these conditions, participants were concurrently blind for $68.3 \%$ and retrospectively blind for $39.4 \%$ of the manipulated lineups (see Table 3.2). Moreover, there was a tendency of participants who failed to notice the manipulation concurrently to be less accurate in session 1 than detectors. Blind participants' memory traces of the targets were apparently less strong that those of detectors, a fact that might have hindered them in detecting manipulations. Similar findings have been reported for blind recognition decisions in a field study (Chapter 4; Sagana et al., 2013). Nevertheless, in both studies the lower recognition accuracy for detectors compared to blind 
participants was absent for retrospective blindness rates, ruling out the possibility of an absolute memory advantage of detectors over blind participants.

Regarding our follow-up measure, the accuracy findings in our studies are compatible with previous studies that report an overall decrease in choice consistency for manipulated trials when participants are asked to perform their choices a second time (Johansson, Hall, \& Chater, 2012). For example, Sauerland, Schell et al. (2013, Experiment 2) reported a general tendency of follow-up participants to adjust their responses for the manipulated items so as to match the direction (e.g., positive vs. negative) of the manipulation. In our studies, recognition accuracy was not affected by the manipulation with short intervals (i.e., $24 \mathrm{hrs}$; Experiments 2a/b). For longer intervals, recognition accuracy was diminished when a decision had been manipulated (3-5 months follow-up of Experiment 3), although our participants did not switch to the lineup member presented during the manipulation. One might argue that accuracy simply degraded as a result of normal memory trace decline. However, if that interpretation was correct, the same pattern of results should have occurred for the non-manipulated lineups. This was not the case. Given the small number of responders in the follow-up, however, our findings should be read with appropriate caution.

We speculated that task ambiguity may explain why retrospective choice blindness was observed in Experiments 1 and 3, but did not occur in Experiments 2a-c. Ambiguity refers to vagueness and imprecision in the environment that allows for multiple interpretations (Sloman, Fernbach, \& Hagmayer, 2010). In a study on witness statements, stimulus ambiguity was found to reduce accuracy and to increase the chances of reporting false memories (Klimesch, Schimke, \& Pfurtscheller, 1993). Likewise, in Experiment 1, participants may have changed their sympathy evaluations. Given the absence of ground truth, such evaluations are inherently ambiguous. The identification decisions in Experiments 2a-c, however, did not require an evaluative judgement. Thus, the ambiguity inherent to evaluative tasks (Experiment 1) was absent by using more straightforward absolute (i.e., true/false) decisions (Experiments 2a-c). That ambiguity may help to set the stage for choice blindness is underlined by the higher levels of retrospective choice blindness in Experiment 1 than in Experiments 2a-c. Long retention intervals between the identification decision and the manipulated outcome would seem to be another way to introduce ambiguity. The findings of Experiment 3 support this line of reasoning. Additionally, in their field study, Sagana et al. (Chapter $4 ; 2013$ ) attributed the relatively high blindness rates to the real-life interaction which increased the complexity of the situation. Thus, task complexity may be yet another way to introduce ambiguity. All things considered, ambiguity seems to be a moderator for the occurrence of choice blindness.

The tendency of participants who failed to notice the manipulation concurrently to be less accurate in their recognition decisions than detectors (see also Chapter 
4; Sagana et al., 2013), could breed the belief that choice blindness in eyewitness settings is just a manifestation of memory degradation for the chosen alternative. In the introduction, we already provided reasons why memory decline is not sufficient to fully account for choice blindness. Note that recognition accuracy for blind participants was $82.3 \%$. Additionally, participants were willing to provide reasons not only for a non-selected alternative but also for an incorrect one. Taken together, our findings suggest that the interplay between task ambiguity and memory of the original trace might contribute to the effect.

At a theoretical level, blindness for facial recognition decisions seems a broad construct that integrates phenomena such as misinformation (Loftus, 2005; Loftus \& Hoffman, 1989) and post-identification feedback (Wells \& Bradfield, 1998). It is important, though, to make a distinction between the effects of a manipulation and those of blindness (i.e., acceptance of manipulation) in choice blindness procedures. Effects mainly driven by the presentation of the manipulated outcome and that are not dependent on the acceptance of the manipulation should be considered as consequences of the manipulation. Such effects were illustrated by Sagana et al. (Chapter 4 ; 2013) in terms of diminished rates of post-decision confidence in both blind participants and detectors. In the present study, the descriptively lower accuracy rates for the manipulated compared with the non-manipulated lineups in the follow-up of Experiment 3 might similarly reflect consequences of the manipulation. On the other hand, effects related to the internalization of the manipulation should be considered as consequences of blindness. This might be equivalent to the misinformation effect. Thus, the presentation of the manipulated outcome could lead participants to develop detailed recollection of choosing the manipulated outcome. Evidence for this internalization comes from the typical finding in choice blindness procedures that participants not only fail to detect the manipulation of their preferred outcome, but also confabulate reasons for their non-choices (Hall et al., 2010; Johansson et al., 2005). As testing the precise precursors of choice blindness is beyond the scope of the present article, our findings do not allow for disentangling the factors responsible for choice blindness. Our findings only provide the existence proof of choice blindness in an eyewitness context. However, given the aforementioned analogies, we believe that future studies on choice blindness and eyewitness identification might profit from an explicit consideration of the misinformation literature.

Apart from extending choice blindness to the domain of eyewitness recognition decisions, our study has important implications for legal settings. A situation in which an original eyewitness identification is being swapped with a different outcome may not be that unrealistic, as the case of Bernard Maughan (Wolchover, n.d.) exemplifies. A similar case came to our attention when the second author, while presenting parts of this research at a conference, was approached by a person from the audience 
who had been confronted with a change in her identification decision. Specifically, in court, the identified suspect was seated in the audience, while the suspect's brother was standing trial. Luckily, the witness was not blind to the error. Thus, errors in the recording of eyewitness decisions do occur. Indeed, an increasing body of research is concerned with the impact of biases due to contextual influences in the forensic science (e.g., Kassin et al., 2013; Saks \& Koehler, 2005). Additionally, recent findings suggest that lineup administrators' knowledge about the suspect's position in the lineup affects the evaluation of the lineup outcome in accordance to the expectancies of the administrator (Rodriguez \& Berry, 2014). Our point is that the types of manipulations we describe in the present article do occur in real-life. Our results furthermore show that it would be naïve to think that all eyewitnesses will be able to detect and correct such manipulations at a later stage.

Looking at the limitations of the present experiments, one obvious point is that our studies deviate from real-life procedures. Participants were confronted with the recognition task immediately after witnessing the mock crime, while the time between the crime and identification of the suspect usually varies from days to weeks (Behrman \& Davey, 2001). Likewise, the time between making an identification decision and the trial can be relatively long (Charman \& Wells, 2007). Nonetheless, if blindness for one's facial recognition decision is evident even when only days have passed; it is hard to believe that it would be absent when months or years have passed. An additional limitation is that the video fragments we used depicted rather minor crimes. If participants had seen a crime that would have been more emotionally provocative, then the pattern of results might have been different due to deeper processing of central features of the crime scene (Buchanan, 2007; LaBar \& Cabeza, 2006). Furthermore, if participants had seen the crime in a more ecological valid setting (i.e., live interaction), they might have been more resistant to choice blindness manipulations (Chapter 4; Sagana et al., 2013). On the other hand, we speculate, that due to shorter exposure times and numerous distracters inherent to real-life interaction, more ambiguity and therefore more blindness eyewitnesses would occur. Again, these issues warrant further studies.

Another limitation is that we only used target-present lineups and participants were forced to choose one of the lineup members. We realize that this procedure makes generalizations to standard lineup situations less obvious. Clearly, eyewitnesses in real cases are (or at least should be) warned that the perpetrator may or may not be present and they are given the option to reject a lineup (Malpass \& Devine, 1981; Wells et al., 1998). Under these conditions, many eyewitnesses decide to reject the lineup (i.e., nonchoosers; Horry, Memon, Wright, \& Milne, 2012). In the present studies, we opted against rejections as we were mainly interested in examining whether and to what extent choice blindness occurs for positive recognition decisions. In future 
research, it would be interesting to examine whether blindness occurs for identifications made from target absent lineups and for other lineup decisions (i.e., rejections). We hypothesize that nonchoosers would be more resistant to manipulations than choosers as the level of comparison would not be about the specific facial characteristic of the identified suspect ("Did I identify person A or person B?") but rather about a more broad decision outcome ("Did I or did I not make an identification?"). Thus, we expect that nonchoosers exhibit lower choice blindness levels than both accurate and inaccurate choosers, but this requires empirical testing.

To conclude, the current Experiments do not only replicate previous findings showing that choice blindness is relevant for eyewitness identification decisions, but also advance our understanding of the circumstances that may be decisive for the occurrence of the effect. Foremost, our findings point at ambiguity as a key factor for the occurrence of choice blindness. Furthermore, we believe that our findings bear relevance to research on eyewitness identification decisions. Specifically, the choice blindness paradigm has the potential to become a postdictor of eyewitnesses' accuracy. However, more research is warranted to establish whether being a detector safely denotes higher odds of accurate identification. Finally, our findings have important implications for the judicial system. We believe that research on choice blindness will add to our knowledge about the decision making errors of eyewitnesses. 


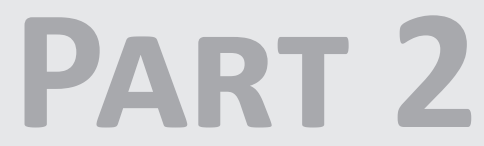

TESTING BLINDNESS IN THE FIELD 



\section{4}

Witnesses' blindness for their own facial recognition decisions: A field study 


\section{ABSTRACT}

In a field study, we examined choice blindness for eyewitnesses' facial recognition decisions. Seventy-one pedestrians were engaged in a conversation by two experimenters who pretended to be tourists in the centre of a European city. After a short interval, pedestrians were asked to identify the two experimenters from separate simultaneous six-person photo lineups. Following each of the two forced-choice recognition decisions, they were confronted with their selection and asked to motivate their decision. However, for one of the recognition decisions, the chosen lineup member was exchanged with a previously unidentified member. Blindness for this identity manipulation occurred at the rate of $40.8 \%$. Furthermore, the blindness rate varied as a function of similarity (high vs. low) between the original choice and the manipulated outcome. Finally, choice manipulations undermined the confidence-accuracy relation for detectors to a greater degree than for blind participants. Stimulus ambiguity is discussed as a moderator of choice blindness. 


\section{INTRODUCTION}

People are confronted with hundreds of decisions daily. Some are of minor significance, whereas others have the power to cause profound changes to one's life. Yet, experiments demonstrate that people are sometimes poor in monitoring their decision outcomes if their choices are manipulated. Johansson, Hall, Sikström, and Olsson (2005) dubbed this effect choice blindness. In their first demonstration of choice blindness, participants were asked to select the more attractive alternative from pairs of female faces. Subsequently, they were given the selected face and were asked to explain the reasons underlying their choice. On three of the 15 trials, however, participants were presented with the opposite of the chosen picture. Surprisingly, the overwhelming majority (87\%) of the manipulated trials went undetected right after the manipulation (i.e., concurrently).

Choice blindness has been documented with visual (Johansson et al., 2008), auditory (Chapter 2; Sauerland, Sagana, et al., 2013), gustatory, and olfactory stimuli (Hall et al., 2010), and across different ages groups (Chapter 8; Sauerland, Sagana, Otgaar, \& Broers, 2014). Furthermore, stimulus similarity has been shown to temper the effect (Hall et al., 2010; Chapter 2; Sauerland, Sagana, et al., 2013), although other studies have found no such effect (Johansson et al., 2005; Chapter 8; Sauerland et al., 2014).

Despite the apparent robustness of choice blindness in the laboratory, one might wonder whether the effect would occur when poor monitoring of choices would have serious consequences. To address this question, Sauerland, Schell, et al. (2013) examined blindness for one's own history of norm-violating behaviours. Participants were asked to indicate the frequency with which they had displayed certain transgressive behaviours in the past on a four-point Likert scale (i.e., never, seldom, sometimes, often). After either 10 minutes or a one week delay, participants were interviewed about four of these items. However, their answers to two target items had been increased or decreased by two full scale points. Of the sixty-five participants, $25 \%$ were blind to at least one of the two manipulations after a 10 minutes delay. This percentage increased to $45 \%$ with the one week interval. These findings suggest that choice blindness is not - solely - memory based. Indeed, it is quite unlikely that participants had forgotten how many times they had committed certain violations. Yet, the fact that the longer interval led to increased blindness implies that forgetting one's own retrieval (i.e., forgetting the indicated choice) plays a role, although this cannot account for the effect per se. Importantly, the study shows that choice blindness also occurs with stimulus material that is of personal significance.

Apart from memory failure and stimulus similarity, ambiguity has been proposed as a moderating factor for choice blindness (Merckelbach et al., 2011a). Accordingly, choice blindness occurs whenever decisions pertain to subjective experiences. Evalu- 
ative judgements, such as facial attractiveness (Johansson et al., 2008; Johansson et al., 2005) or food preferences (Hall et al., 2010), are accompanied by an inherent ambiguity. This is thought to facilitate choice blindness. Following this line of reasoning, one would predict that an objective decision that lacks ambiguity, as for example an identification decision, would make target manipulations much easier to detect.

This notion was recently examined by Sagana, et al. (Chapter 3; 2014b), who had their participants watch a series of mock crime video fragments and then identify the targets from simultaneous target present (TP) lineups. Following their forced-choice identification, participants were confronted with their selection and were asked to provide reasons for their decision. Two of the 16 identifications were manipulated, leaving participants with a different non-chosen lineup member. In line with the ambiguity (and memory trace decline) explanation, the retrospective detection rate was high (94-100\%) when a confrontation with the manipulated outcome occurred shortly after the identification (Experiments 1a, 1b, 2). However, when a 48hrs interval between the identification and the confrontation with the manipulated outcome was inserted (Experiment 4), the retrospective detection rate deflated to $61 \%$. Accordingly and similar to Sauerland, Schell et al.'s (2013) study, when memory became fuzzier due to longer intervals, participants were more likely to be blind to the manipulation. In other words, the ambiguity of the identification task due to time lag eliminated the positive impact of a clearly defined (i.e., non-ambiguous) decision. Taken together, these findings support the ambiguity explanation as a moderator of choice blindness (Merckelbach et al., 2011a).

Given the parallels of choice blindness with suggestibility for misleading information (for reviews, see Ayers \& Reder, 1998; Loftus, 2005), one could argue that the present line of research actually portrays different manifestations of the same effect. In a typical demonstration of misinformation, upon presentation of the misleading information witnesses' memory trace is contaminated, thus lowering the accuracy of the reports for a witnessed event. Likewise, in choice blindness, the presentation of the manipulated outcome serves as the suggestive information that impairs the memory of the original target. However, for choice blindness, memory strength does not seem to be the cause, but rather one of the various moderators of the effect. Thus, choice blindness seems to be a broader construct that incorporates suggestions and misleading information.

With these considerations in mind, we aimed to investigate whether blindness for one's eyewitness identifications emerges for real life identification decisions in a field study. This would allow us to determine whether the effect is prominent in the context of eyewitness identifications outside the laboratory. We hypothesised that the short duration and the various distracters inherent to real life interactions would introduce ambiguity to the task, resulting in high levels of blindness despite immedi- 
ate confrontation with the manipulated items. Furthermore, we wanted to test again whether similarity between the originally identified suspect and the manipulated outcome would affect blindness levels. We expected high similarity between targets and manipulated stimuli to introduce an additional source of ambiguity, resulting in increased levels of blindness. Similarly to Sagana et al.'s (Chapter 3; 2014b) study, only TP lineups were used and participants were not given the option to reject the lineup (forced choice). We realize that this procedure makes generalizations to standard lineup situations less obvious. We nevertheless opted for it as we were, at this stage, mainly interested in determining whether the effect would arise in real life situations.

\section{METHOD}

\section{Participants}

Seventy-one pedestrians (32 men, 39 women) participated in the study $\left(M_{\text {age }}=\right.$ 36.2 years, $S D_{\text {age }}=15.4$, age range: $\left.18-64\right)$. Participants worked in the private sector (16.9\%), public sector (16.9\%), and academia (8.5\%), were students (26.2\%), were retired: $(4.2 \%)$, or worked in other professions (27.3\%). Participation was voluntary and no incentives were granted. The study was approved by the university's standing ethical board.

\section{Materials}

\section{Lineups}

A total of four TP photo lineups were created. Target persons were four female undergraduate psychology students aged 21-24 years who participated as part of a bachelor course. The lineups consisted of six $8.7 \times 7.6 \mathrm{~cm}$ frontal face photos, presented in two rows à three pictures on a $26.0 \times 20.0 \mathrm{~cm}$ display board. All foils fit the general descriptions of each of the targets, as determined by a pilot study with $N=32$ mock witnesses (effective sizes, determined as Tredoux's Es were between 4.92 and 5.75; Tredoux, 1998; Tredoux, 1999).

\section{Similarity}

To test the similarity between the originally identified photo and the photos presented as manipulations, a pilot study $(N=26)$ was performed. In this study, all members of each lineup were paired with each other and rated with regard to their similarity on a seven-point Likert scale ( 1 = not similar at all, $7=$ highly similar). The least and the most similar picture for each lineup member was selected to be presented as the manipulated outcome, creating the two similarity conditions (each containing 24 
pairs). The averaged means for the low and the high similarity pairs was $M=4.11,95 \%$ $\mathrm{Cl}[3.9,4.3]$ vs. $M=2.98,95 \% \mathrm{Cl}[2.8,3.2]$, respectively, $t(23)=10.19, p<.001, d=2.1$.

\section{Post-Test Questionnaire}

To examine whether participants had noticed our manipulation and refrained from revealing it, we administered a post-test questionnaire (adjusted from Johansson et al., 2008). First, participants were asked if they had any remarks, if they had encountered any problems, and if yes what the nature of these problems was. Then, participants were misinformed that the current study employed two conditions: one in which their choice was manipulated and one where this was not the case. Participants had to indicate to which condition they thought they had been assigned. If participants said that they belonged to the former condition, they were asked to indicate how many times and which lineup they had noticed to be manipulated. These answers were counted as retrospective detection.

\section{Design}

Similarity (low vs. high) between the target picture and the picture presented as manipulation was varied in a one factorial between-subjects design. Detection rates were measured concurrently and retrospectively. Concurrent detection refers to detection immediately after the presentation of the manipulated photo. Retrospective detection refers to detection during the post-test questionnaire.

\section{Procedure}

Data were collected in groups of three, two persons acting as targets and one as Experimenter. In total, four different students acted as targets and three as Experimenters. The combination of targets was counterbalanced to avoid recognition effects caused by differences in distinctiveness resulting in six different target combinations. All but one Experimenter (who gathered data of three participants) collected data of four participants with each target pair. Since the level of blindness did not differ as a function of the Experimenter (all $\chi^{2} s(2) \leq 2.67, p s \geq .263$, Cramer's $V s \geq 1.94$ ) or as a result of the different target combinations (all $|t| s(69) \leq .123, p s \geq .902, d s \geq 0.09$ ), we will not discuss these factors any further.

Data collection took place in the centre of a western European city. Specifically, two targets pretending to be tourists approached a random pedestrian asking for directions. The targets had predetermined roles mainly to help the Experimenter to later specify for which of the targets the first and the second identification was referring to. The primary target would be the first one to talk to the pedestrian and to lead the conversation. The secondary target interacted at a lower level with the pedestrian and was responsible for keeping track of the time. The conversation was scheduled 
to last between 30 to 60 seconds. Then, the two targets walked into the direction the pedestrian had indicated. Each target acted both as primary and as secondary target. Identification accuracy (defined by hits) did not differ as a function of target, $F(3,70)$ $=0.95, p=.422, f=.20$.

The Experimenter watched the situation from a distance. Around 40 seconds after the conversation had come to an end $s /$ he approached the pedestrian and explained that the two young women asking for directions were actually students conducting a study on eyewitness identification. If consent to participate was given, the Experimenter presented the lineup of the primary target. Participants were given unlimited time to make a decision, but were not allowed to reject the lineup. Subsequently, they rated how confident they felt about their identification decision on an 11-point scale ranging from $0 \%$ to $100 \%$. The same procedure was followed for the secondary target. Participants were then asked to write down a short description of the event. This was done to introduce a short interval before participants were confronted with their identifications. Finally, participants were presented with the photo of the person they had identified in the primary and the secondary lineup and were sequentially asked to motivate their decisions. However, the identification made for the secondary target was always manipulated, leading participants to end up with a different photo than the one they had selected. The manipulated photo was either of high or of low similarity with the original choice, depending on the condition the participant was assigned to. Subsequently, participants were again asked to provide a confidence estimate for their earlier identification. Due to a procedural slip (neglect to present the scale) though, confidence data after the manipulation were unavailable for the first 19 participants, leaving 52 participants for these confidence analyses. Participants were then handed the post-test questionnaire. Upon completion participants were thanked and fully debriefed.

\section{RESULTS}

An alpha level of .05 was used for all inferential analyses. For comparisons of means, we report Cohen's $d$ and $f$ (Cohen, 1988) as measures of effect size. For $2 \times 2$ and for 2 $\times 3$ contingency tables, phi and Cramer's $V$ are reported, respectively.

\section{Blindness for One's Identification Decisions}

Overall concurrent detection was $31.4 \%(n=22)$ and retrospective detection was $59.2 \%(n=42)$. Thus, in total $40.8 \%(n=29)$ of the participants were completely blind to our manipulation. This percentage is in line with previous laboratory research into blindness for identification decisions (Chapter 3; Sagana et al., 2014b). 
Concurrent detection varied as a function of similarity, $\chi^{2}(1, N=71)=9.98, p=.002$, phi $=0.38$. As expected, participants in the low similarity condition were more likely to concurrently detect the manipulation (48.6\%), compared with participants in the high similarity condition (13.9\%). Likewise, for retrospective detection, participants in the low similarity condition were more likely to detect the manipulation (74.3\%) than participants in the high similarity condition $(44.4 \%), \chi^{2}(1, N=71)=6.54, p=.016$, phi $=0.30$.

\section{Identification Accuracy, Post-decision Confidence and CA Relation}

Across the two targets, participants displayed low levels of identification accuracy $(M=46.4 \%, S D=39.0)$, perhaps due to the life setting and the short exposure to the stimuli. Furthermore, participants' performance was more accurate for non-manipulated $(57.7 \%)$ than for manipulated lineups $(35.2 \%), \chi^{2}(1, N=71)=5.27, p=.026$, phi $=$ 0.27 . This is curious given that the targets in manipulated and non-manipulated trials were the same ones. Note, however, that manipulations were always performed for the secondary, more passive target. Given the less intense interaction with this target, compared to the primary target, the observed difference in identification accuracy was actually to be expected. Similar findings have been reported earlier, with lower identification accuracy in TP lineups for an accomplice than a perpetrator (Dempsey \& Pozzulo, 2008). Wells and Pozzulo (2006), however, reported higher accuracy for the accomplice than the perpetrator, although they acknowledge that their finding is atypical.

We were most interested in whether blind participants differed from participants who detected the manipulation (detectors) in identification accuracy. Concurrent detectors were not more accurate than blind participants for the manipulated lineup, $\chi^{2}(1, N=71)=1.46, p=.226$, phi $=0.14$. An effect was found, however, for retrospective detectors who were more accurate $(45.2 \%)$ than blind participants $(20.7 \%), x^{2}(1$, $N=71)=4.53, p=.044$, phi $=0.25$. No such differences were observed for the nonmanipulated lineups, all $\chi^{2} s(1, N=71) \leq .133, p s \geq .715$, phis $\leq 0.04$.

As to post-identification confidence, participants in general displayed low levels of confidence before $(M=57.5 \%, S D=21.8)$ and after the manipulation $(M=48.7 \%, S D=$ 20.6). Interestingly, for the later to-be-manipulated lineup, retrospectively blind participants were less confident $(M=43.1 \%)$ than retrospective detectors $(M=59.5 \%)$, $t(59.7)=2.80, p=.007, d=1.78$. For concurrent detection, the effect did not attain significance, $t(69)=1.83, p=.071, d=1.11$, although inspection of the means suggested somewhat lower confidence for blind participants $(M=60.8 \%)$ than detectors $(M=65.5 \%)$. For the non-manipulated lineup, no differences occurred, all $\mid t / s(69) \leq$ $1.35, p s \geq .180, d s \leq 0.77$. 
In a next step, we conducted two mixed Analyses of Variance (ANOVA) with concurrent and retrospective detection (detectors vs. blind participants) as between-subjects variables and manipulation status (manipulated vs. non-manipulated lineups) and test time (before vs. after the manipulation) as within-subjects factors. We expected that this method would provide a more in depth analysis of the interplay between manipulation and blindness and confidence. Note that only $n=52$ participants were included for this analysis. The results for concurrent and retrospective detection were analogous. The three way interaction effect between detection, manipulation status, and test time was significant, for concurrent, $F(1,51)=16.26, p<.001, f=$ .33 and retrospective detection, $F(1,51)=5.47, p=.023, f=.35$. Consequently, we performed separate ANOVAs for detectors and blind participants, with manipulation status and test time as within-subject factors. For concurrently and retrospectively blind participants, there were significant main effects of manipulation status on postdecision confidence, $F(1,35)=16.83, p<.001, d=0.57 ; F(1,19)=10.77, p=.004, d=$ 0.62 . Confidence rates were lower when a lineup had been manipulated $(M=44.7 \%$, $S D=20.1 ; M=37.5 \%, S D=24.1)$ relative to when it had not been manipulated $(M$ $=58.2 \%, S D=24.7 ; M=53.5 \%, S D=27.1)$. All other effects were non-significant for blind participants, all $F s(1,35) \leq .799$, $p s \geq .396$, $d s \leq 0.04$. For detectors, there was a significant interaction of manipulation status and test time for concurrent, $F(1,15)$ $=9.70, p=.007, f=.43$, and retrospective detection, $F(1,32)=9.14, p=.005, f=$ .65. Analyses of the simple main effects revealed that for the manipulated lineups, confidence estimates given before the manipulation were higher $(M=55.0 \%, S D=$ 26.3; $M=55.3 \%, S D=23.1)$ than estimates provided after the manipulation $(M=$ $21.9 \%, S D=29.9 ; M=36.9 \%, S D=28.7)$ for concurrent, $F(1,15)=11.09, p=.005, d$ $=1.17$, and retrospective detection, $F(1,31)=9.36, p=.005, d=0.71$. No such effect was found for the non-manipulated lineups for concurrent, $F(1,15)<0.01, p=1.00, d$ $<0.01$, or retrospective detection, $F(1,31)=0.04, p=.845, d=0.04$.

Finally, we examined whether the confidence-accuracy (CA) relationship was different for blind participants vs. detectors. Note that a point-biserial correlation of $r_{p b}=$ .37 is considered large, $r_{p b}=.24$ moderate, and $r_{p b}=.10$ small (Cohen, 1988). Prior to the manipulation, the effect size of the CA association for detectors was small for the later to-be-manipulated lineup, $r(40)=.10, p=.553$, but large for the non-manipulated lineup, $r(40)=.42, p=.005$. For blind participants, the effect was large for both the later to-be-manipulated, $r(27)=.54, p=.003$, and the non-manipulated lineup, $r(27)$ $=.48, p=.009$ (see Table 4.1 for a summary of the obtained CA correlations).

For detectors, confidence measured after the manipulation was negatively associated with identification performance for the manipulated lineup, $r(30)=-.23, p=$ .208. For the non-manipulated lineup, however, the CA correlation was positive and large, $r(30)=.36, p=.040$. For blind participants, the effects were large for both the 
manipulated lineup, $r(18)=.40, p=.083$, and the non-manipulated lineup, $r(18)=.53$, $p=.015$. Note, however that lack of power prevented the former correlation from reaching significance. Overall, the results indicate that our manipulations had a more detrimental effect on the CA relation for detectors than for blind participants.

Table 4.1 | Point-Biserial Confidence-Accuracy Correlations for Manipulated and Non-Manipulated Lineups.

\begin{tabular}{lcc}
\hline & Manipulated lineup & Non-manipulated lineup \\
\cline { 2 - 3 } & $r_{p b}$ & $r_{p b}$ \\
\hline Before Manipulation & & $.42^{* *}$ \\
\hline Detectors $(n=42)$ & .10 & $.48^{* *}$ \\
\hline Blind $(n=29)$ & $.54^{* *}$ & \\
\hline After Manipulation & & $.36^{*}$ \\
\hline Detectors $(n=32)$ & -.23 & $.53^{*}$ \\
\hline Blind $(n=20)$ & .40 & \\
\hline
\end{tabular}

Note. Due to a procedural error, confidence data after the manipulation were unavailable for 19 participants, leaving 52 participants for these analyses. ${ }^{*} p<.05 . * * p<.01$.

\section{DISCUSSION}

The current study examined blindness for one's eyewitness identification decisions in a real life setting. A subsidiary aim was to assess the role of similarity between the original choice and the manipulated outcome as a moderator of the effect. As expected, a large portion of participants failed to notice our manipulation concurrently (68.6\%) and retrospectively (40.8\%). Furthermore, blindness was facilitated by high similarity between target and manipulated outcome.

Replicating earlier findings (Chapter 3; Sagana et al., 2014b) the current study shows that blind identification decisions can occur at an alarmingly high level. Importantly, the effect was found for a broad age range and under realistic encoding conditions, enhancing the ecological validity of our findings. Nevertheless, there are some important differences between the present field study and real world conditions. Specifically, due to ethical considerations, participants did not witness a real crime. Furthermore, they were not allowed to reject the lineup. Also, the intervals between the witnessed event, the identification decision, and the confrontation with the manipulated outcome were much shorter than in a real life case. Yet, the testing conditions were less artificial than those in the typical laboratory experiment. Thus, although we might not be able to draw direct parallels to real world lineup identifications, the present study suggests, at a minimum, that blindness for one's identification decisions may be more prominent in real life than in the laboratory. Specifically, in Sagana et al.'s (Chapter 3; 
2014b; Experiments 2a-c) laboratory studies concurrent detection rates ranged from $66.7 \%$ to $75 \%$, while retrospective detection varied from $94.4 \%$ to $100 \%$ when the manipulated outcome was presented immediately after the identification. In the current field study, the concurrent detection rate was $31.4 \%$ and retrospective detection was $59.2 \%$. These relatively low detection rates might be attributable to the turbulence and dynamics of the real life setting. Note that when Sagana et al. (Chapter 3; Sagana et al., 2014b; Experiment 3) inserted a 48hrs interval between the identification and the presentation of the manipulated outcome, the concurrent detection deflated to $31.7 \%$, retrospective detection to $60.6 \%$, coming very close to the rates obtained in the present study. This convergence speaks to the validity of laboratory simulations and underlines the importance of conducting field research.

Our findings also support the hypothesis that when an element of ambiguity (i.e., everyday circumstances, longer interval) is introduced to the identification task, blindness for one's identification decisions becomes more prominent. Alternatively, one could argue that the high blindness rates found in the current study result from the low identification accuracy rates for the manipulated lineup. Indeed, retrospective detectors showed higher identification accuracy than blind participants, although this was not observed for concurrent detectors. This finding implies that blindness for one's identification decisions depends on the original memory trace for the target. That is, witnesses with poor memory of the target fail to detect the discrepancies between the original representation and the manipulated outcome. However, earlier studies demonstrated that choice blindness is not a purely memory-based effect (Sauerland, Schell, et al., 2013). Furthermore, the current detection rates are comparable to prior findings (Chapter 3; Sagana et al., 2014b), where identification accuracy was over $87 \%$. Our identification accuracy rates are also in accordance with a recent field study (Horry et al., 2012) that summarized data of over 1,000 real lineups administered in England. Only 39.0\% involved a suspect identification. It is unclear, though, how many of these lineups actually included the perpetrator.

Conversely, the low accuracy - high blindness effect fits well with the ambiguity explanation for choice blindness (Merckelbach et al., 2011a). The poor memory for the original target may have caused an increased feeling of ambiguity in our participants while performing the identification task, hence fostering blindness. The ambiguity hypothesis is further supported by the findings regarding the similarity of the chosen and the presented photo in manipulated lineups. The high similarity between these photos may have worked as an additional source of ambiguity, leading to increased blindness rates for that condition, compared with the low similarity condition.

As for post-decision confidence, blind participants were less confident than detectors for the later to-be-manipulated lineup. This suggests that low confidence may facilitate blindness. Note that the overall low confidence and accuracy rates for both 
targets may reflect the difficulty of the identification task. Therefore, it may be the increased ambiguity inherent to the difficulty of the task that caused more blindness. Another explanation is that participants who wanted to reject the lineup in the first place indicated very low confidence, hence deflating the average confidence rates. Unfortunately, with the current design, we can only speculate about the role of confidence as a moderator of blindness. Future studies relying on TA and TP lineups in fully counter-balanced designs could shed more light on this role.

Also related to post-decision confidence is the finding that our manipulation affected its relationship with identification accuracy. Specifically, the manipulation undermined post-decision confidence. However, blind participants, in contrast to detectors, displayed strong CA relations both before and after the manipulation. The more prominent effect for detectors compared with blind participants implies differences in the decision making of the two groups. A plausible explanation for this pattern could be that detectors might feel confused about the target for which they were to provide confidence ratings. Other studies have also found long-lasting effects of the manipulations studies. Specifically, in a follow-up, participants had to make a choice for the same stimuli as in the initial first round. The results showed that participants either chose the alternative that had been presented to them in the manipulated trial (Johansson et al., 2011) or adjusted their responses to match the direction of the manipulation (e.g., positive vs. negative, Merckelbach et al., 2011a; Sauerland, Schell, et al., 2013). Curiously, Sagana et al. (Chapter 3; Sagana et al., 2014b; Experiment 3) reported that when participants performed the same identification task three to five months after the initial one, participants' new, incorrect choices did not accord with the manipulated foil.

There are several limitations to our study that warrant discussion. Firstly, because we relied exclusively on TP lineups and forced choice decisions, the generalizability of our findings to real world investigations is limited. In real lineup procedures, participants would (or at least should) be warned that the perpetrator may or may not be present and be given the option to reject the lineup (Wells et al., 1998). A second limitation, which is related to the forced choice decisions, concerns the low confidence identifications generated in our study. In real cases, it is unlikely that such low confidence identifications would be accepted as evidence in court. Thirdly, no criminal act was staged. We cannot exclude the possibility that high stress levels of an emotionally intense event would have affected the blindness rates. On the one hand, stress could impair the memory of the target (Deffenbacher, Bornstein, Penrod, \& McGorty, 2004) leading to increased ambiguity when identifying the target and thus more blindness. On the other hand, stress could facilitate encoding of the witnessed event and facilitate memory for the target (Christianson, 1992) leading participants to be less willing to accept the manipulation. Fourthly, only a short retention interval 
was inserted between the event and the identification task. In real cases, intervals would generally be longer (Horry et al., 2012; Shermer et al., 2011). Yet, a longer interval would cause the memory trace to fade, producing more ambiguity and probably higher rates of blindness (e.g., Sagana et al., 2012). Finally, we did not determine whether the manipulation had a long-lasting effect. This is difficult to implement in a field study. Nevertheless, such results could provide valuable insight regarding the impact of choice blindness on meta-cognition, long after the manipulation took place.

Moving to the practical relevance of the study, our findings have important implications for lineup administrators and law enforcement bodies. Indeed, a lineup administrator could mistakenly write down the wrong identification decision. Given the present findings, one should expect that a large proportion of eyewitness would fail to detect such a mistake. One may object that the records of identification decisions are unlikely to be incorrect or that witnesses usually sign their names next to the photo of the identified suspect. Considering, though, how often professionals make procedural errors, we suspect that such scenario is not all that unrealistic. As a matter of fact, errors made by forensic scientists such as mistakes in testing procedures (erroneous matches of bullets, foot- and fingerprints) together with police misconduct, are a major source of miscarriages of justice (Saks \& Koehler, 2005). Having said that, it is worth mentioning that the second author, while presenting our research findings at a conference, was approached by a person from the audience who had experienced a switch in the identification she had made. Specifically, the person she had identified was present in the audience in court, while his brother was standing trial. The witness noticed this, but what if she had not? It might be worthwhile to explore systematically whether there exist similar procedural errors and how they were handled.

Apart from the lineup identifications, the effect of choice blindness can be relevant for identifications pertaining to events that took place many years before they are investigated, such as war crimes. Here, eyewitnesses often have to compare their memory of the perpetrator with a photo of that person from the distant past and a suspect of an elder age (e.g., John Demjanjuk's case; Loftus \& Ketcham, 1991). The increased ambiguity of the situation may facilitate blindness effects, as it is suggested to eyewitnesses that the suspect matches the memory of the perpetrator, using the presentation of the photo as proof.

Finally, our findings may inspire new research on eyewitnesses' reports. Specifically, it is known from previous studies that people are relatively poor at recognising fabricated details in reports of one's own autobiographical memories (Barclay \& Wellman, 1986; Merckelbach, Wessel, \& Horselenberg, 1997). In the present study, we found that eyewitnesses are poor at monitoring the outcome of their identification decisions minutes after their choice. Hence, it seems reasonable to assume that witnesses would fail to detect surreptitious changes in their description of a witnessed 
Chapter 4

crime due to choice blindness. This issue warrants further study and more generally, it would be interesting to explore how typical misinformation effects and choice blindness are related to each other (e.g., whether choice blindness sets the stage for misinformation effects). 


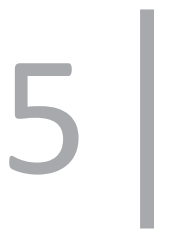

The effect of choice reversals on blindness for identification decisions 


\section{ABSTRACT}

The present study examined blindness for identification decisions from targetpresent and target-absent lineups using a field study methodology. Eighty pedestrians were exposed to a staged theft. Subsequently, they were asked to identify the thief and the victim from separate, simultaneous six-person lineups. Their identification decision concerning the thief lineup was manipulated such that participants' selections were exchanged with a previously unidentified lineup member (choice exchange) and lineup rejections were turned into identifications (choice reversal). Participants were seven to ten times less likely to detect choice exchanges (66.7\%) compared with choice reversals (11.2\%). Furthermore, identification accuracy was not a prerequisite for detection. Thus, rejections and particularly selections made from both target-present and target-absent lineups are susceptible to choice blindness. Finally, our study implies that for blindness in eyewitness identification decisions between-category changes (i.e., choice reversals) are easier to detect than within-category changes (i.e., choice exchanges). 


\section{INTRODUCTION}

At first glance, the case of B. Maughan (Wolchover, n.d.) looks like a typical police lineup procedure: A witness identified lineup member six from a live lineup in the presence of a solicitor. Subsequently, the lineup administrator wrote down and read back the identification statement to the witness and B. Maughan was charged with involvement in a road rage attack. What the lawyer who reviewed the identification tapes on appeal found, though, took him by surprise. The lineup administrator had written down a different lineup member than the one identified by the witness. Although the administrator repeated aloud the wrong lineup number, both the witness and the solicitor failed to detect the mistake. This, then, signals a real live instance of an altered lineup decision that remained unnoticed (for details see Chapter 3; Sagana et al., 2014b). Although anecdotal, the Maughan case raises the question to what extent and under what conditions the outcome of eyewitness identification decisions can be corrupted by this type of error.

Recent research on the effect of choice blindness suggests that people can be oblivious to secret changes in the outcome of their decisions (Johansson et al., 2005). Applying the choice blindness paradigm in the case of lineup decision manipulations, Sagana, Sauerland and Merckelbach (Chapters 3 \& 4; 2013, 2014b) found that almost $40 \%$ of the witnesses failed to notice the change. Specifically, Sagana et al. (Chapter 4; 2013) engaged pedestrians in a conversation with two experimenters who pretended to be tourists. Subsequently, pedestrians had to identify these two tourists from simultaneous, six-person photo lineups, using a forced-choice recognition format. Having provided a description of the witnessed event, participants were presented with their lineup decisions and were asked to motivate them. However, their selection for the second target was exchanged with another previously non-selected lineup member. Most participants (69\%) failed to detect the identity manipulation when confronted with it (i.e., concurrent blindness). Furthermore, participants who had been accurate in their recognition decision from the outset were more likely to detect the manipulation than those who had been inaccurate. To address the possibility that participants had noticed the change but refrained from revealing it, participants were informed about the possibility of a manipulation at the end of the experiment. Even then the blindness rate was still 40\% (i.e., retrospective blindness). Moreover, earlier research suggests that compliance (Johansson et al., 2008; Johansson et al., 2005; Chapter 2; Sauerland, Sagana, et al., 2013), suggestibility (Merckelbach et al., 2011a; Sauerland, Schell, et al., 2013) or the tendency to react in socially desirable ways (Merckelbach et al., 2011a; Chapter 2; Sauerland, Sagana, et al., 2013) do not moderate blindness.

Additionally, Sagana, et al. (Chapter 3; 2014b) aimed to determine the border conditions of blindness for recognition decisions. In the controlled environment of 
the laboratory and when the manipulated outcome was presented immediately after the selection (Experiments 2a-c), blindness for recognition decisions was comparably low (concurrent: $25-33 \%$, retrospective: $0-6 \%$ ). When a $48 \mathrm{hr}$ interval between the recognition decision and the presentation of the manipulated outcome was inserted (Experiment 3), concurrent (68\%) and retrospective (39\%) blindness rates approached the levels reported in the field study. However, unlike the findings in the field study (Chapter 4; Sagana et al., 2013), identification accuracy did not predict neither concurrent nor retrospective detection rates. Thus, the authors suggested that memory strength, which is an important determinant of identification performance, is insufficient to explain blindness phenomena. Further support for this position comes from the finding that participants are capable of remembering their choices with precision when asked to do so, despite being blind to manipulations (Chapter 9; Sagana, Sauerland, \& Merckelbach, 2014a). Another finding to consider is that blindness phenomena can occur minutes after the original choice (Hall et al., 2010; Johansson et al., 2005) as well as for decisions of personal relevance (Chapter 9; Hall et al., 2012; Sauerland et al., 2014) and for autobiographical reports (Sauerland, Schell, et al., 2013), where one expects memory to be strong.

The work by Sagana et al. (Chapters 3 \& 4; 2013, 2014b) was an essential first step in showing that blindness may have far-reaching implications for the legal domain. As the ambition of these pioneer studies was to provide an existing proof of blindness in typical eyewitness settings, the complexity of the experimental designs was restricted to keep the impact of confounders at a minimum. Specifically, these studies exclusively relied on target-present lineups and a forced choice format. However, the innocence or guilt status of the suspect is unknown in real cases, and every administered lineup may potentially be a target-absent lineup. In a target-absent lineup a covert manipulation, where foil identification would be turned into a suspect identification, could lead to the imprisonment of an innocent suspect on false premises. Furthermore, in real cases eyewitnesses should be warned that the perpetrator may or may not be present in the lineup (Wells et al., 1998) and witnesses should be given the option to reject the lineup (i.e., nonchoosers). Accordingly, the question arises whether turning a lineup rejection into a positive identification (i.e., choice reversal) would elicit lower blindness rates than an exchange of lineup members (i.e., choice exchange). In the present study, we addressed this issue by examining blindness phenomena under conditions that are comparable to real lineup identification decisions.

Employing a field study methodology similar to the one used by Sagana et al. (Chapter 4; 2013), the current study aims to replicate previous findings on blindness for identification decisions and to expand our understanding of these errors by a) presenting target-absent and target-present lineups and b) providing the option to reject the lineup. This enabled us to manipulate both choosers' and nonchoosers' decisions. 
Specifically, nonchoosers' rejections were turned into identifications (i.e. reversal) and choosers' identification decisions were exchanged (i.e., exchange; swapped with a different lineup member). We refrained from turning lineup identifications into rejections because they are of little relevance to the legal system, as there is no incentive for this type of conversion (although we acknowledge the possibility of an honest mistake). Finally, to further increase the ecological validity and the generalizability of our findings, a live mock crime was employed. All participants were individually exposed to the live theft of a backpack. Although a staged crime cannot mimic a real crime scene for ethical reasons, it can introduce the element of surprise and thus simulate realistic encoding conditions. Therefore, field experiments can differentiate our understanding of the mechanisms involved in eyewitness identification (Cutler \& Penrod, 1995). The present field experiment provides insight into the readiness of eyewitnesses to detect changes in their identifications, for an event that occurred under conditions of divided attention which resemble real life. We therefore consider the field methodology a valuable research approach.

We assume that the mismatch between participants' decision and the manipulated outcome would be greater for nonchoosers than for choosers. We base this assumption on the belief that a selection implies a match between the memory of the culprit and one of the lineup members, while a rejection reflects a reluctance to identify any of the faces in the lineup as the culprit (Sporer et al., 1995; Weber \& Brewer, 2006). Thus, when presented with the manipulated outcome, for choosers the comparison would rest on the specific facial characteristic of the identified suspect ("Did I identify this person or another person?"), while for nonchoosers the comparison would rest on a higher and less ambiguous decision level ("Did or did I not make an identification?"). In other words, the manipulation constitutes a within-category change for choosers but a between-category change for nonchoosers. Accordingly, we hypothesize that nonchoosers would be more resistant to manipulations than choosers. In line with this assumption is also research on the related phenomenon of change blindness, which refers to the inability to detect visual changes if these are combined with visual disturbance (e.g., eye-movement, blink, distracting stimuli). For example, presented with intact and scrambled versions of schematic faces, participants were more likely to detect a change when intact and scrambled faces were interchanged (betweencategory) than when a scrambled or intact face was changed into another scrambled or intact face (Lyyra, Mäkelä, Hietanen, \& Astikainen, 2014). These results indicate that between-category changes are more easily detected than within-category changes. Moreover, assuming that rejections occur more often for target-absent than 
for target-present lineups, we expect blindness rates to be higher for target-present compared with target-absent lineups. Finally, based on earlier findings suggesting that even people who are accurate in their identification decision are susceptible to blindness manipulations (Chapter 4; Sagana et al., 2013), we expect identification accuracy to be unrelated to blindness levels in both choosers and nonchoosers.

\section{METHOD}

\section{Participants}

Eighty participants (19 male, $M_{\text {age }}=21.6, S D_{\text {age }}=4.7$, age range $18-49$ ) who were mostly bachelor (58.9\%) and master (31.3\%) students on their way to enter a university building. took part in this study. The educational level of eight participants $(10.0 \%)$ is missing because, in order for the thief to remain visible equally long amongst participants, the play of the crime scene interfered with the collection of the demographics. All participants were tested individually on a voluntary basis and no incentives were granted. The study was approved by the standing ethical committee of the faculty.

\section{Material}

\section{Lineups}

Three target-absent (TA) and three target-present (TP) simultaneous photo lineups were created. The target persons were three psychology students (one female, age range: 22-25 years). Head and shoulder photos were selected to fit the description of the respective targets (effective sizes determined as Tredoux's $E$ values ranged from 4.13 to 5.00; Tredoux, 1998; 1999). Lineups consisted of six $7.0 \times 6.0 \mathrm{~cm}$ frontal face, head and shoulder photos, presented in three pictures a row, on a $29.7 \times 21.0 \mathrm{~cm}$ laminated display board. Each lineup included one target (i.e., perpetrator or victim) or replacement of the target and five foils. On the right side of the board, next to the six photos, a rejection option was provided.

\section{Post-test questionnaire}

In order to examine whether participants noticed our manipulation, yet refrained from mentioning it, we administered a post-test questionnaire (adjusted from Johansson et al., 2008). Participants were first asked whether they encountered any problems during the experiment and what the nature of these problems was. Subsequently, they were falsely informed that the study employed two conditions: one in which their identification decisions had been changed (experimental condition) and another where their decisions had remained intact (control condition). Participants were in- 
vited to indicate in which condition they thought they had participated. If participants responded that they had been assigned to the experimental condition, they had to indicate how many times and for which target(s) they had noticed a manipulation. These answers, if correct, were counted as retrospective detection.

\section{Design}

A 2 (manipulation status: manipulated vs. non-manipulated lineups) x 2 (lineup mode: TA vs. TP) within-subjects design was employed. To avoid recognition effects caused by differences in distinctiveness and anchoring, we counterbalanced a) which of the two male experimenters ${ }^{2}$ acted as the victim or the actual experimenter, and $b$ ) the sequence of TA and TP lineup mode across perpetrator and victim lineups (i.e., TA-TP, TA-TA, TP-TA, and TP-TP). Detection rates did not vary as a function of the experimenter, all $\chi^{2} s(1, N=80) \leq 1$. 93, ps $\geq .165$, phis $\leq .15$, or as result of the lineup mode sequence, all $\chi^{2} s(3, N=80) \leq 7.16, p s \geq .062$, Cramer $V s \leq .30$. Therefore we will not discuss these factors any further.

Detection rates served as the dependent variable and were measured concurrently and retrospectively. Concurrent detection refers to detection immediately after the presentation of the manipulated outcome (e.g., "I haven't chosen her. I believe the correct culprit in not among the photos", or "I chose No 2 because of her hair style."). Retrospective detection additionally includes detection reported in the post-test questionnaire. That is, all concurrent detections are per definition also retrospective detections. Finally, based on the identification outcome two additional dependent variables were calculated, namely lineup outcome (selection vs. rejection) and identification accuracy (accurate vs. inaccurate).

\section{Procedure}

Data collection took place at the campus of a European university. Participants were individually approached by one of the two male experimenters and were asked if they wanted to participate in a short study on face perception. This cover story was used to mask the real purpose of the study and to facilitate the controlled unfolding of a mock crime that took place while participants were engaged in a conversation with the experimenter. Upon agreement, participants signed a first informed consent form. At that point the experimenter set down his backpack slightly behind him a few centimetres away from his standing point. Subsequently, the experimenter engaged participants in a conversation about their demographics. Meanwhile, the 'thief' approached the scene. While navigating herself around the experimenter and the

2 A female experimenter with an Icelandic background acted always as the thief. This had to do with her limited capacity to interact with the participants in their native language (i.e., Dutch), which was a safety prerequisite imposed by the ethical committee. 
participant the thief kept track of the time on her cell phone so that she would be visible for 40 seconds. After 40 seconds had elapsed, she grabbed the experimenter's (who from this point onward will be referred to as the victim) backpack and rushed into the university building. After initially acting confused (to allow the thief to get out of sight), the victim excused himself and hurried after the thief.

At that instant, participants were approached by the second experimenter who immediately revealed that the event had been staged and explained that the study was about eyewitness testimony. If consent to continue with the study was given, the victim and thief lineups were presented in succession. Participants were instructed that the target may or may not be present, were allowed to reject the lineup, and were given unlimited time to make a decision. In order to introduce a short interval between the identification decision and the confrontation with this decision, participants were asked to provide a short written description of the event. Although no time limit was imposed for this task, participants took no more than a few minutes to complete their reports (note that the entire experiment lasted no more than 15 min). Next, participants were presented with their two lineup decisions (i.e., with the identified lineup member or a question mark when the lineup had been rejected) and were asked to explain the reasons for their decision. Participants were always first presented with their victim lineup decision and then with the thief lineup decision. However, the decision made for the thief lineup was manipulated. Specifically, lineup member 1 was exchange with lineup member 2, member 3 with member 4, and member 5 with member 6 . In the case of a lineup rejection, participants were presented with the photo of the perpetrator when they had been presented with a TP lineup earlier and the photo of the replacement of the target when they had been presented with a TA lineup. This was done because these manipulations are the only ones that could result in a conviction. We refrained from manipulating both lineups (victim and thief). Rather we first confronted participants with their decision in the non-manipulated lineup. This was to gain participants' trust and attention and to familiarize them with the lineup procedure (see Chapter 4; Sagana et al., 2013 for a similar approach). Furthermore, we manipulated the thief lineup decision, rather than the victim lineup decision, as this decision is forensically most relevant. Finally, participants received the post-test questionnaire, were thanked, and fully debriefed.

\section{RESULTS}

\section{Blindness for Identification Decisions}

Across all 80 performed manipulations, concurrent blindness was $68.7 \%(n=55)$. Retrospectively, $55.0 \%(n=44)$ of the manipulations remained undetected. As expected, 
participants were less likely to be blind to manipulations from TA (concurrent: $M=$ $55.0 \%, 95 \% \mathrm{Cl}[39.8,69.3]$; retrospective: $M=42.5 \%, 95 \% \mathrm{Cl}[28.5,58.7])$ than from TP lineups (concurrent: $M=82.5 \%, 95 \% \mathrm{Cl}[68.1,93.1]$; retrospective: $M=67.5 \%, 95 \%$ $\mathrm{Cl}[52.0,79.9])$, all $\chi^{2} s(1, N=80) \geq 5.05, p s \leq .025$, phis $\geq .25$, although lineup outcome (selection vs. rejection) did not depend on target presence, $\chi^{2}(1, N=80)=1.86, p=$ .172 , phi $=.15$.

\section{The Effect of the Non-manipulated Lineup on Blindness Rates}

Considering that the manipulated lineup was always presented second, we first tested whether the identification decision in the non-manipulated lineup affected blindness rates. Identification accuracy for the victim lineup was high $(M=72.5 \%, 95 \% \mathrm{Cl}[61.9$, 81.1]), and considerably lower for the thief ( $M=42.5 \%, 95 \% \mathrm{Cl}[32.3,53.4]), M c N e m a r$ $\chi^{2}(1, N=80)=12.02, p=.001$. The longer interaction time with the victim (i.e., the experimenter) compared with the thief may have been responsible for this difference, despite the central role of the thief in the crime scene. Consequently, we performed binary logistic regression analyses with detection (detected vs. non-detected) as dependent variable, and lineup outcome (selection vs. rejection) and identification accuracy (accurate vs. inaccurate) of the non-manipulated victim lineup as predictors. Neither of the factors predicted blindness rates, all Wald $\chi^{2} s(1, N=80) \leq 0.75$, ps $\geq$ .388. Therefore, for the remaining analyses we discuss result only for the thief lineup; that is the lineup where a manipulation was performed.

\section{Blindness as a Function of Lineup Outcome}

In the current study, we were most interested in whether lineup outcome could determine participants' ability to detect our manipulations. To this end, we performed

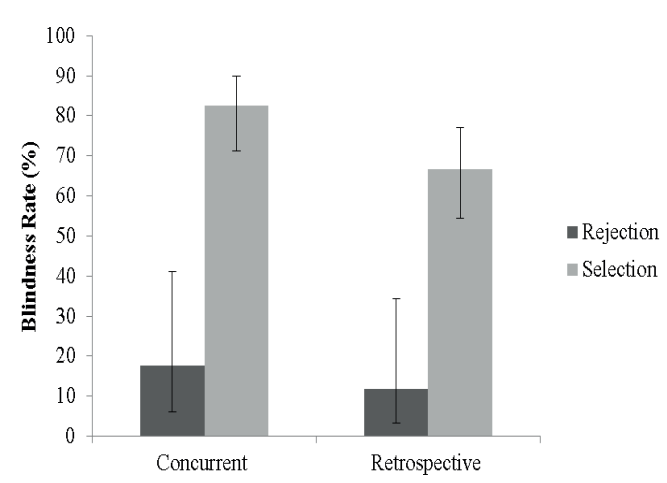

Figure 5.1 | Proportion of concurrent and retrospective detection as a function of lineup outcome (selection vs. rejection). Error bars represent $95 \%$ confidence intervals. 
binary logistic regression analyses with detection (detected vs. non-detected) as dependent variable, and lineup outcome (selection vs. rejection) as predictor. Analyses including target presence (present vs. absent) and the interaction effect of target presence and lineup outcome as additional predictors did not increase the variance explained by the model (concurrently: $82.5 \%$; retrospectively: $71.3 \%$ ). Therefore, we will only discuss the simpler model. The analysis returned a significant effect of lineup outcome for concurrent, Wald $\chi^{2}(1, N=80)=18.59, p<.001$, and retrospective detection, Wald $\chi^{2}(1, N=80)=11.49, p=.001$. Figure 5.1 represents the mean concurrent and retrospective blindness rates as a function of lineup outcome. Inspection of the associated odds ratio showed manipulations involving selections to be 10 times less likely to be detected concurrently and 7.5 times less likely to be detected retrospectively than manipulations involving rejections. Thus, as expected, lineup selections were associated with higher blindness rates.

One might argue that the difference in blindness rates for rejections vs. selections could be attributed to the fact that for selections we performed manipulations both towards and away from the suspect as these different kinds of manipulations are different in nature. To address this concern we performed the analysis again, this time including only those selections that involved a change towards the suspect. The results remained largely the same, with lineup outcome being a significant predictor of concurrent, Wald $\chi^{2}(1, N=31)=9.73, p=.002$, and retrospective detection, Wald $\chi^{2}(1, N=31)=6.18, p=.013$. Manipulations of selections were 5.4 to 9.0 times less likely to be detected than those of rejections.

\section{Blindness as a Function of Identification Accuracy}

Considering the vast differences in detection rates between selections and rejections, we examined the role of identification accuracy on detection separately for selections and rejections. The low number of blind participants $(n=3)$ among those who rejected the lineup $(n=17)$ allows only a descriptive approach to the data. At a descriptive level, of the 11 participants who made a correct rejection 10 (90.9\%) detected the change both concurrently and retrospectively. Of the 6 participants who made an incorrect rejection 4 (66.7\%) detected the change concurrently and 5 (83.3\%) did retrospectively. However, given the small number of the observations, we cannot draw conclusions about the effect of accuracy on blindness rates for lineup rejections. For selections, chi-square tests looking at detection rates as a function of identification accuracy revealed a significant effect of accuracy on concurrent, $\chi^{2}(1, N=63)=4.32, p$ $=.044$, phi $=.26$, but not on retrospective detection, $\chi^{2}(1, N=63)=2.19, p=.193$, phi $=.19$. Specifically, participants who made an accurate selection from the thief lineup displayed higher blindness rates concurrently $(M=93.7 \%, 95 \% \mathrm{Cl}[86.6,100])$ than those who made an erroneous selection $(M=75.0 \%, 95 \% \mathrm{Cl}[61.0,89.0])$. 


\section{DISCUSSION}

The present study aimed to investigate the effect of blindness for eyewitnesses' identification decisions under naturalistic encoding conditions. Above and beyond the replication of earlier findings indicating that blindness phenomena occur for eyewitness recognition decisions (Chapters 3 \& 4; Sagana et al., 2013, 2014b), the current study extended our knowledge by showing that blindness occurs under conditions that come close to a real eyewitness setting. Importantly, our findings suggest that between-category changes are easier to detect than within-category changes in eyewitness identification decisions.

In particular, participants remained concurrently blind to our covert manipulations at a $68.7 \%$ rate and retrospectively at a $55.0 \%$ rate. Furthermore, our hypothesis that manipulations applied to rejections would be more likely to be detected compared with manipulations applied to selections was supported by the findings. In fact, rejection manipulations were approximately seven to ten times more likely to be detected than selection manipulations. Relatedly, blindness rates for manipulations of targetabsent lineups were relatively low compared with those of target-present ones. Clearly, the effect may ally with the higher number of nonchoosers in target-absent than in target-present lineups (on a descriptive, but not statistically significant level). Finally, and in line with previous work looking into the role of memory in blindness phenomena (Chapters 3, 8 \& 9; Sagana et al., 2014a, 2014b; Sauerland et al., 2014), poor identification accuracy was not a precondition for blindness. In fact, when identification accuracy influenced blindness, the effect was in the opposite of the expected direction. Indeed, for selections, blind participants were more accurate than detectors. This is a surprising finding pointing to the fact that, before we can formulate firm conclusions about the effect of accuracy on blindness, more conceptual replications of the present study are needed.

Consequently, it may be difficult to attain blindness for lineup rejections that are turned into identifications. Nevertheless, the reduced probability of blindness for manipulations applied to rejections should not be confused with a lack thereof. A non-trivial minority (12-18\%) of participants did accept having made a positive identification while they had in fact rejected the lineup. Thus, our findings do not imply that a rejection per se inoculates against blindness but they signal that the obviousness of the change is critical for the effect. We would expect a selection that is turned into a rejection to elicit comparable detection rates as a rejection that is turned into a positive identification. To conclude, the probability of noticing changes applied to one's identification decision is higher for higher-order and thus more fundamental choice reversals compared with choice exchanges. Similarly, and despite the detection advantage of target-absent lineups, the high blindness rates for both target-absent and 
target-present lineups affirm that identification decision manipulations can affect the guilty as well as the innocent target.

From a theoretical stance, the increased detection rate for nonchoosers compared with choosers appears to be related to a greater attentional shift for the betweencategory than the within-category changes (Lyyra et al., 2014). This interpretation is consistent with the asymmetric features in choosers' and nonchoosers' identification decisions possess (Sauerland et al., 2012; Weber \& Brewer, 2004). Specifically, the eyewitness literature holds that while choosers base their identification decisions on the match between their memory of the culprit and one of the lineup members, nonchoosers' decisions seem to reflect a reluctance to identify any of the faces in the lineup as the culprit (Sporer et al., 1995; Weber \& Brewer, 2006). Therefore, when presented with a different lineup member, to detect changes choosers should focus their attention on the specific facial configuration of the manipulated face. Conversely, nonchoosers' attention should be more easily drawn to the fact that they are actually presented with a face while they should be presented with none. Hence, for nonchoosers the subsequent presentation of a selection constitutes a distinct category conversion that is difficult to adopt as it directly contradicts their decision. From a theoretical point of view, turning a selection into a rejection should be an alteration equally difficult to accommodate as turning a rejection into a selection. That is because once a choice has been made, the witness is under the impression that at least one lineup member was a match. Hence, both types of manipulations (i.e., a rejection turned into a selection and vice versa) would constitute a between-category change. Although in the present study, we refrained from turning selections into rejections, a study testing this type of reversal may be beneficial for the theoretical understanding of blindness for identification decisions.

An alternative, yet related explanation for the increased detection of choice reversals comes from research on blindness for manipulations of self-reported symptoms (Merckelbach et al., 2011a, 2011b). Specifically, Merckelbach et al. reported that participants who were blind to manipulations of their psychological symptom ratings had initially scored higher both on the manipulated and the control symptoms compared with detectors. This indicates that participants who more frequently gave zero responses reflecting categorical decisions ("I don't have this symptom") were more likely to detect manipulations of their symptom ratings. Drawing from the misinformation literature (Loftus, 2005; Loftus \& Hoffman, 1989), Merckelbach et al. attributed the stronger tendency to blindness for non-zero responses to an increased ambiguity participants experienced compared with giving zero responses. That is, participants who gave non-zero responses may be more ambivalent as to which answer they provided than participants who categorically rejected the symptom as a whole. This in turn raises the probability of misinformation acceptance. Our findings 
are in line with this conceptualization because a lineup rejection can be viewed as an equivalent of a zero response.

Moving to the practical relevance of the present study, our findings imply that an innocent suspect might be at risk when a smooth manipulation takes place in a real-world investigation. Notably, the risk is greater in the presence of foil identifications rather than rejections. Although some might object to the idea of identification decisions being altered in real investigations, the Northern California Innocence Project report on prosecutorial misconduct cases sides with the idea (NCIP; Ridolfi \& Possley, 2010). The report reveals 4,000 cases of alleged misconduct, in 707 of which the courts explicitly established that the prosecutors deliberately mishandled, mistreated or destroyed evidence. Against this background, our findings highlight the importance of double-blind identification administration that leaves little room for secret manipulations.

All in all, the current study extends the findings that blindness phenomena are relevant to eyewitness identifications by demonstrating that such phenomena can occur for choosers and, to a lesser extent, nonchoosers, as well as for target-present and, to a lesser extent, target-absent lineups. Hence, identification decision manipulations could jeopardize the liberty of innocent suspects. Furthermore, our findings suggest that it is more difficult to convert a lineup rejection into a positive identification than to swap among identifications, which is good news for the judicial system. In sum, then, our findings contain important lessons for lineup administrators and law enforcement bodies. Certainly, future research is to determine the magnitude of blindness in real cases. Having said that we believe that research on blindness for identification decision is critical for the legal practice as it can provide useful insights into eyewitnesses' decision making. 


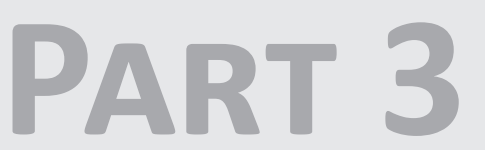

EXPANDING THE APPLICATION 



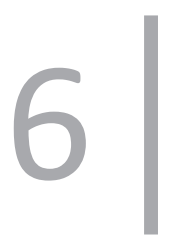

\section{Eyewitnesses' blindness for own- and other-race identification decisions}




\section{ABSTRACT}

The aim of the present study was to test the impact of own-race bias on eyewitnesses' blindness for their own identification decisions. We hypothesized that blindness for one's identification decision would increase for other-compared with own-race identifications due to the increased ambiguity of the former condition. To test this hypothesis, Black African $(n=40)$, White African ( $n=$ $43)$, and White European participants $(n=40)$ were asked to identify white targets. Specifically, participants watched four mock crime films and made 16 identifications from target-present and target-absent simultaneous lineups. All targets were of Caucasian origin. Forty-eight hours later, participants returned to the lab and were asked to justify four of their identification decisions. However, for two of the four identifications participants were not presented with their original choice, but with a different non-identified lineup member. In line with our hypothesis, participants who made other-race identifications were less likely to detect the manipulations (concurrent: $5 \%$, retrospective: $31 \%$ ) compared with participants who made own-race identifications (concurrent: 12 - 21\%, retrospective: $41-45 \%)$. Unexpectedly, rejections that were turned into identifications were not more likely to be detected than interchanged identification decisions. These findings shed light on how choice blindness may affect eyewitness identification decisions and highlight its potential negative consequences for legal proceedings. 


\section{INTRODUCTION}

The doctrine that we are rational thinkers, who deliberate and objectively weight evidence, is a deeply encrypted belief. But how much can we rely on the rationality of our decisions? Research on the so-called choice blindness shows that people often fail to detect changes in the outcome of their decisions, when these are secretly manipulated (Johansson et al., 2005). In a typical choice blindness experiment, participants are asked to indicate their preferred alternative in a binary task. In a subsequent interview, they are confronted with the non-chosen alternative, which is presented as it was their choice. The acceptance of this manipulated outcome as the product of their own choice is termed choice blindness. In fact, for choices concerning facial (Johansson et al., 2008; Johansson et al., 2005), auditory (Chapter 2; Sauerland, Sagana, et al., 2013), gustatory and olfactory decisions (Hall et al., 2010), no more than $13-19 \%$ of choice switches are detected immediately (concurrent detection). When participants are asked about the performed manipulations after the end of the experiment (retrospectively), detection rates are no higher than $44 \%$. Thus, people seem to have limited insight into their decision outcomes.

Recently, Sagana, et al. (Chapter 4; 2013) examined choice blindness for eyewitnesses' facial recognition decisions. Specifically, pedestrians in the centre of a European city were engaged in a conversation with two experimenters who pretended to be tourists. Shortly thereafter, the pedestrians were asked to identify the two experimenters from separate simultaneous photo lineups using a forced-choice recognition decision format. Subsequently, they were confronted with their selection and asked to motivate their decision. However, for the second target, the chosen lineup member was swapped with a previously non-identified member. Concurrently only $31.4 \%$ and retrospectively $59.2 \%$ of the participants detected this identity manipulation. These detection rates are elevated compared with previous choice blindness research. This should not come as surprise though given that an identification task requires an absolute judgment (correct vs. incorrect) compared with an evaluative judgment that preference decisions incorporate (Chapter 3; Sagana et al., 2014b). Hence, the authors argued that introducing an element of ambiguity to the task facilitates blindness (see also, Merckelbach et al., 2011a). Ambiguity refers to vagueness and imprecision in the stimuli that allow for multiple interpretations (Sloman et al., 2010). Ambiguity may occur in a variety of everyday circumstances such as long retention intervals between indicating the decision and being presented with the manipulated outcome, or bad viewing conditions. We assume that other factors that increase task ambiguity also facilitate blindness. 
One such factor with clear legal implications is to induce cross-race identifications. According to the own-race bias (ORB) people have difficulties in recognizing faces ${ }^{3}$ of a race other than their own (Barkowitz \& Brigham, 1982; Brigham \& Barkowitz, 1978; for a meta-analytic review see Meissner \& Brigham, 2001). In a classic ORB paradigm other-race identifications elicit a lower proportion of accurate identifications and a higher proportion of misidentifications than own-race identifications. Apparently, people store own-race faces more accurately and efficiently in memory compared with other-race faces, resulting in confusion and vagueness (e.g., Rhodes et al., 2009; Wright, Boyd, \& Tredoux, 2003).

Several theories aim to explain the recognition advantage for own compared with other race faces (for a review see Sporer, 2001b; Young et al., 2012). Following Young et al.'s (2012) classification, three broad theoretical perspectives emerge: perceptual expertise, social cognitive and hybrid theories. Perceptual expertise theories (e.g., differential processing style, Michel et al., 2007; Rhodes et al., 1989; face space model, Valentine, 2001) explain ORB as people's greater ability in processing and distinguishing own compared with other race faces. Conversely, social cognitive theories (e.g., feature selection model, Levin, 2000; cognitive disregard, Rodin, 1987) attribute ORB in peoples' tendency to think categorically (i.e., in-group vs. out-group). Hence, they rely on the broad social group membership instead of the unique characteristics of a face, predominantly for out-group targets. Lastly, the more recent hybrid theories (e.g., categorization-individuation model, Hugenberg, Young, Bernstein, \& Sacco, 2010; ingroup/outgroup model, Sporer, 2001a) postulate that both perceptual expertise and social cognitive factors contribute to the ORB. Regardless the mechanism though, all theoretical perspectives hold that identifications for other-race faces are more difficult compared with own-race ones, and thus more ambiguous. As said before, ambiguity appears to foster choice blindness. With these considerations in mind, the present study tested whether eyewitnesses' blindness would be more prominent for other than for own-race identification decisions.

To fully grasp the influence of the ORB on eyewitnesses' blindness for their identification decisions, it is important to examine the interracial contact hypothesis. This hypothesis holds that the ORB is a consequence of limited contact with other races (Brigham \& Malpass, 1985; Chiroro \& Valentine, 1995). A crucial prediction that can be derived from this hypothesis is that persons who have high interracial contact would be equally accurate in recognizing faces of their own as well as of the other race. To test this, Chiroro and Valentine (1995) compared Africans and Britons who had

3 In the context of eyewitness identification, ambiguity and task difficulty may appear equivalent. Indisputably, bad viewing conditions and longer intervals increase the difficulty of the identification task. Ambiguity though is a wider construct that incorporates additional elements which are known to influence decision making such as mood or the context (Loewenstein \& Small, 2007). 
either frequent or infrequent contact with black and white individuals, respectively. Supporting the contact hypothesis, participants with frequent contact with other-race individuals were less likely to exhibit ORB than participants with infrequent contact with other-race individuals. Similar findings have been reported for Chinese and Caucasian participants (Hancock \& Rhodes, 2008). Furthermore, training individuals to memorize or discriminate between own and other race faces is advantageous for other-race recognition (e.g., Hugenberg et al., 2010; Levin \& Beale, 2000). In line with the idea that interracial contact facilitates recognition of cross-racial characteristics and hence reduces ambiguity, we hypothesized that interracial contact would moderate blindness rates for other-race identifications.

To test our hypotheses we compared Black and White African and European participants' identification performance for simultaneous lineups. We hypothesized that other-race identifications would elicit higher blindness rates than own-race identifications due to the increased difficulty posed by the ORB to the latter group. However, Black participants were the only ones to make other-race identifications. Therefore, Black participants were expected to exhibit higher blindness rates than White participants. Additionally, blindness levels were expected to be moderated by the degree of interracial contact.

Aside from the effect of ORB on blindness rates, we tested two additional hypotheses about how blindness is related to target-present and target-absent lineups. Although earlier studies used target-present lineups exclusively (Chapters 3 \& 4; Sagana et al., 2013; Sagana et al., 2014b), in a recent study Sagana, Sauerland and Merckelbach (Chapter 5; 2015) presented participants with both target-absent and target-present lineups in order to provide a more complete picture of the effect. Specifically, they were interested in differences between participants who made a positive identification decision (i.e., choosers) and participants who rejected the lineup (i.e., nonchoosers). To that end, they manipulated both choosers' and nonchoosers' decisions. If participants had made a rejection, they turned this into a positive identification (i.e., choice reversal). If participants had made a positive identification, they swapped their choice with a different lineup member (i.e., choice exchange). They hypothesized that choice exchanges (i.e., nonchoosers, whose rejections were turned into identifications) would be more resistant to manipulations than choice reversals (i.e., choosers, whose identifications were interchanged). This assumption was based on the idea that when presented with the manipulated outcome, for choosers the comparison would rest on the specific facial characteristic of the identified suspect ("Did I identify this person or another person?"), while for nonchoosers the comparison would rest on a higher and less ambiguous decision level ("Did or did I not make an identification?"). Accordingly, the manipulation constitutes a within-category change for choosers but a between-category change for nonchoosers. Therefore, the mismatch between the 
action and outcome is greater for nonchoosers than for choosers, resulting in a detection advantage for nonchoosers compared with nonchoosers. The results supported their hypothesis; participants were seven to ten times less likely to detect choice exchanges (66.7\%) than choice reversals (11.2\%). The present study aims to replicate these findings in a laboratory setting. Therefore, we expected nonchoosers to exhibit lower blindness rates than choosers. Note that, following the Sagana et al.'s (Chapter 5; 2015) procedure, we refrained from turning identifications into rejections as such a conversion is of no practical relevance for legal proceedings. A rejection, even if it occurred as a result of blindness, would not qualify for a case to proceed to court.

Inaccurate identification decisions (foil selection or incorrect rejection) are interesting in this context. Inaccurate identifications imply a faint memory trace of the target, which adds another source of difficulty to the identification task. As such, we expected identification accuracy to moderate blindness for both choosers and nonchoosers. Earlier research lends some support to this assumption. Specifically, Sagana et al. (Chapter 4; 2013) reported that concurrent detectors were more accurate in their recognition decisions than blind participants. Note, however, that blindness phenomena cannot be reduced to weak or decayed memory (Chapters 3, 5 \& 9; Sagana et al., 2014a; Sagana et al., 2014b, 2015) as blindness is prominent even minutes after the original choice (Hall et al., 2012; Johansson et al., 2005) and with stimuli of personal and moral significance (Hall et al., 2013; Sauerland, Mehlkopf, Krix, \& Sagana, in press; Sauerland, Schell-Leugers, \& Sagana, 2015; Sauerland, Schell, et al., 2013).

\section{METHOD}

\section{Participants}

In total $N=123\left(M_{\text {age }}=24.4, S D_{\text {age }}=9.2\right.$, age range: $\left.18-65\right)$ participants took part in the study. Based on their ethnical background, participants were assigned to the following three groups: Black African ( $n=40,19$ men), White African ( $n=43,11$ men) and White European ( $n=40,9$ men). Participants were students $(82.4 \%$ ) of various majors (36.8.7\% arts and social sciences, $15.5 \%$ psychology, $3.1 \%$ mental health, $2.1 \%$ engineering) or worked in the public sector (5.9\%), private sector $(2.5 \%)$, and academia (4.2\%), or did not specify their background (5.0\%). Participants were tested at South-African and European university, respectively. The study was approved by the Ethical Committees of both universities. Participation was voluntary and participants received either no incentives or course credit in return for their participation. All participants were tested individually. 


\section{Materials}

\section{Stimulus films}

Four films showing minor offences with a mean duration of 3:07 minutes (duration range: 2:60 - 3:34 $\mathrm{min}$ ) were used as stimuli. Two films showed the theft of a wallet at a university cafeteria (Cafeteria1 \& Cafeteria2) and one film the theft of a wallet in a bar (Bar). The fourth film was about an assault attempt at a bus stop (Bus). In each film, there were four different actors: a perpetrator, a victim, and two bystanders. Given that we were mainly interested in the effect of ambiguity rather than ORB we consider is substantial to include only white targets in the films. Therefore, all targets were white Europeans from Germany and the Netherlands. Each target was in sight for at least $100 \mathrm{~s}$, with close-ups of 2 to $9 \mathrm{~s}$. All targets were shown from frontal and side views. The films were presented either in the order Cafeteria1, Bus, Bar, Cafeteria2 or Cafeteria2, Bar, Bus, Cafeteria1. Since detection rates $\left(\chi^{2} s \leq 3.09, p s \geq\right.$ .079 , phis $\leq 0.16)$ and identification accuracy $\left(\chi^{2} s \leq 7.95, p s \geq .070\right.$, phis $\left.\leq 0.25\right)$ did not differ as a function of video order, we will not discuss this factor any further.

\section{Lineups}

A total of 24 simultaneous photo lineups were created. For the four perpetrators and victims, both target-present and target-absent lineups were created. We were mainly interested in the four perpetrator identifications which are of the highest relevance in a criminal investigation and in lineup identification decisions. However, we also created target-absent and present lineups for the victim to avoid participants getting suspicious of the purpose of the study. For the bystanders, only target-present lineups were constructed and they were also used to mask the full purpose of the study. The uneven base rate of target-present and target-absent lineups might have increased the tendency of participants to choose. However, this difference could be controlled for due to the increased memory strength, given the small interval between watching the film and performing the identification. Thus, neither the victim nor the bystander lineups were analysed. To avoid anchoring effects, the target-present and targetabsent lineups were counterbalanced across videos and perpetrator and victim targets. Hence, for two videos the perpetrator lineups were target-absent and the victim lineups were target-present, whereas the reverse was true for the other two videos.

Head and shoulder photos were selected to match the description of the respective targets (effect sizes determined as Tredoux's Es ranged from 2.50 to 5.76; Tredoux, 1998; Tredoux, 1999). The pictures were presented on a $2 \times 3$ array and participants had unlimited time to make their choice. Thus, each lineup included one target (i.e., perpetrator, victim or bystander) or replacement of the target, and five distractors. On the right side of the screen, next to the five photos, participants were given the 
option to reject the lineup. The size of the photos, as presented on the computer screen, was $4 \mathrm{~cm} \times 5 \mathrm{~cm}$.

\section{Questionnaires}

Interracial Contact Questionnaire. The interracial contact questionnaire (Sigelman \& Welch, 1993) investigates the degree of contact black individuals have with white individuals and vice versa. The questionnaire consists of five questions. The first is a yes/no question about whether participants have a close white (black) friend. Then, a pair of questions about the racial composition of their current neighbourhood and elementary or secondary school follows. Participants use a response scale of "all white/black", "mostly white/black", "about half black and half white", "mostly black/ white", and "all black/white", which we coded on a 0-4 scale. This coding scheme deviates from that of Sigelman and Welch (1993) who assigned the "all white/black" and the "mostly white/black" to a single category resulting in a three-point scale. However, this is a self-report questionnaire and hence the distance between the two answers may reflect large quantitative and qualitative differences. We think the present coding scheme is more sensitive to these variations. The final two questions are about the level of interaction between adults and children of different races. These response options are "a great deal" (= 3), "only somewhat" (= 2), "hardly ever" (=1), "no members of the other race in the neighbourhood" (=0). The first question (interracial friendship) is used as a separate measure of contact. A total score (range: 0-14) was calculated by summing up the four racial composition and interaction questions.

Post-Test Questionnaire. Participants were given a post-test questionnaire to explore whether they had noticed the manipulation but refrained from revealing it. Participants were first asked whether they had noticed anything strange during the experiment. If they responded affirmatively, they were invited to provide details. Next, participants were misleadingly informed that the present study employed two experimental conditions, one in which some of the choices were manipulated (as in the actual experiment), and one control condition, where this was not the case. Participants had to indicate to which condition they believed they had been assigned. If a participant indicated that $s /$ he belonged to the manipulated condition, $s /$ he also had to indicate how many times $\mathrm{s} /$ he had noticed a manipulation (Chapter 4; Sagana et al., 2013; Sauerland, Schell, et al., 2013).

\section{Design}

For this study we employed a mixed 3 (race: Black African vs. White African vs. White European; between subjects) $\times 2$ (manipulation: manipulated vs. non-manipulated lineup; within subjects) x 2 (lineup mode: target-present vs. target-absent; within subjects) factorial design. Dependent variables were detection rate (concurrent and 
retrospective) and identification accuracy. Concurrent detection was coded, when a participant immediately noticed the manipulation. This includes participants who indicated that they noticed the change in their statements about the reasons for their decision or report to the experimenter. Retrospective detection was coded when a manipulation was detected concurrently and when a participant reported the manipulation in the post-test questionnaire.

\section{Procedure}

All video fragments, instructions, and lineups were programmed with DMDX Display Software (Forster \& Forster, 2003) and were presented on a 15 inch computer screen at a resolution of $1024 \times 768$ pixels. Participants were naïve to the actual purpose of the study and were tested individually.

The experiment involved two sessions. In session 1, participants watched the first video fragment and immediately after its end they were asked to identify all four actors from separate photo lineups, always in the order perpetrator, victim, and two bystanders. Participants had unlimited time to make their decision. Participants were told that the target may or may not be present and were given the option to reject the lineup. The same procedure was followed for the remaining three video fragments. After participants had finished with the identifications for all four films, session 1 was terminated.

In Session 2, $48 \mathrm{hrs}$ later, participants were instructed to imagine that they were in a court room, with the victim and a suspect present. The photo of the victim was presented in order to provide a context and to avoid confusion between the four video fragments. Participants were further instructed that the judge would ask them to explain why they had made a selection or rejection from the perpetrator lineup in session 1. Then, participants were presented with the face of the identified suspect and had to write down their reasons on paper sheets provided in front of them. If participants had rejected the lineup, they were presented with a sketch face and were asked to explain why they had rejected the lineup. The same procedure was followed for all four video fragments. However, for two of the four suspects, participants' identification decisions were manipulated. That is, participants were presented with a lineup member different from the one they had chosen two days earlier. Which lineup member was presented was predetermined by the experimenter. If participants had initially rejected the lineup, they were presented with the photo of the perpetrator in the target-present condition and the photo of the replacement in the target-absent condition, instead of the sketch photo. Once participants finished this task for all four videos, they were asked to fill in the post-test questionnaire. Finally, participants were thanked and fully debriefed. 


\section{RESULTS}

An alpha level of .05 was used for all analyses. Recall that our analyses focus on the four perpetrator identifications alone.

\section{Own-Race Bias}

As a first step we examined whether ORB was evident in our study. We expected identification accuracy to be better for own- compared with the other-race identifications. To examine whether identification accuracy was dependent on race we applied a regression methodology known as Generalized Estimating Equations (GEE) analysis. The method enables the incorporation of the repeated observations for all the perpetrator lineups leading to a total $N$ of $4 \times 123=492$ cases. Contrary to our expectations, identification accuracy did not differ as a function of race, Wald $\chi^{2}(2, N=492)=2.75$, $p=.253$. Therefore, no ORB was evident in our study.

\section{Blindness for One's Own Identification Decision}

In total, 2 × $40=80$ manipulations were performed with Black Africans, 2 × $43=86$ manipulations with White Africans, and $2 \times 40=80$ manipulations with White European participants. Separate GEE analyses were performed for concurrent and retrospective detection as outcome variables, and with racial group and interracial contact (i.e., interracial friendship and total score) as predictors.

For concurrent detection, the univariate distribution ( $N$ of $2 \times 123=246$ cases) was markedly skewed with only 32 instances (13\%) of detection. This low number of detections puts restrictions on the complexity of GEE models that can be studied (following the guidelines of Peduzzi, Concato, Kemper, Holford, \& Feinstein, 1996) in order to avoid overfitting. Furthermore, the inclusion of interracial friendship and race by interracial friendship interaction led to high collinearity and associated problems in parameter estimation. Thus, we examined a model containing only race, interracial contact, and the race by interracial contact interaction as possible predictors. The interaction effect between race and interracial contact was non-significant, Wald $\chi^{2}(2$, $N=246)=2.03, p=.362$. After deletion of this interaction term, the results showed a non-significant main effect for interracial contact, Wald $\chi^{2}(1, N=246)=1.05, p=.306$, but revealed a significant race effect. After deleting the non-significant interracial contact effect, race attained significance with Wald $\chi^{2}(2, N=246)=7.23, p=.027$. Closer investigation of the race effect showed a significant difference between Black Africans and White Europeans, Wald $\chi^{2}(1, N=246)=7.21, p=.007$, but no significant difference between White Africans and White Europeans, Wald $\chi^{2}(1, N=246)=1.46$, $p=.226$. Inspection of the associated odds ratio showed White Europeans to be approximately five times more likely to detect a manipulation than Black Africans. Thus, 
as expected other-race identifications elicited higher blindness rates than own-race identifications. Unexpectedly, however, blindness levels were not moderated by the degree of interracial contact.

For retrospective detection, the univariate distribution $(N=246)$ was more symmetrical with $39 \%$ of detections. That permitted the investigation of a more complicated GEE model containing race, interracial contact, and interracial friendship as main effects, and two-way interactions with race as terms. However, the model yielded non-significant effects, Wald $\chi^{2} s(1-2, N s=246) \leq 1.43, p s \geq .488$. Sequential removal of non-significant interaction terms led to non-significant main effects, Wald $\chi^{2} s(1-2$, $N s=246) \leq 2.16, p s \geq .339$. The sequential removal of the least significant effects did not alter the pattern of findings. Although race did not have a significant effect on retrospective detection, the sample odds ratios were in line with our expectations. White Europeans were 1.5 times more likely to retrospectively detect a manipulation than Black Africans, whereas this likelihood of detection was similar for White European and White African participants (odds ratio .85). Figure 6.1 displays concurrent and retrospective detection rates across racial groups.

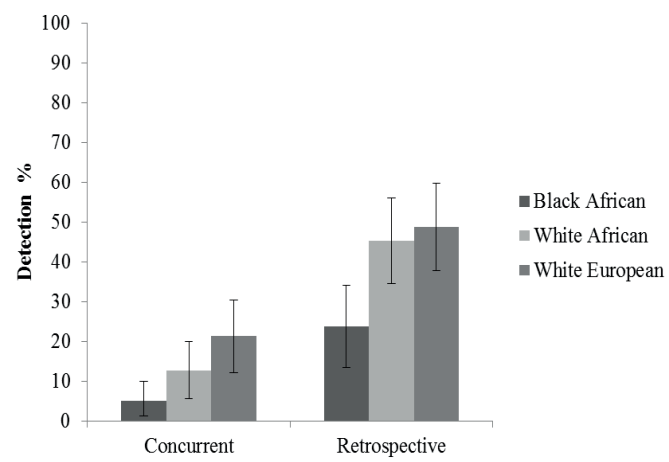

Figure 6.1 | Mean detection rates for the four perpetrator lineups across racial groups. Error bars represent $95 \%$ confidence intervals.

\section{Blindness as a Function of Identification Accuracy and Choosing}

Next, we tested the hypothesis that detection rates would be elevated for nonchoosers compared with choosers and that this difference depends on identification accuracy. For this purpose we conducted separate GEE analyses for concurrent and retrospective detection as dependent variables and choice (choosers vs. nonchoosers) and accuracy (correct vs. incorrect) as predictors. For both types of detection, the pattern of results was similar. The interaction effects between choice and accuracy were nonsignificant, Wald $\chi^{2} s(1, N s=246) \leq 1.55, p s \geq .214$. A model including only the main 
effects also revealed no significant effects, Wald $\chi^{2} s(1, N s=246) \leq 0.85, p s \geq .356$. By and large our hypotheses regarding choosing and accuracy were not supported.

\section{DISCUSSION}

The aim of the present study was to investigate the impact of ORB on blindness for one's own eyewitness identification decisions. We hypothesized that making otherrace identifications would increase blindness rates as compared with own-race identifications. We based this prediction on the fact that other-race identifications are more difficult as they require more cognitive effort (Brigham \& Malpass, 1985; Chiroro \& Valentine, 1995). The results support our prediction. Participants who performed other-race identifications, concurrently displayed higher blindness rates, compared with those who made own-race identifications. Retrospectively, blindness decreased for own and other-race identifications, yet remained alarmingly high (see Figure 6.1). These rates resembled those found in earlier studies on blindness for facial recognition decisions (concurrent: $30-31 \%$, retrospective: 59-61\%, Chapters 3 \& 4; Sagana et al., 2013; Sagana et al., 2014b). Note, however, that differences between groups in retrospective detection rates followed a pattern that was in line with our hypothesis, but remained non-significant.

Peculiarly, we found a difference in detection rates across racial groups in the absence of a (statistical) ORB effect. Although the lack of ORB is not in line with our expectations, it might relate to the fact that other-race identifications referred only to Black participants identifying white targets. This was done because we were primarily interested in ambiguity and to keep the complexity of the experiment manageable. We anticipated though that blindness would be even greater when white eyewitnesses have to identify black targets. Past research revealed that white participants are more likely to demonstrate the ORB effect, compared with other-race participants (e.g., Anthony, Copper, \& Mullen, 1992; Horry, Wright, \& Tredoux, 2010; Hourihan, Benjamin, \& Liu, 2012). Importantly, the increased blindness for other- versus ownrace identifications speaks to a difficulty in discriminating between previously seen and novel faces is greater for other- compared with own-race faces (Arnold, 2013). Participants who perform own-race identifications may have increased capacity for monitoring whether the originally identified and the manipulated lineup member were or were not the same person, compared with participants who perform otherrace identification. Therefore, participants who perform own-race identifications may find it easier to detect the manipulation.

An alternative explanation for our findings may be that Black participants have a higher tendency to answer in social desirable ways. Although we cannot rule 
entirely the explanation of cultural differences responsible for the higher blindness rates among black participants we consider it unlikely. Early research indicates higher social desirability for black compared with white participants (Fisher, 1967; Klassen, Hornstra, \& Anderson, 1975). However, newer studies have challenged these findings (for a review see Johnson \& Van de Vijver, 2003; Welte \& Russell, 1993). Specifically, black individuals use social desirable answers to overcome differences in social power. Nowadays however, the differences between white and black individuals are attenuated. Thus, we doubt that this is a valid explanation for our findings. Furthermore, it is important to note that compliance, suggestibility, and other personality characteristics do not sufficiently account for blindness phenomena (Merckelbach et al., 2011a; Chapter 2; Sauerland, Sagana, et al., 2013; Sauerland, Schell, et al., 2013).

Interestingly, interracial contact was not diagnostic of concurrent detection for any racial group. A possible explanation for this finding could be the concentration of total interracial contact scores around the median and hence a lack of variability. For retrospective detection though interracial friendship (defined as yes or no) generated similar results. Alternatively, we cannot rule out the possibility that existing differences were masked by the use of a questionnaire that accounts for the quantity rather than the quality of interracial contact. Evidently, social cognitive factors such as motivation to differentiate other-race individuals (Levin, 2000; Pauker, Rule, \& Ambady, 2010) may be a stronger moderator of ORB. Taken together, our findings suggest that increased interracial contact is not sufficient to overcome blindness for one's identification decisions for other-race identifications. Thus, it seems that blindness phenomena have a strong cognitive basis that social factors cannot mitigate easily.

Our final hypothesis was derived from the assumption that turning lineup rejections into identifications constitutes a more profound change than an alteration in the identity of a chosen target (i.e., turning a rejection into a choice vs. switching the selected lineup member). Accordingly, we anticipated that nonchoosers' detection rates would be higher than choosers' detection rates. However, and in contrast to previous findings (Chapter 5; Sagana et al., 2015) the findings did not support our hypothesis. Making a positive identification or rejecting the lineup was not predictive of concurrent and retrospective detection. This counterintuitive finding awaits replication because it is of profound relevance to the legal setting. We will return to this point later in the discussion. Nonetheless, our results may derive from the fact that we did not discriminate between "not present" and "don't know" answers. It is plausible that participants who did not have a clear memory of the target (don't know) opted for the rejection option (Sauerland et al., 2012; Sauerland \& Sporer, 2009). These doubtful participants may be more likely to accept the manipulated outcome. Even if we assume that all inaccurate nonchoosers would select the "don't 
know" option, this is not sufficient to support the claim that detection rates would be increased as identification accuracy was not diagnostic of detection.

Relatedly, we failed to find any basis for our assumption that identification accuracy moderates blindness. However, our failure to obtain such effect is in line with previous studies indicating that blindness for one's identification decisions cannot simply be attributed to poor memory for the target or forgetting (Chapters 3, 5 \& 9; Sagana et al., 2014a, 2014b; Sagana et al., 2015). This findings is also consistent with recent results indicating that blind participants are as able as detectors to recall their original choices when they are asked to remember (Chapter 9; Sagana et al., 2014a); a finding that speaks against the idea that participants who accept the manipulation are those with the weakest memory. Likewise, blindness for eyewitness recognition decisions has not (Chapters 3 \& 5; Sagana et al., 2015) or only partly been shown to be driven by memory strength (Chapter 4; Sagana et al., 2013).

From an applied point of view our results imply that a suspect could theoretically be prosecuted based on a manipulated identification that was accepted, even though the eyewitness originally rejected the lineup. The importance and possible devastating consequences of this finding are indisputable. It is not hard to imagine a situation where a suspect targeted by the police, yet innocent, is placed in a lineup. Investigating a case requires high involvement that at times may result in tunnel vision (Findley \& Scott, 2006; Leo \& Drizin, 2010). Tunnel vision may lead the investigator to focus only on facts that are in support of the original hypothesis (i.e., the guilt of the suspect) and dismiss the non-supportive ones. Consider this hypothetical scenario: From the investigator's perspective, a lineup rejection in a case reflects the eyewitness missing an "obvious" cue. The investigator thinks that this omission undermines the progress of the investigation. Hence, following a lineup rejection, the investigator decides to turn the rejection into a suspect identification. Considering the present and previous findings, one must worry that a substantial proportion of eyewitnesses would disregard the change in the nature of their decision. Having said that, it is worth mentioning that although few - to date - there are real cases where similar changes have been reported (see Chapter 3; Sagana et al., 2014b). Importantly and in addition to the aforementioned implications, the mere fact that an eyewitness performs otherrace identification increases the risk of eliciting blindness. The fact that $40 \%$ of the wrongful conviction cases that have been attributed to eyewitness misidentifications were cross-racial identifications (DNA Exonerations Nationwide, 2013), emphasizes the relevance of the present findings.

The current findings also add to our theoretical knowledge about blindness phenomena. Specifically, we reasoned that increased situational difficult induces ambiguity which in turn fosters blindness (Merckelbach et al., 2011a). Although we did not directly test difficulty, we considered other-race identifications as more challenging 
than own-race identifications due to differences in encoding. The increased blindness rates for other-compared with own-race identifications support the difficulty hypothesis and favour ambiguity as moderator of choice blindness. On the other hand, the absence of differences in blindness for choosers and nonchoosers in the own-race identifications participants weakens an ambiguity interpretation. That is because for nonchoosers the comparison rests on a higher and less ambiguous decision level ("Did or did I not make an identification?") as compared with choosers for whom the comparison rests on the specific facial characteristic of the identified suspect ("Did I identify this person or another person?"). Thus, our findings are somewhat inconsistent as to the role of ambiguity as a moderator of blindness. Therefore, future research should focus on cognitive and neurobiological explanation for the occurrence of the effect.

The limitations of the current study deserve some comment. First, as was pointed above, the design was asymmetrical. Thus, white participants were not asked to identify black suspects. This asymmetry may complicate an interpretation of our results as group differences may not only reflect the difficulties of other-race identifications but also differences in effort. Thus, future studies should preferably look at blindness for identification decisions with more symmetrical designs and across other races. A second limitation is that we did not discriminate between "not present" and "don't know" answers. For reasons already explained we would not expect replication difficulties by addition of the "don't know" option. Nevertheless, this is for future research to explore.

To sum up, the present study indicates that blindness for identification decisions is prominent and can occur for own- and other-race identifications. Additionally, it demonstrates yet again that blindness occurs not only for lineup identifications, but also for rejections. These findings are highly relevant for practice as they indicate a previously unstudied pathway to the conviction of innocents. Until now, we are not in a position to estimate the exact magnitude of the effect in real cases. However, there are real life cases illustrating that blindness for identification decisions is not at all that improbable (see Chapter 3; Sagana et al., 2014b for details). Certainly, future research is to determine the magnitude of the effect in real cases. This could be a long and hard process as a "leaving no traces" principle (i.e., people are not aware of the alternated outcome as they are blind to the change) is an integral component of blindness. Till then though, ignoring the possibility that choice blindness pose threat to identification decisions, may not be wise. 



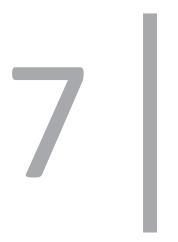

Witnesses' failure to detect covert manipulations in their written statements 


\section{ABSTRACT}

Considering that law enforcement agencies and legal professionals often have to rely on witness statements, we examined whether witnesses can detect errors in their written statements. In three experiments, participants watched a mock-crime film and provided a written statement of what they had witnessed. Following a delay of several minutes (Experiment 1), $48 \mathrm{hr}$ (Experiment 2), or 1 month (Experiment 3), participants were exposed to and interviewed about their testimony. Unknown to them, they were confronted with statements which included four secretly manipulated details. Participants' ability to detect these manipulations varied as a function of delay (Experiments 1/2: 74-89\%; Experiment 3: 36-52\%). For the shorter delays, manipulations of plot details were more likely to be detected than manipulations of incidental details. The poor ability of witnesses to detect manipulations in their written statements could impede the accurate reconstruction of criminal acts and lead to incrimination of an innocent person. 


\section{INTRODUCTION}

In 1988, Herman Atkins was convicted of rape based on a witness statement connecting him to the area of the crime. Atkins' over 11 years-long imprisonment was terminated in 2000 after DNA evidence proved his innocence. However, it was yet to be revealed that a detective involved in the case had fabricated the witness statement that led to his conviction. The Atkins case can be interpreted as an example of prosecutorial misconduct and reflects only a fraction of the documented misconduct incidents (see Ridolfi \& Possley, 2010). Earlier research has shown that police sometimes resort to techniques such as changing or omitting details from witnesses' statements to elicit a confession (Pearse \& Gudjonsson, 1999). Taken together, these findings raise the fundamental question whether witnesses can detect manipulations in written reports of their statements.

Eyewitnesses are notorious for their poor ability to filter out misleading post-event information (e.g., Gabbert, Memon, Allan, \& Wright, 2004; Loftus, 2005; Loftus \& Hoffman, 1989). In a typical misinformation paradigm, after watching a staged crime, participants read a narrative description of the event that contains misleading information. Then, participants are provided either with a recall or a recognition task to determine whether the misinformation has affected the original memory of the event. However, the misleading information is often not tailored to the memory of each participant. Therefore, participants may fail to detect the misinformation because it refers to details that were never encoded in the first place (Lindsay, 1993; McCloskey \& Zaragoza, 1985b).

However, detecting misinformation in others' written accounts is substantially different from detecting changes in one's own statement. Providing a statement requires witnesses to deliberate and evaluate the strength of their memory in an attempt to decide which details they should report (Goldsmith et al., 2002). Therefore, all the reported details are clearly part of their memory. Thus, it could be argued that in the case of their own testimony, witnesses should be able to detect manipulations in their statements.

Germane to this issue are studies on the accuracy of autobiographical memories written down as diary entries (Barclay \& Wellman, 1986; Merckelbach et al., 1997). These studies had participants write down diary entries about memorable, yet ordinary activities, after which they performed a recognition test consisting of original and manipulated diary items. Participants were found to be good at recognizing original autobiographical fragments, but they displayed a tendency to accept the manipulated fragments as genuine memories. Furthermore, as the time interval between the diary entries and the recognition tests became longer, this tendency became more pronounced. However, these results were obtained in the context of diaries. Hence, the 
acceptance of manipulated fragments might reflect the liberal recognition thresholds that people rely on in this setting. Obviously, the eyewitness context is different from that of autobiographical notes.

Relevant for the eyewitness context is the work of Christianson, Engelberg, and Gustafson (Experiment 1; 2007) who examined how different methods of presenting transcribed versions of witnesses' statements affect their tendency to detect errors in their testimony. After watching a mock crime film, participants were questioned and their statements were subsequently transcribed. While typing the statements, the experimenter inserted six manipulations into the original report. Participants were then asked to inspect the statements for mistakes either by listening to the experimenter reading the statement or by reading the statement out-loud themselves. Participants detected more errors when they read the statement themselves (detection rate: 4.33) than when they listened to the statement being read to them (detection rate: 0.93 ). Although their aim was not to examine eyewitnesses' sensitivity to manipulations in their statement reports, Christianson et al.'s (Experiment 1 ; 2007) findings suggest that witnesses sometimes accept unreported details as their own testimony, even when they are requested to search actively for errors.

The failure of witnesses to detect manipulations in their statements may have serious consequences for legal decision making. Undetected manipulations in official legal witness documents could compromise the accurate reconstruction of a criminal act. Such undetected errors could cause ambiguity about the sequence of events and the key features of the perpetrator, and possibly lead to an innocent suspect being incriminated. Furthermore, due to an inaccurate reconstruction, important physical evidence may be lost, and police investigation could be delayed. That unintended manipulations of witness statements occur on a regular basis is highly likely. For example, Malsch, Haket, and Nijboer (2008) had police officers produce official statements of a videotaped interrogation of 2451 words. The resulting statements varied between 980 and 1464 words, which implies that the officers summarized the original. Such summaries open the door for misinterpretations.

Given the devastating potential of undetected errors in witnesses' reports, we examined witnesses' ability to detect manipulations in their written statements. In real cases, eyewitnesses are given the opportunity to check whether the transcribed version of their statement contains any mistakes after giving testimony. They are also confronted with their statements in court, months or even years after providing the statement. Older memories, however, are harder to retrieve than more recent memories (Crovitz \& Schiffman, 2013; Read \& Connolly, 2007; Wixted \& Ebbesen, 1991). Furthermore, extensive research indicates that the longer the time interval between misinformation and the target event, the more effective the misinformation is (Belli, Windschitl, McCarthy, \& Winfrey, 1992; Frost, Ingraham, \& Wilson, 2002; Higham, 
1998; Loftus, 2005; Underwood \& Pezdek, 1998). For example, Belli et al. (1992) noted that participants who were exposed to misleading information one week after witnessing a target event were more susceptible to it than those who had been exposed to misinformation a few minutes after the target event. The authors concluded that after the longer delay, a substantial amount of forgetting has occurred, leading to greater susceptibility to post-event suggestions (see also Brainerd, Reyna, Howe, Kingma, \& Guttentag, 1990). Similarly, Underwood and Pezdek (1998) observed that participants falsely recognized more misleading suggestions when a recognition test was presented one month rather than $10 \mathrm{~min}$ after exposure to misinformation, if they felt that the source of misinformation was reliable. Therefore, considering different time intervals in participants' ability to detect manipulations in their written statements is theoretically relevant and has important practical implications. With this consideration in mind, we performed three experiments in which participants first provided a written statement and were later interviewed about it. During the interview, participants were confronted with statements that included up to four covertly manipulated details. The procedure was similar across our experiments except that the confrontation with the manipulated statement occurred several minutes (Experiment 1), $48 \mathrm{hr}$ (Experiment 2), or one month (Experiment 3) after giving testimony. Considering that longer retention intervals are associated with greater memory decline (Crovitz \& Schiffman, 2013; Read \& Connolly, 2007; Wixted \& Ebbesen, 1991) and increased susceptibility to misinformation (Belli et al., 1992; Higham, 1998; Loftus, 2005; Underwood \& Pezdek, 1998), participants' ability to remember the content of their statements with precision should decrease over time. Thus, we expected that participants would be less able to detect manipulations in their statements for longer than for shorter intervals between the testimony and the presentation of the manipulated statement.

We also explored the possibility that failure to detect statement manipulations is dependent on the significance of the manipulated detail. According to the attentional narrowing hypothesis, during the processing of emotional events, attention is largely focused on the essential rather than the less relevant details (Christianson, 1992; Christianson \& Loftus, 1987, 1991; Wessel \& Merckelbach, 1998). As a consequence, memory for essential information is stronger compared with memory for incidental information. Hence, participants should be more likely to detect manipulations of well-preserved rather than poorly preserved information in memory. Therefore, we expected that detection rates would be higher for manipulations of essential instead of incidental details. Furthermore, the predicted detection advantage of essential over incidental details should prevail across retention intervals, as both types of details would be subject to memory decay over time. One problematic aspect in testing predictions that flow from the attentional narrowing hypothesis is that it remains dif- 
ficult to define a priori essential and incidental details. For the purpose of this study, we classified details based on their relevance to the course of the witnessed event (Dalton \& Daneman, 2006; Wright \& Stroud, 1998). That is, details that were essential to the course of the witnessed event were defined as plot details, while subsidiary to the course of events details were defined as incidental. As an additional indicator of relevance to the course of events we used the frequency that those details were reported during free recall (e.g., Heath \& Erickson, 1998; Saunders, 2009).

To sum up, the present work tested to what extent witnesses are capable of detecting manipulations in their written statements at a variety of retention intervals. First, we expected considerably low detection rates across experiments. Second, we anticipated decreased detection rates as retention intervals increase. Additionally, we hypothesized that the ability to detect a manipulation would be more pronounced for plot than incidental details across experiments and delay conditions.

\section{METHOD}

The testing materials and procedure were largely analogous across experiments, with the exception of the retention interval between providing the statement and being presented with the manipulated version. Retention intervals were several minutes (i.e., < $30 \mathrm{~min}$, Experiment 1), $48 \mathrm{hr}$ (Experiment 2), or one month (Experiment 3). All reported studies were approved by the standing ethical board of the faculty.

\section{Participants}

In Experiments 1 and 2, participants were 29 and 40 (22 men, $M_{\text {age }}=21.2$ years, $S D_{\text {age }}$ $=3.2$, age range: $18-41)$ students of German $(n=35)$ and Dutch $(n=34)$ origin. Most participants were Bachelor students at the faculty of Psychology and Neuroscience (92.8\%) or other faculties (7.2\%). In Experiment 3, participants were 32 Germans (6 men, $M_{\text {age }}=21.1$ years, $S D_{\text {age }}=2.9$, age range: $\left.18-30\right)$. All were Bachelor students at the Faculty of Psychology and Neuroscience (93.5\%), except for two individuals (who were working in the private sector). Participation was voluntary in exchange for course credit or a gift certificate. All participants were naïve to the purpose of the study and were tested individually in their native language.

\section{Materials}

Stimulus film

A film fragment depicting a staged assault attempt was displayed on a computer screen with a resolution of $768 \times 1024$ pixels. The duration of the fragment was $2 \mathrm{~min}$ 
$43 \mathrm{~s}$ and comprised the following plot: A young red-haired woman with a fuller figure (i.e., the victim) walks down the road with two friends (bystanders) who are pushing their bicycles. The bystanders are a slim, tall woman with brown hair and a young, tall man with brown hair. All three arrive at a bus station where a young man with a fuller figure and black hair (i.e., the perpetrator) is sitting on a bench. After the victim tells her friends that the bus she has to catch should arrive within minutes, the bystanders ride away. On their departure, the perpetrator approaches the victim and invites her out for a drink. Her negative response turns the perpetrator violent. He grabs and then pushes the victim into the bus stop. The victim manages to escape and runs away entering a park behind the bus stop. However, the perpetrator catches up with her and here the fragment ends.

\section{Manipulations}

We performed four manipulations, two concerning plot and two concerning incidental details. Our a priori classification of the details was based on their relevance to the course of the witnessed event (Dalton \& Daneman, 2006; Wright \& Stroud, 1998). Specifically, details that are essential to the course of the witnessed event were classified as plot details and those that are subsidiary to the course of the event were classified as incidental details. Additional evidence for our classification comes from a pilot study in which we looked at the frequency with which event details were provided in participants' free recall $\left(N=21,4\right.$ men, $M_{\text {age }}=27.0, S D_{\text {age }}=11.7$, age range: $21-53)$. Thus we manipulated the following features: a) the perpetrator pushed the victim to the ground ( $M=71 \%, 95 \% \mathrm{Cl}[50.0,86.2]$; plot), b) the surroundings of the scene was a parking lot $(M=66 \%, 95 \% \mathrm{Cl}[45.4,82.8]$; plot), c) the hair colour of the female bystander was red $(M=43 \%, 95 \% \mathrm{Cl}[24.2,63.5]$; incidental), and d) the figure of the victim was normal ( $M=33 \%, 95 \% \mathrm{Cl}[17.2,54.6]$; incidental). However, because in Experiments 1 and 2, the detection rate between the two plot details differed significantly ( $p s \leq .003$ ), in Experiment 3 we changed one of the two plot detail manipulations. Specifically, instead of changing the surrounding of the scene into a parking lot, we changed the action of the two bystanders from riding away on their bikes into walking away ( $M=80 \%, 95 \% \mathrm{Cl}[60.0,92.3]$; plot).

Paired comparisons performed on the pilot data revealed significant differences in report frequency between the plot and the incidental details, McNemar $\chi^{2} s(1, N=$ $21) \geq 4.17$, $p s \leq .031$, with exception the comparison between the first plot and the third incidental detail $(p=.062)$. No differences occurred within each detail category, McNemar $\chi^{2} s(1, N=21) \leq 0.50, p s \geq .480$. Because not all participants reported the pre-selected details, the a priori categorization of the details to-be-manipulated resulted in loss of data points. However, refraining from a priori categorization would have introduced variation in the manipulated details, which would have made 
interpretation of the results difficult. Furthermore, we did not add information to participants' statements. If a critical to-be-manipulated detail was not reported, we did not perform a manipulation for that detail.

\section{Post-test questionnaire}

The post-test questionnaire aimed to assess whether participants had noticed the manipulations in their statements, but were reluctant to reveal them during the interview. First, participants were asked three open-ended questions as to whether they had noticed any problems or anything strange during the experiment. Then, they were misinformed that the study employed two conditions: an experimental condition where some details in participants' statements had been manipulated, and a control condition where no manipulations had been made. Participants had to indicate in which condition they thought they had been assigned to. If they thought they had been in the experimental condition, they had to specify which details they believed had been manipulated. If these responses were correct, they were counted as retrospective detection.

\section{Design}

We employed a within-subjects design with detail relevance (plot vs. incidental) as the independent variable. Concurrent and retrospective detection served as the dependent variables. Concurrent detection occurs when concerns about the integrity of statement details are raised at the time of presentation (i.e., during the interview). Retrospective detection additionally includes instances of suspicion that are expressed after the interview in the post-test questionnaire. Thus, all concurrent detections are automatically also counted as retrospective detections. Finally, the memorability of the details was assessed by coding the accuracy (accurate vs. inaccurate) of the later to-be-manipulated details in participants' reports.

\section{Procedure}

The experiment consisted of two parts. In part 1, participants provided a witness statement. In part 2, they were interviewed about 10 details in their statements, four of which (at a maximum) had been manipulated. The purpose of the interview was to examine whether participants would raise concerns about the accuracy of the statement when their attention was pointed to specific details. A cover story indicating that the study investigated the link between personality and memory was used to mask the real purpose of the study.

During the first part, after signing the informed consent form, participants watched the stimulus film and then filled out personality questionnaires as a filler task. Next to allowing for a time interval between the film and preparing the statement, the 
filler task served to strengthen the credibility of our cover story. No time limit was imposed for the filler task. Hereafter, participants of all three Experiments were given unlimited time to provide a free report about the witnessed crime. They were instructed to follow the chronological sequence of the events and to describe all people involved in the film. Furthermore, they were discouraged from guessing about details they could not remember (Gabbert, Hope, \& Fisher, 2009; Hastie, Landsman, \& Loftus, 1978). Participants typed their statement in a text file, saved it in a designated computer folder and then printed the statement. This procedure was followed to give participants the impression that they had control over their statement. Finally, participants either continued with a working memory computer task (Experiment 1 ) or were thanked and dismissed (Experiment 2/3).

In Experiment 1, while participants were busy with the computer task, the experimenter covertly accessed the digital file with their statements from another computer and manipulated the critical details as described above. Then s/he printed and saved the manipulated version of the statement in the same location and with the same name as the original one. In Experiments 2 and 3, the same procedure was followed after participants had left the lab.

Part 2 took place on completion of the computer task $(<30 \mathrm{~min}$ ) for participants in Experiment 1, while participants returned to the lab $48 \mathrm{hrs}$ later in Experiment 2 and one month $\left(M_{\text {days }}=28.7, S D_{\text {days }}=1.6\right)$ later in Experiment 3. In Experiment 3, we allowed a deviation of two days to accommodate participants' classes and weekends.

The interview started with the experimenter instructing participants to open their statement on the computer screen and handing them the manipulated printed version. Participants were first asked to read their statement as a reminder. Subsequently, the experimenter inquired about the four manipulated (e.g., "In your statement you mention that the victim was of normal weight. Can you estimate her weight in kg?") and six non-manipulated details. If participants had not mentioned the detail in their original statement, no manipulation was performed. However, to keep the length and the content of the interview constant across participants, the interviewer formulated a question related to the non-reported detail in general terms (e.g., "Could you estimate the height and the weight of the victim?"). If participants, at any point of the interview, raised concerns about the manipulated details (e.g., "Well, she was a bit overweight"), the experimenter first referred them to their statement. If they insisted, then the experimenter kept a neutral position (e.g., "If you feel there is a mistake, I will make a note and check it later. Shall we continue with the interview?") and continued with the interview. All instances of suspicion (major and minor), if correct, were counted as concurrent detection. After the interview, participants were asked to fill out the post-test questionnaire, were thanked and debriefed. 


\section{RESULTS}

\section{Detection Rates}

As many participants did not report all to-be-manipulated details in their original statements, we could change fewer details than initially planned. In Experiment 1 , of the $29 \times 4=116$ planned manipulations, we could only change 82 details ( 49 plot and 33 incidental details). Of these, 61 (74.4\%) were detected concurrently and 73 (89.0\%) retrospectively. In Experiment 2, we performed 108 (66 plot and 42 incidental details) of 160 planned manipulations. Eighty-one (75.0\%) were detected concurrently and 95 (88.0\%) retrospectively. In Experiment 3, we performed 78 (45 plot and 33 incidental details) of 128 planned manipulations. Twenty-nine (37.2\%) were detected concurrently and 42 (53.8\%) retrospectively.

To examine whether the retention interval between the statement and presenting the manipulated outcome affected detection rates, we meta-analysed the mean detection rate of the three experiments $(k=3)$. Our analysis revealed no differences in detection rates across Experiments 1 and 2 for concurrent (fixed effects $d=0.74$ ), $Q(1)=0.02, p=.876, l^{2}=0.0 \%$, and retrospective detection (fixed effects $d=0.88$ ), $Q(1)=0.04, p=.831, I^{2}=0.0 \%$. Adding Experiment 3 to the analysis significantly increased the heterogeneity for concurrent (fixed effects $d=0.65$ ), $Q(2)=35.04, p$ $<.001, l^{2}=94.3 \%$, and retrospective detection (fixed effects $d=0.83$ ), $Q(2)=31.48$, $p<.001, l^{2}=93.65 \%{ }^{4}$. Evidently, the long retention interval between providing the statement and the confrontation with the manipulations resulted in a substantial decrease in detection rates. Given that detection rates in Experiments 1 and 2 were highly comparable, we collapsed the data for all subsequent comparisons. Running all analyses separately for Experiment 1 and 2 led to a similar pattern of results, with no exception. We therefore describe the analyses based on the pooled sample.

\section{Accuracy of Witness Memory}

In Experiments 1 and 2, the recall accuracy of the later to-be-manipulated details was high (97.3\%). Of the five errors that occurred in total, one referred to plot and four to incidental details. Manipulations of all five inaccurately recalled details were detected concurrently. In Experiment 3, recall accuracy of the later to-be-manipulated details

$4 \quad$ Running the analysis only for the three manipulated details that remained consistent across Experiments did not change the pattern of findings. Specifically, there were no differences in detection rates across Experiments 1 and 2 for concurrent (fixed effects $d=0.72$ ), $Q(1)=0.62, p=.432, I^{2}=$ $0.0 \%$, and retrospective detection (fixed effects $d=0.87$ ), $Q(1)=0.85, p=.354, l^{2}=0.0 \%$. Adding Experiment 3 to the analysis significantly increased the heterogeneity for concurrent (fixed effects $d$ $=0.66), Q(2)=12.19, p=.002, I^{2}=83.6 \%$, and retrospective detection (fixed effects $\left.d=0.84\right), Q(2)=$ $7.98, p=.018, I^{2}=74.94 \%$. Hence, it is unlikely that the decreased detection rate for the longer than the shorted delays is related to the fact that we changed one of the two pilot details in Experiment 3. 
was again high (98.8\%) with only one incorrect instance. This referred to an incidental detail and manipulation was detected retrospectively. These findings suggest that the ability to detect manipulations was not confined by the ability to accurately reproduce the witnessed details. Although these results are consistent with earlier work on eyewitness identification decisions (Chapters 3 \& 9; Sagana et al., 2014a; Sagana et al., 2014b), interpretations should be cautious given the small number of inaccurately recalled details.

\section{The Effect of Detail Importance on Detection}

\section{Experiments 1 and 2}

To examine the impact of detail relevance on concurrent and retrospective detection rates, we applied a regression methodology known as Generalized Estimating Equations (GEE) analysis. This method enables incorporating repeated observations for all performed manipulations, leading to a total 190 observations. Of these, 115 referred to plot and 75 referred to incidental details.

Relevance predicted concurrent, Wald $\chi^{2}(1, N=190)=13.00, p<.001, b=-1.25$, and retrospective detection, Wald $\chi^{2}(1, N=190)=12.65, p<.001, b=-1.86$. In line with our hypothesis, manipulations of plot details were more likely to be detected than those of incidental details. Figure 7.1 displays the proportion of concurrent and retrospective detections for plot and incidental details across all three experiments.

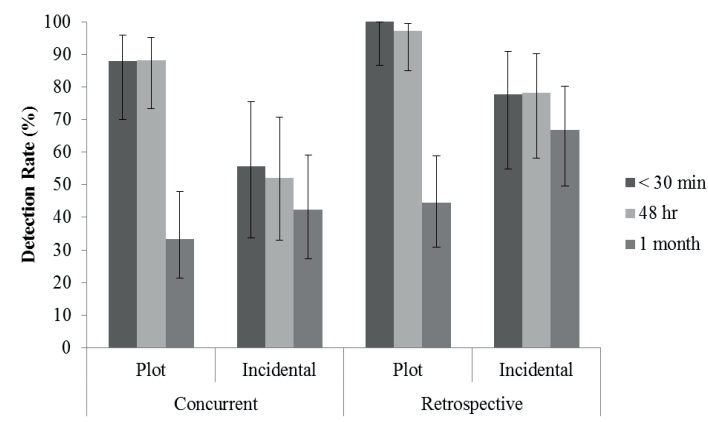

Figure 7.1 | Proportion of concurrent and retrospective detection for details concerning plot and incidental details as a function of delay. Error bars represent $95 \%$ confidence intervals $(95 \% \mathrm{Cl})$.

\section{Experiment 3}

Likewise, we examined the impact of detail relevance (plot vs. incidental) on concurrent and retrospective detection. Of the 78 observations, 45 referred to plot and 33 referred to incidental details. However, the effect of detail relevance was non-signif- 
icant for concurrent, Wald $\chi^{2}(1, N=78) \leq 0.67, p=.413, b=0.39$, and retrospective detection, Wald $\chi^{2}(1, N=78) \leq 3.71, p=.054, b=0.92$. In contrast to our predictions, detail relevance did not affect detection rates for the longer retention interval.

\section{DISCUSSION}

In a series of three studies we examined the ability of eyewitnesses to detect manipulations introduced to their testimony. We showed that eyewitnesses frequently miss manipulations in the specifics of a statement that they had written at an earlier stage. As expected, the magnitude of the effect was larger for longer (1 month) than for shorter delays (minutes or $48 \mathrm{hr}$ ). Indeed, for the longer delay, the results were most striking with only $36-52 \%$ of the manipulations being detected, compared with the $74-89 \%$ detections with shorter delays. Taken together, these results suggest that a substantial proportion of manipulations in eyewitnesses' written statements went undetected even minutes after giving testimony. Additionally, the overall high levels of recall accuracy (97.3-98.7\%) for the to-be manipulated details imply that even information that has been encoded and recalled accurately is susceptible to covert manipulations.

Our results are reminiscent of studies on autobiographical accuracy in the context of diary entries about everyday activities. These studies observed that many participants are willing to accept manipulated fragments inserted in their diaries as genuine memories (Barclay \& Wellman, 1986; Merckelbach et al., 1997). Nevertheless, the eyewitness context is different from diary entries of ordinary though memorable activities. The witnessed event is often outstanding and also associated with heightened levels of stress and arousal. These may affect eyewitness testimony (Christianson, 1992; Deffenbacher et al., 2004; Smeets et al., 2009). The current results refer to that setting. Our findings are also consistent with recent studies indicating that eyewitnesses can be unaware of covert changes in the outcome of their identification decisions (Chapters 3 \& 4; Sagana et al., 2013; 2014b). Additionally, they replicate Christianson et al. (Experiment 1 ; 2007) who also found that witnesses accept unreported details as their own testimony, even when they are requested to search actively for errors. Our findings expand Christianson et al.'s (2007) work in that they demonstrate that although writing allows for strong monitoring and control of the produced information (Grabowski, 2007), this may not be enough for filtering out manipulations or errors in one's statement. Moreover, our findings are in line with studies on the misinformation effect, which indicate increased acceptance of misinformation for longer compared with shorter retention intervals (Belli et al., 1992; Frost et al., 2002; Higham, 1998; Loftus, 2005; Underwood \& Pezdek, 1998). 
Drawing from the attentional narrowing hypothesis, we expected that encoding of essential information should be stronger than encoding of trivial information (Christianson, 1992; Christianson \& Loftus, 1987, 1991). Accordingly, we predicted higher detection rates for plot than incidental detail manipulations. Our hypothesis was supported for the shorter but not for the longer retention interval. However, given the ceiling effect of accuracy for both plot (Experiments 1/2: 99.1\%; Experiment 3: $100 \%$ ) and incidental to-be-manipulated details (Experiments 1/2: 94.7\%; Experiment 3: $97.0 \%)$, it is unlikely that the difference in detection rates for the shorter interval was caused by superior encoding. Instead, details that are essential for unfolding the story seem to be better preserved in memory, resulting in higher detection for plot than incidental detail manipulations. For example, earlier findings indicate that the negative influence of newly acquired information on memory is weaker for details with strong causal and temporal connections (Migueles \& García-Bajos, 2007; Myers, O'Brien, Balota, \& Toyofuku, 1984). Relatedly, the longer delay might have disrupted the memory of the event and the narrative might have become disintegrated. As a result the advantage in detection of plot than incidental detail manipulations should have been compromised. Nevertheless, the variation in detection rates within the plot, and to a lesser extent, incidental details indicates that additional factors may moderate the effect of blindness. Indeed, details virtually never differ only in one aspect. Details differ in valence, memorability, semantic relatedness, all of which can influence memory (Dolcos, LaBar, \& Cabeza, 2005; Jacques \& Levine, 2007; LaBar \& Cabeza, 2006; Talmi \& Moscovitch, 2004). However, we did not match the items on factors such as valence or semantic relatedness. Therefore, based on the present findings, we cannot draw firm conclusions about the effect of detail relevance. Still, they suggest that this topic warrants more systematic research.

Our findings are relevant for law enforcement agencies and legal professionals. Mistaken or deliberate manipulations even of minor details can impede the accurate reconstruction of an event and possibly incriminate innocent suspects. Importantly, the present work speaks to the risk of long delays between police investigation and court trials. Considering that even under the speedy trial act as applied in the US judicial system, trials begin the earliest 90-120 days after the arrest of the suspect (Shermer et al., 2011), the increasing acceptance of manipulations with longer delays is worrying. The current findings suggest that by the time witnesses would be crossexamined in court, a large proportion of witnesses would fail to realise that their statement contains mistakes. The issues emerging from our line of work directly appeal to the importance for triers of fact to check for the consistency between witness statements and video recordings of witness interviews.

The limitations of the present studies have to be acknowledged. First, our a priori decision about which details to alter was based on pilot work that informed us on 
the frequency with which each detail was reported. Still, we could only perform $60 \%$ of planned manipulations on average,. This loss of data undermined our statistical power, posing restrictions to the complexity of our analyses. Second, we failed to find evidence for differential encoding. We believe that the restricted complexity of the film may account for this outcome. Specifically, the film has a simple plot and no parallel action. Thus, participants may not have been challenged on whether they should allocate their attention to plot or incidental details. The results are still informative as they denote that weak encoding is not a sine qua non for the failure to detect manipulations. Nonetheless, examining differential encoding as a moderator may yield some interesting insights for the underpinnings of the effect. Third, defining what makes a detail relevant is highly subjective. Indeed, had we used a different method of determining relevance, the pattern of results might have been different. In the present study, relevance was defined based on how essential the detail was for the course of the events (e.g., Dalton \& Daneman, 2006; Wright \& Stroud, 1998) and on how frequently those details were reported in free recall on a pilot study (e.g., Heath \& Erickson, 1998; Saunders, 2009). Hence, we believe this hybrid definition to be the least biased and therefore suitable for this line of research.

In conclusion, in a series of three experiments we showed that eyewitnesses can be unaware of errors introduced in their written statements, with the effect being more pronounced for longer than shorter delays. Future research should look systematically into the factors that underlie poor detection of manipulations. The current findings, along with earlier work on the fallibility of autobiographical memory (Barclay \& Wellman, 1986; Merckelbach et al., 1997), show how easy it is to compromise the quality of eyewitness statements, and call for further research in this area. 



\section{PART 4}

\section{UNRAVELLING THE MECHANISM}





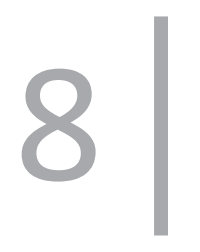

Self-relevance does not moderate choice blindness in adolescents and children 


\section{ABSTRACT}

In two experiments we tested the choice blindness in adolescents aged 11-16 years (Experiment $1, N=87$ ) and children aged $7-10$ years (Experiment 2, $N=$ 117) for the first time. Analogous to previously reported findings with adult participants, we expected to replicate the robust effect in these age groups. Furthermore, we investigated the hypothesis that self-relevance of choices, defined as the extent to which the self is implicated in a choice, moderates the choice blindness effect in adolescents and children. To this end, we directly compared high and low self-relevance conditions. As expected, the choice blindness effect was robust across age groups. Little support was found for the idea that self-relevance moderates the choice blindness effect. Specifically, no effect of self-relevance on choice blindness was found in adolescents, while the findings in the child sample were inconsistent. Different possible interpretations of the results as well as the possible role of ambiguity for the choice blindness effect are discussed. 


\section{INTRODUCTION}

Our life is replete with making choices. Each day we have to decide what to wear, what to eat, or more importantly, which doctor to visit. Although most of us would agree that we are aware of those choices and that we can easily detect a mismatch between our choice and the obtained result, recent research indicates quite the opposite. Rather, this research shows that people frequently fail to detect a mismatch between their choice and the achieved outcome when their choice is secretly manipulated. This effect is known as choice blindness.

In the first seminal experiment on choice blindness, participants had to decide which of two female faces they found more attractive (Johansson et al., 2005). Following their decision, participants were handed the selected face and asked to motivate their decision. In three of the 15 trials, however, their choice was manipulated by means of a magical card trick, so that participants ended up with the non-selected face. The findings were striking: The overwhelming majority (87\%) of the manipulated trials went undetected at the time of the manipulation (i.e., concurrently). After the end of the experiment $70 \%$ of the manipulations remained undetected (i.e., retrospectively). Paradoxically, some of the participants included facial features in their motivations that did not correspond to the picture they held in their hands. Conversely, some referred to features that were true only for the manipulated outcome and could not account for the initial choice.

More recent studies investigating choice blindness picked up on the critique that ratings of facial attractiveness lack personal relevance to the participants (Moore \& Haggard, 2006). Conversely to the criticism, this research showed that participants can be blind to manipulations of their moral attitudes (Hall et al., 2012), political attitudes (Hall et al., 2013), and reports concerning their history of norm-violating behaviours (Sauerland, Schell, et al., 2013). However, none of these studies provided a direct comparison between more and less self-relevant conditions. Furthermore, the performed choices had no consequences.

A large body of literature on the so-called self-reference effect in memory predicts that detection rates should differ as a function of the level of self-relevance of the task. The effect was first demonstrated and defined by Rogers, Kuiper, and Kirker (1977) as the tendency of people to encode information in which the self is implicated in a privileged way. The term self-reference has often been used synonymous with personal relevance or self-relevance in the literature (Bargh, 1982; Block, Greenberg, \& Goodman, 2009; Panayiotou \& Vrana, 2004) and we will use the latter terms throughout this chapter. The self-relevance effect has been studied in dozens of research papers and demonstrated its robustness across different age groups, stimuli and stimulus presentations as well as encoding and recall conditions (Symons \& Johnson, 1997). 
To name just a few examples, memory for self-relevant terms is superior to memory for non-self-relevant terms, as are self-relevant encoding strategies compared with semantic and other relevant encoding strategies (Forsyth \& Wibberly, 1993; Rogers et al., 1977; Zhu et al., 2012). Likewise, flashbulb memories for self-relevant events have been shown to be stronger than for non-self-relevant events (Brown \& Kulik, 1977); and mock jurors demonstrated better recall and recognition performance for a selfrelevant crime (on campus rape), compared with a non-self-relevant crime (prison rape; Block et al., 2009). The first aim of the current paper was to test whether the choice blindness effect depends on this self-relevance effect. Based on the literature our expectation was that high self-relevance decisions should lead to higher detection rates than low self-relevance decisions.

Our second aim was to extend the choice blindness paradigm to adolescent and child populations. Although the choice blindness effect has been substantiated in a variety of settings (e.g., personal interactions and computer based tasks; in labs, in a supermarket, in a university library) and for a variety of stimuli (visual, auditory, olfactory, gustatory; Hall et al., 2010; Johansson et al., 2008; Johansson et al., 2005; Chapter 2; Sauerland, Sagana, et al., 2013), it is imperative to assess whether it will also appear in non-adult populations in order to demonstrate and fully understand the robustness of this effect. Drawing from studies that show that children are less likely to detect changes in an environment than adults (e.g., Fletcher-Watson, Collis, Findlay, \& Leekam, 2009; Shore, Burack, Miller, Joseph, \& Enns, 2006), one might expect detection rates to be smaller in children compared with adults.

This prediction dovetails nicely with developmental work on compliance revealing that young children are more likely to be persuaded by external influences relative to older children and adults (Ceci \& Bruck, 1993). The reason is that memory encoding is less well-developed in young children making them more reliant on others than older children and adults. Based on this work, one might argue that choice blindness manipulations are more likely to have an impact in younger than older children leading to heightened choice blindness effects and smaller detection rates. Indeed, when drawing parallels with developmental work on source monitoring, research clearly suggests that children have more difficulties with monitoring the sources of their memories than adults especially when the sources are similar (Lindsay, Johnson, \& Kwon, 1991). In a sense, participants are involved in source monitoring during a choice blindness experiment. That is, participants have to decide whether the manipulated choice is their own choice and recollection or whether it is provided by someone else. Children have more problems with making such memorial decisions rendering them more prone to choice blindness than adults. Furthermore, based on the finding that the self-relevance effect occurs as early as age four to six (Cunningham, Vergunst, Macrae, \& Turk, 2013; Sui \& Zhu, 2005), we predicted that the moderating effect 
of self-relevance on choice blindness should be present across different age groups, with all age groups displaying less blindness effects in high compared with low selfrelevance conditions.

To assess our hypotheses, we tested adolescents (11-16 years; Experiment 1 ) and children (7-10 years; Experiment 2) and subjected them to two different choice blindness paradigms, using stimuli and procedures that were suited for each age group. Although the variation in stimuli and procedures puts restrictions on comparisons across experiments, these modifications were crucial in order to generate valid high and low self-relevance conditions for the different age samples. Note that, had we used the same task across different age groups, it is likely that these would have differed in terms of how they interpreted the task. To specify the experimental procedures, adolescents (Experiment 1) either indicated their preference regarding five interventions (e.g., new classroom chairs, black boards, vending machines) that were planned to take place in their school environment (high self-relevance), or provided more general estimations (e.g., estimate which of two items was more expensive; low self-relevance). We selected the school environment as a source for our manipulations in this experiment because school life constitutes a fundamental part of adolescents' and children's everyday life. Accordingly, changes in the school environment should generally be of interest to the students. Children (Experiment 2), on the other hand, made five preferential choices regarding two toys (high self-relevance), or made an objective assessment (i.e., which of two animals was faster or which of two foods was sweeter; low self-relevance). This setup was chosen to address children's difficulties in thinking in abstract ways.

Additionally, we explored the tendency to act according to social demands as a possible moderator of the choice blindness effect. Indeed, it is possible that blindness to manipulations is associated with construct of social desirability, with people failing to report that they did notice the change in manipulated trials due to their need for approval from the experimenter (Crowne \& Marlowe, 1960).

\section{Ethics Statement}

The studies reported in this paper were approved by the standing ethical committee of the Faculty of Psychology and Neuroscience (Ethics Committee Psychology, ECP). Written consent was obtained for Experiment 1. In line with the formal requirements as dictated by the ECP for testing minors, oral participant consent was obtained for Experiment 2 and parental written consent for both Experiments 1 and 2. 


\section{EXPERIMENT 1}

\section{Method}

\section{Participants}

Participants were eighty-seven German high-school students from one high-school (34 boys; $M_{\text {age }}=14.1$ years, $S D_{\text {age }}=1.4$, age range: $11-16$ years). They provided parental consent forms prior to participation that was on voluntary basis; no monetary awards or course credits were awarded.

\section{Self-relevance manipulation}

In the high self-relevance condition, participants made choices that referred to changes in their school environment (i.e., new classroom chairs, classroom black boards, etc.). In the low self-relevance condition, the choices referred to the same objects, but no association with participants' high-school was established.

\section{Design}

A 2 (self-relevance: high vs. low) x 2 (similarity: high vs. low) between-subjects design was employed. Participants were randomly assigned to one of the four experimental groups. The distribution of participants across conditions was 22 (low self-relevance) low similarity), 23 (low/high), 20 (high/low), and 22 (high/high).

\section{Independent Variables Concurrent and Retrospective Detection}

Across the two experiments reported in this paper, the concurrent and retrospective detection rates served as the dependent variables. Concurrent detection refers to detection immediately after the presentation of the manipulated outcome. In the computer-based Experiment 1, this refers to comments provided on a piece of paper intended for participants to write down the reasons for their selection after each trial. In Experiment 2, where a direct interaction took place between experimenter and child participants, concurrent detection was inferred when children told the experimenter that they had chosen the other toy or that she had made a mistake. Retrospective detection refers to detections that were documented by means of the post-test questionnaire (Experiment 1) or post-test interview (Experiment 2). If, however, participants indicated manipulations that had not occurred, no retrospective detection was assumed.

\section{Stimulus selection}

Self-relevance. To ensure that the required choices were self-relevant to high-school students, we conducted a first pilot study, using a survey that consisted of eight ques- 
tions. These referred to the introduction of a new school logo, classroom wall colour, classroom chairs, classroom black boards, school lockers, school plants, and school vending machines. Participants indicated how relevant the indicated changes would be to them on a scale ranging from 1 (not important to me) to 10 (very important to $m e)$. Note that we referred to importance here, instead of self-relevance, as this term is easier to understand for high-school students. The following is an example item:

\begin{abstract}
"Your school wants to introduce a day on which all students have to wear the same t-shirt in order to promote student team spirit. How important would the design of this t-shirt be to you?"
\end{abstract}

Fifty-eight high-school students (12 boys; $M_{\text {age }}=15.4$ years, $S D_{\text {age }}=0.8$, age range: 13-16 years) were recruited through a link to the survey on social media. The average rated importance ranged from $M=4.74$ (school plants) to $M=7.24$ (classroom chairs). We selected the five interventions that were rated most relevant to the high-school students as stimulus categories. These included classroom chairs $(M=7.24, S D=2.47)$, school t-shirts $(M=6.76, S D=2.94)$, school vending machines $(M=6.59, S D=3.08)$, classroom wall colour $(M=5.40, S D=2.79)$, and classroom black boards $(M=5.26, S D$ $=2.84$ ). The intervention with the highest ratings (new classroom chairs) was selected as the choice to be made during the manipulated trial. This was to ensure that the manipulated trial constituted an item that was highly self-relevant to the participants.

Similarity. Two sets of stimuli were created, one with high and one with low similarity stimulus pairs. Specifically, we collected six pictures of different exemplars of each of the five selected stimulus categories (i.e., chairs, black boards, etc.) from search engines on the internet and company websites. We paired all six pictures of one category with each other (i.e., all chairs with other) and presented them to 60 second year psychology students (16 men; $19-51$ years, $M_{\text {age }}=21.7$ years, $S D_{\text {age }}=4.6$ ) who participated in exchange for course credit. Their task was to rate the similarity of each stimulus pair on a scale from 1 (very different) to 10 (very similar). For each of the five

Table 8.1 | Mean Similarity of the Selected Stimulus Pairs (Experiment 1).

\begin{tabular}{lcccc}
\hline & \multicolumn{3}{c}{ Similarity } \\
\cline { 2 - 5 } & \multicolumn{3}{c}{ Low } & High \\
\cline { 2 - 5 } & $M$ & $S D$ & $M$ & $S D$ \\
\hline Classroom wall colour & 3.22 & 1.84 & 6.43 & 2.43 \\
\hline Classroom black boards & 3.55 & 1.66 & 5.58 & 2.00 \\
\hline Classroom chairs & 4.13 & 1.88 & 8.07 & 1.36 \\
\hline School vending machines & 4.38 & 2.06 & 6.10 & 2.05 \\
\hline School t-shirts & 4.65 & 2.01 & 6.98 & 1.71 \\
\hline
\end{tabular}


stimulus categories, we selected those pairs that were rated most and least similar for our high and low similarity conditions. The difference between high and low similarity stimulus pairs was significant for all stimulus categories, $t s(59) \geq 7.11, p s<.001$. The means can be found in Table 8.1.

\section{Post-test questionnaire}

The post-test questionnaire was designed to examine whether participants had noticed our manipulations and refrained from revealing this and was used as a means to establish retrospective detection. First, participants were asked if they had any comments, suggestions or if they had had any problems, and if so what the nature of these problems was. Subsequently, participants were misled to believe that the experiment had employed two conditions: one in which some of their choices had been manipulated and one where this was not the case. Participant then had to indicate which condition they thought they had been assigned to. Those who indicated that they had been in the manipulated condition next specified how many manipulations they had noticed and for which specific picture pairs.

Social Desirability Scale-17 (SDS-17)

The SDS-17 (Stöber, 1999) contains 17 true/false self-report items that assess an individual's need for approval. The rationale of the instrument is that persons with higher need for approval tend to give more socially desirable responses than the average.

\section{Procedure}

Participants with parental approval were individually taken out of their class and brought to one of three computers. In the low self-relevance condition, students were told that the experimenters were interested in decision-making and decision outcomes as a cover story. These participants were asked about their preference regarding two objects (i.e., two chairs/black boards/ t-shirts etc.). However, no relation with their own school was established. In the high self-relevance condition, students were additionally told that the research group collaborated with the headmaster of their school. Specifically, participants were led to believe that their headmaster was interested in the opinion of the students because he wanted to implement some interventions at their school. Therefore, the students should carefully consider their choices because these would have an effect on the pending decisions. Before proceeding, the experimenters made sure that the participants understood the importance of these choices and their impact on the future.

After signing the informed consent form, the computer based experiment (Forster \& Forster, 2003) started with a practice trial. Participants were shown two objects and had to indicate their preference by pressing the 1 (left object) or 2 key (right 
object). No time limit was imposed on participants' decision time. Following the decision, a 200 ms mask appeared. Next the selected item reappeared and participants were asked to motivate their choice on a sheet of paper. After the example trial, the five analogous experimental trials followed. In the fourth trial (chairs), however, participants were not presented with their choice, but with the chair they had actually not selected. A demonstration of the procedure can be found in Figure 8.1. After participants were finished with the computer task, they filled in the SDS-17 and the post-test questionnaire. Finally, participants were asked not to talk about the study until it was finished, were offered candy, and thanked for participating. The debriefing took place after termination of data collection.

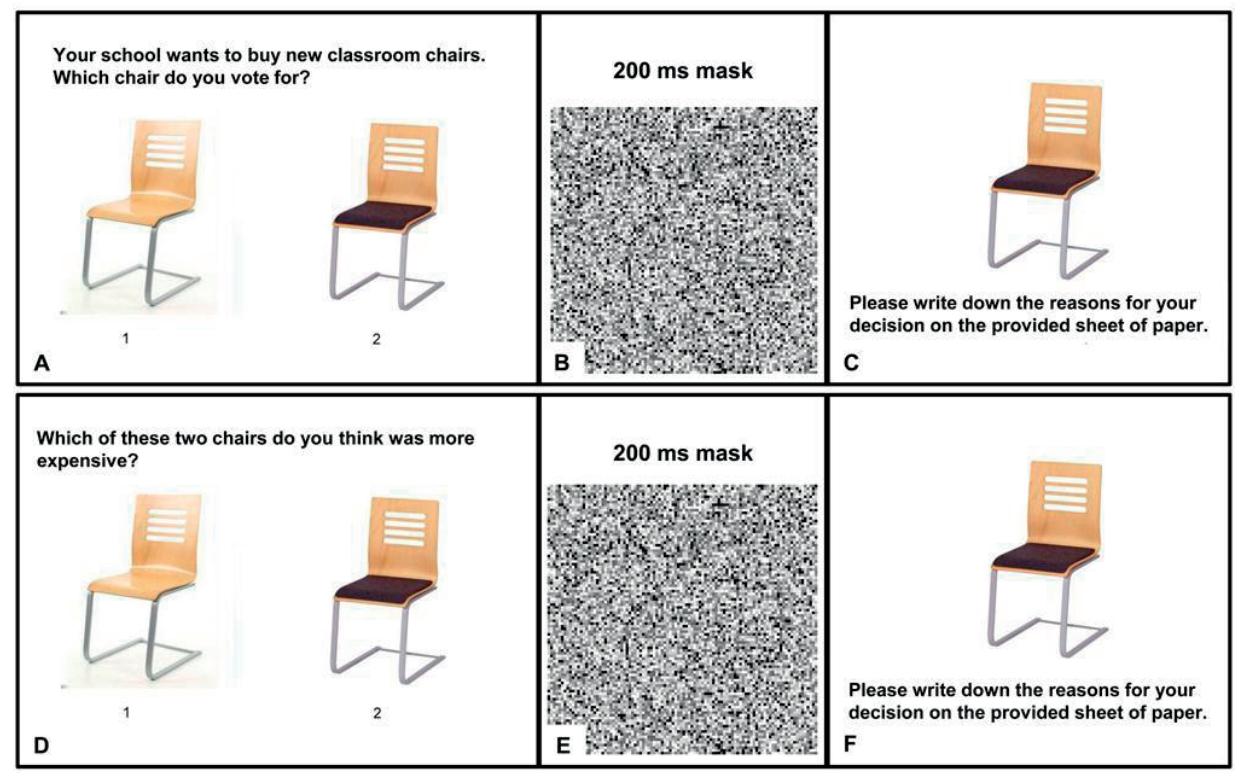

Figure 8.1 | Demonstration of the procedure of a high (A-C) and a low self-relevance (D-F) trial in Experiment 1.

A. Participants view two stimuli and indicate their choice by selecting 1 or 2 . No time limit is imposed. B. A masking pattern is presented for 200 ms. C. Participants' choice reappears and participants motivate their choice on a separate piece of paper. In a manipulated trial, participants' non-choice appears and participants are also asked to indicate motivate their "choice". D-F. Analogous procedure for a low self-relevance trial. 


\section{Results and Discussion}

\section{Choice Blindness}

The detection rates and 95\% confidence intervals for high and low self-relevance conditions across both experiments can be found in Table 8.2. Concurrent detection in the high self-relevance condition was $11.1 \%$, and $9.5 \%$ in the low self-relevance condition. Retrospective detection rates were $57.8 \%$ and $54.8 \%$, respectively.

\section{Effect of age}

To test the effect of age on blindness rates, we split the participants into two age groups (11-13 years vs. 14-16 years). The results showed that neither measure of detection varied as a function of age (concurrent detection: $\chi^{2}(1, N=87)=1.95, p=$ .222 , phi $=-.15$, retrospective detection: $\chi^{2}(1, N=87)=0.49, p=.620$, phi $\left.=.07\right)$.

Table 8.2 | Concurrent and Retrospective Detection Rates (\%) and 95\% Cls across Two Experiments.

\begin{tabular}{|c|c|c|c|c|c|c|}
\hline & & & \multicolumn{4}{|c|}{ Self-relevance } \\
\hline & & & High & Low & High & Low \\
\hline \multirow{2}{*}{$\begin{array}{l}\text { Experiment } \\
1\end{array}$} & \multirow{2}{*}{$\begin{array}{l}N \\
87\end{array}$} & \multirow{2}{*}{$\begin{array}{l}\text { Sample } \\
\text { Adolescents }\end{array}$} & \multicolumn{2}{|c|}{$\begin{array}{l}\text { Proportion concurrent detection (and } \\
\qquad 95 \% \mathrm{Cl} \text { ) }\end{array}$} & \multicolumn{2}{|c|}{$\begin{array}{l}\text { Proportion retrospective detection } \\
\qquad(\text { and } 95 \% \mathrm{Cl})\end{array}$} \\
\hline & & & $11.1(1.6 ; 20.7)$ & $9.5(0.2 ; 18.8)$ & $57.8(42.8 ; 72.8)$ & $54.8(39.1 ; 70.0)$ \\
\hline \multirow{2}{*}{2 (Toys) } & 55 & Younger children & $50.9(37.3 ; 64.5)$ & $50.9(37.3 ; 64.5)$ & $54.4(41.0 ; 68.1)$ & $52.7(39.1 ; 66.3)$ \\
\hline & 62 & Older children & $62.9(50.5 ; 75.3)$ & $51.6(38.8 ; 64.4)$ & $72.6(61.2 ; 84.0)$ & $56.5(43.8 ; 69.1)$ \\
\hline \multirow{2}{*}{2 (Erasers) } & 55 & Younger children & $56.3(28.9 ; 83.6)$ & $30.8(15.6 ; 45.9)$ & \multirow{2}{*}{\multicolumn{2}{|c|}{ not measured }} \\
\hline & 62 & Older children & $50.0(32.8 ; 67.2)$ & $65.4(45.8 ; 85.0)$ & & \\
\hline
\end{tabular}

$\mathrm{Cl}=$ confidence interval

\section{Effects of self-relevance and similarity}

Two logistic regression analyses were conducted to establish the effect of selfrelevance (high vs. low) and similarity (high vs. low) on concurrent and retrospective detection (yes vs. no). The interactions were non-significant (concurrent detection: Wald $\chi^{2}(1, N=87)=2.59, p=.108$; Nagelkerke's $R^{2}=.08$; retrospective detection: Wald $\chi^{2}(1, N=87)=0.98, p=.322$; Nagelkerke's $\left.R^{2}=.02\right)$. Models including only the main effects likewise yielded no significant results (concurrent detection: Wald $\chi^{2} s(1$, $N=87) \leq 0.21, p s \geq .648$; Nagelkerke's $R^{2} s=.01$; retrospective detection: Wald $\chi^{2} s(1$, $N=87) \leq 0.52, p s \geq .472$; Nagelkerke's $R^{2} s=.01$ ). The results for similarity concur with earlier findings (Johansson et al., 2005; Chapter 4; Sagana et al., 2013). Others, however, reported an effect of similarity for a limited selection of stimuli (Hall et al., 2010) or for concurrent (but not retrospective) detection (Chapter 2; Sauerland, Sagana, et al., 2013). 
Relationship of Choice Blindness and Social Desirability

In accordance with most earlier findings on the relationship between choice blindness and social desirability (Chapter 2; Sauerland, Sagana, et al., 2013; Sauerland, Schell, et al., 2013, Experiment 1 and 2), no significant correlations were obtained for both measures of detection (concurrent detection: $r(85)=.12, p=.265$; retrospective detection: $r(85)=.14, p=.185)$. This deems the idea that choice blindness is merely an effect of participants' tendency to comply with the presumed expectations of the experimenter or the tendency to act according to social demands unlikely.

\section{EXPERIMENT 2}

A moderating effect of self-relevance on the choice blindness effect was absent in adolescent high-school students (Experiment 1). In Experiment 2, we tested whether self-relevance would moderate the choice blindness effect in children at the elementary school level.

\section{Method}

\section{Participants}

Participants were $N=117$ children (51 boys, $M_{\text {age }}=8.56, S D_{\text {age }}=1.07$, age range 7-10 years) from three primary schools. Parental consent was obtained prior to participation and testing permission was granted from each school individually. Oral consent was obtained from the child participants.

\section{Self-relevance manipulation}

Self-relevance was manipulated by varying question type. In high self-relevance trials, children had to indicate a preference. Specifically, they were asked which of two stimuli they liked better ("Which animal [food] do you like better?"). In the low selfrelevance condition, the question referred to an objective feature of the animal or the food stimulus ("Which animal is faster?" or "Which food is sweeter?").

\section{Design}

Self-relevance was once manipulated within-subjects (plastic toys) and once betweensubjects (erasers). For the (within-subjects) toy manipulation, the order of presentation (high-low self-relevance vs. low-high self-relevance) as well as the stimulus set used for each condition was counterbalanced between-subjects. There was no effect of these two variables on the dependent variables. Table 8.4 gives an overview of the eight conditions used. For the (between-subjects) eraser manipulation, the stimulus 
set used (eraser animals vs. eraser foods) was counterbalanced between-subjects next to self-relevance. Preferably, we would have asked child participants which of our two tasks they found more relevant to themselves (indication of preference vs. assessment of fastness/sweetness). However, this did not seem to age appropriate. Alternatively and in line with literature demonstrating that stronger attitudes can be evidenced in faster responses (Bassili, 1996; Bohner \& Dickel, 2011), we measured child participants' response times. We expected response times for high-relevance conditions to be faster than for low self-relevance conditions.

\section{Materials}

Three black carton boxes $(38.0 \mathrm{~cm} \times 19.5 \mathrm{~cm} \times 26.5 \mathrm{~cm}$ ) with an open back and two openings on the top were used to covertly perform the manipulations. The open back allowed the experimenters to see the stimuli; the two openings enabled them to retrieve the stimuli during the experiment. The front side of the boxes (i.e., the side facing the child), displayed the numbers 1 and 2, along with a white line in the middle, indicating two compartments, although there was no actual separator inside the box. Usage of these boxes was practiced with all experimenters prior to testing.

Stimulus selection. Two pairs of plastic animals, two pairs of plastic foods toys and a variety of erasers representing animals or foods were used as stimuli. The four plastic toy stimulus pairs were matched in terms of average preference within each stimulus pair, as established in pilot work with 20 child participants ( 2 boys; $M_{\text {age }}=8.35, S D_{\text {age }}$ = 1.14, age range: 7-10) with written parental consent and oral participant consent. Table 8.3 presents the mean ranking for each stimulus. The pairs selected for the study were: lion - tiger (wild animals), zebra - horse (hoofed animals), grapes - watermelon (fruits), and chocolate bar - pudding (sweets). According to Wilcoxon signed ranked tests, none of the pairs differed significantly from each other in terms of preference or fastness/sweetness ranking, all $|z| s \leq 1.65, p s \geq .098$.

Table 8.3 | Mean Ranking Order and Standard Deviation for Plastic Animals and Foods (Experiment 2).

\begin{tabular}{lcccc}
\hline & \multicolumn{2}{c}{ High self-relevance question } & \multicolumn{2}{c}{ Low self-relevance question } \\
\hline Stimuli & $M$ & $S D$ & $M$ & $S D$ \\
\hline Lion & 3.15 & 1.39 & 2.15 & 1.18 \\
\hline Tiger & 2.80 & 1.28 & 1.70 & 0.97 \\
\hline Zebra & 2.35 & 1.22 & 2.05 & 0.68 \\
\hline Horse & 2.10 & 1.37 & 1.70 & 1.26 \\
\hline Grapes & 2.60 & 1.27 & 2.60 & 1.14 \\
\hline Watermelon & 2.85 & 1.84 & 2.60 & 1.31 \\
\hline Chocolate Bar & 3.45 & 1.35 & 3.45 & 1.35 \\
\hline Pudding & 3.60 & 1.35 & 3.65 & 1.26 \\
\hline
\end{tabular}


The pilot participants also ranked two sets of erasers (animals and foods). Based on the results, different combinations of erasers were used for the high and low selfrelevance conditions. While we had planned to only use pairs that were similar in their rankings, this did not always work out when testing in the field. Pairings that significantly differed in their rankings were used in 28 of the cases $(23.9 \%)$. In order to prevent that a possible effect of self-relevance would be masked by this, we reran the eraser analyses excluding all trials in which an eraser pair had been used that differed significantly in the rankings. The pattern of results remained identical. Therefore, we report the results for the full sample in the results section.

\section{Procedure}

In order to create a test setting appropriate for children, this experiment did not employ a computer-based task, but included a direct interaction between participant and experimenter. Previous research has shown that choice blindness effects occur in both settings (Johansson et al., 2008).

In their classrooms, participants were told that they could take part in a game in which they would answer questions about different toys. Those children who wanted to participate were then individually led to a different room where two of the six experimenters awaited them. The experiment started with an alleged practice trial containing a pair of foods or animal erasers. Note that the children would receive the eraser they had not chosen as a small gift following their participation. This manipulation was conducted to see if children would notice a manipulation after a short delay. After this alleged practice trial, four analogous trials followed. No time limit was imposed on children's decision time.

In each trial, the experimenter placed a pair of stimuli into the black box. She then reached through the openings on top and presented the two stimuli to the child. Depending on the condition, the experimenter asked a high or low self-relevance
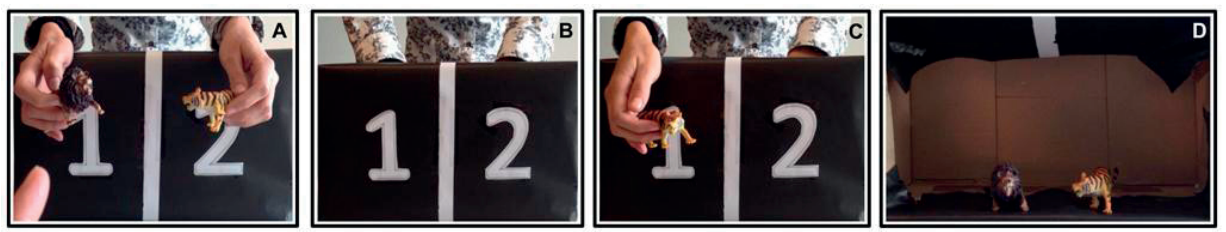

Figure 8.2 | Demonstration of the procedure of a manipulated trial of Experiment 2.

A. Participants select one of the two presented toys, deciding either which one they like better (high self-relevance) or which of the two animals is faster (low self-relevance). No time limit is imposed. B. The experimenter puts both toys back into the box through the two openings on the top and switches the two toys in her hands for this manipulated trial. C. The experimenter extracts the non-selected toy from the opening which previously seemed to contain the selected toy. D. Rear view of the used box. The absence of a division within the box allows for a switch of the different toys in manipulated trials. 
Table 8.4 | Overview of the Eight Different Conditions and the Procedure in Experiment 2.

\begin{tabular}{|c|c|c|c|c|c|c|c|c|}
\hline Condition & 1 & 2 & 3 & 4 & 5 & 6 & 7 & 8 \\
\hline Eraser trial (a) & Animal* & Animal* & Food* & Food* & Animal & Animal & Food & Food \\
\hline Plastic toy trial $1^{\dagger}$ & Hoofed* & Wildlife* & Fruit* & Sweet* & Hoofed & Wildlife & Fruit & Sweet \\
\hline Plastic toy trial 2 & Wildlife & Hoofed & Sweet & Fruit & Wildlife* & Hoofed* & Sweet* & Fruit* \\
\hline Plastic toy trial 3 & Fruit* & Sweet* & Hoofed* & Wildlife* & Fruit & Sweet & Hoofed & Wildlife \\
\hline Plastic toy trial $4^{\dagger}$ & Sweet & Fruit & Wildlife & Hoofed & Sweet* & Fruit* & Wildlife* & Hoofed* \\
\hline
\end{tabular}

Eraser trial (b): Receive non-selected object from eraser trial ${ }^{\dagger}$

Note: ${ }^{\dagger}=$ manipulated trial; ${ }^{*}=$ high relevance trial.

question (i.e., high self-relevance: "Which of the two do you like better?"; low selfrelevance: "Which of the two is faster?"; "Which of the two is sweeter?"). After participants made a decision, the experimenter put the two stimuli back into their corresponding openings. Subsequently, she took the selected stimulus out of the box again and asked the child why they had selected that eraser or plastic toy. The occlusion time was as long as it takes to put two stimuli back into a box and take one out again. Although we have not measured the duration, we assume that this part of the procedure took about 1-2 seconds. A trial was concluded by putting the selected stimulus back into the opening and removing both stimuli from the box via the backside. In preparation of the next trial, the experimenter put a new stimulus pair into the box. A demonstration of the procedure can be found in Figure 8.2. Table 8.4 illustrates the setup of the eight different administered conditions.

The manipulations were executed in plastic toy trials 1 and 4 . In these trials, the experimenter covertly switched the two stimuli inside of the box and retrieved the non-selected stimulus from the opening corresponding to the selected stimulus. If the child noticed the swap, the experimenter said that she must have made a mistake, retrieved the selected stimulus and asked why they had chosen that object. While one experimenter performed the experiment, a second experimenter measured the children's decision times, wrote down their selections, and documented manipulation detections.

After completion of the plastic toy trials, participants were asked if the experimenter made any mistakes during the experiment and if so during which trial. This post-test interview served as a means of measuring retrospective detection. Finally, participants were thanked for their participation and received the non-selected eraser from the eraser trial as a gift. If children at this point said that they had not chosen the eraser they were given as a present, this was counted as concurrent detection. Retrospective detection was not measured for the eraser trials since the manipulation occurred after the post-test interview, from which retrospective detection was 
inferred. Children were fully debriefed in their classrooms and a debriefing letter was provided for the parents.

\section{Results and Discussion}

\section{Manipulation check}

As expected, the response times averaged across the two high self-relevance plastic toy decisions were faster $(M=2.5 \mathrm{~s}, S D=1.74)$ than those for the two low selfrelevance plastic toy decisions $(M=3.4 \mathrm{~s}, S D=1.69), t(116)=-5.22, p<.001, d=$ -0.52 . Additionally, responses to the high self-relevance eraser trial were faster $(M=$ $2.3 \mathrm{~s}, S D=1.94)$ than to the low self-relevance eraser trial $(M=2.9 \mathrm{~s}, S D=1.84), t(115)$ $=-2.13, p=.036, d=-0.39$. Note that for the analyses of response times, inferential analyses were conducted on log-transformed data (i.e., log base 10) due to significant positive skewness and kurtosis in the response time distribution. The reported means are back-transformed values.

These findings support our idea that judgments about preferences were more selfrelevant to our child participants than objective assessments about speed or sweetness. Another possible interpretation of these results could be that the different tasks (semantic memory task vs. preferential task) simply require different amounts of processing time. Future studies could avoid this limitation by using identical tasks for high and low self-relevance conditions while varying self-relevance in terms of incentives (e.g., payment).

\section{Choice Blindness and Self-Relevance}

The concurrent detection rates in the high self-relevance condition were $57.3 \%$ (plastic toys) and $54.7 \%$ (erasers), and $51.3 \%$ (toys) and $47.9 \%$ (erasers) in the low self-relevance condition. Retrospective detection rates for the plastic toys were $54.7 \%$ (high self-relevance)and $47.9 \%$ (low self-relevance), respectively (Table 8.2 also includes the means for the two age groups 7-8 years vs. 9-10 years). Recall that retrospective detection was not measured for erasers.

Plastic toy manipulations. Given that a standard ordinary least square regression analysis would not be a valid statistical model for this design, which includes withinsubjects factors and therefore produces correlated data, we opted for Generalized Estimating Equations model (GEE). The GEE model provides an appropriate alternative, as it accounts for the correlated residuals via the specification of a working correlation matrix.

For the plastic toy manipulations, both age (7-8 years vs. 9-10 years) and selfrelevance were entered into a GEE analysis as predictors of detection. For concurrent detection, the effect of age was significant, $b=1.01, S E=.38$, Wald $\chi^{2}(1, N=117)=$ 
$6.98, p=.008$. Specifically, 7-8 year old children were less likely to detect the manipulation (44.5\%) than 9-10 year old children (62.9\%). The main effect of self-relevance as well as the interaction between both factors were non-significant, Wald $\chi^{2} s(1, N$ $=117) \leq 2.63, p s \geq .105$. A model containing only the main effects returned similar results.

For retrospective detection, the effect of age was also significant, $b=1.15, S E=$ .39 , Wald $\chi^{2}(1, N=117)=8.80, p=.003$, with older children displaying higher detection rates $(70.2 \%)$ than younger ones $(48.2 \%)$. The effect of self-relevance was nonsignificant, Wald $\chi^{2}(1, N=117)=2.31, p=.128$. A model containing only the main effects did reveal a significant main effect of self-relevance, $b=0.45, S E=.22$, Wald $\chi^{2}(1, N=117)=4.30, p=.038$. Specifically, high self-relevance manipulations were detected more often (65.0\%) than low self-relevance manipulations (54.7\%).

Eraser manipulations. For the eraser manipulations (between-subjects factor), a logistic regression analysis was conducted to examine the effect of self-relevance (high vs. low) and age (7-8 years vs. 9-10 years) on concurrent detection (yes vs. no). Both factors as well as the interaction were entered simultaneously. The effect of selfrelevance failed to reach significance, but indicated a trend, Wald $\chi^{2}(1, N=117)=3.54$, $p=.060$, as did the interaction between age and self-relevance, Wald $\chi^{2}(1, N=117)=$ 2.94, $p=.087$; Nagelkerke's $R^{2}=.08$. For exploratory reasons we performed post-hoc comparisons which showed that high-relevance manipulations tended to be detected more often (56.3\%) than low self-relevance manipulations $(30.8 \%)$ in younger, $\chi^{2}(1, N$ $=117)=3.42, p=.081$, phi $=-0.25$, but not older children, $\chi^{2}(1, N=117)=0.29, p=$ $.609, p h i=0.07$. A model including only the main effects did not reveal any significant effects, $p s \leq .101$. One may argue that detection rates for the eraser manipulations might have been deflated due to the fact that children received an eraser as a gift, that is, that children might have kept quiet about a detected manipulation out of fear that then they would not receive a gift at all. If this were the case, however, the detection rates for eraser trials should, on average, be lower than the detection rates for plastic toy manipulations. Inspection of Table 8.2, however, shows that this was not the case.

\section{GENERAL DISCUSSION}

Across two experiments, we sought to extend the existing literature on choice blindness in two ways. We aimed to examine the effect of self-relevance on choice blindness rates. Testing non-adult samples for the first time, this research question was implemented across two different age groups, namely in adolescents and children. We expected to replicate earlier findings demonstrating a strong choice blindness 
effect. Furthermore, we hypothesized that high self-relevance decisions would lead to higher detection rates than low self-relevance decisions across age groups. As such, our two experiments are the first published ones to provide a direct comparison between more and less self-relevant conditions. As predicted, a considerable proportion of participants were blind to our manipulations. Unexpectedly, however, our results do not support the idea that self-relevance can decrease blindness rates (Moore \& Haggard, 2006). In the following, we will address both of these findings in more detail.

In both experiments we found substantial choice blindness effects, with blindness rates ranging from $37 \%$ to $91 \%$ concurrently and $27 \%$ to $47 \%$ in retrospect. The variance across experiments is remarkable, with especially high blindness rates in Experiment 1. One explanation for this might be differences in the immediacy of the consequences following the choices in the high self-relevance conditions. Specifically, in Experiment 2, child participants had to choose between one of two toys, a task that is likely to be of immediate importance at that age. In Experiment 1, however, the consequences of the choices that participants made would only follow in the undefined future. The study of the strength of the choice blindness effect as a function of immediacy of decision consequences might be an interesting alley for future research.

Although we could not make direct comparisons across the two samples, because we had to use different tasks that were suited for both age groups, we were able to make comparisons within children and adolescents. These comparisons showed that children aged 7-8 years old were sometimes less likely to detect our manipulated trials than children aged 9-10 years. This effect only appeared for one (plastic toys), but not the other (eraser) manipulation. Furthermore, we found no effect of age on choice blindness rates within the adolescent sample. One reason for this age trend could be that younger children (aged 7-8) are more likely to be persuaded by external "suggestive" information than older children (9-10-year-olds; e.g., Bruck \& Ceci, 1999). The underlying rationale is two-fold. First of all, younger children's memory encoding is less well-developed than that of older children's. This entails that younger children are more likely to trust other people when talking about their own memories and choices. Relatedly, younger children are more likely to be influenced by authoritarian people (e.g., experimenters) relative to older children (Ceci \& Bruck, 1993). Combined with weaker encoding and source monitoring capacities (Lindsay et al., 1991), these mechanisms might have caused the differences in detection rates between the younger and older children that were found for some of our analyses.

Regarding the impact of self-relevance on choice blindness, our data provide only little evidence in support of the idea that self-relevance can decrease blindness rates: In the adolescent sample, no indication for such an effect was found. In the child sample, the findings were inconsistent. Specifically, there was a significant effect in the expected direction for retrospective, but not concurrent detection of the toy 
manipulation. Furthermore, for the eraser manipulation, we found a marginally significant effect for younger (7-8 years), but not for older children (9-10 years).

Three inferences are possible when looking at the results on the effect of selfrelevance on choice blindness: First, one may argue that self-relevance simply does not have an effect on choice blindness. Although we cannot exclude this explanation, it seems unlikely, especially for choices on the high and extreme end of the selfrelevance scale (i.e., such choices that one would describe as highly self-relevant). This leads directly to a second explanation of the current results: self-relevance may have an effect on choice blindness, but only for highly self-relevant decisions. Following this argument, the current results would indicate that we did not present our participants with decisions that were self-relevant enough to exhibit the effect. More specifically, it may not be sufficient to only ensure that the high and low self-relevance conditions differ in self-relevance, but self-relevance may also have to reach a high level in order to impact choice blindness rates. This may also explain the tentative support for the self-relevance hypothesis in the child sample. Although we have no objective evidence for this claim, it is possible that the choices made by the children were more relevant to them than those performed by adolescents. Indeed, the experimenters in Experiment 2 reported that the children were quite excited about the task and the choices they had to make. Such excitement was absent in the adolescent sample. Another possibility is that excitement or interest in the task has an effect on choice blindness.

A third explanation that is worth considering is that another factor interacts with self-relevance. While other factors are conceivable, we would like to focus on ambiguity as such a moderator. Merckelbach et al. (2011a) argued that evaluative decisions like those made in the choice blindness paradigm hold a certain level of ambiguity, making us prone to choice blindness. Accordingly, we may be unsure about an evaluative decision made at an earlier point (Sagana et al., 2014b). The role of ambiguity in choice blindness has been demonstrated in several studies that showed that blindness occurs not only for choices that are evaluative in nature but also for decisions that rely on long-term episodic memory (Sauerland, Schell, et al., 2013). Although speculative, it is possible that a self-relevance advantage for detections of secretly manipulated choices only occurs in situations of increased ambiguity. That is, self-relevance may be beneficial when ambiguity is high, but not when ambiguity is low. Following this argument, the current results would indicate that situational ambiguity was too weak in the current experiments for the effect to be revealed. This notion could be tested in future research.

For pure exploratory reasons, we also tested the tendency to act according to social demands as a possible mechanism underlying the choice blindness effect. Consistent with previous studies (Chapter 2; Sauerland, Sagana, et al., 2013; Sauerland, Schell, 
et al., 2013, Experiment 1 and 2), this idea was not supported by our data. Thus, the current data and the literature to date suggest that social desirability is not a crucial moderator of choice blindness.

To conclude, this work confirms the robustness of the choice blindness that is described in the literature across different materials and settings and demonstrates the validity of the effect across the life span. Furthermore, the effect has again shown to be largely unimpaired by different testing conditions. Future research should implement the choice blindness paradigm in settings that are likely to reveal limiting conditions of the effect. Choice blindness remains a fascinating effect which slips in our decisions, both the less, but also the more consequential ones; the one about what to wear, but also which doctor to visit. 



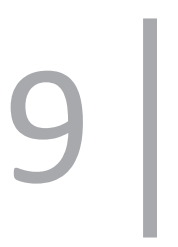

Memory impairment is not sufficient for choice blindness to occur 


\section{ABSTRACT}

Choice blindness refers to the effect that people can be easily misled about the choices they made in the recent past. The aim of this study was to explore the cognitive mechanisms underlying choice blindness. Specifically, we tested whether memory impairment may account for choice blindness. A total of $N$ $=88$ participants provided sympathy ratings on 10-point scales for 20 female faces. Subsequently, participants motivated some of their ratings. However, on three trials, they were presented with sympathy ratings that deviated from their original ratings by three full scale points. On nearly $41 \%$ of the trials, participants failed to detect (i.e., were blind) the manipulation. After a short interval, participants were informed that some trials had been manipulated and were asked to recall their original ratings. Participants adopted the manipulated outcome in only $3 \%$ of the trials. Furthermore, the extent to which the original ratings were accurately remembered was not higher for detected as compared with non-detected trials. From a theoretical point of view our findings indicate that memory impairment does not fully account for blindness phenomena. 


\section{INTRODUCTION}

Lay people often think that humans are permanently aware of the reasons that guide their decisions. In fact, the ability to compare the outcome of our choices with our intentions is vital for adaptive behaviour (Ridderinkhof, Ullsperger, Crone, \& Nieuwenhuis, 2004; Ullsperger \& von Cramon, 2004). Convinced of the rationality of their actions, people have strong opinions about their preferences and decisions. However, recent findings indicate that people are poor at detecting deviations from their choices. Specifically, according to choice blindness people often fail to detect a mismatch between their intention and the later outcome when their choice is surreptitiously manipulated. In the original demonstration of choice blindness (Johansson et al., 2005), participants had to select the more attractive alternative out of 15 different pairs of female faces. Subsequently, they were presented with the chosen alternative and were asked to explain their judgement. However, for three of these pairs the researchers, using a magic card trick, swapped the chosen photo with the alternative non-preferred face. Thus, participants had to justify a decision they never made. Astonishingly, only $13 \%$ of the manipulated trials were detected immediately at the time of the manipulation.

The basic effect of choice blindness has been widely replicated (e.g., Hall et al., 2010; Johansson et al., 2008; Chapter 2; Sauerland, Sagana, et al., 2013) in different domains that are highly relevant to everyday decision making, such as consumer preferences (Hall et al., 2010), moral decision making (Hall et al., 2012), and symptom reports (e.g., Merckelbach et al., 2011b). Choice blindness manipulations have been shown to result in changes of inclination towards a specific product and moral attitude, and in symptom escalation.

Yet, little is known about the mechanism underlying choice blindness. Previous studies have successfully ruled out a number of possible explanations, including poor encoding of the original (Johansson et al., 2005) or manipulated stimuli (Chapter 2; Sauerland, Sagana, et al., 2013), compliance (Johansson et al., 2008; Johansson et al., 2005; Chapter 2; Sauerland, Sagana, et al., 2013), suggestibility or the tendency to react in socially desirable ways (Merckelbach et al., 2011b; Chapter 2; Sauerland, Sagana, et al., 2013; Sauerland, Schell, et al., 2013). However, none of these studies specifically aimed at tackling the cognitive mechanisms underlying the effect. The present work is a first, but crucial step to fill in this gap.

Memory distortion seems intuitively the most plausible candidate to explain choice blindness. Memory distortion is closely related to the misinformation effect (Loftus \& Hoffman, 1989) and hindsight bias (Fischhoff, 1975) and in these domains, it possesses empirical merits. Interestingly, methods employed to study the misinformation effect and hindsight bias resemble the choice blindness paradigm. Specifically, all 
three involve exposure to an original stimulus, the subsequent confrontation with follow-up information (that may be misleading), and a final instruction that elicits the phenomenon ("Was there a stop sign?" "For which president candidate did you vote?" or "Why did you find this face the most attractive?"). Thus, all three phenomena can be conceptualized as forms of memory distortion caused by new information that influences a memory trace created earlier. Hence, it is reasonable to anticipate that the mechanisms underlying these phenomena share certain commonalities.

To explain misinformation and hindsight bias, researchers introduced the memory impairment framework (Fischhoff, 1975; Loftus \& Hoffman, 1989). According to this framework, the original memory trace is permanently distorted by the presentation of new information by means of alteration, erasure or a decrease in accessibility of the original memory trace. Accordingly, exposure to follow-up information can interfere with the recollection of the original trace, hence resulting in memory impairment. Extreme positions within this framework hold that the new information blends with the existing trace, resulting in an imminent and persistent alteration of the original trace (immediate assimilation hypothesis; Fischhoff, 1975). More conventional approaches postulate that the retrieval of separate memory traces depends on the recency and depth of the encoding (dual memory traces theory; Hell, Gigerenzer, Gauggel, Mall, \& Müller, 1988) or available congruent information (selective retrieval hypothesis; Morton, Hammersley, \& Bekerian, 1985; Slovic \& Fischhoff, 1977). Nonetheless, all accounts have in common that they view the interference of the new information as a cause of people's inability to recall the original trace (Mazzoni \& Vannucci, 2007). Thus, memory deterioration results from the failure to access the original memory trace.

Although memory deterioration is an intuitively plausible candidate to explain choice blindness, to our knowledge, there are no studies to date that have explicitly tested the memory-choice blindness link. Indirect evidence for such link comes from a field study (Chapter 4; Sagana et al., 2013) that examined choice blindness for eyewitnesses' facial recognition decisions, Sagana and colleagues (Chapter 4; 2013) reported that participants who made an accurate lineup decision were more likely to notice a covertly performed manipulation at the end of the experiment (i.e., retrospectively) than participants who made an erroneous recognition decision. In other words, participants' memory, as indicated by recognition accuracy, was associated with higher detection rates. Although this study did not provide a direct test of memory strength, these findings are broadly consistent with the idea that memory deterioration is responsible for blindness. On the other hand, participants who immediately noticed the change (i.e., concurrently) were not more accurate in their lineup decisions than participants who were blind to the change. This suggests that the accuracy of a recognition decision and the capacity to detect a manipulation are 
not always associated. Thus, Sagana et al. (Chapter 4; 2013) concluded that blindness for recognition decisions cannot be fully attributed to memory decay. The observation that blindness can be obtained even when the manipulated outcome is presented minutes after the original choice (Hall et al., 2012; Johansson et al., 2005) supports this position. Moreover, choice blindness may occur for stimuli of personal and moral significance (Hall et al., 2013; Sauerland, Schell, et al., 2013), again suggesting that memory decay may not be the sole mechanism driving the effect.

A direct test of whether memory impairment is a prerequisite for in choice blindness has not been done to date. This is the aim of the current study. To this end, we asked participants to rate the sympathy of female faces and secretly manipulated some of the judgments by increasing or decreasing their ratings. Shortly hereafter, participants were informed that some trials had been manipulated and they were asked to recall $50 \%$ of the original sympathy ratings they had made earlier. Given the common association between confidence and accuracy across different domains (Brewer \& Wells, 2006; Rolls, Grabenhorst, \& Deco, 2010; Yeung \& Summerfield, 2012), we also asked participants to provide a confidence rating.

Our research approach allowed us not only to measure memory strength, but also whether participants could sufficiently disentangle the original choice from the manipulated outcome once they had been notified about the manipulations. However, as we used a relatively short interval between the original ratings and the instruction to recall, one could argue that this is a limitation of the current a study. Specifically, one might argue that a relatively short interval may be suboptimal for studying memory corruption. However, given that participants were asked to recall ten different sympathy ratings of unfamiliar faces, we do think that we were able to tap into memory processes. Conversely, if a longer interval would have been introduced, our results would run the risk of being burdened by a disproportionate increase in cognitive load (Klimesch et al., 1993). Importantly, misleading post-event suggestions can impair memory with short retention intervals (Belli, Lindsay, Gales, \& McCarthy, 1994). A second feature of our approach is that, on the second round of trials, we asked participants to recall their evaluations of the faces. Asking participants to recall their original ratings may be problematic. Performing the task a second time, participants may simply re-evaluate the faces instead of recalling their original rating. Hence, consistency with the original choice may not reflect genuine recall, but a consistency in preference. This is a valid concern, as we have no means of knowing whether participants, indeed, recalled or re-evaluated. However, recent findings indicate that choice blindness manipulations can shape preferences in favour of the manipulated outcome in a second round of choices (i.e., reevaluation; Johansson et al., 2008; Johansson et al., 2013; Sauerland, Schell, et al., 2013). Importantly, such a shift was evident for both blind participants and detectors. Hence, we can make 
predictions about the outcomes for the reported ratings for cases where participants a) recalled or re-evaluated, b) memory is or is not impaired and c) participants are blind or detectors (see Table 9.1). For example, if our participants simply re-evaluated the faces, a shift in their answers in favour of the manipulated outcome should be expected, regardless of memory impairment being a valid explanation for choice blindness and participants being blind or detectors. If, however, participants recall as they were instructed - then blind participants and detectors should either differ in the reported ratings (i.e., impaired memory) or should both favour the original rating (i.e., non-impaired memory).

Table 9.1 | Hypothetical outcomes for reported ratings given the cognitive process, the memory capacity and participants' manipulation status.

\begin{tabular}{llll}
\hline Process & Memory & Manipulation Status & Reported Rating \\
\hline \multirow{2}{*}{ Recall } & Impaired & Blind & Manipulated \\
\cline { 2 - 3 } & Not-impaired & Detectors & Original \\
\cline { 2 - 4 } & Impaired & Detectors & Original \\
\hline \multirow{2}{*}{ Re-evaluation } & Blind & Original \\
\cline { 2 - 3 } & Not-impaired & Detectors & Manipulated \\
\cline { 2 - 3 } & Blind & Manipulated \\
\cline { 2 - 3 } & Detectors & Manipulated \\
\hline
\end{tabular}

To sum up, according to the memory impairment framework, the presentation of the manipulated outcome should hinder the recollection of the original rating. This view implies that participants will recall the original rating in the subsequent memory test more accurately for the non-manipulated compared with the manipulated trials. Additionally, participants aware of the change (i.e., detectors) are predicted to be able to discriminate between the two competing traces (original vs. manipulated) and thus be better able to recall the original rating than participants blind to the change. Furthermore, if the memory impairment framework is correct, blind participants will adopt the manipulated sympathy ratings as their own. Finally, detectors are predicted to display higher confidence in their ability to remember the original rating than blind participants. 


\section{METHOD}

\section{Participants}

A total of $N=88$ participants ( 27 men, $M_{\text {age }}=22.3$ years, $S D_{\text {age }}=5.0$, age range: $18-55$ ) took part in the study. Most of them were undergraduate psychology students $(90.9 \%)$, whereas the remaining participants $(9.1 \%)$ had various professional backgrounds. Student participants received course credit in return for their participation, while for the non-academics no monetary or other incentives were granted. Participation was voluntary. All participants were naïve to the purpose of the study and were tested individually. The study was approved by the standing ethical committee of the faculty.

\section{Stimuli and Stimulus Selection}

Twenty female facial photos were selected for the sympathy rating task. All faces were of British or Australian public features unknown to our participants and were extracted from a data base kindly provided by R. Jenkins (for full description of technical characteristics see, Jenkins et al., 2011). Photos showed the faces in roughly frontal aspect with neutral or smiling facial expression. Copyright restrictions prevent us from reproducing the images here. The size of the photos as presented on the computer screen was $4.5 \times 6.5 \mathrm{~cm}$ and they were centred in the upper half of the screen.

To avoid our results being attributed to differences in distinctiveness of the manipulated photos, as the effect of this factor on choice blindness is not yet examined, we selected three photos to serve as the to-be-manipulated targets, from the aforementioned stimulus pool. These had been rated the least distinctive, the most distinctive and moderately distinctive in a pilot study with 18 participants ( $11 \mathrm{men}, M_{\text {age }}=25.6$, $S D_{\text {age }}=8.31$, age range: $\left.19-48\right)$. These photos depicted Cilla Black $(M=3.9, S D=2.4)$, Rachel Stevens $(M=4.9, S D=1.7)$, and Carol Smilie $(M=5.9, S D=1.7)$. Distinctiveness scores for their pictures differ from each other, all $t s(17) \geq 2.18, p s \leq .058$. However, detection did not vary as a function of distinctiveness, Wald $\chi^{2} s(1, N=264)=1.98$, ps $\geq .376$. We will therefore not discuss this factor any further.

\section{Design}

The dependent variables were consistency with the original rating and consistency with the manipulated outcome. Detection (blindness vs. detection) of the manipulated trials and confidence in the ability to recall the original sympathy ratings served as the independent variables. As a measure of detection we used participants immediate (i.e., concurrent) apprehension of a change in their sympathy ratings. 


\section{Procedure}

All stimuli were presented on a computer screen at a resolution of $1024 \times 768$ pixels using Open Sesame display software (Mathôt, Schreij, \& Theeuwes, 2012). A cover story led participants to believe that the study was concerned with facial characteristics that make faces more or less sympathetic. The study consisted of two parts executed in a single session. During the first part, after signing the informed consent, participants were instructed to rate how sympathetic they found each of 20 female faces, using a scale ranging from 1 (not sympathetic at all) to 10 (extremely sympathetic). After a 2 seconds occlusion time, participants were presented with the picture and the corresponding sympathy rating again. Participants were asked to write down briefly the reasons for their ratings for half of the presented faces (i.e., 10). For the remaining faces, participants simply had to press the space bar to continue. However, in three of the 10 trials where a justification was required, we increased or decreased participants' original sympathy ratings by three full points. For each of the three trials, the manipulation was dependent on participants' sympathy rating. Specifically, if participants provided a rating from 1 and 5, we increased their rating, but if participants provided a rating from 6-10, we decreased their initial rating. Detection was not affected by the direction of the manipulation, all $x^{2}(1, N=264)=2.16, p=.143$, phi $=-.09$. If participants typed a comment indicating that the displayed rating did not correspond with their own or that the program had made a mistake, they were classified as detectors. Detectors frequently also verbally informed the experimenter about the change i.e., the "mistake".

Following the completion of the sympathy rating task participants filled out personality questionnaires as a filler task. Subsequently, the second part of the study began, in which participants were informed that some of their sympathy judgments had been altered. No information was given as to which specific trials or how many of them had been manipulated. Participants' task was to again view the 10 faces for which they had provided a motivation earlier and to recall their original sympathy ratings. Furthermore, participants indicated how confident they were that this corresponded with their original rating on an 11-point scale ranging from $0 \%$ to $100 \%$. Subsequently, as in previous studies (e.g., Hall et al., 2010; Johansson et al., 2005; Chapter 2; Sauerland, Sagana, et al., 2013; Sauerland, Schell, et al., 2013), participants received a questionnaire to determine whether they had detected the manipulations before we disclosed that information, but did not report it while performing the task. Given that many participants were confused by the structure of the questionnaire ${ }^{5}$, it is doubtful

$5 \quad$ Participants were asked whether they had detected the manipulation and if so, they had to specify how many times the manipulation had occurred and which trials exactly had been manipulated. Unfortunately, we did not include the actual photos in the post-test questionnaire, an omission that caused confusion in the participants about which trial referred to which picture. However, only 
whether the trials reported in the Post-test questionnaire reflect genuine detections. Therefore, we will not further address the questionnaire data. Finally, participants were thanked and fully debriefed.

\section{RESULTS}

\section{Choice Blindness}

Out of the $88 \times 3=264$ manipulated trials, 107 (40.5\%) were detected. Table 9.2 shows the detection rates for the three manipulated trials and across all 264 manipulated trials. One could argue that strictly speaking, choice reversal only occurs when manipulations involve changes crossing the mid-point of the rating scale (i.e., from "sympathetic" to "not sympathetic", rather than from "sympathetic" to "less sympathetic"). Accordingly, we reran the analysis including only those participants, for which the manipulation resulted in the original choice being shifted to the opposite half of the Likert scale, leaving 221 trials for analysis. Ninety-one (41.6\%) of these were detected and the pattern results for all subsequent analyses remained largely the same. Therefore, we report the analyses for the total sample.

Table 9.2 | | Proportion detection for the three manipulated trials.

\begin{tabular}{lcl}
\hline & \multicolumn{2}{c}{ Detection } \\
\cline { 2 - 3 } & $M(\%)$ & $95 \% \mathrm{Cl}$ \\
\hline $1^{\text {st }}$ manipulation & 35.2 & $25.0-45.0$ \\
\hline $2^{\text {nd }}$ manipulation & 39.8 & $29.5-50.0$ \\
\hline $3^{\text {rd }}$ manipulation & 46.6 & $43.2-64.7$ \\
\hline Overall & 40.5 & $38.8-47.0$ \\
\hline
\end{tabular}

\section{Memory Impairment}

To determine whether participants' ability to remember the original ratings was impaired by the performed manipulations, we first compared memory for the sympathy ratings in manipulated vs. non-manipulated trials. For the non-manipulated trials, participants were able to exactly remember their original rating in $45.8 \%$ (278 of $607,95 \% \mathrm{Cl}[42.0,49.0])$ of the trials. For the manipulated trials, that percentage was $46.2 \%$ (122 of $264,95 \% \mathrm{Cl}[40.2,52.3])$. Evidently, there were no differences in participants ability to remember their original rating, $z=-0.91, p=0.91$, indicating

an additional $19(7.2 \%)$ of the 264 trials were accurately reported in the Post-test questionnaire raising the total detection rate to $47.7 \%$ and the pattern of results remained largely the same as for concurrent detection. 
that the presentation of the manipulated item does not impair the memory for the original choice.

Next, we examined whether participants would adopt the manipulated sympathy ratings as their own as a result of impaired memory of the original choice. Comparisons of participants' consistency with the manipulated outcome vs. consistency with the original rating revealed that participants were much more often consistent with their original rating than with the manipulated one, McNemar $\chi^{2} s(1, N=264)=$ $101.73, p<.001$. Specifically, across the 246 manipulated trials, participants reported the manipulated outcome in $2.7 \%$ of the trials $(S D=16.0,95 \% \mathrm{Cl}[0.8,4.9])$, while participants were consistent with their original rating in $46.6 \%(S D=49.9,95 \% \mathrm{Cl}[40.2$, 52.7]) of the trials. In the remaining trials $(50.8 \%, S D=50.1,95 \% \mathrm{Cl}[44.3,56.8])$, participants were inconsistent with both the manipulated outcome and the original rating (see Figure 9.1). Applying a more liberal consistency criterion, of \pm 1 scale point ${ }^{6}$ as equivalent to the original rating or the manipulated outcome led to similar results, McNemar $\chi^{2}(1, N=264)=156.49$, ps <.001. Specifically, 9.5\% (SD = 29.3, 95\% Cl [6.1, 13.3]) of the 246 manipulated trials were similar to the manipulated outcome, while $84.5 \%$ (SD $=36.3,95 \% \mathrm{Cl}[79.6,88.6])$ were consistent with the original rating, and $6.1 \%(S D=23.9,95 \% \mathrm{Cl}[3.4,9.1])$ with neither of the trials. Apparently, manipulating the original sympathy rating did not lead to overall memory distortion.

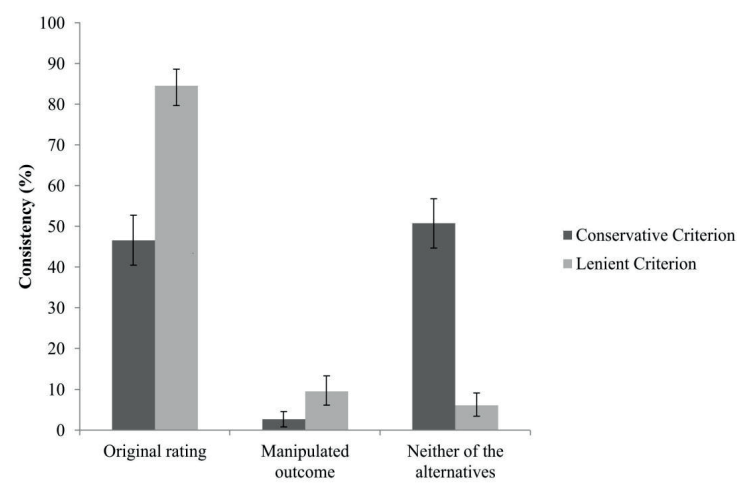

Figure 9.1 | Proportion of trials consistent with the original sympathy rating, the manipulated outcome, and neither of both after the application of a conservative or lenient ( \pm 1 scale point variance) criterion. Error bars represent $95 \%$ confidence intervals.

6 This is equal to roughly one SD difference in consistency with the original rating $(S D=0.89)$ and the manipulated choice $(S D=1.07)$. 
Next, we tested the hypothesis that consistency with the original rating varies as a function of detection. No support was found for this assumption, $x^{2}(2, N=264)=2.38$, $p=.122$, phi $=.09$. Specifically, consistency with the original sympathy rating was met in $42.7 \%(S D=49.6,95 \% \mathrm{Cl}[35.1,50.7])$ of the non-detected and in $52.3 \%$ ( $S D=50.2$, $95 \% \mathrm{Cl}[42.9,61.7])$ of the detected trials. Thus, memory impairment alone is not a sufficient explanation for choice blindness.

In light of these findings, we wondered whether our manipulations had any effect on participants recall ability. To test this, we computed the deviation between the remembered and participants' original sympathy ratings (i.e., Consistency = rating time 2 - rating time 1 ) as a continuous measure of consistency with the original rating. Our aim was to examine whether this alternative approach would reveal differences in recall ability between manipulated and non-manipulated trials. Two outliers with a deviation of five scale points from the original choice were excluded. A paired sample $t$-test showed that the mean distance between the recalled and the original sympathy rating was greater for manipulated $(M=.53, S D=.49,95 \% \mathrm{Cl}[.43, .64])$ than nonmanipulated trials $(M=.27, S D=.22,95 \% \mathrm{Cl}[.22, .31]), t(85)=4.59, p<.001$. Additionally, the direction of the change from participants' original to their remembered estimate was consistent with the direction of the manipulation (although it did not affect the distance between participants' original and remembered estimate, $t(260)$ $=1.75, p=.080$ ). Figure 9.2 displays the mean distance from the original sympathy ratings for increase $(n=123)$ and decrease $(n=141)$ manipulations. As can be seen, the manipulations affected participants' recollections.

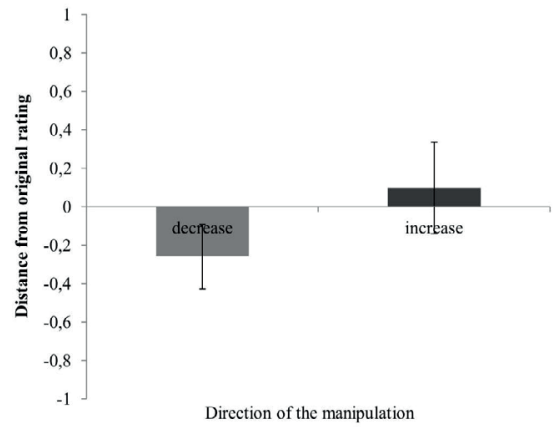

Figure 9.2 | Mean distance from the original sympathy rating as a function of manipulation direction (increase vs. decrease). Error bars represent $95 \%$ confidence intervals.

Following a similar approach, we next examined whether this continuous measure of consistency would reveal differences in recall ability between detected and 
non-detected trials. Hence, we performed Generalized Estimating Equations (GEE) analyses with consistency with the original sympathy estimate as dependent variable and detection (blindness vs. detection) as predictor. However, no significant results emerged, Wald $\chi^{2}(1, N=262)=2.7, p=.141$. Figure 9.3 displays the mean distance from the original sympathy ratings as a function of detection. Finally, we tested whether the distance from the original sympathy rating would be greater for the non-detected compared with the detected trials among the inconsistent with the original rating cases $(N=139)$. No support was found for this assumption, Wald $\chi^{2}(1$, $N=139)=0.20, p=.652$. Specifically, the mean distance from the original sympathy rating for the non-detected trials was $M=1.45$ ( $S D=.76,95 \% \mathrm{Cl}[1.30,1.63])$ and for the detected trials was $M=1.39(S D=.85,95 \% \mathrm{Cl}[1.18,1.63])$. Evidently, memory impairment is not sufficient for choice blindness to occur.

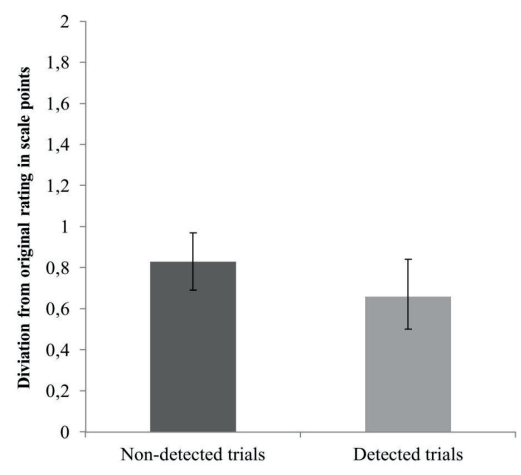

Figure 9.3 | Mean distance from the original sympathy rating as a function of detection (detected vs. non-detected). Error bars represent 95\% confidence intervals.

\section{Confidence as Postdictor of Consistency}

The final set of analyses tested whether confidence in recall ability, along with detection, can postdict consistency with the original sympathy rating. We performed GEE analyses with consistency with the original sympathy estimate as dependent variable and detection (blindness vs. detection) and confidence as predictors. In 14 trials, no confidence rating was provided leaving 250 trials for analysis. The interaction effect between detection and confidence was not significant, Wald $\chi^{2}(1, N=250)=2.80, p=$ .094. The model without the interaction term returned no significant effect for detection, Wald $\chi^{2}(1, N=250)=.621, p=.431$, but revealed a significant confidence effect. After deleting detection from the model, confidence attained significance, Wald $\chi^{2}(1$, $N=250)=9.33, p=.002$. Specifically, higher confidence ratings were associated with higher consistency with the original choice. Thus, confidence seemed to be a reliable 
predictor of consistency with the original rating. Figure 9.4 presents the confidence ratings for all manipulated trials that are consistent or inconsistent with the original rating.

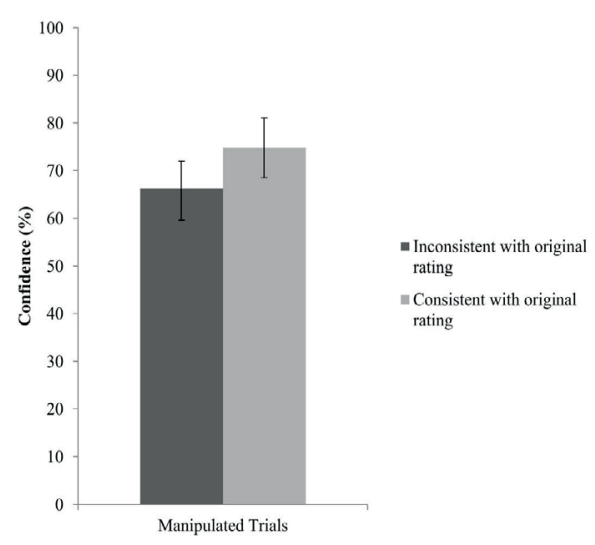

Figure 9.4 Confidence as a function of consistency with the original sympathy rating for all manipulated trials. Error bars represent $95 \%$ confidence intervals.

Using the continuous measure of consistency led to comparable results. Specifically, neither the interaction between detection and confidence, Wald $\chi^{2}(1, N=249)=0.93$, $p=.334$, nor the main effect of detection (after deletion of the interaction effect) was significant, Wald $\chi^{2}(1, N=250)=.602, p=.438$. The main effect of confidence indicated that high confidence was associated with little deviation from the original rating, while lower confidence was associated with stronger deviations from the original rating, Wald $\chi^{2}(1, N=249)=5.96, p=.015$. Figure 9.5 displays mean confidence across the observed distances from the original rating.

\section{DISCUSSION}

The aim of the current study was to test whether choice blindness is a result of memory impairment of the original trace. To our knowledge, this is the first study that directly examined this issue. Drawing on the memory impairment framework (Fischhoff, 1975; Loftus \& Hoffman, 1989), we predicted that participants would have difficulties in remembering their original sympathy rating after confrontation with a manipulated rating and that they would adopt the manipulated outcome as their own. Contrary to this assumption, the vast majority of the participants gave sympathy ratings that were consistent with their original choice. Further, there were no differences between de- 


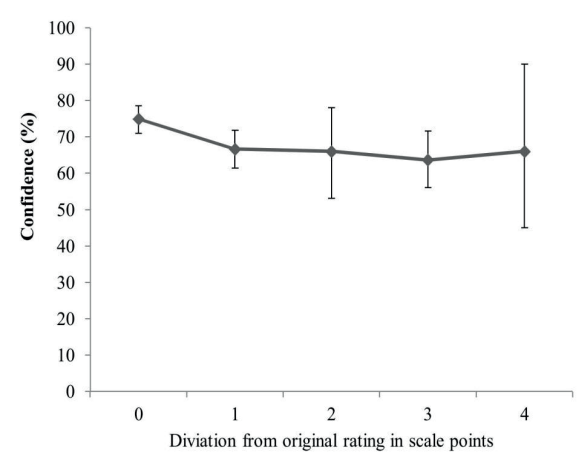

Figure 9.5 | Confidence as a function of the deviation from the original sympathy rating across all manipulated trials. Error bars represent $95 \%$ confidence intervals.

tectors and blind participants in their ability to reproduce their original ratings. This was true when consistency with the original ratings was expressed in dichotomous terms, but also while being indexed in a continuous fashion. Although participants were affected by the direction of the manipulation, the distance between the recalled and the original sympathy rating did not differ among detected and non-detected trials. Furthermore, participants' confidence in their ability to remember the original sympathy rating was a reliable postdictor of consistency.

Despite the fact that choice blindness can occur in the absence of memory impairment, the current study allows for other important observations. First, our results provide further evidence for the robustness of the choice blindness effect (e.g., Hall et al., 2010; Johansson et al., 2008; Chapter 2; Sauerland, Sagana, et al., 2013). Second, our findings are comparable with the broader misinformation literature which shows that post-hoc misinformation often distorts memory (for a review see Ayers \& Reder, 1998; Loftus \& Hoffman, 1989; Payne, Toglia, \& Anastasi, 1994). Evidently, there were differences in the remembered sympathy rating between manipulated and non-manipulated trials and participants were largely affected by the direction of the manipulation (see Figure 9.2). The fact that consistency with the original sympathy rating was not associated with the capacity to detect a manipulation though, speaks to the idea that distorted memory is not necessary for choice blindness to occur.

Third, the current study is informative as it demonstrates with a direct test that blindness cannot be attributed to impaired memory of the initial memory trace or memory decay. Specifically, in a substantial number of trials (123 of 264; 46.6\%), participants were able to remember their original rating with precision. This supports the idea that the memory trace for the original rating was still accessible and could be retrieved at will. These findings are in line with earlier indications that blindness 
cannot be reduced to weak memory or forgetting (Chapter 4; Sagana et al., 2013). Additionally, in those cases that participants changed their ratings from the original sympathy ratings to the subsequently remembered sympathy estimates $153.4 \%$ of trials), the shift in the answers was independent of whether a manipulation was detected or not. Thus, even when participants were aware of the change in their judgments, they had limited capacity to segregate the influence of the manipulation from their recollections. This replicates earlier findings showing that participants shift their answers in favour of the originally non-preferred alternative when asked to perform a second round of choices (Hall et al., 2013; Johansson et al., 2008; Sauerland, Schell, et al., 2013). These studies and our findings are consistent with work demonstrating that there are conditions in which choices precede preferences (rather than vice versa; see studies by Sharot, Martino, \& Dolan, 2009; Sharot, Velasquez, \& Dolan, 2010).

Further, our results demonstrate that confidence is a reliable postdictor of consistency with the original sympathy rating. Hence, the present findings indicate the informative value of confidence as a postdictor of the accuracy of estimates provided following a manipulated outcome; that is, following incorrect information about one's own decision. Considering that to date we have only limited understanding of the mechanism contributing to choice blindness - and thus are incapable of offering methods for its reversal - the postdictive value of confidence might be beneficial for establishing the accuracy of a remembered choice.

Turning to the limitations of the present study, as already discussed in the introduction, participants may not have engaged actively in retrieval, but rather may have provided a sympathy estimate as if they were asked to perform the same task a second time. However, the fact that both blind participants and detectors were consistent with their original rating speaks to the idea that participants, indeed, recalled their original rating instead of simply performing the task a second time (see Table 9.1). Furthermore, our procedure was largely parallel with what is typically done to evaluate memory for past decisions in hindsight bias literature (e.g., Hoffrage, Hertwig, \& Gigerenzer, 2000; Pohl, 2007; Sanna \& Schwarz, 2003). Nevertheless, the contributions of retrieval versus preferential consistency should be addressed more directly in future research. Another limitation is that the post-test questionnaire was handed out at the end of the experiment, after participants were informed about the manipulations. This made it difficult to derive retrospective detection rates. Additionally, the absence of the actual stimulus photos in the post-test questionnaire was a source of confusion. Future research should consider administering the post-test questionnaire before the recall task and including photos of the stimuli instead of verbal descriptions.

To summarize, this study has shown that memory impairment is not sufficient prerequisite for choice blindness to occur. Instead, our findings indicate a complex 
mechanism responsible for choice blindness that allows a fairly accurate recollection of the original choice but yet disables the detection of a manipulation. This arguably hints in the direction of a subtle malfunction in recognition. One way to test this interpretation is to examine differences in neuronal activity while participants perform a typical choice blindness paradigm and a subsequent recognition task. We acknowledge though that developing a choice blindness paradigm which permits the collection of neural data may be difficult, given that $\mathrm{FMRI}$ and ERP measures require a number of repetitive trials which in itself may introduce artefacts. An alternative explanation is that participants are involved in a self-persuasion process (Johansson et al., 2011). Endorsing choices suggested by others may generate a degree of cognitive dissonance, which people want to overcome (Festinger, 1962; Henkel \& Mather, 2007). Therefore, participants confabulate introspective arguments to convince themselves about the assumed choice. Hence, they fail to detect the manipulated outcome but are able to recall their original choice when the dissonance is resolved by the revelation that the choice had been manipulated.

In conclusion, we believe that the results provide interesting insights for understanding choice blindness, with the major finding being that forgetting is not an exhaustive explanation for the phenomena. Hence, to grasp the fundamentals of choice blindness, we may better consider Alan Baddeley's (1988) question: "What the hell is it for?". Therefore, we suggest that future research should focus on the function of blindness when answering the question about why they occur at all in everyday life. $s$ 


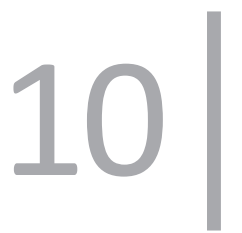

General Discussion 



\section{AIMS OF THE PRESENT DISSERTATION}

The studies described in the present dissertation aimed to 1) establish the relevance of choice blindness in eyewitness testimony, 2) explore the moderating factors of the effect of blindness in eyewitness testimony, and 3) unravel the mechanisms underlying choice blindness. In the introductory chapter, we bridged the gap between the witness testimony and the choice blindness literature on theoretical grounds. In Part 1 , we built upon these theoretical grounds and established the occurrence of blindness in ear and eyewitness recognition decisions. Through an in depth investigation, we associated blindness for evaluative and categorical decisions and established that witnesses can be susceptible to blindness manipulations. In Part 2, we extrapolated our findings to the field. In two studies, we replicated earlier work indicating that choice blindness occurs for eyewitness recognition decisions and extended our knowledge by showing that blindness occurs under conditions that come close to real life eyewitness setting. Furthermore, we demonstrated that blindness can occur for choosers and nonchoosers, as well as for target-present and target-absent lineups.

In Part 3, we expanded on the application of the choice blindness paradigm in other eyewitness settings. We first explored the impact of own-race bias on eyewitnesses' blindness for identification decisions. We observed that manipulations of other-race identifications were less likely to be detected compared with manipulations of ownrace identifications, providing tentative support for the premise that blindness rates could be moderated by ambiguity. Subsequently, we examined whether participants would be able to detect blindness manipulations in their written statements for a variety of retention intervals. We found that a substantial proportion of manipulations in eyewitnesses' written statements went undetected even within minutes after giving testimony.

In Part 4, we took a step away from the forensic field and examined potential moderators and explanations for choice blindness. We first focused on the role of self-relevance as a moderator, but found only weak support for the hypothesis that self-relevance can decrease blindness rates. Finally, we investigated memory impairment and forgetting as a potential explanation of choice blindness. We found little evidence for this idea. In the following, we discuss the main findings of the studies, their implications for the judicial system, as well as directions for future research. 


\section{SUMMARY AND DISCUSSION OF THE RESEARCH FINDINGS}

\section{Blindness in Eyewitness Testimony}

In a series of twelve experiments, as described in Parts 1-3 of the present dissertation, we have shown that choice blindness is relevant for ear and eyewitness testimony. Table 10.1 provides a summary of the concurrent and retrospective blindness rates as observed in the studies of this dissertation. Examining the effect of blindness manipulations on earwitnesses (Chapter 2), we found that choice blindness transfers to auditory stimuli, with the overwhelming majority of participants being blind to our manipulations (71-81\%; see Table 10.1). The observed blindness rates come close to those reported in choice blindness studies using facial stimuli (73-88\%; Johansson et al., 2008; Johansson et al., 2005) and are comparable to the choice blindness rates found for olfactory and gustatory stimuli (67-86\%; Hall et al., 2010). Thus, this study replicates and extends previous work thereby showing that choice blindness is a robust effect that seems to operate in a comparable way for different senses.

Following the successful application of blindness manipulations for auditory stimuli, in Chapter 3 we aimed to identify the conditions under which blindness phenomena occur in facial recognition decisions. We began our endeavour (Experiment 1) wondering whether absolute recognition decisions (i.e., correct or incorrect) would affect blindness rates to a similar extent as preference judgements, the outcome of which is evaluative in nature. Unlike absolute decisions, evaluative judgments about a person may change depending on mood or context (Loewenstein \& Small, 2007), making decisions more susceptible to blindness. Hence, we hypothesized that in an eyewitness setting, choice blindness rates would depend on decision type (evaluative vs. absolute). This hypothesis was only partially supported. Although concurrently there were no more blind participants for the evaluative compared with the absolute decisions, in retrospect absolute decisions yielded lower blindness rates. Interestingly, blindness for evaluative decisions in the eyewitness setting were decreased compared with studies where participants performed evaluative judgements of visual stimuli (Johansson et al., 2008; Johansson et al., 2005). This finding suggests that the eyewitness setting is to some extent different from the settings in which choice blindness has been studied in so far. That is particularly evident in Experiments 2a-c where, by means of a simple recognition procedure, we found moderate concurrent blindness rates and virtually no retrospective blindness in student and non-student samples. In explaining this discrepancy, one should consider the moderating factors of the effect. We turn to this point later in the discussion (see Moderators of Choice Blindness). Finally, to increase the ecological validity of our findings, in Experiment 3 of Chapter 3 we inserted a $48 \mathrm{hr}$ interval between the presentation of the recognition task and the presentation of the manipulated outcome. Under these conditions, blindness rates 


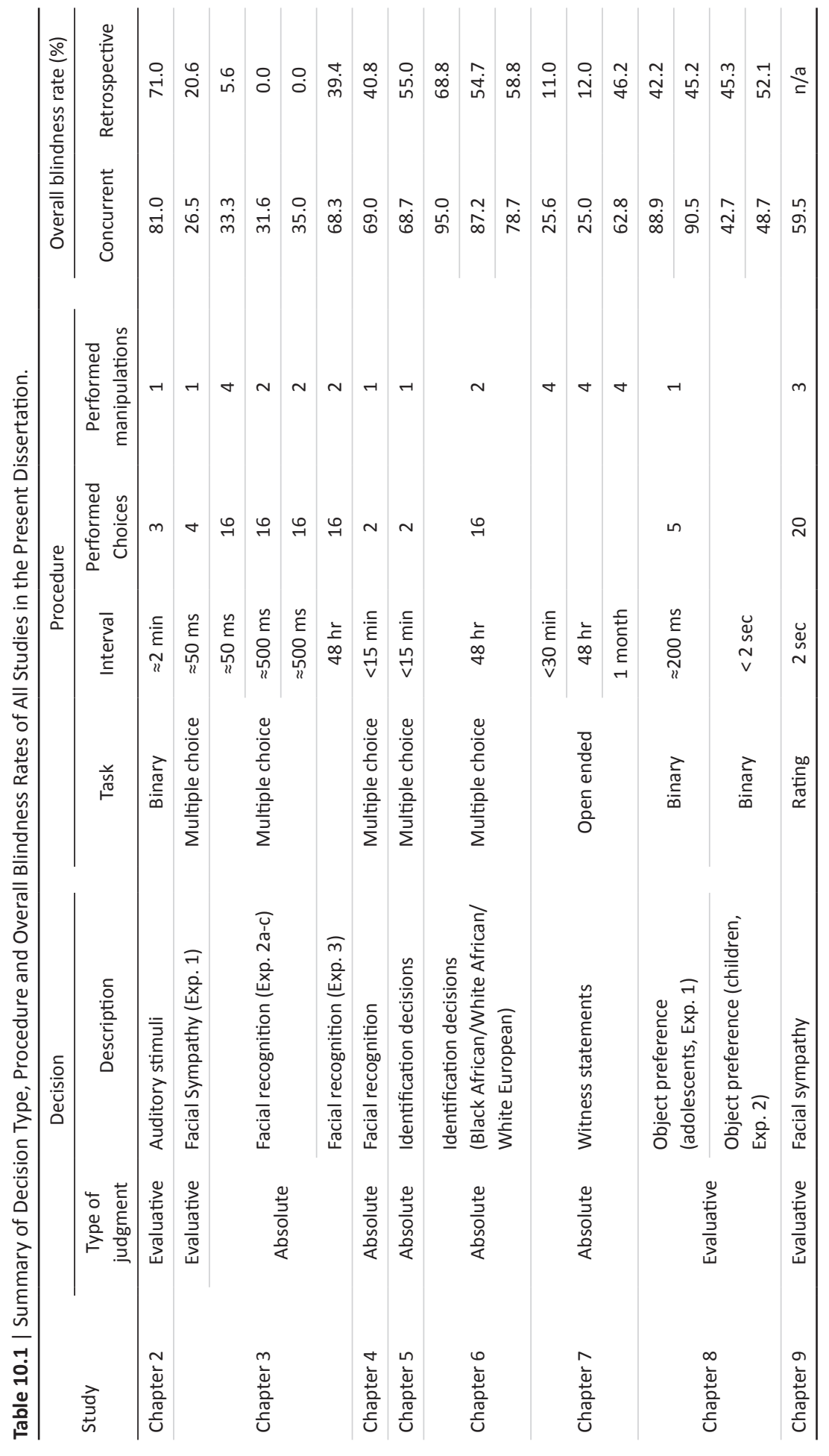


increased dramatically compared with the previous three recognition experiments (see Table 10.1). Therefore, we can conclude that blindness phenomena occur for eyewitness recognition decision and even more so when retention intervals come close to real life conditions.

Establishing the effect in the laboratory is certainly a first step in the right direction. Yet, a laboratory environment falls short in imitating real life conditions. Therefore, to confirm the occurrence of the effect under naturalistic encoding conditions and hence affirm its relevance for the judicial system, we conducted two additional experiments employing a field study methodology. Using staged non-criminal (Chapter 4) and criminal (Chapter 5) events, we replicated our laboratory findings and showed that blindness for identification decisions can occur at an alarmingly high level in real life settings (41-69\%). Over and above the replication of earlier findings, we extended our knowledge by demonstrating that blindness for identification decisions occurs for choosers and, to a lesser extent, nonchoosers, as well as for target-present and, to a lesser extent, target-absent lineups (Chapter 5). Bearing in mind our laboratory and field study findings, it is noteworthy that the magnitude of blindness for selections under naturalistic encoding conditions exhibits remarkable stability across studies (see Table 10.1). This once more signifies the robustness of the effect. Nonetheless, the lower blindness for nonchoosers than choosers speaks to the idea that choice blindness is limited by the rationality of human mind. Specifically, participants must have found it harder to justify a choice reversal than a choice exchange and therefore exhibited higher blindness rates for the latter.

In light of the stable nature of blindness for identification decisions, we aimed to investigate whether blindness could be relevant for other aspects of eyewitness testimony. In Chapter 6, we investigated the impact of the own-race bias on blindness for one's own eyewitness identification decisions. We hypothesized that making other-race identifications would increase blindness rates compared with own-race identifications due to the increased cognitive effort inherent in other-race identifications (Brigham \& Malpass, 1985; Chiroro \& Valentine, 1995). The results supported our assumption as participants who made other-race identifications were more likely to be blind to the manipulations (69-95\%) than participants who made own-race identifications (55-87\%; see Table 10.1). However, as the design of the study was not fully crossed, we should be careful before attributing the findings solely on the crossrace identifications and not to general responding differences between the groups or social influences. Nevertheless, these results clearly show that estimator variables can hinder the detection of a manipulation. Additionally, the results provided further support for the idea that blindness for identification decisions occurs for choosers and nonchoosers and for target-present as well as target-absent lineups. However, we did not replicate the detection advantage of nonchoosers over choosers which 
was evident in our field study. We speculate that the discrepancy could be attributed to the increased number of identification decisions (4 vs. 1) and the longer retention interval (48 hr vs. minutes) between the identification and the presentation of the manipulated outcome for the laboratory compared to the field study. Both these factors could have shaken participants' trust in the memory of rejecting a lineup, resulting in choice reversals being as easily accepted as choice exchanges. However, before we can draw firm conclusions about the effect of choice on blindness more conceptual replications are needed.

Subsequently, in a series of three studies, we examined the ability of eyewitnesses to detect manipulations introduced to their written statements (Chapter 7). Results revealed that a substantial proportion of manipulations in eyewitnesses' written statements went undetected even within minutes after giving testimony. Our findings are in line with earlier work showing that witnesses accept unreported details as their own testimony, even when they are requested to actively search for errors (Experiment 1; Christianson et al., 2007). Furthermore, our results are in agreement with recent findings indicating that blindness phenomena can occur in the context of people's autobiographical accounts (Sauerland et al., in press; Sauerland, Schell, et al., 2013). Importantly, these findings are consistent with our earlier work on blindness for identification decisions, showing that blindness manipulations can exert their influence in various aspects of the eyewitness testimony. Certainly, in the conventional way of thinking, reporting details of a witnessed event does not constitute a choice. Thus, it could be argued that manipulations in one's statement are unlike manipulations in identification decisions. Taking into account Koriat and Goldsmith's monitoring and control framework (Goldsmith et al., 2002; Koriat \& Goldsmith, 1996) however, each piece of information that is to be reported undergoes a decision making before it is volunteered as an answer. Thus, in this and in our point of view, an identification decision and the decision to report specific information share a striking resemblance.

In sum, through extensive experimentation, we have established that blindness for witness testimony is an effect of non-trivial magnitude. The robust nature of the effect is remarkable as the effect of choice blindness operates on different modalities and aspects of witness testimony, under laboratory and naturalistic conditions. Consequently, understanding choice blindness is important not only because of their immediate significance for the legal practice but also because they could add to our knowledge about the decision making errors of witnesses.

\section{Moderators of Choice Blindness}

Part 4 is partially devoted to tackling specific moderators of blindness in the witness setting. However, the pursuit and discovery of meaningful moderators is not restricted to the two chapters of Part 4. Rather, it expands across all sections of this dissertation. 
In the following, we provide an overview of all the limiting and facilitating factors of blindness encountered in our studies as we attempt to demonstrate that, with the possible exception of self-relevance, all these conditions can be conceptualized as different aspects of a single key moderator, namely ambiguity.

\section{Retention interval}

The retention interval between a choice and the presentation of the manipulated outcome seems to be a determining factor for the intensity of blindness. In Chapter 3 moving from shorter (milliseconds) to longer ( $48 \mathrm{hr}$ ) retention intervals practically doubled the blindness for recognition decisions (see Table 10.1). Similarly, in Chapter 7 longer retention intervals ( 1 month) were associated with rates of blindness for manipulations in one's written statement twice as high as those observed for shorter retention intervals (minutes and $48 \mathrm{hr}$ ). Similar findings have also been reported for manipulations of autobiographical memories (Sauerland, Schell, et al., 2013) with blindness rates increasing from $25 \%$ after 10 minutes to $45 \%$ after one week. The retention interval seems to exert its influence through compromising memory strength for the original choice. Yet, considering that participants can be blind to manipulations of choices performed seconds ago, for which memory should be strong, blindness should not be equated with weak memory (see "Commentary on the effect of accuracy on blindness in eyewitness testimony"). Hence, the retention interval between the original choice and the manipulated outcome seems to be merely a facilitating factor of blindness and not the primary mechanism.

\section{Stimulus similarity}

Another recurring determining factor of blindness seems to be the similarity between chosen versus manipulated stimuli. Specifically, in Chapter 2 we found that a high similarity between chosen and manipulated voice increased concurrent (but not retrospective) blindness rates. Likewise, in Chapter 4 we observed that high similarity between the originally chosen and the manipulated lineup member increased concurrent and retrospective blindness rates. Previous authors have also reported an effect of similarity for a limited selection of stimuli (Hall et al., 2010), although others have not found the effect (Johansson et al., 2005). In Chapter 2, however, the post-hoc voice ratings showed that the low-similarity voices also differed in distinctiveness, whereas the high similarity voices did not. This, points to the possibility that, in addition to low similarity, high distinctiveness may create a boundary condition for the effect. Earlier choice blindness studies which looked into the role of stimulus similarity have not addressed the issue of distinctiveness (Hall et al., 2010; Johansson et al., 2008; Johansson et al., 2006). Consequently, it is conceivable that the effects of similarity may align with distinctiveness. Nevertheless, our results in Chapter 4, where distinc- 
tiveness was controlled for, speak in favour of similarity as a boundary condition for blindness. This does not imply that distinctiveness cannot be an additional limiting condition. It rather suggests that stimulus similarity alone can moderate the effect.

\section{Self-relevance}

Another possible moderator of blindness is self-relevance. The effect of self-relevance denotes the tendency of people to encode information in which the self is implicated in a privileged way (Rogers et al., 1977) resulting in superior encoding and recognition for self-relevant that non self-relevant events (Symons \& Johnson, 1997). Therefore, in Chapter 8 we predicted that decisions high in self-relevance should lead to lower blindness rates than those low in self-relevance. We found only little evidence in support of this idea. For adolescents no effect of self-relevance on choice blindness was found. For children self-relevance moderated retrospective, but not concurrent detection for the toy manipulation. The finding is certainly counterintuitive and, although we cannot exclude the possibility that self-relevance is not a moderator of blindness, we ought to consider alternative explanations. One possible explanation is that self-relevance has an effect on blindness, but only for decisions that score high on the self-relevance scale, as for example when a person's dignity or freedom is at stake. A second explanation is that self-relevance interacts with ambiguity. Ambiguity refers to vagueness and imprecision in the stimuli that allow for multiple interpretations (Sloman et al., 2010). Therefore, it may be that self-relevance is beneficial when ambiguity is high. However, whether these interpretations are applicable remains to be empirically tested. Yet, considering the potential role of ambiguity in relation to self-relevance, we believe it is worth turn to ambiguity as a moderator of choice blindness.

\section{The key moderator: Ambiguity}

In the context of eyewitness testimony, the retention interval and stimulus similarity basically appear to increase the ambiguity of the task. Evidently, the longer the retention interval between the original choice and the presentation of the manipulated outcome, the harder it should be to detect a discrepancy between the choice and its outcome. Analogously, the higher the similarity of (or lower the discrimination between) the original choice and the manipulated outcome, the harder it should become to detect the manipulation. Therefore, ambiguity is a wider construct that, compared with retention interval and stimulus similarity, incorporates additional elements which are known to influence decision making including mood or context (Loewenstein \& Small, 2007).

Ambiguity was originally proposed as an explanation of blindness (see Merckelbach et al., 2011a). However, it soon became clear that although ambiguity is an essential 
moderator, it cannot explain how choice blindness arises. As a moderator, however, ambiguity seems to accommodate all of the observed effects. Specifically, retention interval, and stimulus similarity can all be conceptualized as factors that increase ambiguity in a choice blindness task. Importantly, ambiguity can also account for the reduced blindness rates for absolute versus evaluative tasks (Experiment 1, Chapter 3) because evaluative decisions are by definition more ambiguous than absolute ones since they can be affected by external factors such as the mood of the observer (e.g., older adults looking toward positive and away from negative faces when in a bad mood; Isaacowitz, Toner, Goren, \& Wilson, 2008) or the target (e.g., persons are less attractive when posing sad than neutral or happy expressions; Mueser, Grau, Sussman, \& Rosen, 1984). Finally, although this remains to be empirically tested, self-relevance may interact with the task ambiguity in such way that self-relevance is beneficial when ambiguity is high. Therefore, we believe that ambiguity is the key moderator of blindness. In support for this notion are findings indicating that people use ambiguity in a self-serving manner (Shalvi, Eldar, \& Bereby-Meyer, 2012; Shalvi, Gino, Barkan, \& Ayal, 2015). In ambiguous situations people may find it easier to come up with justification and confabulations on why and how the manipulated outcome must be the originally chosen one. In fact, ambiguity, self-justification and their role in principle violations is becoming a productive are of research, which underlined the importance of ambiguity in other settings than choice blindness.

\section{The Underpinnings of Choice Blindness}

Part 4 of the present dissertation is also devoted to understanding the mechanism underlying choice blindness. Before one can investigate the underlying mechanism, one should first thoroughly establish the effect. Since we only recently made the transition from establishing the effect to unravelling the mechanism, we so far only managed to investigate memory decay as a potential candidate. Drawing from the memory impairment framework (Fischhoff, 1975; Loftus \& Hoffman, 1989), in Chapter 10 we hypothesized that the presentation of the manipulated outcome should hinder the recollection of the memory trace of the original choice. Furthermore, we expected the impairment to be more pronounced for blind participants than for detectors. Contrary to our assumption, participants were able to recall their original choice with reasonable precision (recall accuracy: 46.2\%-85.4\%). Furthermore, no differences between detectors and blind participants emerged in their ability to reproduce their original choice. We concluded that impaired memory of the initial memory traces or memory decay is not an exhaustive explanation of blindness in the eyewitness context. 
Commentary on the effect of accuracy on blindness in eyewitness testimony In view of the counterintuitive findings concerning the role of memory and due to potential reservations as to the trust in null findings, we consider the following clarification essential. Our conclusion that forgetting fails to explain blindness does not imply that variations in memory strength are negligible. Indeed, long retention intervals which compromise memory strength are associated with increases in blindness rates (Chapters $3 \& 7$ ). It rather suggests that memory decay is unlikely to explain why people fail to notice blindness manipulations. This proposition is based on the facts that a) highly accurate participants are more often than not blind to manipulations and b) memory strength has time and again been proven not to be a predictor of detection (see Chapters 3-7). Yet, one might argue that the individual studies are not powerful enough to detect the effect of accuracy on blindness and thus our conclusion is farfetched. To address this concern we conducted a meta-analysis, using the Explanatory Software for Confidence Intervals (Cumming, 2012). For this purpose, the data of the four eyewitness identification experiments $(k=4)$ reported in Chapters 3-6 were entered, comprising $N=326$ participants. Our dependent variable was the number of blind participants and detectors. Our independent variable was participants' accuracy with regard to their identification decisions, which served as a proxy of memory strength. For the purpose of this comparison, we looked at the overall blindness rates regardless of participants' choosing behaviour (choosers vs. nonchoosers), lineup mode (target-present vs. target-absent) or ethnical background (African vs. European). Given the universal nature of memory, if memory strength were to affect blindness, the effect should be prominent for all participants. Our analysis yielded a small effect size with the confidence intervals including zero $(d=0.12,95 \% \mathrm{Cl}[-0.09$, $0.38]$ ), indicating once more no differences in blindness rates between accurate and inaccurate participants $(z=1.15, p=.248)$. Therefore, low accuracy is not a sine qua non for blindness to occur and it cannot account for the effect. Yet, we believe that memory decay and forgetting contributes to increase the ambiguity surrounding a decision, which in turn moderates blindness. However, as ambiguity is influenced by many other factors the impact of memory decay alone is rather small.

Commentary on choice blindness, misinformation, and post-identification feedback On a theoretical level, choice blindness, and blindness in the context of eyewitness testimony in particular, may seem equivalent to the misinformation effect (Loftus, 2005; Loftus \& Hoffman, 1989). In a typical misinformation paradigm participants are presented with misleading information about an event witnessed earlier. As a result of the presentation of misleading information memory is affected in such a way that 
participants recall information that they never witnessed. Likewise, in a choice blindness paradigm the manipulated outcome may serve as the misleading information that affects the memory of the original decision, hence preventing participants from detecting the manipulated outcome. Furthermore, post-identification feedback seems to be the key term when one tries to explain why in Chapter 4 our manipulations reduced post-identification confidence. Earlier research has shown that confirming and disconfirming feedback, among others, can cause witnesses to be more (or less) confident in their identification decisions (Douglass \& Steblay, 2006; Steblay, Wells, \& Douglass, 2014; Wells \& Bradfield, 1998). Furthermore, post-identification feedback, in general, is known to shape witnesses' meta-cognitive beliefs. This includes estimates regarding the viewing conditions and the distance to the target (Bradfield et al., 2002; Douglass \& Steblay, 2006; Palmer, Brewer, \& Weber, 2010; Steblay et al., 2014). Our pattern of results is in line with these research findings. Specifically, the manipulated outcome might have been perceived as a form of disconfirming feedback, resulting in participants being less confident in their identification decisions.

One might therefore argue that choice blindness is nothing but a new name for an old effect. In our point of view, however, choice blindness is a broader construct that integrates effects such as misinformation and post-identification feedback. As discussed in Chapter 3, the choice blindness procedure incorporates consequences of the manipulation and consequences of blindness. Therefore, we speculate that the manipulation contaminates eyewitness estimates for implicit measures in a similar way to post-identification feedback. Choice blindness per se, however, might be equivalent to the behavioural effects of internalized misinformation (Merckelbach et al., 2011a), where the presentation of the manipulated outcome could lead participants to develop detailed recollections of deciding for that outcome. Whereas the finding of participants confabulating reasons for their non-choices (Hall et al., 2010; Johansson et al., 2005) provides support for this view, our observation that participants can recall their original choice with reasonable precision (Chapter 9) speaks for a segregation of blindness and post-identification feedback. Additionally, one should consider that misinformation includes suggestions about a witnessed event (i.e., passive act) and not about a deliberate decision for which individuals need to evaluate the strength of their memory (i.e., active act) as in the choice blindness paradigm. Taken together, we have reasons to believe that choice blindness and misinformation may not be as closely related as initially thought. Nevertheless, future research is required to establish the precise link between the two phenomena.

Based on the findings of the present dissertation it would be hard to conclude on the mechanism underlying choice blindness. Nonetheless, our findings provide interesting insights for understanding choice blindness as they rule out potential mechanisms as memory decay. Yet the question remains: what are the underpinnings 
of choice blindness? This question deserves systematic research attention. However, to grasp the fundamentals of choice blindness, it may be wiser to consider first why blindness occurs at all in everyday life.

The "what the hell is it for?" question

On the pursuit of a reasonable explanation on why choice blindness originate in everyday life, one can speculate that the effect reflects a mechanism of adaptive behaviour aimed at protecting individuals from psychological or physical damage. For example the pioneers of choice blindness, , Johansson et al. (2011) argued that people have limited access to the reasons for their actions (cf. Nisbett \& Wilson, 1977). As a consequence, they can come to like what they are told or suggested to like. The endorsement of the suggested, instead of the original, choices may generate a degree of cognitive dissonance, which people want to overcome. Hence, convince themselves that they, indeed, prefer the manipulated outcome. Therefore, Johansson and Hall believed choice blindness to be a mechanism useful for reducing cognitive dissonance (Festinger, 1962; Henkel \& Mather, 2007).

Additionally, one may assume that choice blindness can be attributed to memory distrust. Memory distrust refers to the disbelief individuals develop about the accuracy of their memory, which makes them more susceptible to accepting information suggested by external sources (Gudjonsson, Kopelman, \& MacKeith, 1999; Gudjonsson, Sigurdsson, Sigurdardottir, Steinthorsson, \& Sigurdardottir, 2014). In a state of memory distrust individuals have difficulty in differentiating whether new information was generated internally or externally, which makes them prone to accept misleading information (van Bergen, Horselenberg, Merckelbach, Jelicic, \& Beckers, 2010). This mechanism may help to prevent confrontation and thus exposure to conflict and to possible threatening situations. Hence, choice blindness may reflect participants' mistrust in their memory for details that were either not well preserved or for which they did not feel confident about.

A different approach to this discussion is to consider the possibility that choice blindness is a maladaptive mechanism. From an evolutionary perspective and considering the role of natural selection in erasing maladaptive traits, the argument may at first appear weak; the idea should not be easily dismissed. The rapid move from the industrial age to the information age resulted in a complex environment for which individuals' mental capabilities may not have adjusted yet. For example, to reduce cognitive load the human mind assumes a relative stability and consistency in the external world. Indeed, this assumption may have been suitable for times prior to the industrial revolution. In the information age, however, the assumption of a stable

$7 \quad$ The phrase belongs to Alan Baddeley (1988) 
environment may hinder instead of facilitate cognitive performance. That is because to achieve the assumption of stability one should simply never reflect on reasons for doubting this stability. Therefore, choice blindness may be an error in information processing as a result of the new taxing environment or a by-product of other adaptive cognitive process. Similar approaches have been suggested for the usefulness of illusory contours (Grossberg \& Mingolla, 1985, 1987) and the evolutionary utility of aging (Fabian \& Flatt, 2011). For example, according to some theorists aging is a maladaptive by-product of selection for increased fitness early in life. Analogously, choice blindness may be the maladaptive by-product of logical reasoning (i.e., capacity for consciously making sense of the world and justifying practices; Kompridis, 2000). Although constructing experiments to explore this assumption is a difficult endeavour, this is a research avenue worth of exploration.

\section{Practical Implications}

The research findings presented in this dissertation have important implications for law enforcement agencies and legal professionals. As the cases of Bernard Maughan (Chapter 3; Wolchover, n.d.) and Herman Atkins (Chapter 7; Herman Atkins, 2015) exemplify, a situation in which a lineup administrator could mistakenly write down the wrong identification decision or temper with a witness statement may not be that unrealistic. Clearly, inadvertent or deliberate manipulations even of minor details can impede the accurate reconstruction of an event and possibly incriminate innocent suspects. Importantly, the present findings indicate that a large proportion of eyewitness fail to detect such mistakes.

Nevertheless, one may object that the records of identification decisions are unlikely to be incorrect or that witnesses usually sign their names next to the photo of the identified suspect. Considering how often professionals make procedural errors (e.g., erroneous matches of bullets, foot- and fingerprints; Saks \& Koehler, 2005) and the impact of biases due to contextual influences in the forensic science (Kassin et al., 2013), we posit that such a scenario is not at all that unrealistic. The report on prosecutorial misconduct cases compiled by the Northern California Innocence Project sides with the idea of altered identification decisions and tempered with testimony in real investigations (NCIP; Ridolfi \& Possley, 2010). The report reveals 4,000 cases of alleged misconduct, in 707 of which the courts explicitly established that the prosecutors deliberately mishandled, mistreated or destroyed evidence. Thus, errors in the recording of eyewitness decisions do occur it would be naïve to think otherwise.

Apart from the inability of witnesses to detect alterations in their testimony, the present work also speaks to the issue of extended delays between the police investigation and the time until cases reach the courts. Even under the speedy trial act as applied in the US judicial system trials begin the earliest 90-120 days after the arrest 
of the suspect (Shermer et al., 2011). This is worrying considering the increasing blindness rates with longer delays (Chapters $3 \& 7$ ). The findings of the present dissertation suggest that by the time witnesses are questioned in court, a large proportion would fail to realise that they are sitting in front of the wrong defendant or that their statements contain inaccuracies.

The issues emerging from our line of work directly appeal to and emphasize the importance of blind lineup administration procedures that leave little room for surreptitious manipulations. Specifically, the lineup should be administered by an officer who is not involved in the case and does not know who the suspect is. For example, recent findings suggest that lineup administrators' knowledge about the position of a suspect in the lineup affects the lineup outcome in accordance with the expectancies of the administrator (Rodriguez \& Berry, 2014). If this is not possible the witness should be instructed that the administrator does not know who the suspect is (Wells et al., 1998). Additionally, the findings of Chapter 7 highlight the importance of camera recordings during interviewing. This is particularly important in light of findings indicating the relevance of blindness phenomena in the field of interrogations and false confessions (Sauerland et al., 2015; Sauerland, Schell, et al., 2013). Finally, the present work stresses the importance of Smalarz and Wells' (2015) proposal to metaphorically interpret eyewitness memory as a form of trace evidence (i.e., physical trace left by the culprit at the crime scene from which we can establish the culprit's identity). By treating eyewitness memory like physical trace evidence, we achieve to put emphasis on the risk of contamination and thus on the importance of clear protocols in capturing, preserving and interpreting eyewitness testimony evidence. To conclude, the vast majority of these suggested amendments to the judicial procedures are inexpensive, or even free of costs, and could be easily applied to most countries. As long as there is the will for policy change and proper training those suggestions would amend criminal justice practices.

\section{Methodological Remarks and Future Directions}

Although the studies presented in this dissertation have shown interesting and relevant findings, we need to address methodological shortcomings. First we would like to discuss the issue of power in our studies. Our experimental designs often required the division of the original sample into accurate and inaccurate participants or choosers and nonchoosers. These variables, however, were determined post-hoc as a result of participants' behaviour during the identification or recall task. Hence, we were often confronted with small subsamples that impose restrictions on the complexity of the analyses (e.g., Chapters $5 \& 6$ ). As a result, we were forced to almost exclusively examine main effects and disregard possible interactions. Although there are no means to guarantee adequate distribution of participants across categories, the use 
of larger samples sizes may be a remedy for this type of problem. The reason why we did not use larger sample sizes is that to achieve the adequate size we would have to resort to online experiments. These, however, come with limited ecological validity and control of the testing procedure. Had we used such methods we could not have excluded the possibility that the results were due to participants' lack of attention to the task. Therefore, we opted for strict control rather than large sample sizes. This is not to imply that we should not strive for statistical power. Therefore, we welcome replications of the present studies using larger sample sizes.

A second shortcoming is the valence of the employed crime scenarios. To stay in the realm of ethics, both our laboratory and field studies, participants were confronted with minor, non-violent offences. If participants were to witness more emotional crimes, the results might have been different. Emotional scenes are known to be encoded better and processed more deeply than non-emotional scenes (Christianson, 1992; Christianson \& Loftus, 1987; LaBar \& Cabeza, 2006). Under such circumstances participants may be more resistant to blindness manipulations. Therefore, we encourage other researchers to examine the link between emotional valence and blindness in the context of eyewitness testimony.

In spite of the aforementioned shortcomings, we believe that the present dissertation yielded new insights in the field of choice blindness and particularly for blindness in the eyewitness context. We believe that the systematic investigation of differences in susceptibility to blindness between choosers and nonchoosers and of the role of memory strength for blindness in the context of eyewitness testimony is an interesting area of exploration. This line of research will not only provide useful insights for the decision making of eyewitnesses but also speak to the mechanism of choice blindness.

Furthermore, we would like to encourage research investigating the long term effects of blindness manipulations in eyewitness testimony. Next to the findings showing that blindness manipulations can have long lasting effects in the context of preferences (Johansson et al., 2013) and false confessions (Sauerland, Schell, et al., 2013), preliminary evidence indicates that blind participants are more likely to shift their identification responses to match the manipulated outcome when they are asked to perform the same identification task a second time (Cochran \& Greenspan, personal communication, $1^{\text {st }}$ March 2015). This is of practical relevance as it would indicate that eyewitnesses, next to failing to notice a manipulation, would continue to identify the innocent suspect as the perpetrator in consecutive identification incidents, as for example, at trial. Hence the error would prevail across the different legal stages. Finally, given the severe consequences blindness manipulations have for legal practice, another field worth exploring concerns methods of eradicating blindness. Drawing from the misinformation literature and the use of warnings to eliminate or reverse the 
misinformation effect (Oeberst \& Blank, 2012; Zaragoza et al., 2006), future studies could elaborate on the use of similar warnings to prevent or reverse blindness in the eyewitness context.

\section{CONCLUDING REMARKS}

The fact that witnesses can be blind to surreptitious manipulations on their identification decisions and witness statements may daunt researchers dealing with eyewitness memory and decision making. Certainly, the findings of the present dissertation challenge the view of humans as deliberate agents and raise questions about the decision making of eyewitnesses and the functioning of memory. Precisely because choice blindness attacks the notion of human rationality our work has not escaped criticism. Apart from concerns regarding the methodological sanctity, the criticism often pertains to the relevance of choice blindness for the legal setting. Despite evidence to the opposite, many remain unconvinced and transform the argument to incorporate the frequency of blindness phenomena in the legal setting. That is, although there may be cases of blindness these are sparse. Therefore, why should we devote energy and resources into an effect that is, at least, rare? Until now, we are not in a position to estimate the exact magnitude of the effect in real cases and research is needed in that direction. However, we consider the question in its essence moral. To answer the question one should consider one's personal moral compass to determine for him or herself how many cases of innocent people incarcerated as a result of blindness constitute a problem worth attention. To conclude, we trust that we have demonstrated that research on choice blindness in the context of eyewitness testimony is critical for the legal practice and we hope to see more research in this field in the near future. 

Summary 



\section{SUMMARY}

The present dissertation aims to investigate the effect of choice blindness in the eyewitness context. Choice blindness refers to the difficulty people have in detecting manipulations in their own previously made choices. In a typical choice blindness task participants first indicate a preference. Then, they are presented either with their original response (non-manipulated trial) or with a response that is inconsistent with their own (manipulated trial) and they are asked to explain the reasons for their choice. Finally, participants are informed about the possibility of a manipulation and are asked to indicate if that was true for them. The typical finding is that the overwhelming majority of participants remain unaware to this surreptitious change in the outcome of their decision and accept as their own a decision they never made. Inspired by this intriguing effect in the present dissertation we aimed to: 1) establish the relevance of choice blindness in eyewitness testimony, 2) explore the moderating factors of the effect of blindness in eyewitness testimony, and 3) unravel the mechanism underlying choice blindness. To that end, we modified the choice blindness paradigm to incorporate manipulations of eyewitness identification decisions and witness statements.

In Part 1 of this dissertation, we focused on establishing the occurrence of choice blindness in earwitnesses and eyewitnesses. Examining the effect of blindness manipulations on earwitnesses in Chapter 2, we found that choice blindness transfers to auditory stimuli, with the overwhelming majority of participants being blind to our manipulations (71-81\%). Following the successful application of blindness manipulations for auditory stimuli, in Chapter 3 we aimed to identify the conditions under which blindness occurs in facial recognition decisions. In five Experiments, participants watched four mock crime videos and made choices that were either evaluative (i.e., choice based on sympathy; Experiment 1) or absolute in nature (i.e., identification decision; Experiments $2 a-c, 3)$. Our hypothesis that in an eyewitness setting, choice blindness rates would depend on decision type (evaluative vs. absolute) was only partially supported (Experiment 1). In Experiments 2a-c, were we employed simple recognition procedures, we found virtually no or modest blindness rates (0-35\%) in student and non-student samples. To increase the ecological validity of our findings, in Experiment 3 we inserted a 48 hrs interval between the presentation of the recognition task and the presentation of the manipulated outcome. Under these conditions blindness rates increased dramatically (39.4-68.3\%) compared with the previous three recognition experiments.

The second part (Part 2) of the dissertation is devoted to establishing the occurrence of the effect under naturalistic encoding conditions; hence affirming its relevance for the judicial system. Therefore, we conducted two additional experiments employing a 
field study methodology. Using staged non-criminal (Chapter 4) and criminal (Chapter 5) events, we replicated our laboratory findings and showed that blindness for identification decisions can occur at an alarmingly high level in real life settings (41-69\%). Moreover, we extended our knowledge by demonstrating that blindness phenomena occur for choosers and, to a lesser extent, nonchoosers, as well as for target-present and, to a lesser extent, target-absent lineups.

In light of the stable nature of blindness for identification decisions, in Part 3 we aimed to investigate whether blindness could be relevant for other aspects of eyewitness testimony. In Chapter 6 we examined whether the increased cognitive effort that is inherent in other-race identifications would increase blindness rates for othercompared with own-race identifications. Indeed, participants who made other-race identifications were more likely to be blind to the manipulations than participants who made own-race identifications. Therefore, these findings indicate that some estimator variables can facilitate blindness. In Chapter 7, in a series of three studies, we examined the ability of eyewitnesses to detect manipulations introduced to their written statements. Results revealed that although blindness varied as a function of the delay between giving testimony and being presented with the manipulated statement, a substantial proportion of manipulations in eyewitnesses' written statements went undetected even within minutes after giving testimony.

Finally, in Part 4 we attempted to understand the mechanism behind choice blindness. In Chapter 8 we focused on the role of self-relevance as a moderator, but found only weak support for the hypothesis that self-relevance can decrease blindness rates. In Chapter 9, we investigated memory decay as a potential candidate. We hypothesized that the presentation of the manipulated outcome should hinder the recollection of the memory trace of the original choice. Contrary to our assumption, participants were able to recall their original choice with reasonable precision (recall accuracy: $46.2 \%-85.4 \%$ ). These results provide interesting insights for understanding choice blindness, as they rule out potential mechanisms as memory decay. However, based on the findings of the present dissertation it would be hard to conclude on the mechanism underlying choice blindness. Nonetheless, we suggest that we can draw firm conclusions about the moderators of blindness in the eyewitness context. In fact, we believe that ambiguity is the key moderator. That is, ambiguous decisions result in participants being less likely to detect the discrepancy between the original choice and the manipulated outcome. In the context of eyewitness testimony, the longer retention interval as well as the heightened similarity between the original choice and the presentation of the manipulate outcome and potentially memory decay can be conceptualized as factors that increase the ambiguity surrounding a decision, which in turn increases blindness rates. 
The present findings indicate that a large proportion of eyewitness fail to detect mistakes and changes in their identification decisions and witness statements. Clearly, mistaken or deliberate manipulations even of minor details can impede the accurate reconstruction of an event and possibly incriminate innocent suspects. Therefore, the issues emerging from our line of work directly appeal to and emphasize the importance of blind lineup administration procedures that leave little room for surreptitious manipulations. Additionally, our findings underscore the importance of camera recordings during identification and interviewing.

To conclude, the fact that witnesses can be blind to surreptitious manipulations on their identification decisions and witness statements may daunt researchers dealing with eyewitness memory and decision making. Indeed, the findings of the present dissertation challenge the view of humans as deliberate agents and raise questions about decision making. Yet, we believe we have demonstrated that research on blindness phenomena is critical for the legal practice and we hope to see more research in this field in the near future. 

$\Sigma u ́ v o \psi n$ 



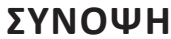

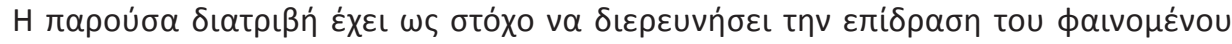

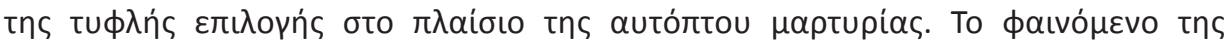

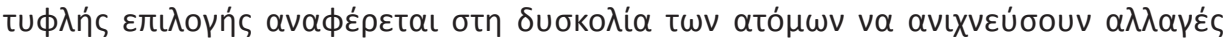

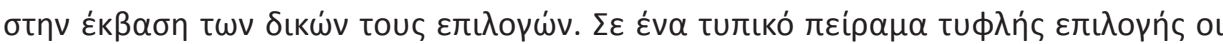

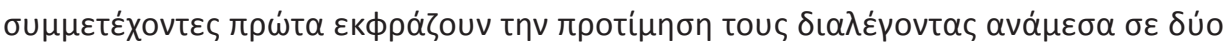

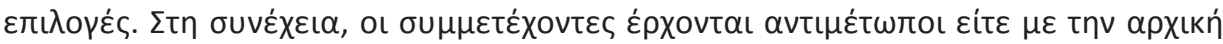

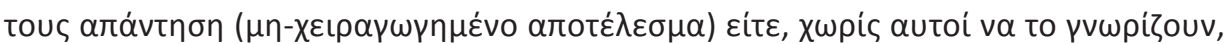

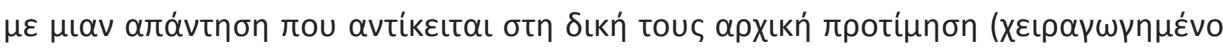

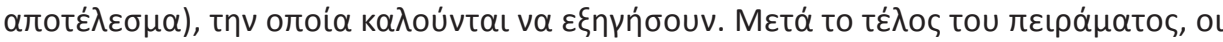

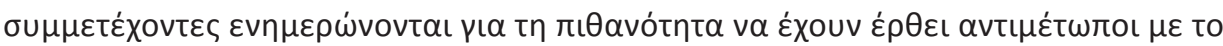

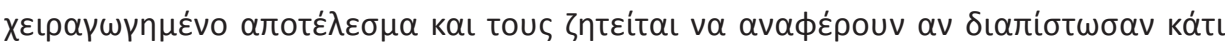

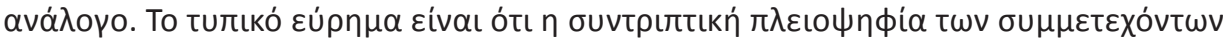

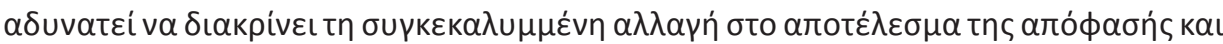

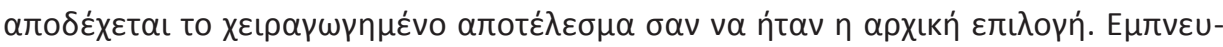

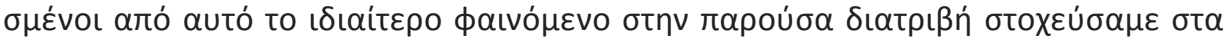

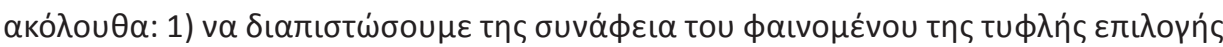

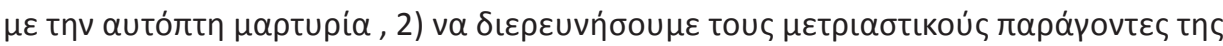

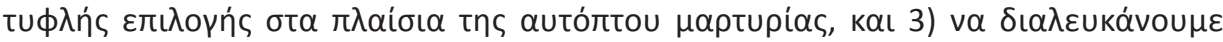

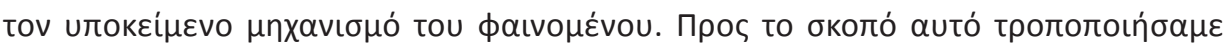

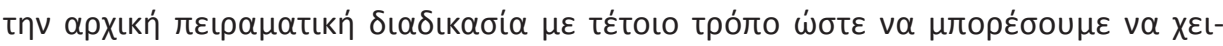

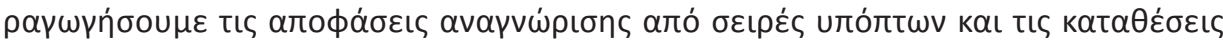

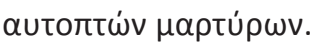

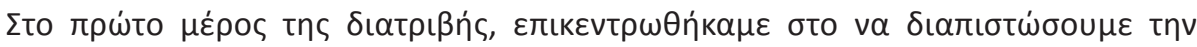

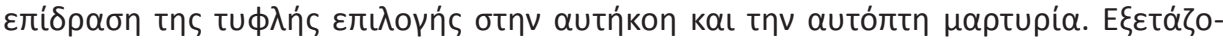

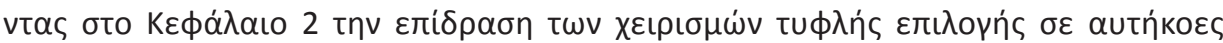

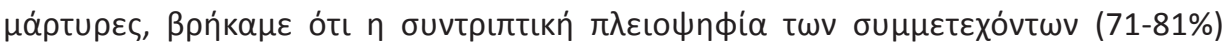

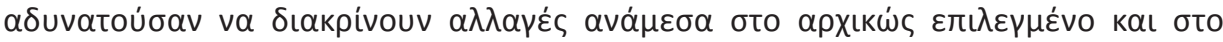

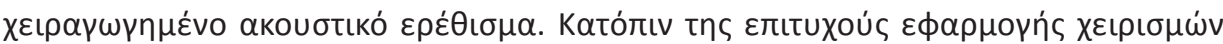

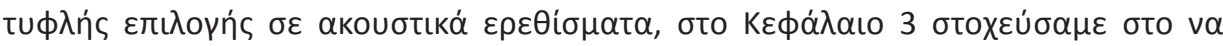

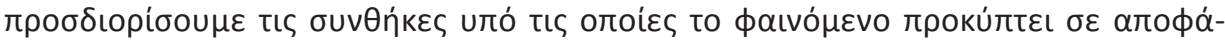

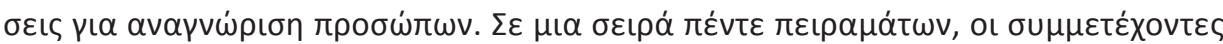

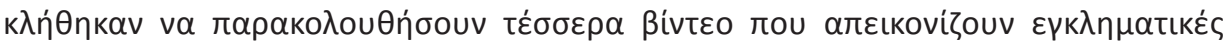

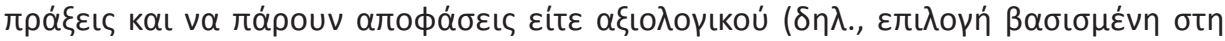

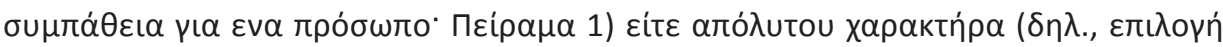

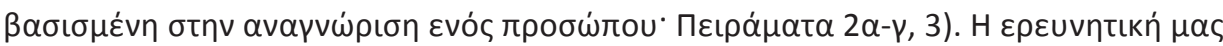

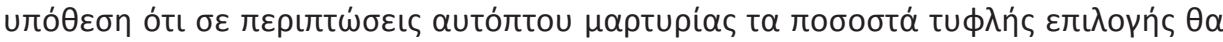

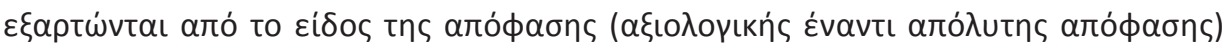




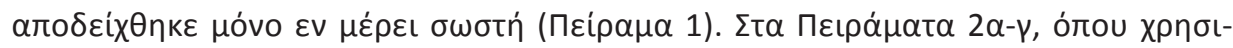

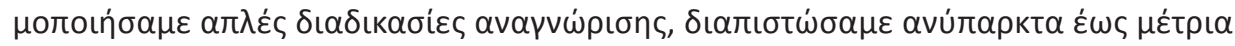

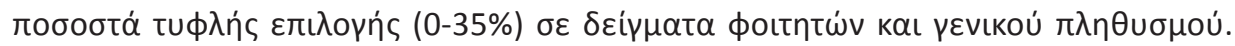

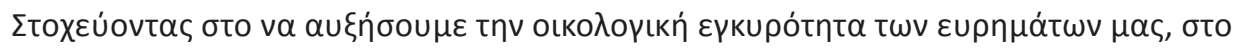

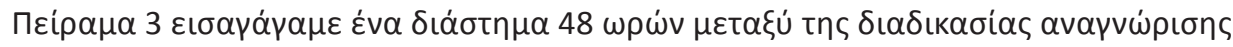

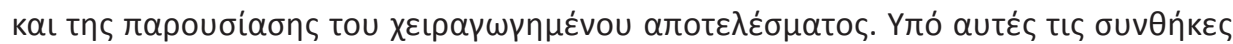

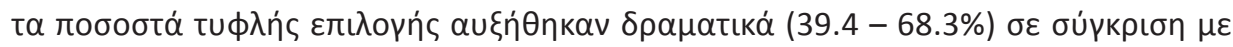

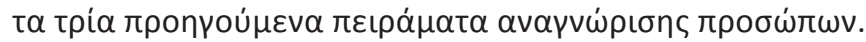

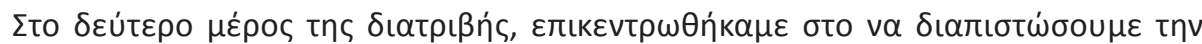

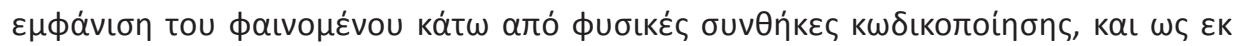

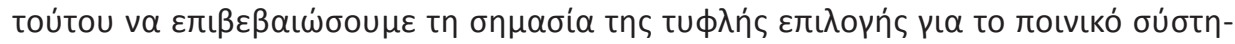

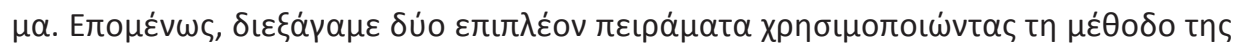

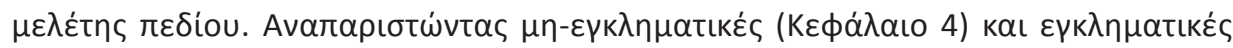

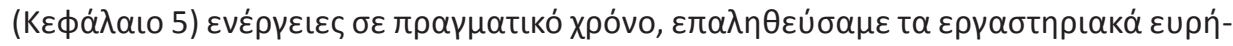

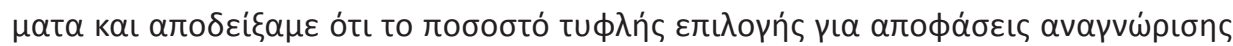

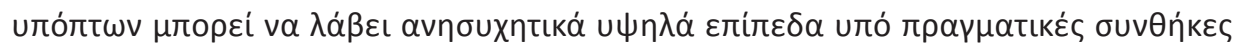

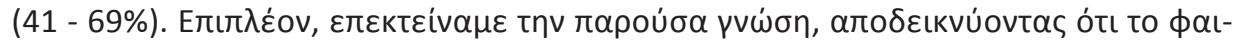

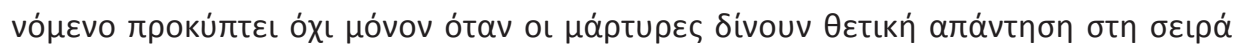

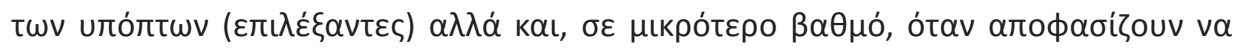

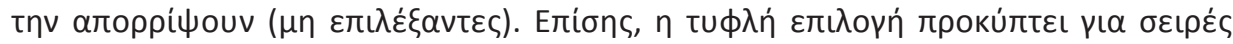

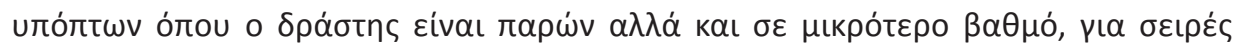

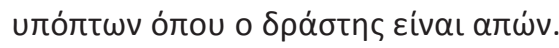

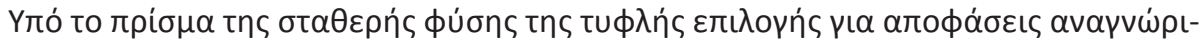

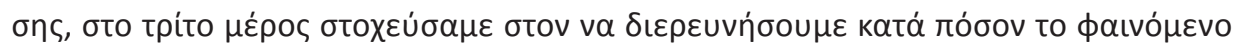

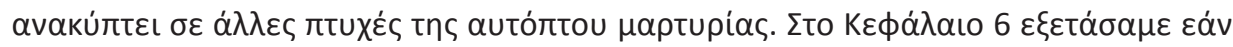

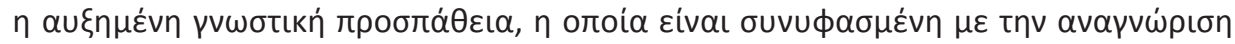

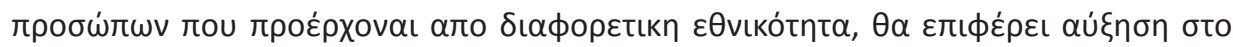

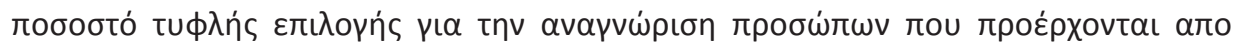

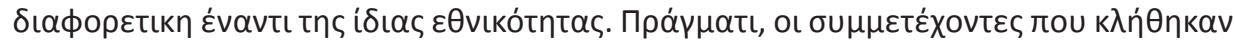

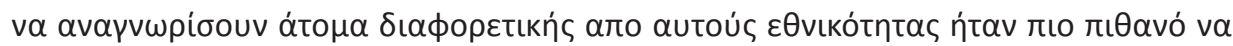

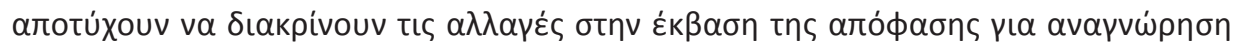

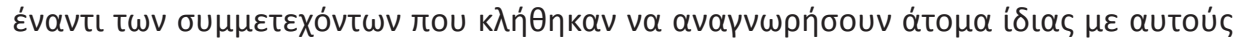

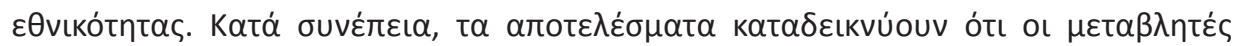

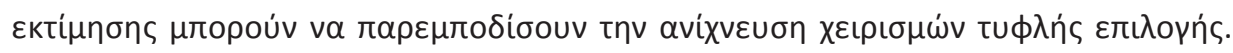

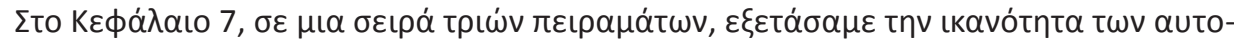

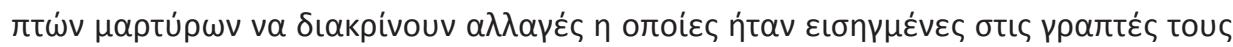

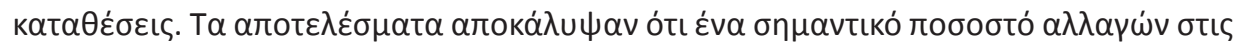

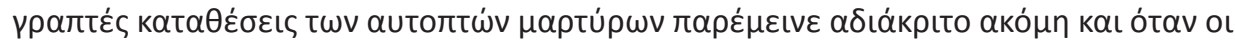

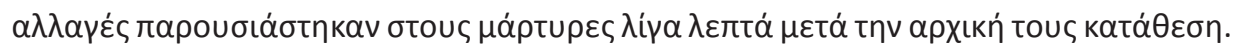




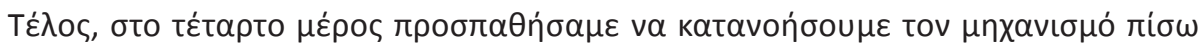

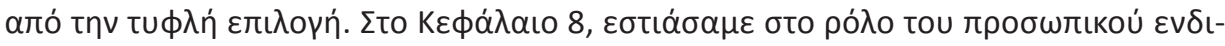

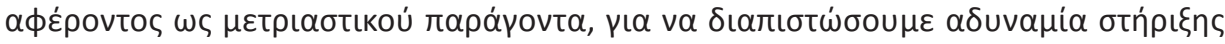

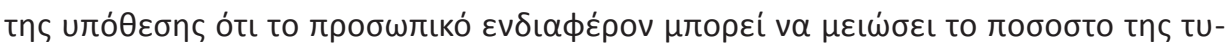

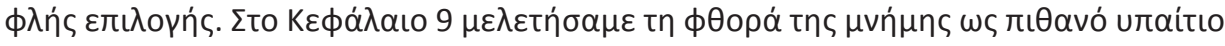

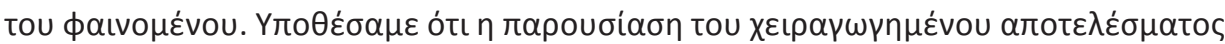

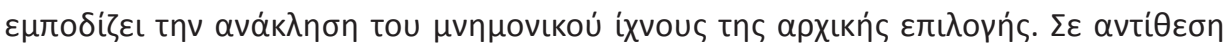

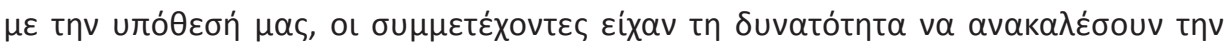

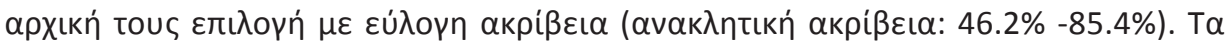

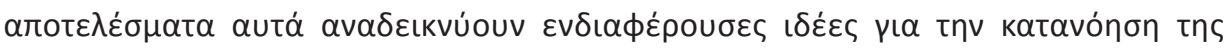

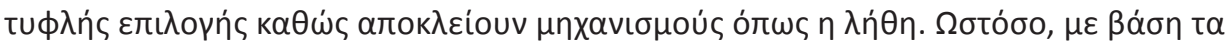

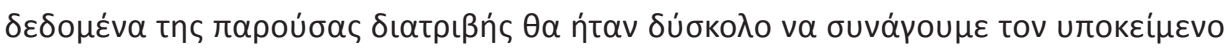

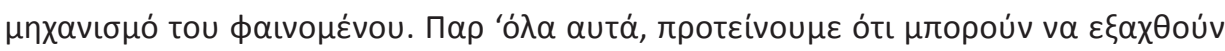

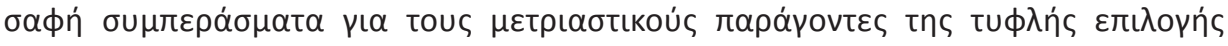

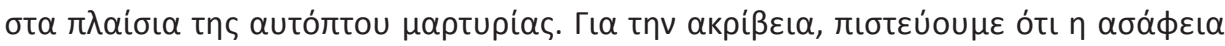

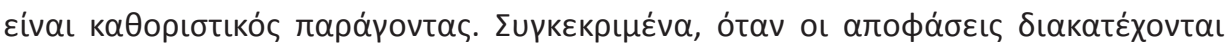

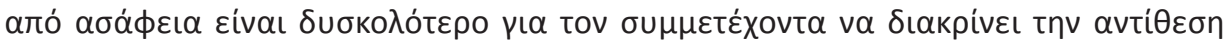

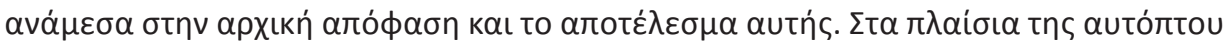

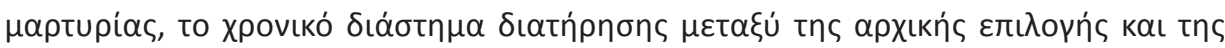

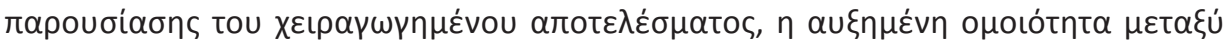

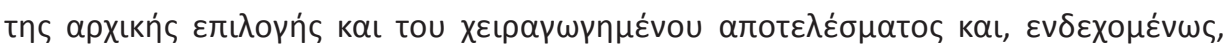

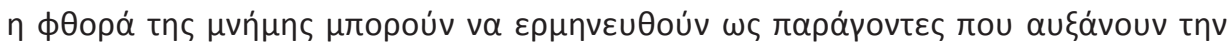

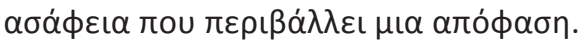

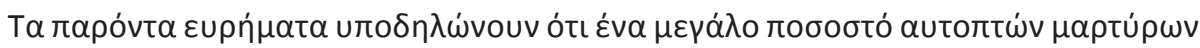

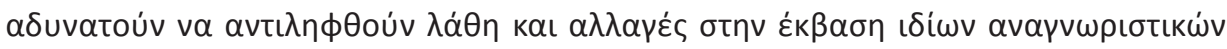

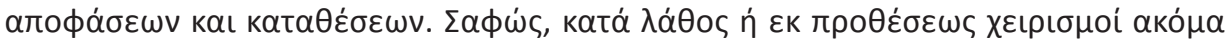

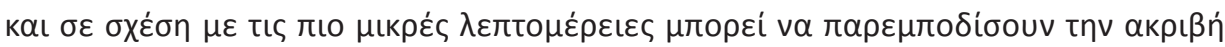

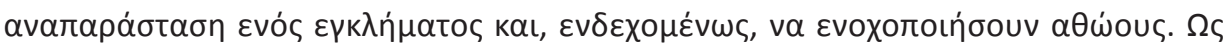

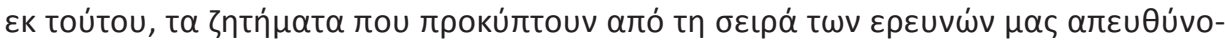

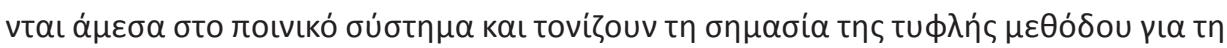

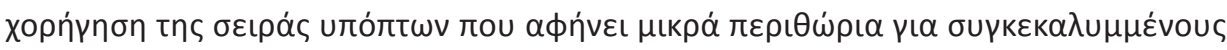

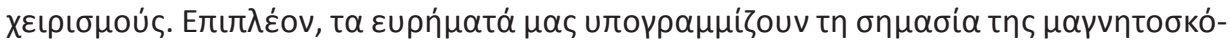

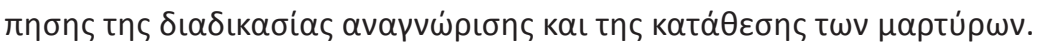

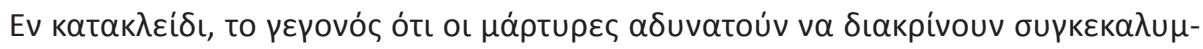

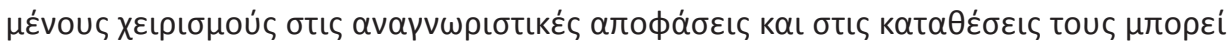

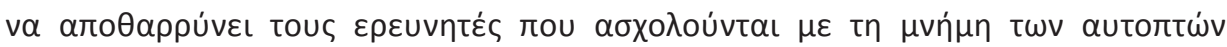

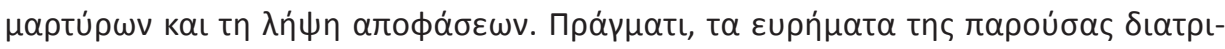

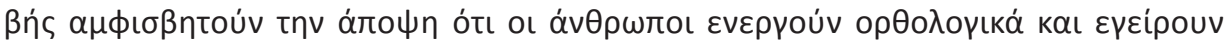

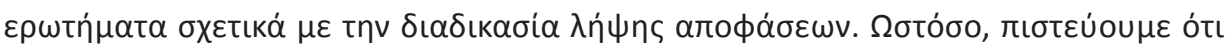




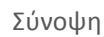

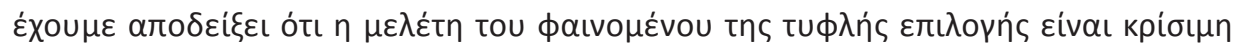

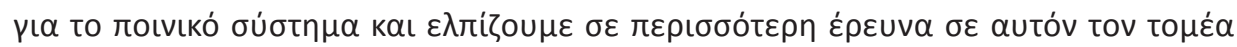

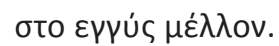




\section{REFERENCES}

Aardema, F., Johansson, P., Hall, L., Paradisis, S.-M., Zidani, M., \& Roberts, S. (2014). Choice blindness, confabulatory introspection, and obsessive-compulsive symptoms: A new area of investigation. International Journal of Cognitive Therapy, 7, 83-102. doi:10.1521/ ijct.2014.7.1.83

Adams Jr, R. B., Pauker, K., \& Weisbuch, M. (2010). Looking the other way: The role of gaze direction in the cross-race memory effect. Journal of Experimental Social Psychology, 46, 478-481. doi:10.1016/j.jesp.2009.12.016

Anthony, T., Copper, C., \& Mullen, B. (1992). Cross-racial facial identification: A social cognitive integration. Personality and Social Psychology Bulletin, 296-301. doi:0.1177/0146167292183005

Arnold, M. M. (2013). Monitoring and meta-metacognition in the own-race bias. Acta Psychologica, 144, 380-389. doi:10.1016/j.actpsy.2013.07.007

Ayers, M. S., \& Reder, L. M. (1998). A theoretical review of the misinformation effect: Predictions from an activation-based memory model. Psychonomic Bulletin and Review, 5, 1-21. doi:10.3758/bf03209454

Baddeley, A. (1988). But what the hell is it for? In M. M. Gruneberg, P. E. Morris \& R. N. Sykes (Eds.), Practical aspects of memory: Current research and issues: Vol. 1: Memory in everyday life (pp. 3-18). New York: John Wiley \& Sons.

Barclay, C. R., \& Wellman, H. M. (1986). Accuracies and inaccuracies in autobiographical memories. Journal of Memory and Language, 25, 93-103. doi:10.1016/0749-596X(86)90023-9

Bargh, J. A. (1982). Attention and automaticity in the processing of self-relevant information. Journal of Personality and Social Psychology, 43, 425-436. doi:10.1037/0022-3514.43.3.425

Barkowitz, P., \& Brigham, J. C. (1982). Recognition of faces: Own-race bias, incentive, and time delay. Journal of Applied Social Psychology, 12, 255-268. doi:10.1111/j.1559-1816.1982. tb00863.x

Bassili, J. N. (1996). Meta-judgmental versus operative indexes of psychological attributes: The case of measures of attitude strength. Journal of Personality and Social Psychology, 71, 637-653. doi:10.1037/0022-3514.71.4.637

Behrman, B. W., \& Davey, S. L. (2001). Eyewitness identification in actual criminal cases: An archival analysis. Law and Human Behavior, 25, 475-491. doi:10.1023/A:1012840831846

Belli, R. F., Lindsay, D. S., Gales, M. S., \& McCarthy, T. T. (1994). Memory impairment and source misattribution in postevent misinformation experiments with short retention intervals. Memory \& Cognition, 22, 40-54. doi:10.3758/BF03202760

Belli, R. F., Windschitl, P. D., McCarthy, T. T., \& Winfrey, S. E. (1992). Detecting memory impairment with a modified test procedure: Manipulating retention interval with centrally presented event items. Journal of Experimental Psychology: Learning, Memory, and Cognition, 18, 356-367. doi:10.1037/0278-7393.18.2.356 
Block, S. D., Greenberg, S. N., \& Goodman, G. S. (2009). Remembrance of eyewitness testimony: Effects of emotional content, self-relevance, and emotional tone. Journal of Applied Social Psychology, 39, 2859-2878. doi:10.1111/j.1559-1816.2009.00553.x

Bohner, G., \& Dickel, N. (2011). Attitudes and attitude change. Annual Review of Psychology, 62, 391-417. doi:10.1146/annurev.psych.121208.131609

Bradfield, A. L., Wells, G. L., \& Olson, E. A. (2002). The damaging effect of confirming feedback on the relation between eyewitness certainty and identification accuracy. Journal of Applied Psychology, 87, 112-120. doi:10.1037/0021-9010.87.1.112

Brainerd, C. J., Reyna, V. F., Howe, M. L., Kingma, J., \& Guttentag, R. E. (1990). The development of forgetting and reminiscence. Monographs of the Society for Research in Child Development, 55, 100-109. doi:10.2307/1166106

Brédart, S., Barsics, C., \& Hanley, R. (2009). Recalling semantic information about personally known faces and voices. European Journal of Cognitive Psychology, 21, 1013-1021.

Brehm, J. W. (1956). Postdecision changes in the desirability of alternatives. The Journal of Abnormal and Social Psychology, 52, 384-389. doi:10.1037/h0041006

Brewer, N., Keast, A., \& Rishworth, A. (2002). The confidence-accuracy relationship in eyewitness identification: The effects of reflection and disconfirmation on correlation and calibration. Journal of Experimental Psychology: Applied, 8, 44-56. doi:10.1037/1076-898X.8.1.44

Brewer, N., Weber, N., \& Semmler, C. (2007). A role of theory in eyewitness identification research In R. C. L. Lindsay, D. F. Ross, J. D. Read \& M. P. Toglia (Eds.), The handbook of eyewitness psychology, Vol II: Memory for people. (pp. 201-218). Mahwah, NJ US: Lawrence Erlbaum Associates Publishers.

Brewer, N., \& Wells, G. L. (2006). The confidence-accuracy relationship in eyewitness identification: Effects of lineup instructions, foil similarity, and target-absent base rates. Journal of Experimental Psychology: Applied, 12, 11-30. doi:10.1037/1076-898X.12.1.11

Brigham, J. C., \& Barkowitz, P. (1978). Do "They all look alike?" The effect of race, sex, experience, and attitudes on the ability to recognize faces. Journal of Applied Social Psychology, 8, 306-318. doi:10.1111/j.1559-1816.1978.tb00786.x

Brigham, J. C., \& Cairns, D. L. (1988). The effect of mugshot inspections on eyewitness identification accuracy. Journal of Applied Social Psychology, 18, 1394-1410. doi:10.1111/j.1559-1816.1988.tb01214.x

Brigham, J. C., \& Malpass, R. S. (1985). The role of experience and contact in the recognition of faces of own- and other-race persons. Journal of Social Issues, 41, 139-155. doi:10.1111/j.1540-4560.1985.tb01133.x

Brown, J. M. (2003). Eyewitness memory for arousing events: Putting things into context. Applied Cognitive Psychology, 17, 93-106. doi:10.1002/acp.848

Brown, R., \& Kulik, J. (1977). Flashbulb memories. Cognition, 5, 73-99. doi:10.1016/00100277(77)90018-x 
Bruce, V., Burton, M. A., \& Dench, N. (1994). What's distinctive about a distinctive face? The Quarterly Journal of Experimental Psychology Section A: Human Experimental Psychology, 47, 119-141. doi:10.1080/14640749408401146

Bruck, M., \& Ceci, S. J. (1999). The suggestibility of children's memory. Annual Review of Psychology, 50, 419-439. doi:10.1146/annurev.psych.50.1.419

Buchanan, T. W. (2007). Retrieval of emotional memories. Psychological Bulletin, 133, 761-779. doi:10.1037/0033-2909.133.5.761

Ceci, S. J., \& Bruck, M. (1993). Suggestibility of the child witness: A historical review and synthesis. Psychological Bulletin, 113, 403-439. doi:10.1037/0033-2909.113.3.403

Chabris, C. F., \& Simons, D. (2010). The invisible gorilla and other ways our intuition deceives us. London, UK: HarperCollins

Charman, S., \& Wells, G. L. (2007). Applied lineup theory. In R. C. L. Lindsay, D. F. Ross, J. D. Read \& M. P. Toglia (Eds.), The handbook of eyewitness psychology, Vol II: Memory for people. (pp. 219-254). Mahwah, NJ US: Lawrence Erlbaum Associates Publishers.

Charman, S. D., \& Wells, G. L. (2012). The moderating effect of ecphoric experience on postidentification feedback: A critical test of the cues-based inference conceptualization. Applied Cognitive Psychology, 26, 243-250. doi:10.1002/acp.1815

Chiroro, P., \& Valentine, T. (1995). An investigation of the contact hypothesis of the own-race bias in face recognition. The Quarterly Journal of Experimental Psychology A: Human Experimental Psychology, 48A, 879-894. doi:10.1080/14640749508401421

Christianson, S. A. (1992). Emotional stress and eyewitness memory: A critical review. Psychological Bulletin, 112, 284-309. doi:10.1037/0033-2909.112.2.284

Christianson, S. A., Engelberg, E., \& Gustafson, A. (2007). Recognition of previous eyewitness testimony from an altered interrogation protocol: Potential effects of distortions. Psychology, Crime \& Law, 13, 583-589. doi:10.1080/10683160601095230

Christianson, S. A., \& Loftus, E. F. (1987). Memory for traumatic events. Applied Cognitive Psychology, 1, 225-239. doi:10.1002/acp.2350010402

Christianson, S. A., \& Loftus, E. F. (1991). Remembering emotional events: The fate of detailed information. Cognition and Emotion, 5, 81-108. doi:10.1080/02699939108411027

Clark, S. E. (2005). A re-examination of the effects of biased lineup instructions in eyewitness identification. Law and Human Behavior, 29, 575-604. doi:10.1007/s10979-005-7121-1

Cohen, J. (1988). Statistical power analysis for the behavioral sciences. Hillsdale, New Jersey: Lawrence Erlbaum Associates.

Connecticut Jury Instructions - Criminal. (2012). Connecticut, US: State of Connecticut Judicial Branch Retrieved from http://www.jud.ct.gov/Jl/criminal/part2/2.4-2.htm

Crovitz, H. F., \& Schiffman, H. (2013). Frequency of episodic memories as a function of their age. Bulletin of the Psychonomic Society, 4, 517-518. doi:10.3758/BF03334277 
Crowne, D. P., \& Marlowe, D. (1960). A new scale of social desirability independent of psychopathology. Journal of Consulting Psychology, 24, 349-354. doi:10.1037/h0047358

Cumming, G. (2012). Understanding the new statistics: Effect sizes, confidence intervals, and meta-analysis. New York: Routledge.

Cunningham, S. J., Vergunst, F., Macrae, C. N., \& Turk, D. J. (2013). Exploring early self-referential memory effects through ownership. British Journal of Developmental Psychology, 31, 289301. doi:10.1111/bjdp.12005

Cutler, B. L., \& Penrod, S. D. (1995). Mistaken identification: The eyewitness, psychology and the law (1st Paperback Edition edition ed.). Cambridge ; New York: Cambridge University Press.

Dalton, A. L., \& Daneman, M. (2006). Social suggestibility to central and peripheral misinformation. Memory, 14, 486-501. doi:10.1080/09658210500495073

Damjanovic, L., \& Hanley, J. R. (2007). Recalling episodic and semantic information about famous faces and voices. Memory \& Cognition, 35, 1205-1210. doi:10.3758/BF03193594

Davis, D., Loftus, E. F., Vanous, S., \& Cucciare, M. (2008). 'Unconscious transference' can be an instance of 'change blindness'. Applied Cognitive Psychology, 22, 605-623. doi:10.1002/ acp.1395

Deffenbacher, K. A., Bornstein, B. H., Penrod, S. D., \& McGorty, E. K. (2004). A meta-analytic review of the effects of high stress on eyewitness memory. Law and Human Behavior, 28, 687-706. doi:10.1007/s10979-004-0565-x

Dellarosa, D., \& Bourne Jr, L. E. (1984). Decisions and memory: Differential retrievability of consistent and contradictory evidence. Journal of Verbal Learning and Verbal Behavior, 23, 669-682. doi:10.1016/s0022-5371(84)90410-9

Dempsey, J. L., \& Pozzulo, J. D. (2008). Identification accuracy of eyewitnesses for a multiple perpetrator crime: Examining the simultaneous and elimination lineup procedures. American Journal of Forensic Psychology, 26, 67-81.

Derogatis, L. R., Lipman, R. S., \& Covi, L. (1973). SCL-90:An outpatient psychiatric rating scalepreliminary report. Psychopharmacology Bulletin, 9, 13-28.

Detained without trial: Fair Trials International's response to the European Commissions Green Paper on detention. (2011), from http://ec.europa.eu/justice/newsroom/criminal/opinion/files/110510/fti_pre-trial_detention_report_en.pdf

DNA Exonerations Nationwide. (2013). Innocence Project Fact Sheets. Retrieved from Innocence Project website: http://www.innocenceproject.org/Content/DNA_Exonerations_Nationwide.php\#

Dodd, D. H., \& Bradshaw, J. M. (1980). Leading questions and memory: Pragmatic constraints. Journal of Verbal Learning and Verbal Behavior, 19, 695-704. doi:10.1016/500225371(80)90379-5

Dolcos, F., LaBar, K. S., \& Cabeza, R. (2005). Remembering one year later: Role of the amygdala and the medial temporal lobe memory system in retrieving emotional memories. Proceed- 
ings of the National Academy of Sciences of the United States of America, 102, 2626-2631. doi:10.1073/pnas.0409848102

Douglass, A. B., \& Steblay, N. (2006). Memory distortion in eyewitnesses: A meta-analysis of the post-identification feedback effect. Applied Cognitive Psychology, 20, 859-869. doi:10.1002/acp.1237

Dysart, J. E., Lindsay, R. C. L., Hammond, R., \& Dupuis, P. (2001). Mug shot exposure prior to lineup identification: Interference, transference, and commitment effects. Journal of Applied Psychology, 86, 1280-1284. doi:10.1037/0021-9010.86.6.1280

Egan, L. C., Bloom, P., \& Santos, L. R. (2010). Choice-induced preferences in the absence of choice: Evidence from a blind two choice paradigm with young children and capuchin monkeys. Journal of Experimental Social Psychology, 46, 204-207. doi:10.1016/j.jesp.2009.08.014

Fabian, D., \& Flatt, T. (2011). The Evolution of Aging. Nature Education Knowledge, 3. Retrieved from http://www.nature.com/scitable/knowledge/library/the-evolution-ofaging-23651151

Festinger, L. (1962). A theory of cognitive dissonance. Palo Alto, CA US: Stanford University Press.

Findley, K., \& Scott, M. (2006). The multiple dimensions of tunnel vision in criminal cases. Wisconsin Law Review, 2006, 291-398.

Fischhoff, B. (1975). Hindsight is not equal to foresight: The effect of outcome knowledge on judgment under uncertainty. Journal of Experimental Psychology: Human Perception and Performance, 1, 288-299. doi:10.1037/0096-1523.1.3.288

Fisher, G. (1967). The performance of male prisoners on the Marlowe-Crowne Social Desirability. Journal of Clinical Psychology, 23, 473-475.

Fletcher-Watson, S., Collis, J. M., Findlay, J. M., \& Leekam, S. R. (2009). The development of change blindness: children's attentional priorities whilst viewing naturalistic scenes. Developmental Science, 12, 438-445. doi:10.1111/j.1467-7687.2008.00784.x

Forster, K. I., \& Forster, J. C. (2003). DMDX: A Windows display program with millisecond accuracy. Behavior Research Methods, Instruments, \& Computers, 116- 124. doi:10.3758/ BF03195503

Forsyth, D. R., \& Wibberly, K. H. (1993). The self-reference effect: Demonstrating schematic processing in the classroom. Teaching of Psychology, 20, 237-238. doi:10.1207/ s15328023top2004_11

Frost, P., Ingraham, M., \& Wilson, B. (2002). Why misinformation is more likely to be recognised over time: A source monitoring account. Memory, 10, 179-185. doi:10.1080/09658210143000317

Gabbert, F., Hope, L., \& Fisher, R. P. (2009). Protecting eyewitness evidence: Examining the efficacy of a self-administered interview tool. Law and Human Behavior, 33, 298-307. doi:10.1007/s10979-008-9146-8 
Gabbert, F., Memon, A., Allan, K., \& Wright, D. B. (2004). Say it to my face: Examining the effects of socially encountered misinformation. Legal and Criminological Psychology, 9, 215-227. doi:10.1348/1355325041719428

Goldsmith, M., Koriat, A., \& Weinberg-Eliezer, A. (2002). Strategic regulation of grain size in memory reporting. Journal of Experimental Psychology. General, 131, 73-95.

Gorenstein, G. W., \& Ellsworth, P. C. (1980). Effect of choosing an incorrect photograph on a later identification by an eyewitness. Journal of Applied Psychology, 65, 616-622. doi:10.1037/0021-9010.65.5.616

Grabowski, J. (2007). The writing superiority effect in the verbal recall of knowledge: Sources and determinants. In M. Torrance, L. van Waes \& D. Galbraith (Eds.), Writing and cognition: Research and applications (Vol. 20, pp. 165-179). Amsterdam: Elsevier.

Greathouse, S. M., \& Kovera, M. B. (2009). Instruction bias and lineup presentation moderate the effects of administrator knowledge on eyewitness identification. Law and Human Behavior, 33, 70-82. doi:10.1007/s10979-008-9136-x

Grossberg, S., \& Mingolla, E. (1985). Neural dynamics of form perception: Boundary completion, illusory figures, and neon color spreading. Psychological Review, 92, 173-211. doi:10.1037/0033-295X.92.2.173

Grossberg, S., \& Mingolla, E. (1987). Neural dynamics of surface perception: Boundary webs, illuminants, and shape-from-shading. Computer Vision, Graphics, and Image Processing, 37, 116-165. doi:10.1016/S0734-189X(87)80015-4

Gudjonsson, G. H. (1989). Compliance in an interrogative situation: A new scale. Personality and Individual Differences, 10, 535-540. doi:10.1016/0191-8869(89)90035-4

Gudjonsson, G. H., Kopelman, M. D., \& MacKeith, J. A. (1999). Unreliable admissions to homicide. A case of misdiagnosis of amnesia and misuse of abreaction technique. The British Journal of Psychiatry, 174, 455-459. doi:10.1192/bjp.174.5.455

Gudjonsson, G. H., \& Sigurdsson, J. F. (2004). The relationship of suggestibility and compliance with self-deception and other-deception. Psychology, Crime \& Law, 10, 447-453. doi:10.1 080/10683160310001634278

Gudjonsson, G. H., Sigurdsson, J. F., Sigurdardottir, A. S., Steinthorsson, H., \& Sigurdardottir, V. M. (2014). The role of memory distrust in cases of internalised false confession. Applied Cognitive Psychology, 28, 336-348. doi:10.1002/acp.3002

Haidt, J. (2001). The emotional dog and its rational tail: A social intuitionist approach to moral judgment. Psychological Review, 108, 814-834. doi:10.1037/0033-295X.108.4.814

Hall, L., Johansson, P., \& Strandberg, T. (2012). Lifting the veil of morality: choice blindness and attitude reversals on a self-transforming survey. PLOS ONE, 7, e45457. doi:10.1371/journal. pone. 0045457

Hall, L., Johansson, P., Tärning, B., Sikström, S., \& Deutgen, T. (2010). Magic at the marketplace: Choice blindness for the taste of jam and the smell of tea. Cognition, 117, 54-61. doi:10.1016/j.cognition.2010.06.010 
Hall, L., Strandberg, T., Pärnamets, P., Lind, A., Tärning, B., \& Johansson, P. (2013). How the polls can be both spot on and dead wrong: Using choice blindness to shift political attitudes and voter intentions. PLOS ONE, 8, e60554. doi:10.1371/journal.pone.0060554

Hancock, K. J., \& Rhodes, G. (2008). Contact, configural coding and the other-race effect in face recognition. British Journal of Psychology, 99, 45-56. doi:10.1348/000712607×199981

Hanley, J. R., \& Damjanovic, L. (2009). It is more difficult to retrieve a familiar person's name and occupation from their voice than from their blurred face. Memory, 17, 830-839. doi:10.1080/09658210903264175

Hanley, S. T., Smith, J., \& Hadfield, J. R. (1998). I recognise you but i can't place you: An investigation of familiar-only experiences during tests of voice and face recognition. Quarterly Journal of Experimental Psychology: Section A, 51, 179-195. doi:10.1080/027249898391819

Hastie, R., Landsman, R., \& Loftus, E. F. (1978). Eyewitness testimony: The dangers of guessing. Jurimetrics Journal, 19, 1-8.

Heath, W. P., \& Erickson, J. R. (1998). Memory for central and peripheral actions and props after varied post-event presentation. Legal and Criminological Psychology, 3, 321-346. doi:10.1111/j.2044-8333.1998.tb00369.x

Hell, W., Gigerenzer, G., Gauggel, S., Mall, M., \& Müller, M. (1988). Hindsight bias: An interaction of automatic and motivational factors? Memory \& Cognition, 16, 533-538. doi:10.3758/ bf03197054

Henkel, L. A., \& Mather, M. (2007). Memory attributions for choices: How beliefs shape our memories. Journal of Memory and Language, 57, 163-176. doi:10.1016/j.jml.2006.08.012

Herman Atkins. (2015). Retrieved from Innocence Project website: http://www.innocenceproject.org/cases-false-imprisonment/herman-atkins?searchterm=herman+at

Higham, P. A. (1998). Believing details known to have been suggested. British Journal of Psychology, 89, 265-283. doi:10.1111/j.2044-8295.1998.tb02684.x

Higham, P. A. (2007). No Special K! A signal detection framework for the strategic regulation of memory accuracy. Journal of Experimental Psychology: General, 136, 1-22. doi:10.1037/0096-3445.136.1.1

Hoffrage, U., Hertwig, R., \& Gigerenzer, G. (2000). Hindsight bias: A by-product of knowledge updating? Journal of Experimental Psychology: Learning, Memory, and Cognition, 26, 566581. doi:10.1037/0278-7393.26.3.566

Horry, R., Memon, A., Wright, D. B., \& Milne, R. (2012). Predictors of eyewitness identification decisions from video lineups in England: A field study. Law and Human Behavior, 36, 257265. doi:10.1037/h0093959

Horry, R., Wright, D. B., \& Tredoux, C. G. (2010). Recognition and context memory for faces from own and other ethnic groups: A remember-know investigation. Memory \& Cognition, 38, 134-141. doi:10.3758/mc.38.2.134 
Hourihan, K. L., Benjamin, A. S., \& Liu, X. (2012). A cross-race effect in metamemory: Predictions of face recognition are more accurate for members of our own race. Journal of Applied Research in Memory and Cognition, 1, 158-162. doi:10.1016/j.jarmac.2012.06.004

Hugenberg, K., Young, S. G., Bernstein, M. J., \& Sacco, D. F. (2010). The categorization-individuation model: An integrative account of the other-race recognition deficit. Psychological Review, 117, 1168-1187. doi:10.1037/a0020463

Isaacowitz, D. M., Toner, K., Goren, D., \& Wilson, H. R. (2008). Looking while unhappy moodcongruent gaze in young adults, positive gaze in older adults. Psychological Science, 19, 848-853. doi:10.1111/j.1467-9280.2008.02167.x

Jacques, P. L. S., \& Levine, B. (2007). Ageing and autobiographical memory for emotional and neutral events. Memory, 15, 129-144. doi:10.1080/09658210601119762

Jenkins, R., White, D., Van Montfort, X., \& Mike Burton, A. (2011). Variability in photos of the same face. Cognition, 121, 313-323. doi:10.1016/j.cognition.2011.08.001

Johansson, P., Hall, L., \& Chater, N. (2012). Preference change through choice. In R. J. Dolan \& T. Sharot (Eds.), Neuroscience of preference and choice: Cognitive and neural mechanisms (pp. 121-141). New York: Academic Press.

Johansson, P., Hall, L., \& Gardenfors, P. (2011). Choice blindness and the non-unitary nature of the human mind. Behavioral and Brain Sciences, 34, 28-29. doi:10.1017/S0140525×10002591

Johansson, P., Hall, L., \& Sikström, S. (2008). From change blindness to choice blindness. Psychologia: An International Journal of Psychology in the Orient, 51, 142-155. doi:10.2117/ psysoc. 2008.142

Johansson, P., Hall, L., Sikström, S., \& Olsson, A. (2005). Failure to detect mismatches between intention and outcome in a simple decision task. Science, 310, 116-119. doi:10.1126/science.1111709

Johansson, P., Hall, L., Sikström, S., Tärning, B., \& Lind, A. (2006). How something can be said about telling more than we can know: On choice blindness and introspection. Consciousness and Cognition, 15, 673-692. doi:10.1016/j.concog.2006.09.004

Johansson, P., Hall, L., Tärning, B., Sikström, S., \& Chater, N. (2013). Choice blindness and preference change: You will like this paper better if you (believe you) chose to read it! Journal of Behavioral Decision Making, Advanced online publication. doi:10.1002/bdm.1807

Johnson, T. P., \& Van de Vijver, F. J. R. (2003). Social desirability in cross-cultural research. In J. A. Harkness, F. J. R. Van de Vijver, P. P. Mohler \& Z. fur Umfragen (Eds.), Cross-cultural survey methods (pp. 195-206). New York John Wiley \& Sons.

Johnston, R. A., \& Edmonds, A. J. (2009). Familiar and unfamiliar face recognition: A review. Memory, 17, 577-596. doi:10.1080/09658210902976969

Kassin, S. M., Dror, I. E., \& Kukucka, J. (2013). The forensic confirmation bias: Problems, perspectives, and proposed solutions. Journal of Applied Research in Memory and Cognition, 2, 42-52. doi:10.1016/j.jarmac.2013.01.001 
Kelley, C. M., \& Sahakyan, L. (2003). Memory, monitoring, and control in the attainment of memory accuracy. Journal of Memory and Language, 48, 704-721. doi:10.1016/S0749596X(02)00504-1

Klassen, D., Hornstra, R. K., \& Anderson, P. B. (1975). Influence of social desirability on symptom and mood reporting in a community survey. Journal of Consulting and Clinical Psychology, 43, 448-452. doi:10.1037/h0076863

Klimesch, W., Schimke, H., \& Pfurtscheller, G. (1993). Alpha frequency, cognitive load and memory performance. Brain Topography, 5, 241-251. doi:10.1007/BF01128991

Kompridis, N. (2000). So we need something else for reason to mean. International Journal of Philosophical Studies, 8, 271-295. doi:10.1080/096725500750039282

Koriat, A., \& Goldsmith, M. (1996). Monitoring and control processes in the strategic regulation of memory accuracy. Psychological Review, 103, 490-517.

Kreiman, J., \& Papcun, G. (1991). Comparing discrimination and recognition of unfamiliar voices. Speech Communication, 10, 265-275. doi:10.1016/0167-6393(91)90016-M

LaBar, K. S., \& Cabeza, R. (2006). Cognitive neuroscience of emotional memory. Nature Reviews Neuroscience, 7, 54-64. doi:10.1038/nrn1825

Leite, W. L., \& Beretvas, S. N. (2005). Validation of scores on the Marlowe-Crowne Social Desirability Scale and the Balanced Inventory of Desirable Responding. Educational and Psychological Measurement, 65, 140-154. doi:10.1177/0013164404267285

Leo, R. A., \& Drizin, S. A. (2010). The three errors: Pathways to false confession and wrongful conviction. In G. D. Lassiter \& C. A. Meissner (Eds.), Police interrogations and false confessions: Current research, practice, and policy recommendations. (pp. 9-30). Washington, DC, US: American Psychological Association.

Levin, D. T. (2000). Race as a visual feature: Using visual search and perceptual discrimination tasks to understand face categories and the cross-race recognition deficit. Journal of Experimental Psychology: General, 129, 559-574. doi:10.1037/0096-3445.129.4.559

Levin, D. T., \& Beale, J. M. (2000). Categorical perception occurs in newly learned faces, otherrace faces, and inverted faces. Perception \& Psychophysics, 62, 386-401. doi:10.3758/ BF03205558

Levin, D. T., Simons, D. J., Angelone, B. L., \& Chabris, C. F. (2002). Memory for centrally attended changing objects in an incidental real-world change detection paradigm. British Journal of Psychology, 93, 289-302. doi:10.1348/000712602760146224

Lieberman, M. D., Ochsner, K. N., Gilbert, D. T., \& Schacter, D. L. (2001). Do amnesics exhibit cognitive dissonance reduction? The role of explicit memory and attention in attitude change. Psychological Science, 12, 135-140. doi:10.1111/1467-9280.00323

Lind, A., Hall, L., Breidegard, B., Balkenius, C., \& Johansson, P. (2014). Speakers' acceptance of real-time speech exchange indicates that we use auditory feedback to specify the meaning of what we say. Psychological Science. doi:10.1177/0956797614529797 
Lindsay, D. S. (1993). Eyewitness suggestibility. Current Directions in Psychological Science, 2, 86-89.

Lindsay, D. S., Johnson, M. K., \& Kwon, P. (1991). Developmental changes in memory source monitoring. Journal of Experimental Child Psychology, 52, 297-318. doi:10.1016/00220965(91)90065-z

Loewenstein, G., \& Small, D. A. (2007). The Scarecrow and the Tin Man: The vicissitudes of human sympathy and caring. Review of General Psychology, 11, 112-126. doi:10.1037/10892680.11.2.112

Loftus, E., \& Ketcham, K. (1991). Ivan the Terrible: John Demjanjuk. Witness for the defense: The accused, the eyewitness, and the expert who puts memory on trial. (pp. 210-242). New York, NY US: St Martin's Press.

Loftus, E. F. (1975). Leading questions and the eyewitness report. Cognitive Psychology, 7, 560572. doi:10.1016/0010-0285(75)90023-7

Loftus, E. F. (1979). Eyewitness testimony. Cambridge, MA: Harvard University Press.

Loftus, E. F. (2005). Planting misinformation in the human mind: A 30-year investigation of the malleability of memory. Learning \& Memory, 12, 361-366. doi:10.1101/Im.94705

Loftus, E. F., \& Hoffman, H. G. (1989). Misinformation and memory: The creation of new memories. Journal of Experimental Psychology: General, 118, 100-104. doi:10.1037/00963445.118.1.100

Loftus, E. F., Miller, D. G., \& Burns, H. J. (1978). Semantic integration of verbal information into a visual memory. Journal of Experimental Psychology: Human Learning and Memory, 4, 19-31. doi:10.1037/0278-7393.4.1.19

Luus, C. A. E., \& Wells, G. L. (1991). Eyewitness identification and the selection of distracters for lineups. Law and Human Behavior, 15, 43-57. doi:10.1007/Bf01044829

Lyyra, P., Mäkelä, H., Hietanen, J. K., \& Astikainen, P. (2014). Implicit binding of facial features during change blindness. PLOS ONE, 9. doi:10.1371/journal.pone.0087682

Malpass, R. S., \& Devine, P. G. (1981). Eyewitness identification: Lineup instructions and the absence of the offender. Journal of Applied Psychology, 66, 482-489. doi:10.1037/00219010.66.4.482

Malsch, M., Haket, V., \& Nijboer, H. (2008). De gevaren van het process-verbaal [The dangers of written eyewitness statements]. Nederlands Juristenblad [Dutch Legal Journal], 41, 25782582 .

Mather, M., \& Johnson, M. K. (2000). Choice-supportive source monitoring: Do our decisions seem better to us as we age? Psychology and Aging, 15, 596-606. doi:10.1037/08827974.15.4.596

Mather, M., Shafir, E., \& Johnson, M. K. (2000a). Misrememberance of options past : Source Monitoring and Choice. Psychological Science, 11, 132-138. doi:10.1111/1467-9280.00228 
Mather, M., Shafir, E., \& Johnson, M. K. (2000b). Misremembrance of options past: Source monitoring and choice. Psychological Science, 11, 132-138. doi:10.1111/1467-9280.00228

Mather, M., Shafir, E., \& Johnson, M. K. (2003). Remembering chosen and assigned options. Memory \& Cognition, 31, 422-433. doi:10.3758/BF03194400

Mathôt, S., Schreij, D., \& Theeuwes, J. (2012). OpenSesame: An open-source, graphical experiment builder for the social sciences. Behavior Research Methods, 44, 314-324. doi:10.3758/ s13428-011-0168-7

Mazzoni, G., \& Vannucci, M. (2007). Hindsight bias, the misinformation effect, and false autobiographical memories. Social Cognition, 25, 203-220. doi:10.1521/soco.2007.25.1.203

McCloskey, M., \& Zaragoza, M. (1985a). Misleading postevent information and memory for events: Arguments and evidence against memory impairment hypotheses. Journal of Experimental Psychology: General, 114, 1-16. doi:10.1037/0096-3445.114.1.1

McCloskey, M., \& Zaragoza, M. (1985b). Misleading postevent information and memory for events: Arguments and evidence against memory impairment hypotheses. Journal of Experimental Psychology: General, 114, 1-16. doi:10.1037/0096-3445.114.1.1

Meissner, C. A., \& Brigham, J. C. (2001). Thirty years of investigating the own-race bias in memory for faces. A meta-analytic review. Psychology, Public Policy and Law, 7, 3-35. doi:10.1037//1076-8971.7.1.3

Merckelbach, H., Jelicic, M., \& Pieters, M. (2011a). Misinformation increases symptom reporting - a test-retest experiment. Journal of the Royal Society of Medicine Short Reports. doi:10.1258/shorts.2011.011062

Merckelbach, H., Jelicic, M., \& Pieters, M. (2011b). The residual effect of feigning: How intentional faking may evolve into a less conscious form of symptom reporting. Journal of Clinical and Experimental Neuropsychology, 33, 131-139. doi:10.1080/13803395.2010.495055

Merckelbach, H., Wessel, I., \& Horselenberg, R. (1997). The accuracy of autobiographical memory: A replication of Barclay \& Wellman (1986). Behavioural and Cognitive Psychotherapy, 25, 103-111. doi:10.1017/s1352465800018336

Michel, C., Corneille, O., \& Rossion, B. (2007). Race categorization modulates holistic face encoding. Cognitive Science, 31, 911-924. doi:10.1080/03640210701530805

Migueles, M., \& García-Bajos, E. (2007). Selective retrieval and induced forgetting in eyewitness memory. Applied Cognitive Psychology, 21, 1157-1172. doi:10.1002/acp.1323

Moore, J., \& Haggard, P. (2006). Commentary on 'How something can be said about telling more than we can know: On choice blindness and introspection'. Consciousness and Cognition, 15, 693-696. doi:10.1016/j.concog.2006.09.003

Morton, J., Hammersley, R. H., \& Bekerian, D. A. (1985). Headed records: A model for memory and its failures. Cognition, 20, 1-23. doi:10.1016/0010-0277(85)90002-2

Mueser, K. T., Grau, B. W., Sussman, S., \& Rosen, A. J. (1984). You're only as pretty as you feel: Facial expression as a determinant of physical attractiveness. Journal of Personality and Social Psychology, 46, 469-478. doi:10.1037/0022-3514.46.2.469 
Mullennix, J. W., Ross, A., Smith, C., Kuykendall, K., Conard, J., \& Barb, S. (2011). Typicality effects on memory for voice: Implications for earwitness testimony. Applied Cognitive Psychology, 25, 29-34. doi:10.1002/acp.1635

Münsterberg, H. (1908). On the witness stand: Essays on psychology and crime. New York: McClure Company.

Myers, J. L., O'Brien, E. J., Balota, D. A., \& Toyofuku, M. L. (1984). Memory search without interference: The role of integration. Cognitive Psychology, 16, 217-242. doi:10.1016/00100285(84)90008-2

Neuschatz, J. S., Preston, E. L., Burkett, A. D., Toglia, M. P., Lampinen, J. M., Neuschatz, J. S., . . . Goodsell, C. A. (2005). The effects of post-identification feedback and age on retrospective eyewitness memory. Applied Cognitive Psychology, 19, 435-453. doi:10.1002/Acp.1084

Nisbett, R. E., \& Wilson, T. D. (1977). Telling more than we can know: Verbal reports on mental processes. Psychological Review, 84, 231-259.

O’Regan, J. K., \& Noë, A. (2001). A sensorimotor account of vision and visual consciousness. Behavioral and Brain Sciences, 24, 939-1031.

Oeberst, A., \& Blank, H. (2012). Undoing suggestive influence on memory: The reversibility of the eyewitness misinformation effect. Cognition, 125, 141-159. doi:10.1016/j.cognition.2012.07.009

Orchard, T. L., \& Yarmey, A. D. (1995). The effects of whispers, voice-sample duration, and voice distinctiveness on criminal speaker identification. Applied Cognitive Psychology, 9, 249260. doi:10.1002/acp.2350090306

Palmer, M. A., Brewer, N., \& Weber, N. (2010). Postidentification feedback affects subsequent eyewitness identification performance. Journal of Experimental Psychology: Applied, 16, 387-398. doi:10.1037/a0021034

Panayiotou, G., \& Vrana, S. R. (2004). The role of self-focus, task difficulty, task self-relevance, and evaluation anxiety in reaction time performance. Motivation and Emotion, 28, 171196. doi:10.1023/B:MOEM.0000032313.69675.0d

Pauker, K. B., Rule, N., \& Ambady, N. (2010). Ambiguity and social perception. In E. Balcetis \& G. D. Lassiter (Eds.), Social psychology of visual perception (pp. 7-26). New York, NY: Psychology Press.

Payne, D. G., Toglia, M. P., \& Anastasi, J. S. (1994). Recognition performance level and the magnitude of the misinformation effect in eyewitness memory. Psychonomic Bulletin \& Review, 1, 376-382. doi:10.3758/bf03213978

Pearse, J., \& Gudjonsson, G. H. (1999). Measuring influential police interviewing tactics: A factor analytic approach. Legal and Criminological Psychology, 4, 221-238. doi:10.1348/135532599167860

Peduzzi, P., Concato, J., Kemper, E., Holford, T. R., \& Feinstein, A. R. (1996). A simulation study of the number of events per variable in logistic regression analysis. Journal of Clinical Epidemiology, 49, 1373-1379. 
Perfect, T. J., \& Weber, N. (2012). How should witnesses regulate the accuracy of their identification decisions: One step forward, two steps back? Journal of Experimental Psychology: Learning, Memory, and Cognition, 38, 1810-1818. doi:10.1037/a0028461

Pham, J. C., Aswani, M. S., Rosen, M., Lee, H., Huddle, M., Weeks, K., \& Pronovost, P. J. (2012). Reducing medical errors and adverse events. Annual Review of Medicine, 63, 447-463.

Phillips, M. R., McAuliff, B. D., Kovera, M. B., \& Cutler, B. L. (1999). Double-blind photoarray administration as a safeguard against investigator bias. Journal of Applied Psychology, 84, 940-951. doi:10.1037/0021-9010.84.6.940

Pohl, R. F. (2007). Ways to assess hindsight bias. Social Cognition, 25, 14-31.

Read, J. D., \& Connolly, D. A. (2007). The effects of delay on long-term memory for witnessed events. In M. P. Toglia, D. F. Ross \& R. C. L. Lindsay (Eds.), The handbook of eyewitness psychology, Vol I: Memory for events. (pp. 117-155). Mahwah, NJ US: Lawrence Erlbaum Associates Publishers.

Rhodes, G., Brake, S., Taylor, K., \& Tan, S. (1989). Expertise and configural coding in face recognition. British Journal of Psychology, 80, 313-331. doi:10.1111/j.2044-8295.1989.tb02323.x

Rhodes, G., Ewing, L., Hayward, W. G., Maurer, D., Mondloch, C. J., \& Tanaka, J. W. (2009). Contact and other-race effects in configural and component processing of faces. British Journal of Psychology, 100, 717-728. doi:10.1348/000712608×396503

Ridderinkhof, K. R., Ullsperger, M., Crone, E. A., \& Nieuwenhuis, S. (2004). The role of the medial frontal cortex in cognitive control. Science 306, 443-447. doi:10.1126/science.1100301

Ridolfi, K., \& Possley, M. (2010). Preventable error: A report on prosecutorial misconduct in California 1997-2009. Santa Clara, CA: Northern California Innocence Project.

Rodin, M. J. (1987). Who is memorable to whom: A study of cognitive disregard. Social Cognition, 5, 144-165. doi:10.1521/soco.1987.5.2.144

Rodriguez, D. N., \& Berry, M. A. (2014). The effect of line-up administrator blindness on the recording of eyewitness identification decisions. Legal and Criminological Psychology, 19, 69-79. doi:10.1111/j.2044-8333.2012.02058.x

Rogers, T. B., Kuiper, N. A., \& Kirker, W. S. (1977). Self-reference and the encoding of personal information. Journal of Personality and Social Psychology, 35, 677-688. doi:10.1037/00223514.35.9.677

Rolls, E. T., Grabenhorst, F., \& Deco, G. (2010). Choice, difficulty, and confidence in the brain. Neurolmage, 53, 694-706. doi:10.1016/j.neuroimage.2010.06.073

Rosenthal, R. (2002). Covert communication in classrooms, clinics, courtrooms, and cubicles. American Psychologist, 57, 839-849. doi:10.1037/0003-066X.57.11.839

Sagana, A., Sauerland, M., \& Merckelbach, H. (2013). Witnesses' blindness for their own facial recognition decisions: A field study. Behavioral Sciences \& the Law, 31, 624-636. doi:10.1002/bsl.2082 
Sagana, A., Sauerland, M., \& Merckelbach, H. (2014a). Memory impairment is not sufficient for choice blindness to occur. Frontiers in Psychology, 5, 449. doi:10.3389/fpsyg.2014.00449

Sagana, A., Sauerland, M., \& Merckelbach, H. (2014b). 'This is the person you selected': Eyewitnesses' blindness for their own facial recognition decisions. Applied Cognitive Psychology, 28, 753-764. doi:10.1002/acp.3062

Sagana, A., Sauerland, M., \& Merckelbach, H. (2015). The effect of choice reversals on blindness for identification decisions. Manuscript submitted for publication.

Saks, M. J., \& Koehler, J. J. (2005). The coming paradigm shift in forensic identification science. Science, 309, 892-895. doi:10.1126/science.1111565

Salti, M., El Karoui, I., Maillet, M., \& Naccache, L. (2014). Cognitive dissonance resolution is related to episodic memory. PLOS ONE, 9. doi:10.1371/journal.pone.0108579

Sanna, L. J., \& Schwarz, N. (2003). Debiasing the hindsight bias: The role of accessibility experiences and (mis)attributions. Journal of Experimental Social Psychology, 39, 287-295. doi:10.1016/S0022-1031(02)00528-0

Sarno, J. A., \& Alley, T. R. (1997). Attractiveness and the memorability of faces: Only a matter of distinctiveness? The American Journal of Psychology, 110, 81-92. doi:10.2307/1423702

Sauerland, M., Mehlkopf, S., Krix, A. C., \& Sagana, A. (in press). Deceiving suspects about the content of their alibis: Consequences for truthful and untruthful suspects. Journal of Forensic Practice.

Sauerland, M., Sagana, A., \& Otgaar, H. (2013). Theoretical and legal issues related to choice blindness for voices. Legal and Criminological Psychology, 18, 371-381. doi:10.1111/j.20448333.2012.02049.x

Sauerland, M., Sagana, A., Otgaar, H., \& Broers, N. J. (2014). Self-relevance does not moderate choice blindness in adolescents and children. PLOS ONE, 9, e98563. doi:10.1371/journal. pone.0098563

Sauerland, M., Sagana, A., \& Sporer, S. L. (2012). Postdicting nonchoosers' eyewitness identification accuracy from photographic showups by using confidence and response times. Law and Human Behavior, 36, 394-403. doi:10.1037/h0093926

Sauerland, M., Schell-Leugers, J. M., \& Sagana, A. (2015). Fabrication puts suspects at risk: Blindness to changes in transgression-related statements. Applied Cognitive Psychology, 29, 544-551. doi:10.1002/acp.3133

Sauerland, M., Schell, J., Collaris, J., Reimer, N., Schneider, M., \& Merckelbach, H. (2013). "Yes, I have sometimes stolen bikes": Blindness for norm-violating behaviors and implications for suspect interrogations. Behavioral Sciences and the Law, 31, 239-255. doi:10.1002/ bsl.2063

Sauerland, M., \& Sporer, S. L. (2009). Fast and confident: Postdicting eyewitness identification accuracy in a field study. Journal of Experimental Psychology: Applied, 15, 46-62. doi:10.1037/a0014560 
Saunders, J. (2009). Memory impairment in the weapon focus effect. Memory \& Cognition, 37, 326-335. doi:10.3758/MC.37.3.326

Schiller, N. O., \& Köster, O. (1998). The ability of expert witnesses to identify voices: a comparison between trained and untrained listeners. Forensic Linguistics, 5, 1-9.

Sears, D. O. (1986). College sophomores in the laboratory: Influences of a narrow data base on social psychology's view of human nature. Journal of Personality and Social Psychology, 51, 515-530. doi:10.1037/0022-3514.51.3.515

Shalvi, S., Eldar, O., \& Bereby-Meyer, Y. (2012). Honesty requires time (and lack of justifications). Psychological Science, 23, 1264-1270. doi:10.1177/0956797612443835

Shalvi, S., Gino, F., Barkan, R., \& Ayal, S. (2015). Self-serving justifications doing wrong and feeling moral. Current Directions in Psychological Science, 24, 125-130. doi:10.1177/0963721414553264

Shapiro, P. N., \& Penrod, S. (1986). Meta-analysis of facial identification studies. Psychological Bulletin, 100, 139-156. doi:10.1037/0033-2909.100.2.139

Sharot, T., Martino, B. D., \& Dolan, R. J. (2009). How choice reveals and shapes expected hedonic outcome. The Journal of Neuroscience, 29, 3760-3765. doi:10.1523/jneurosci.4972-08.2009

Sharot, T., Velasquez, C. M., \& Dolan, R. J. (2010). Do decisions shape preference? Evidence from bind choice. Psychological Science, 21, 1231-1235. doi:10.1177/0956797610379235

Shermer, L. O. N., Rose, K. C., \& Hoffman, A. (2011). Perceptions and credibility: Understanding the nuances of eyewitness testimony. Journal of Contemporary Criminal Justice, 27, 183203. doi:10.1177/1043986211405886

Shore, D. I., Burack, J. A., Miller, D., Joseph, S., \& Enns, J. T. (2006). The development of change detection. Developmental Science, 9, 490-497. doi:10.1111/j.1467-7687.2006.00516.x

Sigelman, L., \& Welch, S. (1993). The contact hypothesis revisited: Black-white interaction and positive racial attitudes. Social Forces, 71, 781-795. doi:10.1093/sf/71.3.781

Simons, D. J., \& Levin, D. T. (1998). Failure to detect changes to people during a real-world interaction. Psychonomic Bulletin and Review, 5, 644-649.

Simons, D. J., \& Rensink, R. A. (2005). Change blindness: Past, present, and future. Trends in Cognitive Sciences, 9, 16-20. doi:10.1016/j.tics.2004.11.006

Sloman, S. A., Fernbach, P. M., \& Hagmayer, Y. (2010). Self-deception requires vagueness. Cognition, 115, 268-281. doi:10.1016/j.cognition.2009.12.017

Slovic, P., \& Fischhoff, B. (1977). On the psychology of experimental surprises. Journal of Experimental Psychology: Human Perception and Performance, 3, 544-551. doi:10.1037/00961523.3.4.544

Smalarz, L., \& Wells, G. L. (2015). Contamination of eyewitness self-reports and the mistaken-identification problem. Current Directions in Psychological Science, 24, 120-124. doi:10.1177/0963721414554394 
Smeets, T., Wolf, O. T., Giesbrecht, T., Sijstermans, K., Telgen, S., \& Joels, M. (2009). Stress selectively and lastingly promotes learning of context-related high arousing information. Psychoneuroendocrinology, 34, 1152-1161. doi:10.1016/j.psyneuen.2009.03.001

Sporer, S. L. (2001a). The cross-race effect: Beyond recognition of faces in the laboratory. Psychology, Public Policy, and Law, 7, 170-200. doi:10.1037/1076-8971.7.1.170

Sporer, S. L. (2001b). Recognizing faces of other ethnic groups: An integration of theories. Psychology, Public Policy, and Law, 7, 36-97. doi:10.1037/1076-8971.7.1.36

Sporer, S. L. (2006). The science of eyewitness testimony has come of age. Psychological Science in the Public Interest, 7, i-ii. doi:10.1111/j.1529-1006.2006.00028.x

Sporer, S. L., Penrod, S., Read, D., \& Cutler, B. (1995). Choosing, confidence, and accuracy: A meta-analysis of the confidence-accuracy relation in eyewitness identification studies. Psychological Bulletin, 118, 315-327. doi:10.1037/0033-2909.118.3.315

Steblay, N. K. (1997). Social influence in eyewitness recall: A meta-analytic review of lineup instruction effects. Law and Human Behavior, 21, 283-297. doi:10.1023/A:1024890732059

Steblay, N. K., \& Dysart, J. E. (2011). Seventy-two tests of the sequential lineup superiority effect: A meta-analysis and policy discussion. Psychology, Public Policy, and Law, 17, 99-139. doi:10.1037/a0021650

Steblay, N. K., Wells, G. L., \& Douglass, A. B. (2014). The eyewitness post identification feedback effect 15 years later: Theoretical and policy implications. Psychology, Public Policy, and Law, 20, 1-18. doi:10.1037/law0000001

Stevenage, S. V., Howland, A., \& Tippelt, A. (2011). Interference in eyewitness and earwitness recognition. Applied Cognitive Psychology, 25, 112-118. doi:10.1002/acp.1649

Stöber, J. (1999). Die Soziale-Erwünschtheits-Skala-17 (SES-17): Entwicklung und erste Befunde zu Reliabilität und Validität [The Social Desirability Scale-17 (SDS-17): Development and first results on reliability and validity]. Diagnostica, 45, 173-177. doi:10.1026//00121924.45.4.173

Sui, J., \& Zhu, Y. (2005). Five-year-olds can show the self-reference advantage. International Journal of Behavioral Development, 29, 382-387. doi:10.1080/01650250500172673

Symons, C. S., \& Johnson, B. T. (1997). The self-reference effect in memory: A meta-analysis. Psychological Bulletin, 121, 371-394. doi:10.1037/0033-2909.121.3.371

Tak, P. J. P. (2008). Issues of procedural law. The Dutch criminal justice system (pp. 81-109). Nijmegen; The Netherlands Wolf Legal Publishers.

Talmi, D., \& Moscovitch, M. (2004). Can semantic relatedness explain the enhancement of memory for emotional words? Memory \& Cognition, 32, 742-751. doi:10.3758/BF03195864

Tredoux, C. G. (1998). Statistical inference on measures of lineup fairness. Law and Human Behavior, 22, 217-237. doi:10.1023/A:1025746220886

Tredoux, C. G. (1999). Statistical considerations when determining measures of lineup size and lineup bias. Applied Cognitive Psychology, 13, 9-26. doi:10.1002/(SICI)1099-0720 
Tuckey, M. R., \& Brewer, N. (2003). The influence of schemas, stimulus ambiguity, and interview schedule on eyewitness memory over time. Journal of Experimental Psychology: Applied, 9, 101-118.

Ullsperger, M., \& von Cramon, D. Y. (2004). Neuroimaging of Performance Monitoring: Error Detection and Beyond. Cortex, 40, 593-604. doi:10.1016/S0010-9452(08)70155-2

Underwood, J., \& Pezdek, K. (1998). Memory suggestibility as an example of the sleeper effect. Psychonomic Bulletin \& Review, 5, 449-453. doi:10.3758/BF03208820

Valentine, T. (1991). A unified account of the effects of distinctiveness, inversion, and race in face recognition. The Quarterly Journal of Experimental Psychology Section A: Human Experimental Psychology, 43, 161-204. doi:10.1080/14640749108400966

Valentine, T. (2001). Face-space models of face recognition. In M. J. Wenger \& J. T. Townsend (Eds.), Computational, geometric, and process perspectives on facial cognition: Contexts and challenges (pp. 83-113). Mahwah, NJ: Lawrence Erlbaum.

van Bergen, S., Horselenberg, R., Merckelbach, H., Jelicic, M., \& Beckers, R. (2010). Memory distrust and acceptance of misinformation. Applied Cognitive Psychology, 24, 885-896. doi:10.1002/acp.1595

Vornik, L., Sharman, S., \& Garry, M. (2003). The power of the spoken word: Sociolinguistic cues influence the misinformation effect. Memory, 11, 101-109. doi:10.1080/741938170

Weber, N., \& Brewer, N. (2004). Confidence-accuracy calibration in absolute and relative face recognition judgments. Journal of Experimental Psychology: Applied, 10, 156-172. doi:10.1037/1076-898X.10.3.156

Weber, N., \& Brewer, N. (2006). Positive versus negative face recognition decisions: confidence, accuracy, and response latency. Applied Cognitive Psychology, 20, 17-31. doi:10.1002/ acp.1166

Weber, N., \& Brewer, N. (2008). Eyewitness recall: Regulation of grain size and the role of confidence. Journal of Experimental Psychology: Applied, 14, 50-60. doi:10.1037/1076$898 \times .14 .1 .50$

Weber, N., \& Perfect, T. J. (2012). Improving eyewitness identification accuracy by screening out those who say they don't know. Law and Human Behavior, 36, 28-36. doi:10.1037/ h0093976

Wells, E. C., \& Pozzulo, J. D. (2006). Accuracy of eyewitnesses with a two-culprit crime: Testing a new identification procedure. Psychology, Crime \& Law, 12, 417-427. doi:10.1080/10683160500050666

Wells, G. L. (1978). Applied eyewitness-testimony research: System variables and estimator variables. Journal of Personality and Social Psychology, 36, 1546-1557. doi:10.1037/00223514.36.12.1546

Wells, G. L. (1984). The psychology of lineup identifications. Journal of Applied Social Psychology, 14, 89-103. doi:10.1111/j.1559-1816.1984.tb02223.x 
Wells, G. L., \& Bradfield, A. L. (1998). "Good, you identified the suspect": Feedback to eyewitnesses distorts their reports of the witnessing experience. Journal of Applied Psychology, 83, 360-376. doi:10.1037/0021-9010.83.3.360

Wells, G. L., \& Bradfield, A. L. (1999). Measuring the goodness of lineups: Parameter estimation, question effects, and limits to the mock witness paradigm. Applied Cognitive Psychology, 13, 27-39. doi:0888-4080/99/SIOS27-13

Wells, G. L., \& Luus, C. A. E. (1990). Police lineups as experiments social methodology as a framework for properly conducted lineups. Personality and Social Psychology Bulletin, 16, 106-117. doi:10.1177/0146167290161008

Wells, G. L., Olson, E. A., \& Charman, S. D. (2003). Distorted retrospective eyewitness reports as functions of feedback and delay. Journal of Experimental Psychology: Applied, 9, 42-52. doi:10.1037/1076-898X.9.1.42

Wells, G. L., Small, M., Penrod, S., Malpass, R. S., Fulero, S. M., \& Brimacombe, C. A. E. (1998). Eyewitness identification procedures: Recommendations for lineups and photospreads. Law and Human Behavior, 22, 603-647. doi:10.1023/A:1025750605807

Welte, J. W., \& Russell, M. (1993). Influence of socially desirable responding in a study of stress and substance abuse. Alcoholism: Clinical and Experimental Research, 17, 758-761. doi:10.1111/j.1530-0277.1993.tb00836.x

Wessel, I., \& Merckelbach, H. (1998). Memory for threat-relevant and threat-irrelevant cues in spider phobics. Cognition and Emotion, 12, 93-104. doi:10.1080/026999398379790

Wickham, L. H. V., \& Morris, P. E. (2003). Attractiveness, distinctiveness, and recognition of faces: Attractive faces can be typical or distinctive but are not better recognized. The American Journal of Psychology, 116, 455-468. doi:10.2307/1423503

Williams, P., \& Simons, D. J. (2000). Detecting changes in novel, complex three-dimensional objects. Visual Cognition, 7, 297-322. doi:10.1080/135062800394829

Wixted, J. T., \& Ebbesen, E. B. (1991). On the form of forgetting. Psychological Science, 2, 409415. doi:10.1111/j.1467-9280.1991.tb00175.x

Wixted, J. T., \& Mickes, L. (2014). A signal-detection-based diagnostic-feature-detection model of eyewitness identification. Psychological Review, 121, 262-276. doi:10.1037/a0035940

Wolchover, D. (n.d.). Visual Identification Procedures under PACA Code D Retrieved from http:// www.davidwolchover.co.uk/docs/Visual\%20Identification\%20Procedures.doc

Wright, D. B., Boyd, C. E., \& Tredoux, C. G. (2001). A field study of own-race bias in South Africa and England. Psychology, Public Policy, and Law, 7, 119-133. doi:10.1037/10768971.7.1.119

Wright, D. B., Boyd, C. E., \& Tredoux, C. G. (2003). Inter-racial contact and the own-race bias for face recognition in South Africa and England. Applied Cognitive Psychology, 17, 365-373. doi:10.1002/acp.898 
Wright, D. B., \& Stroud, J. N. (1998). Memory quality and misinformation for peripheral and central objects. Legal and Criminological Psychology, 3, 273-286. doi:10.1111/j.2044-8333.1998. tb00366.x

Yeung, N., \& Summerfield, C. (2012). Metacognition in human decision-making: Confidence and error monitoring. Philosophical Transactions of the Royal Society: Biological Sciences, 367, 1310-1321. doi:10.1098/rstb.2011.0416

Yonan, C. A., \& Sommers, M. S. (2000). The effects of talker familiarity on spoken word identification in younger and older listeners. Psychology and Aging, 15, 88-99. doi:10.1037/08827974.15.1.88

Young, S. G., Hugenberg, K., Bernstein, M. J., \& Sacco, D. F. (2012). Perception and motivation in face recognition: A critical review of theories of the cross-race effect. Personality and Social Psychology Review, 16, 116-142. doi:10.1177/1088868311418987

Zaragoza, M. S., Belli, R. F., \& Payment, K. E. (2006). Misinformation effects and the suggestibility of eyewitness memory. In M. Garry \& H. Hayne (Eds.), Do justice and let the sky fall: Elizabeth F. Loftus and her contributions to science, law, and academic freedom (pp. 35-63). Hillsdale; NJ: Lawrence Erlbaum Associates.

Zhu, L., Guo, X., Li, J., Zheng, L., Wang, Q., \& Yang, Z. (2012). Hippocampal activity is associated with self-descriptiveness effect in memory, whereas self-reference effect in memory depends on medial prefrontal activity. Hippocampus, 22, 1540-1552. doi:10.1002/hipo.20994 

Valorisation 

In writing an addendum about "knowledge valorisation" a clear definition of the term seems to be in order. That is because assigning value to knowledge could be interpreted differently in different settings. According to the Valorisation Centre of Maastricht University "valorisation is the process of creating value from knowledge, by making this knowledge available and suitable for economic and social exploitation and to translate this knowledge into products, services, processes and new business". A carful reader may notice that the definition in itself at times parallels knowledge to a "product" while at other times parallels knowledge to a "thing". The analogy of knowledge as a "product" suggests that knowledge can be traded on a market with buyers and sellers. Therefore, the value of knowledge is determined on economic basis. The analogy of knowledge as a "thing" however, implies that knowledge can be given not only monetary but also social value (Andriessen, 2005). While the view of knowledge as a product is (arguably) appropriate for certain parts of the technical, medicinal and natural sciences, this approach is hardly applicable for the humanities and social sciences (Andriessen, 2005). Research in the field of social sciences generates more than economic value alone. Thus, for scientific disciplines such as psychology and law we certainly have to take a broad perspective on valorisation.

Regardless of discipline, science contributes to the preservation of our social fabric, culture and democracy. Importantly, science and scientific knowledge has its own intrinsic value, beyond the additive value that comes from valorising in terms of societal growth. According to the philosopher Karl Popper (1902-1994), new insights and theories are considered to be valid for as long as they have not been proven wrong and the advancement of science is based on conjecture and refutation (Popper, 1969; as cited in Jonge \& Louwaars, 2009). However, the increasing focus on valorisation through patents and licenses puts constraints on the attempt to confirm or refute scientific findings, a process vital for the scientific advance (Jonge \& Louwaars, 2009). Therefore, naturally raises the question of why universities are turning their back on academic freedom, where scientific knowledge is valued for what it is. Why universities choose to clash with the traditional scientific values of openness, transparency and the sharing of knowledge? According to Jonge and Louwaars (2009) there are three main reasons: a) funding bodies implement the valorisation policies through inclusion of economic parameters in their contracts, b) universities invest in economic valorisation to generate additional income, and strengthen their position in public-private partnerships, c) there is a "herd mentality": everybody seems to invest in intellectual property so universities do the same to maintain their position at the frontier of science and to maximize their freedom to operate.

Within this adverse business environment universities struggle to maintain their status as research institutes and to be given access to resources which allow them to conduct high quality research, as we do in Maastricht University. Though I consider 
the struggle of the universities in many ways unfair I will make use of the constructive ambiguity of the term valorisation and valorise the current work only to its social impact. As highlighted in the closing of the general discussion (Chapter 10), one should consider one's personal moral compass to determine for him or herself how many cases of innocent people incarcerated as a result of choice blindness manipulations constitute a problem worth attention. Thereby, I abstain from assigning any direct or indirect monetary value to human life and dignity as well as social welfare. Finally, note that since the practical relevance and the innovative nature of this line of work have been stated in detail in various chapters of this dissertation, I will repeat them here only briefly.

\section{Relevance}

The present dissertation aimed to establish the relevance of choice blindness for eyewitness testimony, to explore the moderating factors of the phenomenon, and to unravel the mechanisms underlying choice blindness. Through an in depth investigation we replicated and extended previous work thereby showing that by the time witnesses are questioned in court, a large proportion would fail to realise that they are sitting in front of the wrong defendant or that their statements contain inaccuracies. Clearly, mistaken or deliberate manipulations of even minor details can impede the accurate reconstruction of an event, therefore producing long delays on solving a crime, and possibly incriminate innocent suspects. Additionally, the issues immerging from our line of work raise question about the current understanding of decision making, particularly in the eyewitness context.

\section{Target Groups}

This dissertation aimed primarily to answering the question of whether choice blindness in relevant for eyewitness testimony and explore moderating factors for the phenomenon. Thus, it is relevant for law enforcement agencies, the legal professionals and policy makers. Through the present work we illustrated that mistaken or deliberate manipulations can obstruct justice and possibly incriminate innocent suspects. Furthermore, this dissertation must be of interest to members of the scientific community working in various fields including decision making and memory. Additionally, it targets researchers who are interested in the implementation of the choice blindness paradigm in understanding human decision making in various fields such as political and medical and business sciences. Foremost, the present work targets members of the general public who may find themselves in situations similar to those described in the present dissertation. The present results alert them to the possibility of being unaware to surreptitious changes in the outcome of their decision in their encounters in and outside the legal context. 


\section{Activities and Products}

The issues emerging from our line of work directly appeal to and emphasize the importance of blind lineup administration procedures that leave little room for surreptitious manipulations. Specifically, our line of work underscores the importance of the lineup being administered by an officer who is not involved in the case and does not know who the suspect is. If this is not possible the witness should be instructed that the administrator does not know who the suspect is (Wells et al., 1998). Finally, the findings of Chapter 7 underscore the importance of camera recordings during the interviewing process. This is particularly important in light of findings indicating the relevance of blindness phenomena in the field of interrogations and false confessions (Sauerland, Schell, et al., 2013; Sauerland, Schell-Leugers, \& Sagana, 2015).

\section{Innovation}

The work described in the present dissertation has opened a new chapter in the choice blindness literature. Our lab was the first worldwide to examine choice blindness in the context of eyewitness identification and testimony. Our work along with that of the pioneers of choice blindness has stimulated researchers across the Pont, and specifically at Irvine University, to investigate blindness for identification decision and its relation to the misinformation effect.

\section{Schedule and implementation}

Our early work (Chapters 1-4) has already evoked interest outside the scientific community which has resulted in media appearances (e.g., journal appearances in "Limburger" and the "Volkskrant"). As such some of the results of the present dissertation have been communicated to a broader audience, therefore serving the purpose of informing the public about the hazards of blindness manipulations in the eyewitness setting and beyond. Knowledge valorisation is and will continue to be stimulated by communicating findings of this dissertation within the scientific community through journal publications, conference presentations and organised symposia (e.g., choice blindness symposium at the $1^{\text {st }}$ International Convention of Psychological Science, 2015). 


\section{REFERENCES}

Andriessen, D. G. (2005). Value, valuation, and valorisation. Inspirerend innoveren; meerwarde door kennis. Den Haag, Krie. Available at www. weightlesswealth. com.

De Jonge, B., \& Louwaars, N. (2009). Valorizing science: whose values?. EMBO reports, 10, 535-539. doi: 10.1038/embor.2009.113

Popper, K. R (1969). Conjectures and Refutations: The Growth of Scientific Knowledge. London, UK: Routledge.

Sauerland, M., Schell, J., Collaris, J., Reimer, N., Schneider, M., \& Merckelbach, H. (2013). "Yes, I have sometimes stolen bikes": Blindness for norm-violating behaviors and implications for suspect interrogations. Behavioral Sciences and the Law, 31, 239-255. doi:10.1002/ bsl.2063

Sauerland, M., Schell-Leugers, J. M., \& Sagana, A. (2015). Fabrication puts suspects at risk: Blindness to changes in transgression-related statements. Applied Cognitive Psychology. Advance online publication. doi:10.1002/acp.3133

Wells, G. L., Small, M., Penrod, S., Malpass, R. S., Fulero, S. M., \& Brimacombe, C. A. E. (1998). Eyewitness identification procedures: Recommendations for lineups and photospreads. Law and Human Behavior, 22, 603-647. doi:10.1023/A:1025750605807 


\section{ACKNOWLEDGEMENTS}

In this note I would like to thank all those who supported me and accompanied me during the last five years. You were all an essential part for the completion of my PhD. I will always remember you with a smile! Special thanks to the members of the assessment committee for approving this thesis.

First and foremost, I would like to thank my supervisors, Melanie Sauerland and Harald Merckelbach. Melanie, I still vividly remember the first time we met outside a lecture hall during my master studies. Back then I could not possibly imagine that you would be the key person in accomplishing my dream of completing a PhD. Words are not enough to express my gratitude for believing in me, supporting me, advising me and tolerating me. Your door was always open whenever I needed you. My development as a researcher is greatly thanks to you. It was a great pleasure working with you and I hope that we will continue to work together. Dear Harald, getting to know you and work with you changed entirely my view regarding Professors in the most positive way! Always ready to share your wisdom and learn from the others, enthusiastic and welcoming, open-minded yet critical. I still wonder what you saw in me and decided to give me so generously your support. No matter the reason, I am grateful for your trust. It was a privilege working with you.

Furthermore, I wish to thank all those who made the field studies possible; from the Dean and the standing ethical committee, to the security personnel of Maastricht University. Special thanks to Rense Hoekstra. Rense, I appreciate enormously your assistance and you taking a personal interest in the projects. We would not have managed either of the two field studies without your precious help. Next, I would like to thank the more than 30 bachelor and master students who helped with the data collection and made the implementation of studies possible.

I also would like to thank my colleagues of the Forensic Psychology Section for being such a great and accepting group. To my roommates all these years: thank you for coping with me talking to myself and the objects around me! Lotte and Fritz our days as roomies go back to 2011 . Yet, I still remember the warm feeling in my heart the moment I walked into the office to find that you had decorated it so nicely for my birthday. Jill, Linsey, and Nele, I had such a good time sharing a room with you! Jantine and Lorraine, it was great fun having you as roommates. It was a pity we had to separate. I also would like to thank my great paranymphs, Conny and Elly, for agreeing to support me during the defense.

Last but not least, I would like to thank my family and friends who were by my side all these years. Harry thank you much for the Greek style coffee breaks and for the inspirational exchange of ideas, at a scientific and a societal level. I surely miss

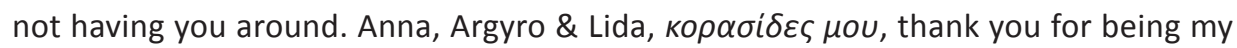


friends despite the years, the distance and my (frequent) communication silences. This very book was the reason!

My dearest Mario, you came to my life during the most frustrating part of this project. Ever since, you transformed not only my life but also my thinking regarding science. Thank you for the long discussions, for your critical comments in parts of this dissertation, for the various proof reads, for always listening and, even more so, for pretending to listen to my absurd monologues. I am very lucky to have you in my life!

My sweet brother, thank you for keeping me in touch with reality; with the world outside the narrow confines of academic life. It is easy to lose track of the important things in life in the "sterilized" world of academia. Thank you for being my life compass!

My beloved parents, I close with my gratitude to you. If it wasn't for you none of this would have been possible. I cannot imagine having more supportive and loving parents than you. The pride in your eyes makes everything worthwhile. However, the effort put into this project is minimal in comparison to your achievement: Your devotion and hard work for over 30 years, without even knowing what the concept of "taking vacations" mean, in order to provide the best for us. I am very proud and thankful for having you as my parents. 


\section{PUBLICATIONS}

\section{Journal Articles}

Sagana, A., Sauerland, M., Merckelbach, H. (in press).The effect of choice reversals on blindness for identification decisions. Psychology, Crime \& Law.

Sauerland, M., Mehlkopf, S., Krix, A., \& Sagana, A. (in press). Deceiving suspects about the content of their alibis: Consequences for truthful and untruthful suspects. Journal of Forensic Practice.

Sauerland, M., Schell-Leugers, J. M., \& Sagana, A. (2015). Fabrication puts suspects at risk: Blindness to changes in transgression-related statements. Applied Cognitive Psychology, 29, 544-551. doi:10.1002/acp.3133

Sagana, A., Sauerland, M., \& Merckelbach, H. (2014). "This is the person you selected": Eyewitnesses' blindness for their own facial recognition decisions. Applied Cognitive Psychology, 28, 753-764, doi:10.1002/acp.3062

Sagana A., Sauerland M. \& Merckelbach H. (2014). Memory impairment is not sufficient for choice blindness to occur. Frontiers in Psychology, 5, doi:10.3389/fpsyg.2014.00449

Sauerland, M., Sagana, A., Otgaar, H., \& Broers, N. J. (2014). Self-relevance does not moderate choice blindness in adolescents and children. Plos One, 9, doi:10.1371/journal. pone. 0098563

Sagana, A., Sauerland, M., \& Merckelbach, H. (2013). Witnesses' blindness for their own facial recognition decisions: A field study. Behavioral Sciences and the Law, 31, 624-636. doi:10.1002/bsl.2082

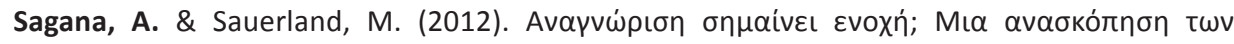

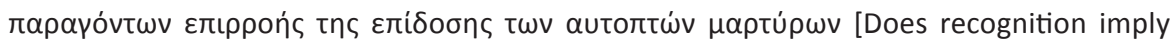
guilt? An overview of the factors that influence eyewitness identification performance]. uvxoגoyia [Psychology], 19, 63-79.

Sagana, A., Sauerland, M., \& Merkelbach, H. (2012). It's your choice! - Or is it really? The Inquisitive Mind, 14/2012. Retrieved from http://beta.in-mind.org/issue-14/it\%E2\%80\%99syour-choice-\%E2\%80\%93-or-it-really

Sauerland, M., Sagana, A., \& Otgaar, H. (2012). Theoretical and legal issues related to choice blindness for voice. Legal and Criminological Psychology, 18, 371-381. doi:10.1111/j.20448333.2012.02049.x

Sauerland, M., Sagana, A., \& Sporer, S. L. (2012). Assessing nonchoosers' eyewitness identification accuracy from photographic showups by using confidence and response times. Law and Human Behavior. 36, 394-403. doi:10.1037/h0093926

\section{Submitted Manuscripts}

Sagana, A., Sauerland, M., \& Merckelbach, H. (2015). Witnesses' failure to detect covert manipulations in their written statements. Manuscript submitted for publication. 
Sauerland, M., Sagana, A., Siegmann, K., Heiligers, D., Merckelbach, H., \& Jenkins, R. (2015). These two are different. Yes, they're the same: Choice blindness for facial identity. Manuscript submitted for publication.

\section{Manuscript in preparation}

Sagana, A., Sauerland, M., Merckelbach, H., \& Broers, N. (2015). Eyewitnesses' blindness for own and other race identification decisions. Manuscript in preparation.

\section{Conference Presentations}

Sagana, A., Cochran, K. J. (2015, March) Raising the blinds: Understanding the underpinnings of choice-blindness across four practical contexts. Symposium organised at the $1^{\text {st }}$ International Convention of Psychological Science in Amsterdam, the Netherlands

Sagana, A., Sauerland, M. (2013, September) The influences of own-race bias on blindness for one's identification decisions. Paper presented at the $22^{\text {nd }}$ meeting of the European Association of Psychology and Law in Coventry, UK.

Sauerland, M., Schell, J. M., \& Sagana, A. (2013, September). How interrogators can make suspects agree to statements they did not make. Paper presented at the 22nd meeting of the European Association of Psychology and Law in Coventry, UK.

Sagana, A., Sauerland, M., Merckelbach, H. (2013, March) Blind identification decisions: The role of personality traits and cognitive factors. Paper presented at the Experimental Psychopathology PhD Candidates Day in Utrecht, The Netherlands

Sagana, A., Sauerland, M., Merckelbach, H. (2012, April). How does choice blindness affect eyewitness identification performance in simultaneous lineups? Paper presented at the $21^{\text {st }}$ meeting of the European Association of Psychology and Law in Nicosia, Cyprus.

Sagana, A., \& Sauerland, M. (2012, March). "This is not who I picked". The choice blindness effect in eyewitness identifications. Paper presented at the annual meeting of the American Psychology-Law Society in San Juan, Puerto Rico.

Sagana, A. \& Sauerland, M. (July 2010). Do foils safeguard against false identifications decisions? Brief oral presentation at the $27^{\text {th }}$ International Congress of Applied Psychology (ICAP), Melbourne, Australia 



\section{ABOUT THE AUTHOR}

Anna Sagana was born on March 22nd, 1986 in Kalamata, Greece. After graduating from secondary school in 2003, she was admitted to the Programme of Psychology at the National and Kapodistrian University of Athens, Greece. During her bachelor studies she served as founding member and president of the Greek Psychology Students' Association, as well as vice president of the European Federation of Psychology Students' Associations. Anna obtained her Bachelor of Science degree in Psychology in December 2008. Subsequently, she joined the Psychology and Law track of the Applied Cognitive Psychology Master at Maastricht University, the Netherlands, from which she graduated in 2009. For her Master thesis, she conducted research examining postdictors of accuracy in eyewitness identification decisions. From 2010 to 2015 she was a doctoral candidate at the Forensic section of Psychology and Neuroscience at Maastricht University. Her research was on the effect of choice blindness on eyewitness testimony. During her doctoral studies, she served as the president of the Student's Division of the International Association of Applied Psychology. Anna currently works as a lecturer at the Faculty of Psychology and Neuroscience of Maastricht University. Her research interests lie in eyewitness identification, choice blindness, memory and decision making.

ISBN : 978-94-6299-169-9 